This work was funded by the US Department of Energy. Office of Energy Research.

The Laboratory's equal opportunity policy requires, in accordance with applicable state and tederal taws. Department of Energy policy, and University of California policy. concerted effort to ensure that the Laboratory does nol discriminate. All policies, procedures, and practices are administered for all employees and applicants regardless oí race, color, national origin, religion, sex, sexual orientation, hanoicap, age, veteran status, medical condition (as defined in Section 12926 of the California Government Code), ancestry. marital status, or citizenship, with the limits imposed by law of University of Califorrila policy. The Laboratory also undertakes affirmative action regarding women, minorities, individuals with handicaps, and covered veteraris. This policy of nondiscrimination applies to recruting, employment, compensation, benefits and services, training, advancement, promotion, transfer, termination, and the availability of both informal and formal complaint.resolution procedures.

Inquiries regarding the Laboratory's equal opportunity and affirmative action policies may be directed to the Affirmative Action/Equal Opportunity Officer, Los Alamos Nationa: Laboratory. Los Alamos, New' Mexico 87545. Telephone (505) 665-2117, (FTS) 855-2117.

\title{
DISCLAIMER
}

This report was piepared as an account of work sponsored by an agency of the United States Government. Neither the United States Government nor any agency thereot, nor any of therr employees. makes any warranty. expressed or implied, or assumes any legal liability or responsibility for the accuracy, completeness, or usefuhess of any information, apparatus, product, or process disclosed, or represents that its use would not infringe privately owned rights. Reterence herein to any specific commercial product. process, or service by trade name. trademark, manufacturer, or otherwise, does not necessarily constitute or imply its endorsement, rer:ommentation, or favoring by the United States Government or any agency thereof. The views and opinions of authors expressed herein do not necessarily state or reflect those of the United States Government or any agency thereot 


$$
\text { LA- } 11970-P R
$$

DE91 006614

\section{Progress at LAMPF}

January-December 1989

\section{MASTER}

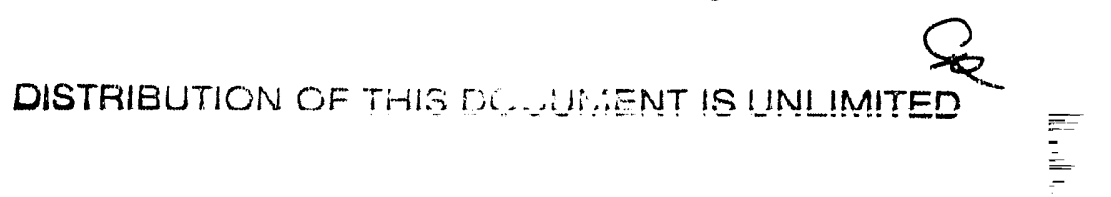




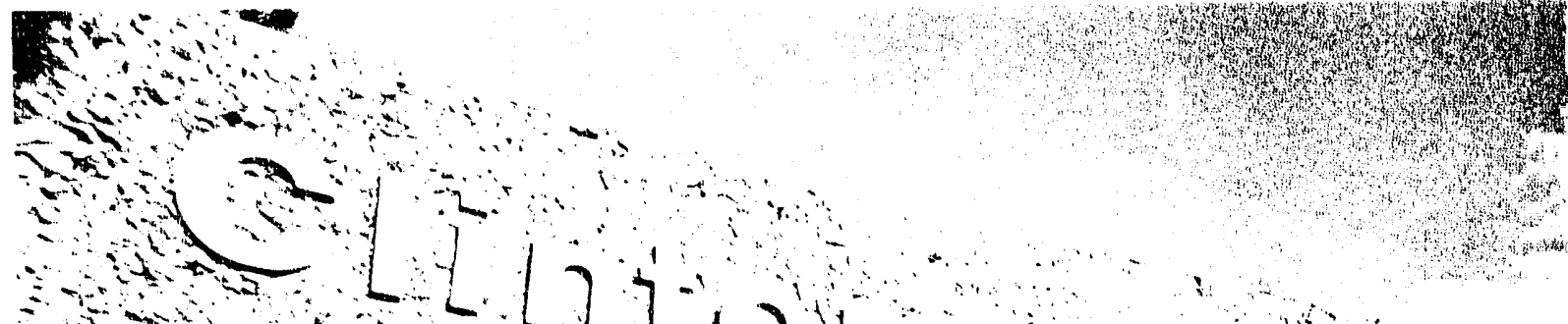

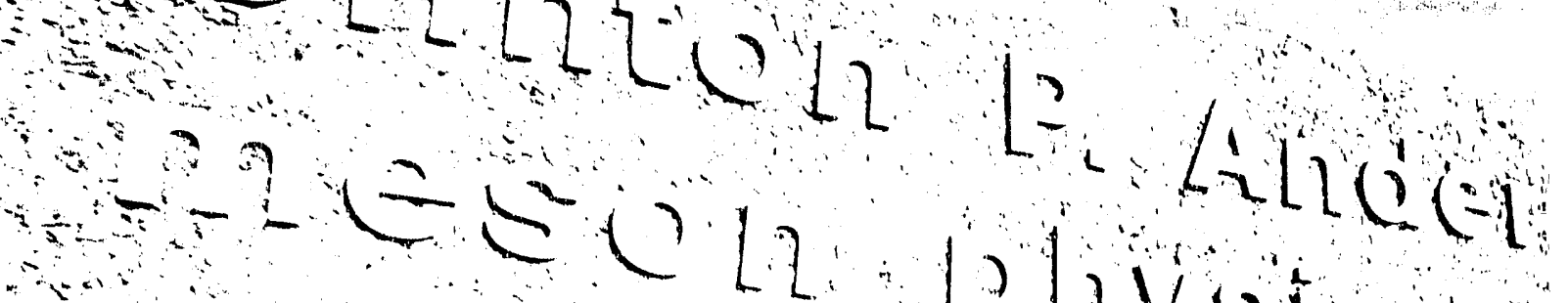

$$
\begin{aligned}
& \text { Llatslos } \\
& \text { HOS ALAKKOS SCHENTHC LH }
\end{aligned}
$$

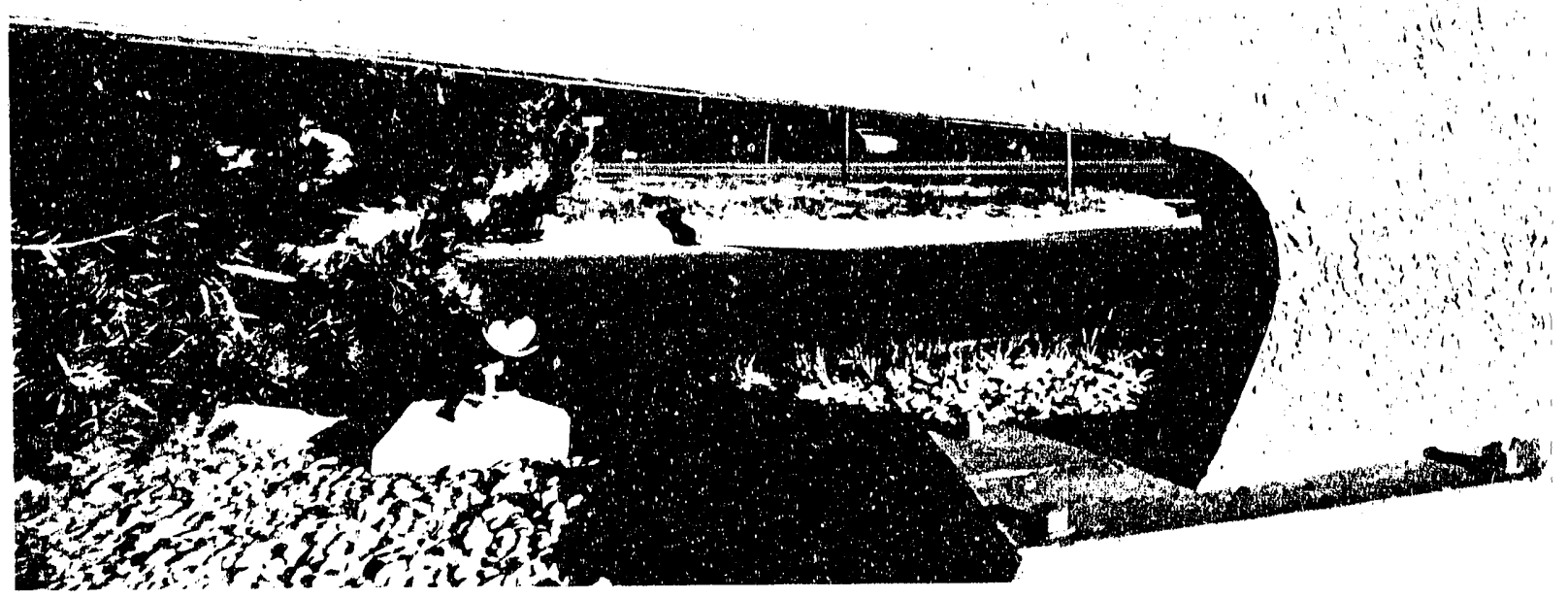




\section{FOREWORD}

We should all take a great deal of pride in how w'ell the Los Alamos Meson Physics Facility (LAMPF) operated during 1989. A new mode of operation was incorporated during the year-multiplexed polarized and unpolarized beam to the external proton beam line (Line X). These two beams, together with the highand low-intensity $\mathrm{H}^{-}$beam for the Proton Storage Ring (PSR) and Weapons Neutron Research (WNR), as well as the high-intensity $\mathrm{H}^{+}$beam to Line A, had LAMPF simultaneously running five separate types of beams to a variety of experimental areas. Congratulations are in order to the accelerator operating staff!

The optically pumped polarized ion source (OPPIS) was commissioned in 1989 and should be incorporated into routine operation in 1990 at $60 \%$ polarization and at least $15 \mu \mathrm{A}$ peak current.

Because of an effective effort throughout MP Division, the PSR has greatly increased both its reliability and peak current. The LAMPF/PSR combination delivered a time-average current of $60 \mu \mathrm{A}$ at $20 \mathrm{~Hz}$ with an overall system reliability of $75 \%$. As the spill time of a PSR pulse is $0.25 / 1 \mathrm{~s}$, the instantaneous current is 12 amperes, which corresponds to an instantaneous power of 10 gigawatts. While the bulk of the neulrons generated by the PSR protons at the Manuel Lujan, Jr. Neutron Scattering Center (LANSCE) is used for research in materials science, a group of nuclear physicists from Triangle Universitics Nuclear Laboratory (TUNL), Los Alamos, Tri-University Meson Facility (TRIUMF), and Delft have carried out a set of pioncering experiments studying parity violation using thermal-epithermal neutrons. The resulting data can be readily analyzed using well-established concepts from the statistical theory of spectra. This approach promises to be a very important development in nuclear physics.

In the area of continuing developments in experimental capability, the use of polarized nuclear targets has become somewhat routine as two definitive mea. surements were carried out involving charge exchange and elastic scattering of pions from polarized ${ }^{13} \mathrm{C}$. The results from these new measurements are presently being analyzed. There are plans for more experiments with polarized ${ }^{13} \mathrm{C}$ and ${ }^{7} \mathrm{Li}$ at the Energetic Pion Channel and Spectrometer (CPICS). The Medium-Resolution Spectrometer (MRS) was commissioned near the end of the last cycle and immediately achieved a resolution of $1.2 \mathrm{MeV}$ at $800 \mathrm{MeV}$, very near the design goal of $1.0 \mathrm{MeV}$. It was also successfully operated in the $(n, p)$ mode in the neutron beam line. Thus, the Nucleon Physics Laboratory (NPL) is ready to go with Neutron Time-of-Flight (NTOF), MRS, and OPPIS. We can look forward to a great increase in the knowledge of the spin and isospin dependence of nuclear structure and reactions from nucleon-nucleus scattering experiments over the next five or so years.

Looking to the future, we plan to put into operation in 1990 a superconducting rf cavity termed the SCRINNCHER on the Low-Energy Pion (LEP) beam line. The cavity is referred to as a SCRUNCHER because it compresses longitudinal phase space of the pion beam in either energy or time to satisfy the requirements of a given experiment. For example, at a fixed momentum resolution, by 
compressing the beam in energy, it can increase the pion flux delivered to an experiment by a factor of 5 .

In conjunction with LAMPF users and other leaders in the nucleur physics community, we are undertaking an examination of future direction for LAMPF in light of the most recent (1989) Nuclear Science Advisory Committee (NSAC) Long-Range Plan for U.S. nuclear physics. Although it appears that a large-scale upgrade to much higher energy at LAMPF is not possible for several years, there are a varicty of enhancements that offer exciting new physics potential. Among those that draw a great deal of support from the LAMPF user community is the development of a bright pion beam at $\sim 10^{9}$ pions/second at $1 \mathrm{GeV}$ to allow high-resolution studies in hypernucleus physics via the $\pi, K$ reaction. There is also strong support among users to further develop the intense, low-duty factor beams from the PSR for muon and neutrino physics. This would be a truly unique source that would alleviate the present requirement at the Line $A$ beam stop source for extensive and expensive active and passive shielding of cosmic rays. Such a development will make neutrino physics appreciably easier and able to address a larger class of problems. There is also a community of nuclear physicists who find the possibility of doing world-class fundamental experiments with cold neutrons in the U.S. an exciting possibility. We are investigating the degree to which LAMPF/PSR/LANSCE can meet this requirement. It also emerged at the NSAC 1989 Long-Range Planning (LRP) meeting that a large segment of the community was interested in carrying out research with beams of exotic short-lived nuclei such as ${ }^{8} \mathrm{He},{ }^{11} \mathrm{Li}$, etc. The most efficient way to produce these nuclei in sufficient quantity is by the use of a $0.6-1 \mathrm{GeV}$ proton beam to initiate spaliation reactions on suitable heavy targets. The shor lived nuclei are then extracted from the target and ionized for acceleration in an appropriate post accelerator. LAMPF has in place the necessary proton beam and infrastructure to b? a natural site for such a facility We are also actively looking into this possibility via a series of workshops and development plans.

Thus, at the present moment, we see many possibilities for the future. However, they all require the necessary support to keep LAMPF a vital laboratory at the forefront of nuclear science. We believe that the Department of Energy wants us to continue in that role and expect that they will provide the resources required to do the job.

Gerald T. Garvey

Director of LAMPF 
LAMPF Users Group ......................................... 1

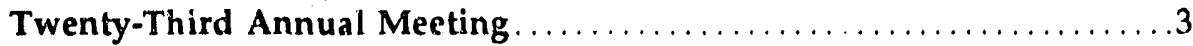

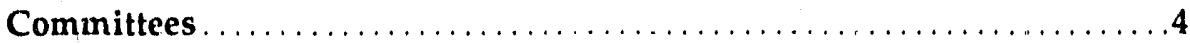

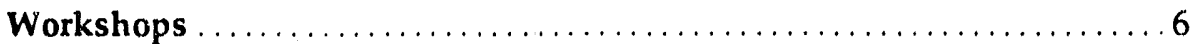

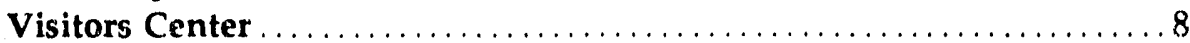

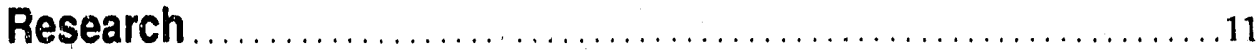

Nuclear and Particle Physics ............................ 13

EXPERIMENT 791 - BNL AGS

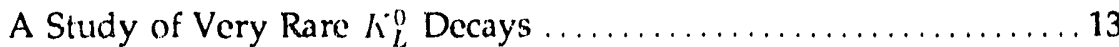

EXPERIMENT 960 - BR

Measurement of $\Delta \sigma_{T}$ and $\Delta \sigma_{L}$ in Free Neutron-Proton

Scattering Between 300 and $800 \mathrm{MeV}$

EXPERIMENT 1035 - BR

Three-Spin Measurements in $p p-p$ at $730 \mathrm{MeV}$

EXPERIMENT 1161 - EPICS

Nuclear Structure Effects in DCX to the DIAS for ${ }^{76,78,80,82} \mathrm{Se} \ldots \ldots .23$

EXPERIMENTS 581 AND 704 - Fermilab

Polarized Proton and Antiproton Experiments at Fermilab

EXPERIMENT 772 -... Fermilab

Study of the Nuclear Antiquark Sea with the Drell-Yan

Process at $800 \mathrm{GeV}$

EXPERIMENT 1075 -.. HIRAB

Interaction of Relativistic $\mathrm{H}^{-}$Ions with Matter

EXPERIMENT $1121 \ldots$ HIRAB

High Excitations and Double Escape in the Negative

Hydrogen Ion

EXPERIMENT 1127 - HIRAB

Multiphoton Detachment of Electrons from the $\mathrm{H}^{-}$!on

EXPERIMENT 811 - LEP

Study of Unnatura! Parity States in Nuclei Using Low-Encrgy Pions 
EXPERIMENT 948 - LEP

The Role of Quasi-Deuterons in Pion Absorption on ${ }^{6} \mathrm{Li}$

EXPERIMENT 975 - LEP

Pion-Nucleus Single Charge Exchange at Threshold Energies.

EXPERIMENT 1023 - LEP

Analyzing Power Measurements for the $\left(\pi^{+}, \pi^{0}\right)$ Reaction on a

Polarized ${ }^{13} \mathrm{C}$ Target

EXPERIMENT 1025 - LEP

Pion Elastic Scattering from Polarized ${ }^{13} \mathrm{C}$

EXPERIMENT 1085 - LEP

Pion Absorption on Deuterium Below $20 \mathrm{MeV}$

66

EXPERIMENT 1098 - LEP/Clamshell

Energy Dependence of Low-Energy Pion Double Charge

Exchange

EXPERIMENT 225 - Neutrino-A

Measurement of the Exclusive Cross Section ${ }^{12} \mathrm{C}\left(\nu_{e}, e^{-}\right)^{12} \mathrm{~N}($ g.s. $) \ldots 71$

EXPERIMENT 225 - Neutrino-A

A Study of Neutrino-Electron Elastic Scattering

EXPERIMENT 645 - Neutrino-A

A Search for Neutrino Oscillations

EXPERIMENT 1173 - Neutrino-A

Search for Neutrino Oscillations with High Sensitivity in the Appearance Channels $\nu_{\mu} \rightarrow \nu_{e}$ and $\bar{\nu}_{\mu} \rightarrow \bar{\nu}_{e}$

EXPERIMENT 1123 - NTOF

The ${ }^{16} \mathrm{O}(p, n){ }^{16} \mathrm{~F}$ Reaction at $500 \mathrm{McV}$

EXPERIMENT $750 \ldots \mathrm{P}^{3}$-East

Reaction Mechanism of Inclusive Pion Double Charge

Exchange in ${ }^{4} \mathrm{He}$

EXPERIMENT 1103 - pi3-East

Inclastic Pion Scattering on ${ }^{17} \mathrm{O}$ Above the $\Delta$ Resonance .96 
EXPERIMENT 1107 - $\mathrm{P}^{3}$-East

Studies of Pion Double Charge Exchange Scattering at

Energies Above the $\Delta$ Resonance

EXPERIMENT 1153 - $p^{3}$-East

Quasifree Pion Charge Exchange at $500 \mathrm{MeV}$.

EXPERIMENT 1015 -. PSR

A Large Cerenkov Detector for Neutrino Physics

EXPERIMENT 1183 - PSR

The Study of Time Reversal and Parity Symmetries Using

Epithermal Neutrons

EXPERIMENT 969 - SMC

MEGA: Search for the Rare Decay $\mu^{+} \rightarrow e^{+} \gamma$

Astrophysics

CYGNUS Project.

Atomic and Molecular Physics

EXPERIMENT 963 - S SMC

First Direct Measurement of $\alpha-\mu$ Sticking in $d t-\mu \mathrm{CF}$

EXPERIMENT 1151 - SMC

Experimental Investigation of Muon-Catalyzed Fusion

Materials Science

EXPERIMENT 1115

Magnetic Ordering in $\left(\mathrm{Y}_{1-x} \operatorname{Pr}_{x} \mathrm{Ba}_{2} \mathrm{Cu}_{3} \mathrm{O}_{7}\right.$ as Evidenced by Muon Spin Relaxation

Nuclear Chemistry

EXPERIMENT 1100 - TOFI

Direct Mass Measurements Using the TOFI Spectrometer:

The Neutron-Rich Isotopes of Chlorine through Iron

Radiation Effects

EXPERIMENT 1014 - Radiation-Effects Facility

Proton, Spallation-Neutron, and Fission Neutron Irradiation of Copper

EXPERIMENT 1139 - Beam Stop Irradiation Facility

Testing of Radiation Resistance of Read-Out Chips for Use in High-Rate Nuclear and Particle Physics Applications 
EXPERIMENT 1165 - Spallation Radiation Effects Facility

Synergetic Load Effects on the Beam Entry Window of a

High-Power Spallation Neutron Source 146

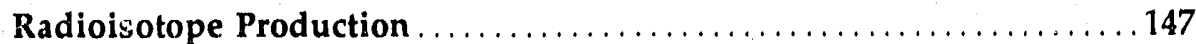

INC-11 Radioisotope Production Activities .................. 147

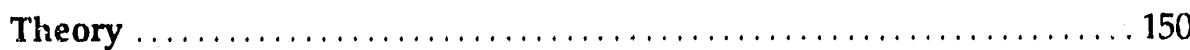

Configuration Mixing as a Source of Three-Body Molecular

Formation it: Muon Catalyzed $d-1$ Fusion .................... 150

Neutron Radius Analysis in the Trinucleon System from

Pion Scattering ................................... 152

Report of the T-5 Theoretical Group .....................172

MP-Division Publications $\ldots \ldots \ldots \ldots \ldots \ldots \ldots \ldots \ldots \ldots \ldots \ldots \ldots$

Facility Development ...................................211

OPPIS: The Optically Pumped Polarized Ion Source $\ldots \ldots \ldots \ldots \ldots, 212$

LAMPF and the Proton Storage Ring (PSR) Control

Systems and Operations .............................. 215

PSR and WNR Beam Delivery Systems ..................... 217

201-MHz RF System Problems ............................. 221

Computing Direntions and Opt ons for Data Acquisition

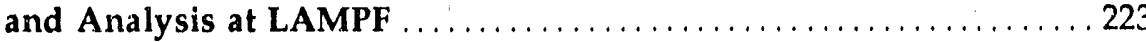

Cost of the LAMPF Linac Extension ........................ 225

Accelerator Operations ................................. .227

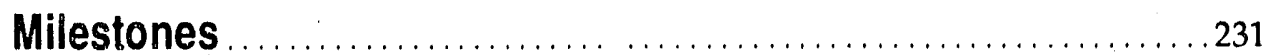

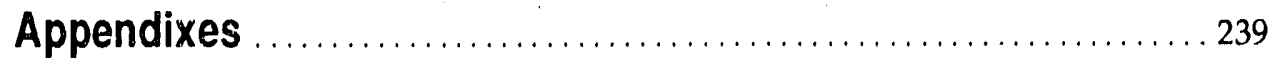

Appendix A:

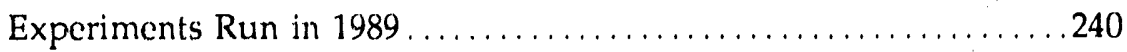

Appendix B:

New Proposals During $1989 \ldots \ldots \ldots \ldots \ldots \ldots \ldots \ldots \ldots \ldots \ldots . \ldots \ldots$

Appendix C:

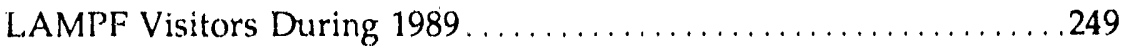

Information for Contributors ...............................259 


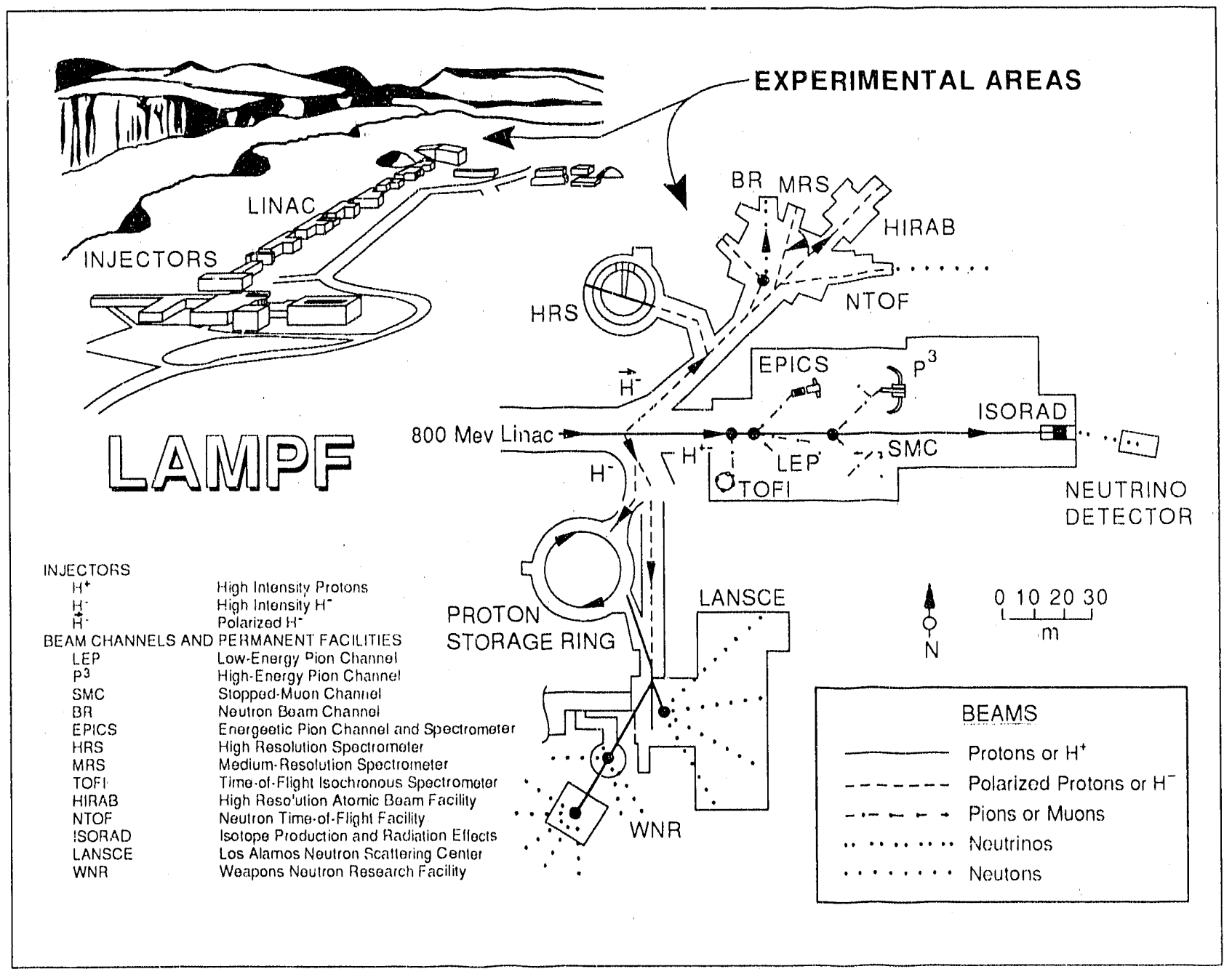




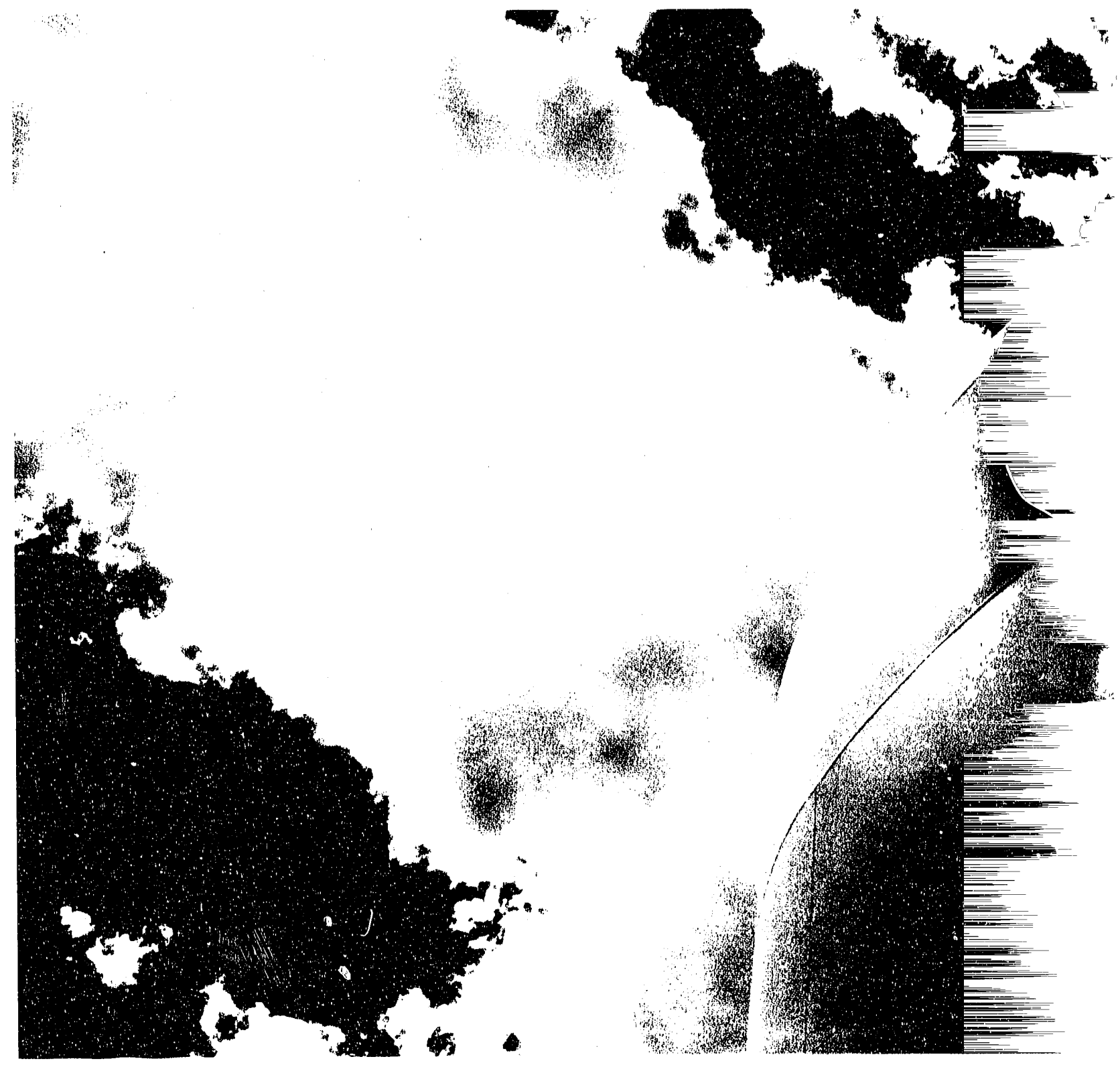




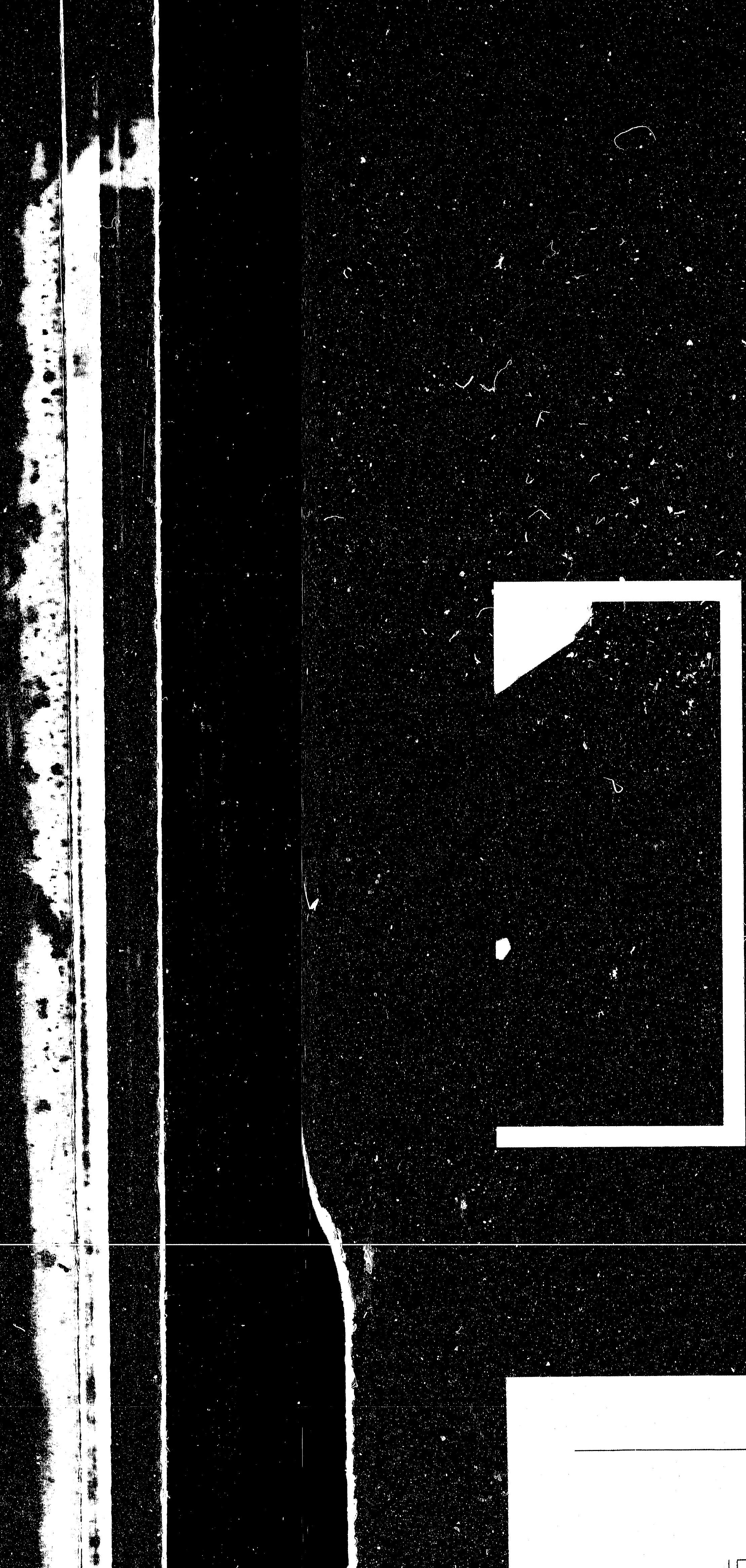


Twenty-Third Annual Meeting
The Twenty-Third Annual Meeting of the LAMPF Users Group, Inc., was held in Los Alamos on August 14-15, 1989, with 134 attendees. Chairman Peter Riley (University of Texas) presided at the first session, which included the following presentations:

"Welcome," Fred Morse, Assuciate Director for Rescarch;

"Report frum Washington," "'ierman Fivozinsky, Department of Encrgy;

"Status of LAMPF," Gerald Garvey, Director of LAMPF;

"Mi'-Division Repor'," Donald Hagerman, MP-Division Leader;

"The Associated Westcrn Universities Program," Thomas Squires, Director (AWU); and

"Report from the Users Group," Peter Riley (University of Texas).

The Louis Rosen Prize was presented to Steirar Hoibraten (Massachusetts Institute of Technology) for his thesis entitled, "Coincidence Measurements of the $\left(\pi^{+}, \pi^{0} p\right)$ Reaction in the $\Delta$-Resonance Region."

The afternoon session was conducted by incoming Chairman Robert McKeown (California Institute of Technology). The following talks were given during the session:

'Future Directions for LAMPF," Gerald Garvey (Los Alamos);

"The Long-Range Planning Process," John McClelland (Los Alamos);

"Accelerator Upgrade Options for LAMPF," Arch Thíessen (Los Alamos);

"Report from the Pion Physics Working Group," George Burleson (New Mexico State University);

"Report from the Electroweak Physics Working Group," Kenneth McFarlane (Temple University); and

"Rep.,rt from the Nucleon Physics Working Group," Peter Riley (University of Texas).

On the second day of the meeting the session was conducted by the new Chairman-Elect Jacobo Rapaport (Ohio University). The following talks were given during the session:

"Spin Measurements with a Polarized ${ }^{13} \mathrm{C}$ Target," Gerald Hoffmann (University of Texas);

"Double Giant Resonances from Pion Double Charge Exchange," Shaul Mordechai (Ben-Gurion University and University of Texas); and

"The LANSCE/WNR Research Program," Paul Lisowski (Los Alamos).

During the remainder of the day, various working groups met. 


\section{Committees}

\section{Board of Directors}

The Board of Directors comprises a Liaison Officer and seven members elected by the LAMPF Users Group, Inc., whose interests they represent and promote. They concern themselves with LAMPF programs, policies, future plans, and especially with how users are treated at LAMPF. The Board also nominates new members for the Prograni Advisory Committee (PAC). Users should address problems and suggestions to individual Board members.

The 1990 membership and term expiration dates are listed below.

James Bradbury (Liaison Officer)

Los Alamos

Terms Expiring in 1990

Peter Riley

(Past Chairman)

University of Texas

Wick Haxton

University of Washington

Ed Hurge iord

Unive'siiy of Houston
Terms Expiring in 1991

Robert McKeown (Chairmar:) California Institute of Technology

Harold Spinka Argonne National Labnratory

Evan Sugarbaker

Ohio State University

Terms Expiring in 1992

Jacobo Rapaport

(Chairman-Elect)

Ohio University

\section{Experimental Facilities Panel}

The Experimental Facilities Panel (EFP) provides technical recommendations to the Board of Directors and LAMPF management about the development of experimental facilities and support activities. The chairman sit the Board of Directors will also act as chairman of the EFP. The EFP consists of not more than fifteen (15) members, each of whom serve for two (2) years, chosen so that approximately half of the panel consists of continuing members each year, and so that the major experimental facilities and beam channels are represented. The duties of the EFP members are to: (1) solicit information from the Users and from LAMPF staff on problems, suggested improvements, and future developments; (2) disseminate such information to the Users; (3) report on User activities, problems, and suggestions at meetings of the EFP; and (4) chair working group meetings at the annual Users Meeting. "ne EFP will meet at least twice a year, and members of the Board of Directors and the Liaison Officer are to be members ex officio.

Members and term expiration dates are listed below.

Terms Expiring in 19)0

Michacl Leitch - LEP

Los Alamos
Michanl McNaughton--NPL

Li ; Alarios 
Bernhard Mecking Member-at-Large CEBAF

R. Jerry Peterson - $\mathrm{P}^{3}$ University of Colorado

Greg Smith-Member-at-Large TRIUMF

Terms Expiring in 1992

F. Todd Baker-HRS

University of Georgia

Byron Dieterle - Ncutrino Facilities

University of New Mexico

Dietrich Dehnhard -.. Polarized Targets

University of Minnesota
Gary Hogan-SMC

Los Alamos

James Knudson-Computer Facilities

Los Alamos

Christopher Morris-EPICS Los Alamos

Paul Reeder-Nuclear Chemistry Batelle Pacific Northwest Laboratory

Walt Somrner-Materials Science Los Alamos

Terry Taddeucci-NTOF Los Alamos 
Workshops
LAMPF workshops and meetings in 1989:

Workshop on Future Options for Data Acquisition and Analysis

January 11-13, 1989

Program Advisory Commitiee (PAC)

January 17-20, 1989

Advanced Hadron Facility (AHF) Accelerator

Design Workshop

February 20-25, 1989

MEGA Collaboration Meeting

(Texas Accelerator Center)

February 22-23, 1989

MEGA Collaboration Meeting

(Stanford, California)

June 20-21, 1989

Workshop on Double Charge Exchange

August 9-11, 1189

LAMPF Users Meeting

August 14-15, 1989

Program Advisory Committee (PAC)

August 15-18, 1989

MEGA Collaboration Meeting

November 6-7, 1989

The following LAMPF workshops are scheduled in 1990:

Program Advisory Committee (PAC)

January 15-19, 1990)

MEGA Collaboration Meeting

(Los Angeles, California)

February 25-27, 1991) 
Conference in Computing in High-Energy Physics (Santa Fe, New Mexico)

April 9-13, 1990

International Conference on Particles and Nuclei

(Cambridge, Massachusetts)

June 25-29, 1990

LAMPF Users Meeting

August 20-21, 1990

Program Advisory Committee (PAC)

August 21-24, 1990 
During this report period, 512 research guests worked on LAMPF-related activities or participated in experiments at LAMPF; of these, 244 were foreign visitors.

\section{LAMPF Users Group Membership}

\section{Membership}

Non-Laboratory

Los Alamos National Laboratory

$\underline{221}$

TOTAL

Institutional Distribution

Membership by Institutions

Los Alamos National Laboratory

National or Government Laboratories

U.S. Universities

Industry

Foreign

Hospitals

Nonaffiliated

TOTAL

\section{Number of Institutions}

National or Government Laboratories

U.S. Universities

Industry

Forcign

Hospitals

Nonaffiliated

\section{Regional Breakdown}

East

Pennsylvania, New Jersey, Delaware, Washington $\Gamma \mathrm{C}$,

Massachusetts, New York, Connecticut, Verm ont,

Rhode Island, New Hampshire, Maine

\section{Midwest}

Ohio, Missouri, Kansas, Indiana, Wisconsin, Michigan, Illinois,

North Dakota, South Dakota, Nebraska, Iowa, Minnesota

\section{South}

Maryland, Virginia, Tennessee, Arkansas, West Virginia,

Kentucky, North Carolina, Alabama, Mississippi, Louisiana,

Georgia, Florida, South Carolina 


\section{Southwest, Mountain}

Montana, Idaho, Utah, Wyoming, Arizona, Colorado,

New Mexico (excluding Los Alamos), Oklahoma, Texas

$14.1 \%$ West

Alaska, Hawaii, Nevada, Washington, Oregon, California

$9.8 \%$

Foreign

Los Alamos National Laboratory 


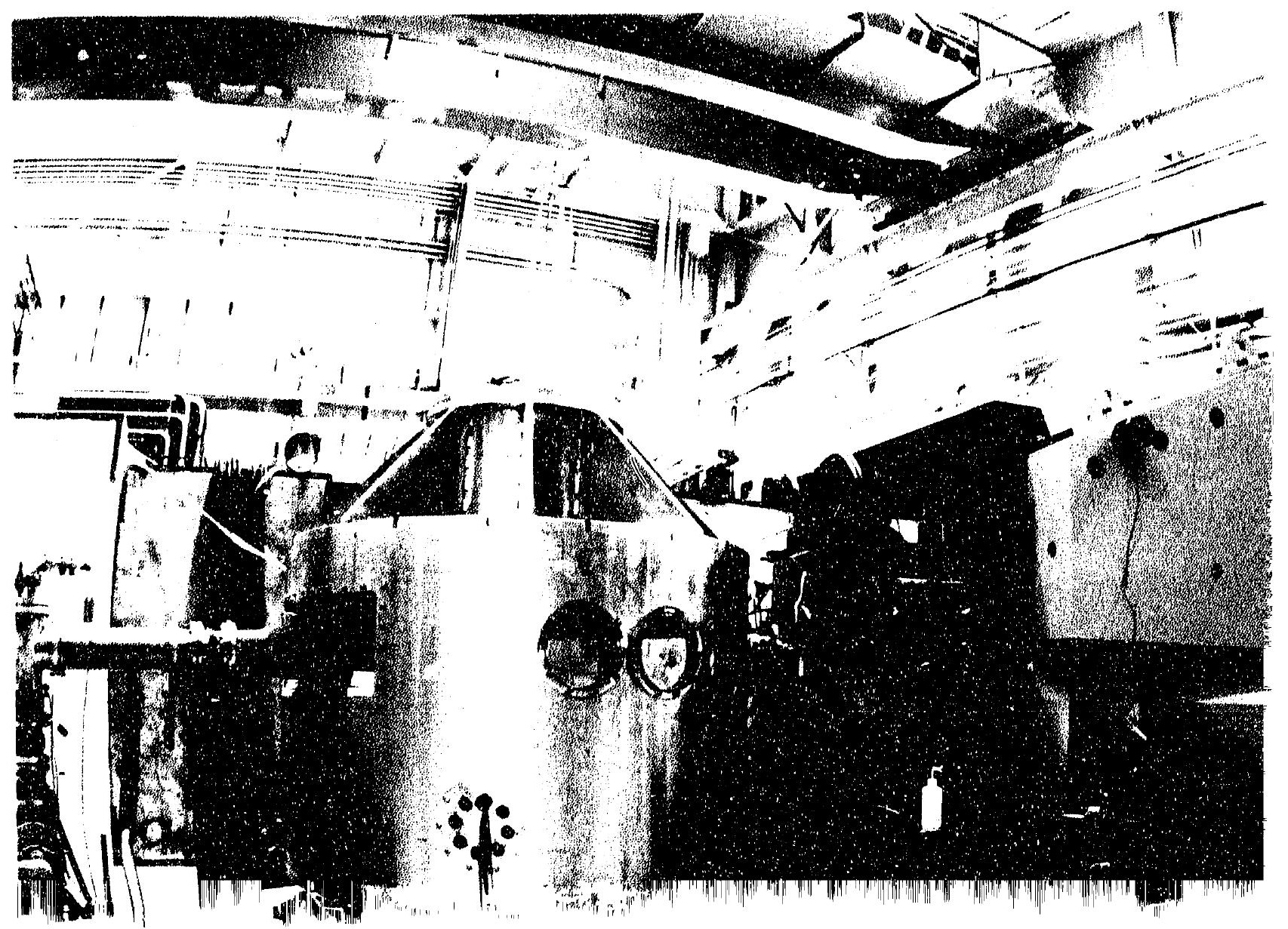




\section{Nuclear and Particle Physics}

EXPERIMENT 791 - BNL AGS

\section{A Study of Very Rare $K_{L}^{0}$ Decays}

UC Irvine, UCLA, Los Alamos, Univ. of Pennsylvania, Staniord Univ., Temple Univ., Univ. of Texas at Austin, College of William \& Mary

Spokesmen: R. D. Cousins (UCLA) and W. R. Molzon (UC Invine)

Participants: A. Heinson, $J$ Horvath, C. Mathiazhagan, W. R. Molzon, K. Arisaka, R. D. Cousins, T. Kaarsberg, J. Konigsberg, J. Kublc, D. Roberts, P. P. Rubin, W. E. Slater, D. Wagner, G. W. Hart, W. W. Kinnison, D. M. Lee, R. J. MaKee, E. C. Milner, G. $H$. Sanders, H. J. Zlock, $P$. Knibbe, J. Urheim, K. A. Blery, M. Dhwan, G. M. Inwin, K. Lang, J. Margulies, D. A. Oulmette, A. Schwartz, S. G. Wojcicki, L. B. Auerbach, J. Belz, P. Buchholz, C. Guss, V. L. Hlghland, S. Ketrell, W. K. McFarlane, M. Siventz, G. W. Hoffmann, J. L. Rilchie, P. Riley, D. Woycheshin, A. Yamashita, M. D. Chapman, M. Eckhause, J. F. Ginkel, A. D. Hancock, J. R. Kane, C. J. Kenney, Y. Kuang, W. F. Vulcan, R. E. Welsh, R. G. Winter, and M. Witkowskl
Experiment 791 at the Brookhaven National Laboratory (BNL) Alternating Gradient Synchrotron is a study of very rare $K_{L}^{0}$ decays, primarily $\kappa_{L}^{0} \rightarrow \mu c$, which is forbidden in the Standard Model of electroweak interactions, and $K_{L}^{\circ}-$, $\mu \mu$ and $K_{L}^{0} \rightarrow c e$, which are allowed. The first decay would violate conservation of separate lepton number, but is an allowed process in many theories seeking to unify the lepton families. While collecting the world's largest sample of $k_{L}^{0} \rightarrow \mu \mu$ events, we continue searching for $K_{L}^{0} \rightarrow c c$. Since the Standard Model prediction $B R\left(K_{L}^{0} \rightarrow e e\right) \approx 3 \times 10^{-12}$ is below our present sensitivity, obscrving this mode would be evidence of new physics.

Discussions of Exp. 791 can be found in papers authored by the collaboration in 1989. Results from the 1988 run were published, ${ }^{1}$ setting $90 \%$-C.L. branching ratio limits of $B\left(K_{L}^{0} \rightarrow \mu e\right)<2.2 \times 10^{-10}$ and $B\left(K_{L}^{\circ} \rightarrow e c\right)<3.2 \times 10^{-10}$. Us sing a set of $87 K_{L}^{0} \rightarrow \mu \mu$ events, we obtained the branching ratio $B\left(K_{L}^{0} \rightarrow \mu \mu\right)=$ $[5.8 \pm 0.6$ (stat) \pm 0.4 (syst) $] \times 10^{-9}$. This is the second report on this experiment in Progress at LAMPF, and the reader is referred to the previous volume ${ }^{2}$ for a discussion of carlier results.

The 1989 data run was 21 weeks long, with an average of $\approx 4.5 \times 10^{12}$ protons per pulse (3.2-s macrocycle time, with a 1.2-s beam spill) striking the production target, yiclding about $1.5 \times 10^{i}$ kaon decays per spill within the spectrometer acceptance. A total of 3500 data tapes were writlen, containing about 90,000 events each. Events from the 2900 physics data tapes in this set were analyzed using the IBM 3090 computers at BNL and Cornell. No examples of the decays, $K_{L}^{0} \rightarrow \mu e$ or $K_{L}^{0} \rightarrow e e$, were found. A preliminary result from this analysis is the identification of about $240 K_{L}^{\circ} \rightarrow \mu \mu$ candidate events. A plot of the reconstructed invariant mass for these events is shown in Fig. 1.

The major obstacle to improving the sensitivity of the experiment for the 1990 run has been the decrease of event paltern recognition efficiency for beam fluxes higher than $4.5 \times 10^{12}$ protons per pulse, caused by the increased singles rate. Changes addressing this issue include:

1. Tightening the "Level 0 " two-body event trigger by improving the resolving time for the scintillator trigger counters from $20 \mathrm{~ns}$ to $14 \mathrm{~ns}$ and for the drift chambers from $56 \mathrm{~ns}$ to $30 \mathrm{~ns}$ during the 1989 run;

2. Installing a new kaon-production target for the 1990 run, having smaller dimensions to define the beam better, and a support structure with less mass near the beam;

3. Installing a new Kevlar window on the vacuum decay region, which allowed removing steel flanges present in the old design;

4. Installing new and larger front drift chambers to improve acceptance; and

5. Using improved pattern recognition algorithms with improved speed and efficiency to identify events in both the on-line selection and offline analysis. 


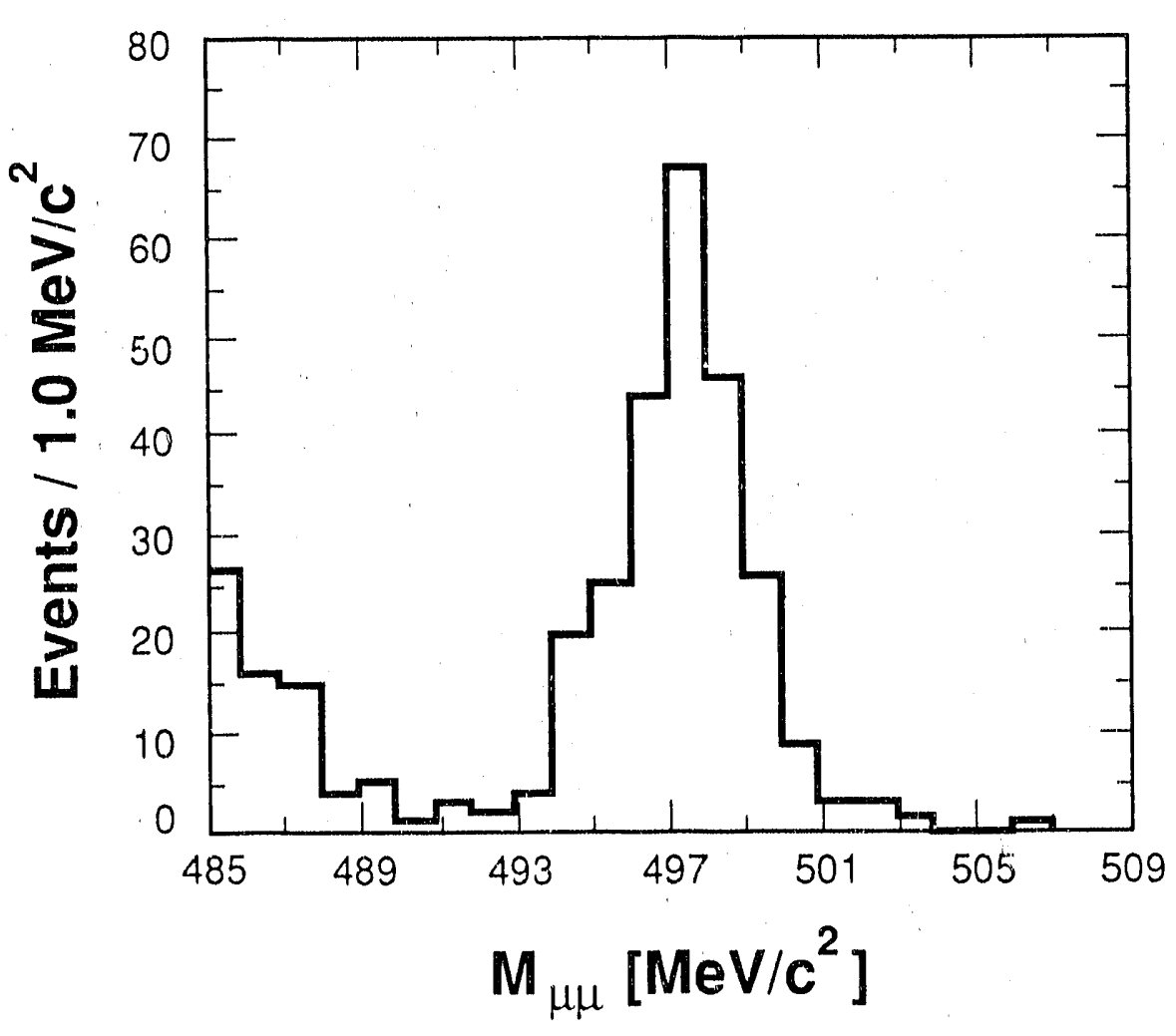

Fig. 1. Plot from the preliminary analysis of the 1989 Exp. 791 data showing the reconstructed invariant mass for the $240 \kappa_{L}^{\circ} \rightarrow \mu \mu$ candidates with collinearity angle less than 1.0 mrad. Collinearity is the angle between the vector from the kaon production target to the decay vertex and the measured momentum vector of the reconstructed decay. Note that the events are well separated from the background contributions appcaring below $488 \mathrm{MeV} / \mathrm{c}^{2}$.

Two experimental collaborations, KEK Exp. 137 and BNL Exp. 791, were conducting searches for $K_{L}^{0} \rightarrow$ e at the beginning of 1989 . The relative sensitivities of these experiments can be judged approximately by the number of $K_{L}^{0} \rightarrow \mu \mu$ events simultancously collected. The Japanese group at Exp. 137 had obtained about $110 \kappa_{L}^{0} \rightarrow \mu \mu$ candidates by November 1989 . By comparison, a total of about $330 K_{L}^{\circ} \rightarrow \mu \mu$ events had been found by the end of 1989 in Exp. 791, and about 300 events may be added to this sample from data collected in 1990. Note that before these experiments began, the world total of $K_{L}^{0} \rightarrow \mu \mu$ events was 28 , gathered in a series of three experiments done about ten years ago. As the present generation of $K_{L}^{0} \rightarrow$ he decay searches nears completion, it appears that the best sensitivity has been obtained in Exp. 791.

To summarize, in Exp. 791 we are leading the search for very rare $k_{L}^{0}$ decays, critically testing the Standard Model. Significant improvement in the limits on 
the decays, $K_{L}^{0} \rightarrow \mu e$ and $K_{L}^{0} \rightarrow e e$, have been obtained, while simultancously collecting the largest number of $K_{L}^{0} \rightarrow \mu \mu$ events ever scen. A four-month long data run commences in early 1990, with the prospect of improving these measurements.

\section{References}

1. C. Mathiazhagan et al., Phys. Rev. Lett. 63, 2181 (1989); C. Mathiazhagan et al., Phys. Rev. Lett. 63, 2185 (1989).

2. "Progress at LAMPF," Los Alamos National Laboratory report LA-11670-PR (1989). 
EXPERIMENT 960 - BR

\section{Measurement of $\Delta \sigma_{T}$ and $\Delta \sigma_{L}$ in Free Neutron-Pro'ion Scattering Between 300 and $800 \mathrm{MeV}$}

\author{
Argonne, Los Alamos, New \\ Mexico State Univ., Texas \\ A\&M Univ., Univ. of \\ Montana, Washington \\ State Univ.
}

Spokesmen: H. Spinka (Argonne), G. Burteson (Now Mexico State Univ.), and L. C. Northclifie (Texas A\&M Unh.)
Experiment 960 is near completion; a first-pass analysis identifying and rejecting bad spills has been completed. Final numbers are expected within the next four months.

The purpose of the experiment, as part of the nucleon-nucleon program of the Argonne Polarized Proton Target (PPT) group, New Mexico State Univcisity, Texas A\&M University, University of Montana, and Washington State University was to measure the difference in the total cross section for a longitudinally polarized beam and target,

$$
\Delta \sigma_{L}(n p)=\frac{\text { yicld }\left(\prod 1\right)-\text { yicld }\left(\prod \uparrow\right)}{\text { yield }(\uparrow 1)+\text { yield }(\uparrow \uparrow)} * \text { target constant },
$$

where the target constant is a term depeiding on the beam, target polarizations, and the target density. The beam, produced from a liquid deuterium target, consisted of polarized neutrons (50\% polarized). The polarized target (cylindrical in shape) was $4.8 \times 12.5 \mathrm{~cm}^{2}$ consisting of propandiol (proton $70 \%$ polarized). The measurement was made using the standard transmission counter techrique.

The energy range selected was between 500 and $800 \mathrm{MeV}$, in approximately $50-\mathrm{MeV}$ steps. The specific energies chosen correspond to previously measured $\Delta \sigma_{L}(p p)$ energies by the Argonne PPT group; ${ }^{1} 497,580,648,733$, and $800 \mathrm{MeV}$. The goal was to measure $\Delta \sigma_{l}(n p)$ with an error of $1 \mathrm{mb}$. Figure 1 shows preliminary values at two energies (shaded boxes), along with previous Argonne data, ${ }^{1}$ and more recent data from Saclay. ${ }^{2}$ Typical errors for these preliminary data are on the order of 1-2 mb; in addition, all the data sets appear consistent (within the $95 \%$ confidence level).

Significant effort has gone into (and continues) understanding systematic effects such as rf-buncher timing stability, beam size and position variations, detector/phototube stability, and intensity (beam) variations. We have found periods where the buncher was unstable and had a significant adverse effect on the measurements. The experiment must be able to correct for systematic asymmetries on the order of $1 \times 10^{-4}$. In addition, there were periods where the operation of Neutron Time-of-Flight (NTOF) had a significant effect on the experiment.

The $n p$ system is an isospin mixture, therefore, it is possible to separate out the isospin singlet $(I=0)$ state through

$$
\Delta \sigma_{L}(I=0)=2 \Delta \sigma_{L}(n p)-\Delta \sigma_{T}(p p) .
$$

Shown in Fig. 2 is $\Delta \sigma_{l}(I=0)$ for these preliminary data, along with the previous Argonne data. The $\Delta \sigma_{l}(p)$ used was from previous Argonne PPT measurements. ${ }^{3}$ There is evidence for structure at $1.5 \mathrm{GcV} / \mathrm{c}$. Several phaseshift analyses have explained the structure as resonances in a variety of partial waves. ${ }^{5}$

Since the reaction under consideration is an isospin singlet $(I=0)$ state and the energy region of interest is below $800 \mathrm{MeV}$, the possible opening of inclastic channels such as $N N-N \Delta$, or $N N \rightarrow N \Delta \pi^{4}$, is not allowed. 


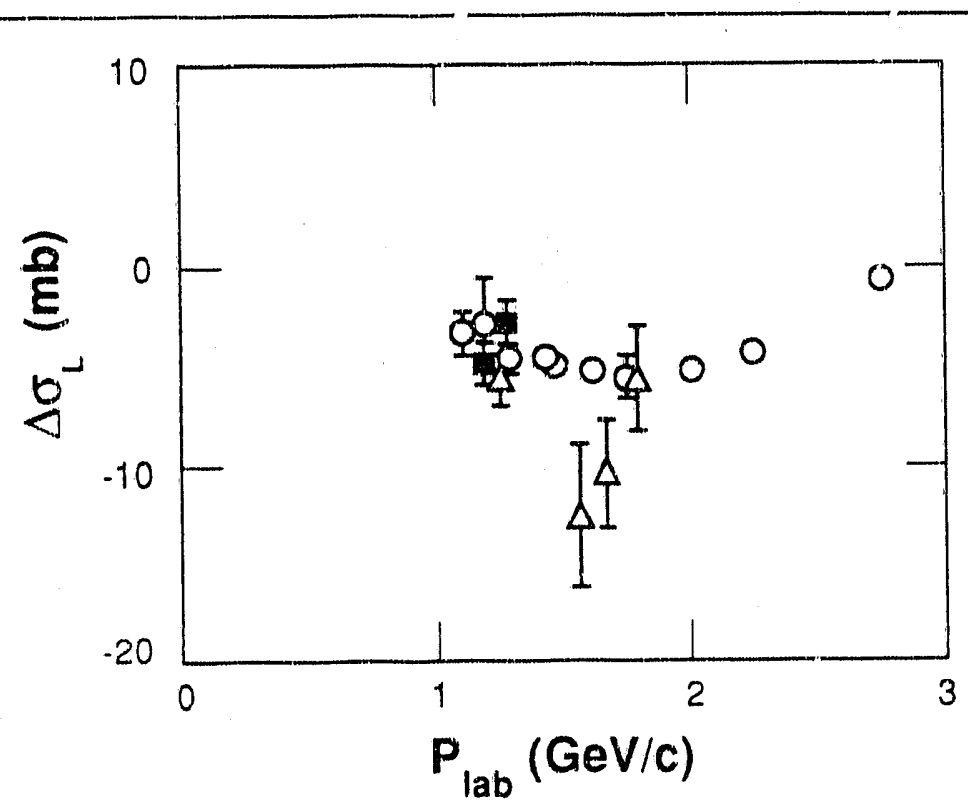

Fig. 1. $\Delta \sigma_{L}$ for the np system. The open boxes represent the Argonne data, the open triangles the Saclay data, and the shaded boxes are our preliminary data.

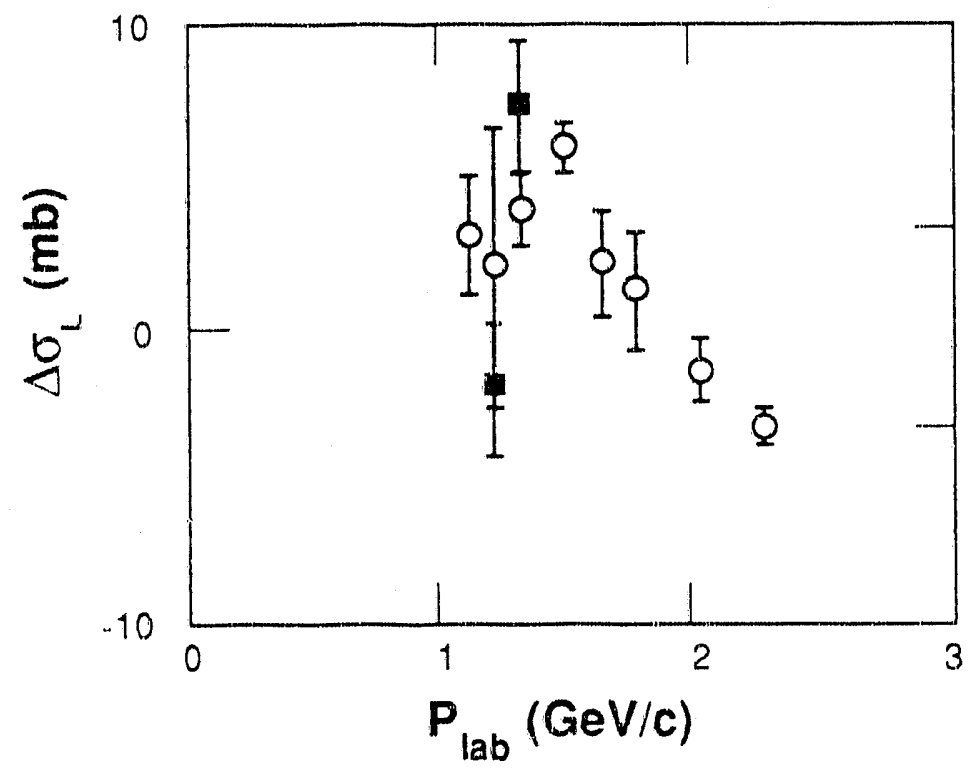

Fig. 2. $\Delta \sigma_{L}$ for the $I=0$ system. The open toxes are the Argonme data and the shaded boxes are our pel!minnryy dota. 


\section{RESEARCH}

Nuclear and Particle Physics

In summary, the preliminary data suggest the following:

1. Past data sets are consistent.

2. It is possible to make Glauber corrections for $p d$ observables $\left(\Delta \sigma_{L}\right)$ to derive the corresponding $p n$ observables.

\section{References}

1. I. P. Auer et al., Phys. Rev. Lett. 46, 1177 (1981); W. Grein, P. Kroll, Nucl. Phys. A 372, 505 (1982).

2. F. Lehar et al., Phys. Lett. B 189, 241 (1987).

3. I. P. Auer et al., Phys. Rev. Lett. 41, 354 (1978).

4. R. L. Shypit et al., Phys. Rev. Lett. 60, 901 (1988).

5. N. Hoshizaki, Phase Shift Analysis of Nucleon-Nucleon Scattering Workshop on the Experiments KEK Polarized Proton and Electron. Beam, October 2728, 1988, KEK National Laboratory, Japan. 
EXPERIMIENT 1035 - BR

\section{Three-Spin Measurements in $p p \rightarrow p p$ at $730 \mathrm{MeV}$}

Los Alamos, Univ. of Texas at Austin, UCLA

Spokesman: M. W. McNaughton (Los Alamos)

Participants: M. W. McNaughton, S. Pentillä, K. H. MeNaughton, P. Riley, E. Gühnez, D. L. Adams, J. Bystricky, and A. Ling

\section{Introduction}

At intermediate energies the $p p$ elastic scattering data set is extensive. The existing data may be examined interactively via Andt's ${ }^{1}$ nucleon-nucleon database and phase-shift fits. But experience has shown that the uncertainties estimated from the phase-shift solutions are too optimistic until the data set is complete enough for a model-independent amplitude analysis. There are five complex $N N$-scattering amplitudes, ${ }^{2}$ so this requires approximately ten independent spin measurements. According to this criterion, the data are complete near $580 \mathrm{MeV}^{3}$ and at 650 and $800 \mathrm{MeV}, 4,5$

We have brought the 730 $\mathrm{MeV}$ data up to this standard by measuring $14 \mathrm{spin}$ observables for $m$ ' scattering (see Fig. 1) and extracting the isospin-1 amplitudes directly from these new data (see Figs. 2 and 3). The observables and amplit ddes are defined by Bystricky and Lehar. ${ }^{2}$

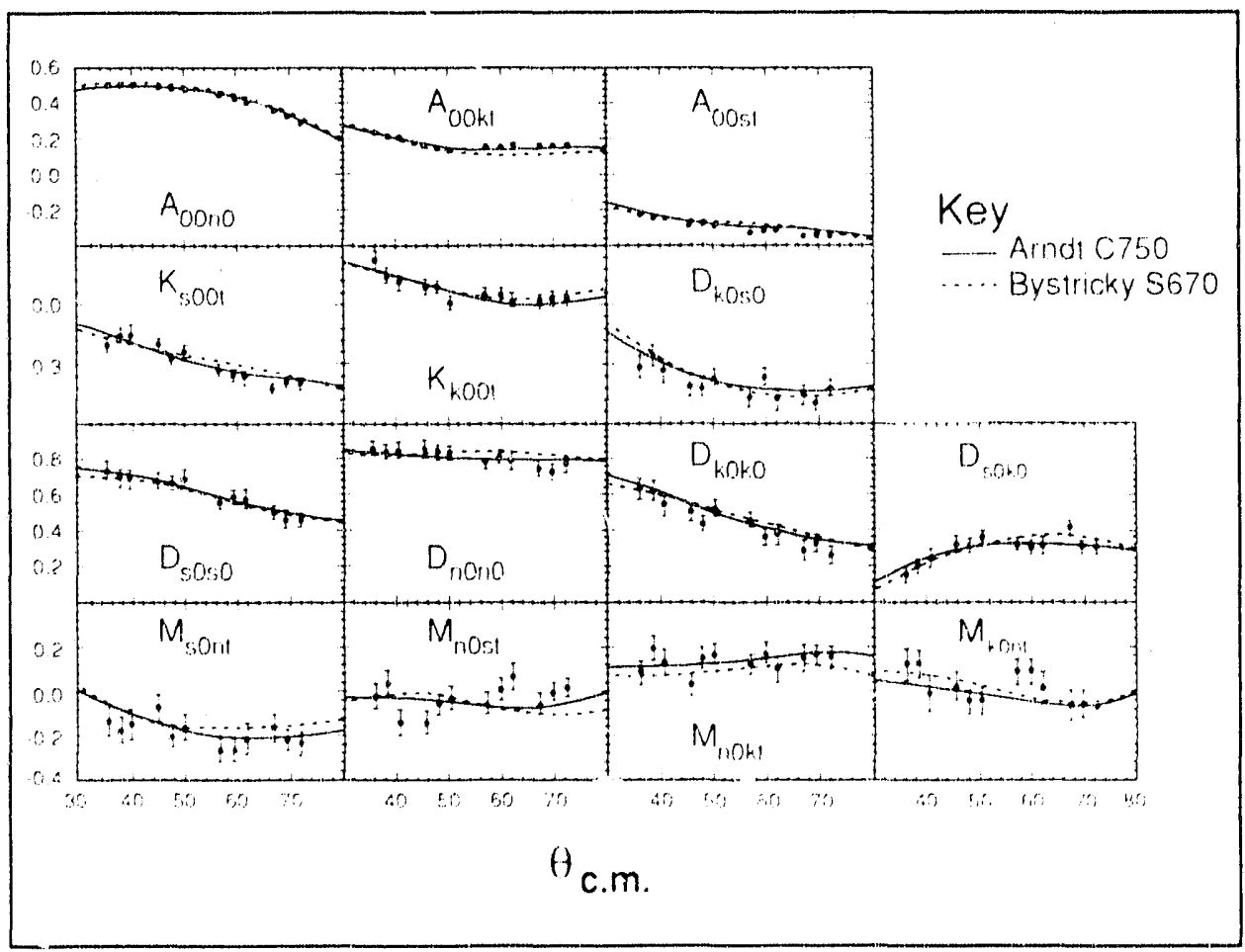

Fig. 1. Spin observables for $p$ relastic scattering at $730 \mathrm{MeV}$ compared with phase-shift solutions by Arndt (C750) and Bystricky (S670).

\section{Experimental Method}

The measurement was made in the external proton beam of LAMPF. The polarized proton beam was directed onto a polarized proton target (PPT) and the polarization of the scattered protons was measured with the Janus ${ }^{6}$ polarimeter, as illustrated in Fig. 4. 


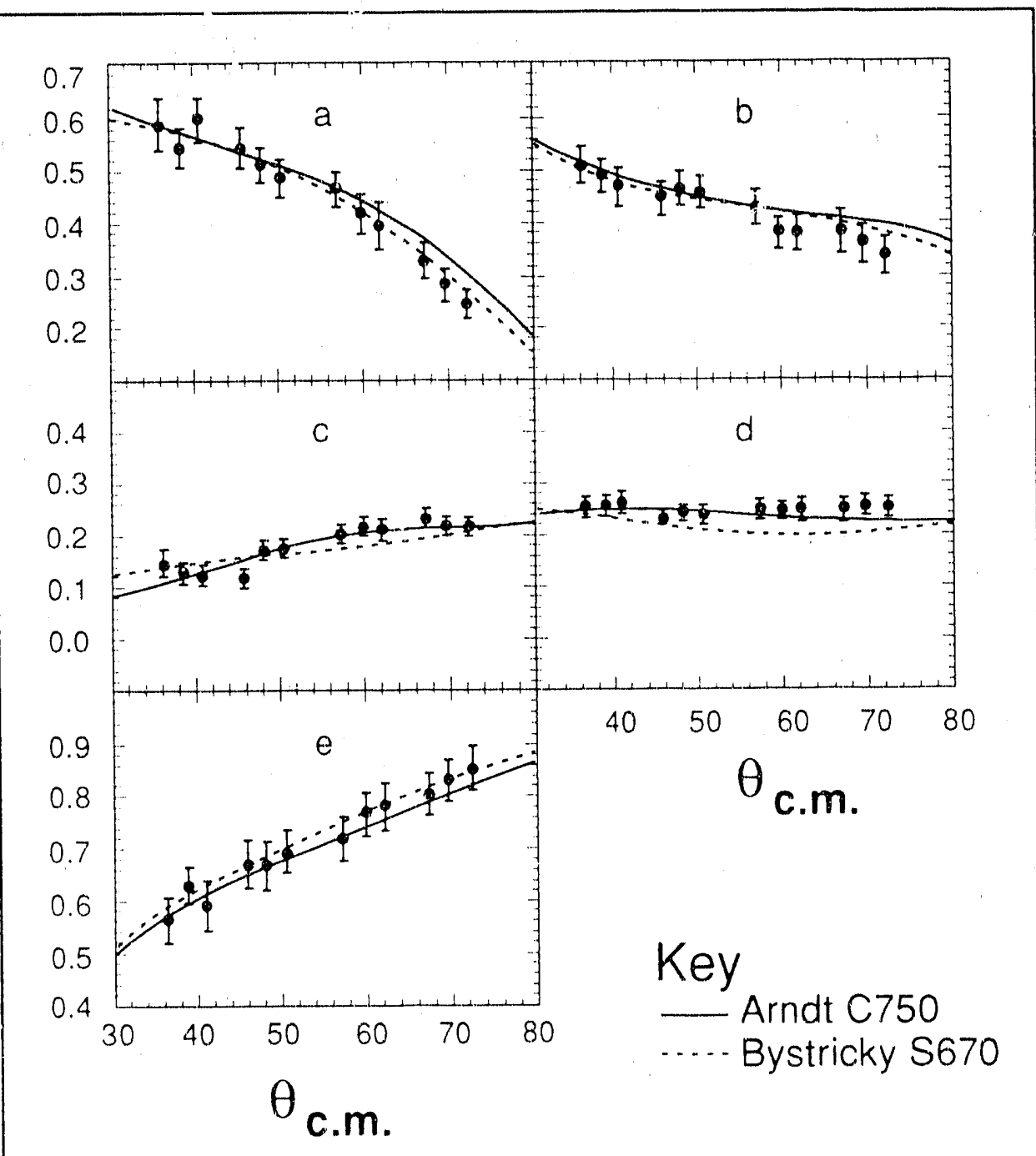

Fig. 2. Magnitudes of the nucleon-nucleon isospin-1 amplitudes; direct reconstructions from the data compared with values from the phase shifts of Arndt (C750) and Bystricky (S670).

The PPT magnet (a superconducting Helmholtz coil) was rotated through an angle $\theta_{t}$ to allow both scrttered and recoil protons to be detected in wire chamber detectors (Fig. 4). Consequently, some of the measured observables are linear combirations of the standard ${ }^{2}$ observables. In principle, we made measurements with all three beam spins $(n, s, k)$, all three scattered proton spins $(n, s, k)$, and the target spin $t$. Therefore, we measured obscrvables of the form $X_{i 0 j t}$, where $i=(n, s, k, 0)$ is the scattered proton spin and $j=(n, s, k, 0)$ is the beam spin. The target spin $t$ is given in terms of the standard unit vectors $\hat{\mathbf{s}}, \hat{\mathbf{n}}, \hat{\mathbf{k}}$ by

$$
\hat{\mathrm{t}} \approx \hat{\mathrm{k}} \cos \theta_{t}+\hat{\mathrm{s}} \sin \theta_{\mathrm{t}}
$$




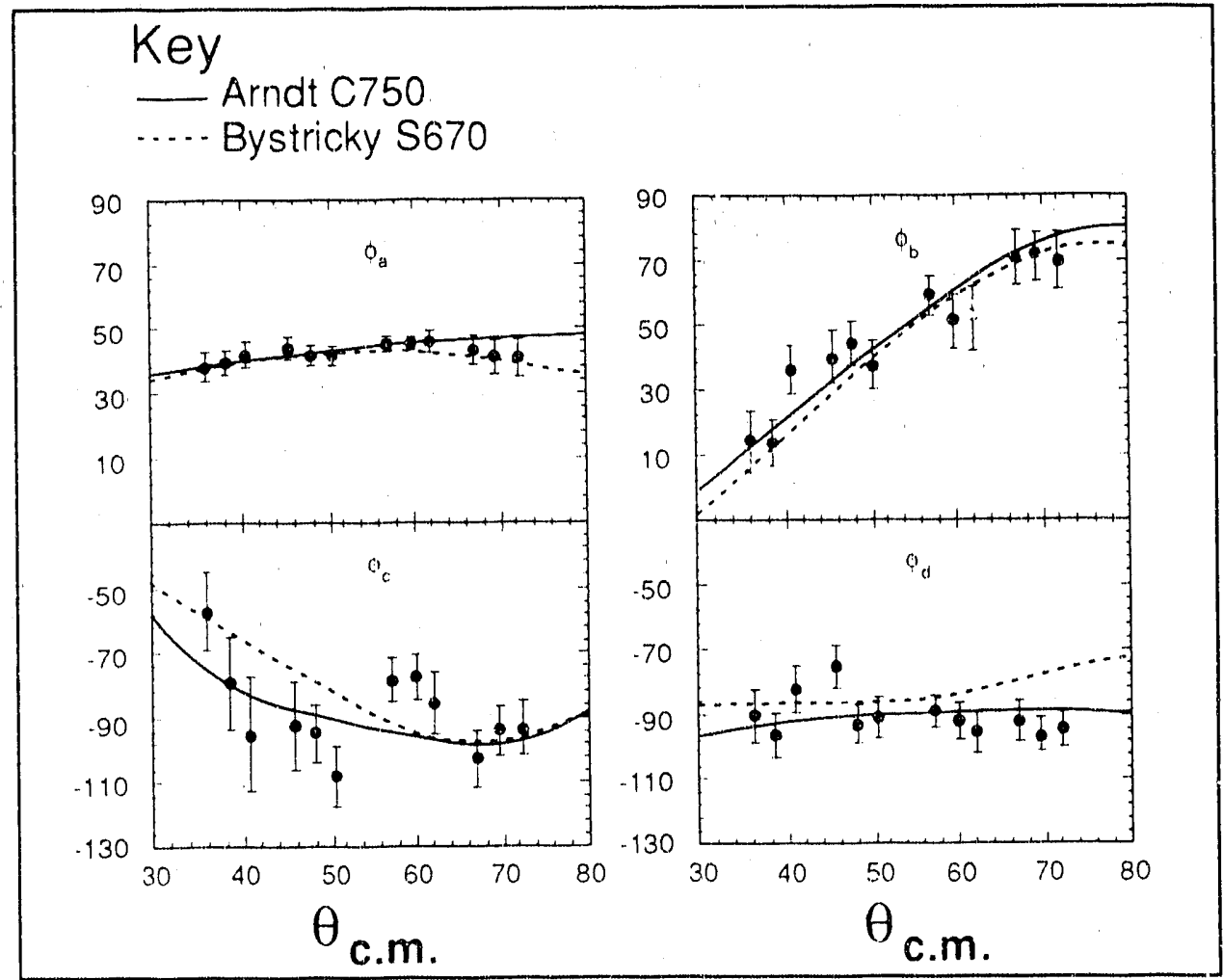

Fig. 3. Phases (relative to $e$ ) of the nucleon-nucleon isospin-1 amplitudes; direct reconstructions from the data compared with values from the phase shifts of Arndt (C750) and Bystricky (5670).

so that the observables are

$$
X_{i 0 j t} \approx X_{i 0 j k} \cos \theta_{t}+X_{i 0 j s} \sin \theta_{t}
$$

\section{Results and Conclusions}

Figure 1 illustrates the data in comparison with the phase-shift fits of Arndt ${ }^{1}$ and Bystricky. ${ }^{\top}$ Agreement is good, which indicates that these new data are consistent with previous data, and that the nucleon-nucleon isospin-1 amplitudes are generally well determined near $730 \mathrm{MeV}$.

The data presented here form a complete set at $730 \mathrm{MeV}$, allowing us to extract the nucleon-nucleon isospin-1 amplitudes directly. The results are shown in comparison with the phase-shift solutions of $\mathrm{Arndt}^{1}$ and Bystricky ${ }^{7}$ in Figs. 2 and 3 .

This completes the measurement of the spin-dependence of the isospin-1 nucleon-nucleon amplitudes from 500 to $800 \mathrm{MeV}$. Completion of the isospin-1 data-set awaits only the publication of the precise absolute cross-section data recently measured at LAMPF (Exp. 1072). 


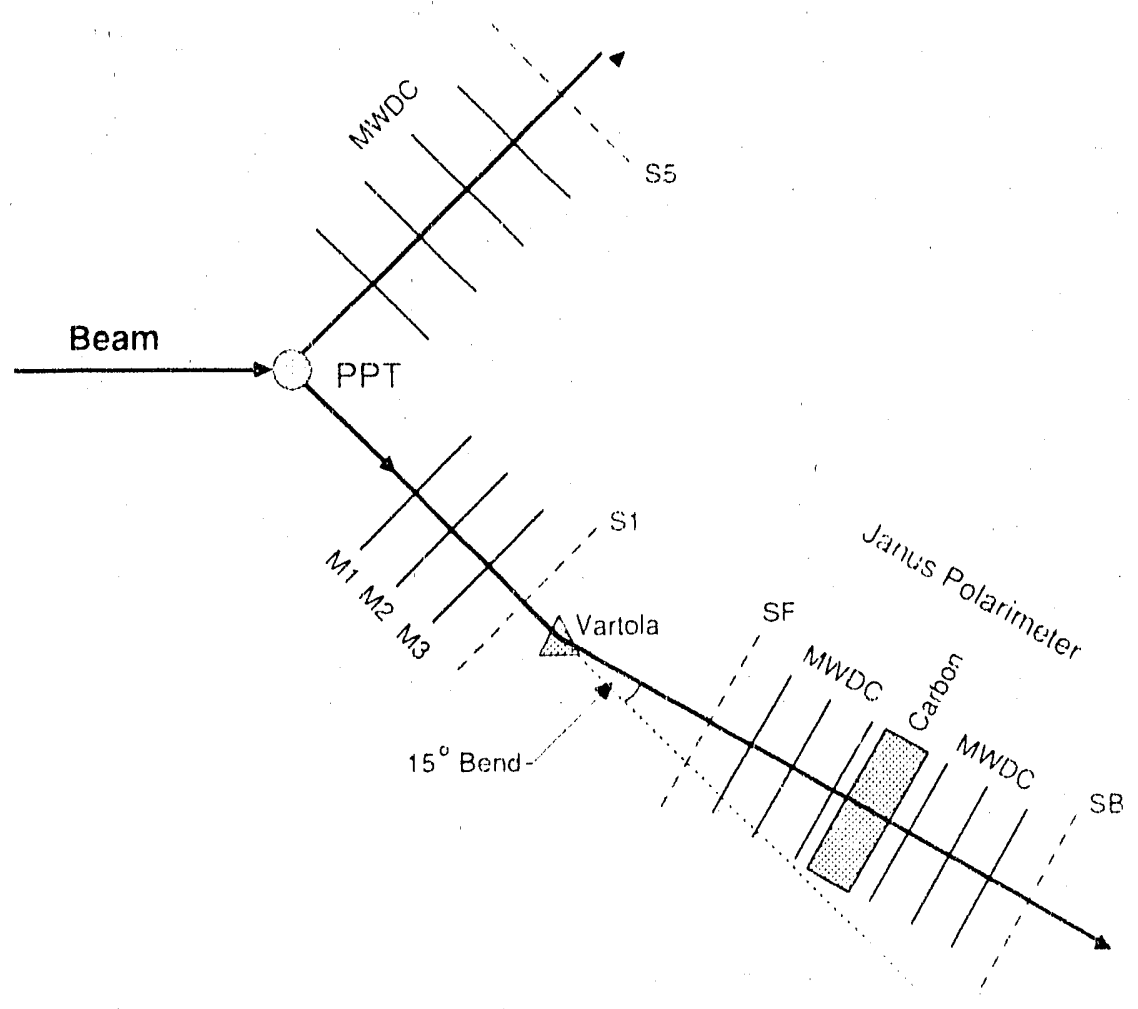

\section{E1035 Experimental Setup}

Fig. 4. The Exp. 1035 apparatus.

\section{References}

1. R. A. Arndt, L. D. Roper, R. A. Bryan, R. B. Clark, B. J. West, P. Signell et al., Phys. Rev. D 28, 97 (1983).

2. J. Bystricky, F. Lehar, and P. Wiritemitz, J. de Physique 39, 1 (1978).

3. R. Hausammann, E. Heer, R. Hess, C. Hechanoine-Leluc, W. R. Leo, Y. Onel et al., Phys. Rev. D 40, 22 (1989).

4. C. L. Hellas, D. J. Cremens, K. H. McNaughton, P. J. Riley, R. F. Rodebaugh, Shen-Wu Xu et al., Phys. Rev. C 30, 1251 (1984).

5. M. J. Moravcsik, F. Arash, and G. R. Goldstein, Phys. Rev. D 31, 1577 (1985).

6. R. Ransome, S. J. Greene, C. L. Hollis, B. E. Bonner, M. W. McNaughton, C. L. Morris et al., Nucl. Instrum. and Methods 201, 309 (1982).

7. J. Bystricky, C. Lechanoine-Leluc, and F. Lehar, J. de Physique 48, 199 (1987). 
EXPERIMENT 1161 - EPICS

\section{Nuclear Structure Effects in DCX to the DIAS for ${ }^{76,78,80,82} \mathrm{Se}$}

Univ. of Pennsylvania, Louisiana State Univ., Ben-Gurion Univ. (Israel), Texas Univ., Rutgers Univ., Univ. of York (Canada), CEBAF

Spokesmen: H. T. Fortune (Unh. of Pennsylvania), A. Fazely (Louisiana State Univ.), and S. Mordechal (Ben-Gurion Univ.)

Participants: R. Gillman, $M$. Kagarlis, J. M. O'Donnell, and D. L. Watson
Data acquisition for a study of the double isobaric analog state (DIAS) at $295 \mathrm{McV}$, on a series of selenium isotopes $(A=76,78,80,82)$, using the EPICS facility was completed this year. A total of $17.5 \mathrm{~g}$ of ${ }^{82} \mathrm{Se}$ and $10 \mathrm{~g}$ of cach of the other isotopes was used. The isotopic enrichment for each target was greater than $96 \%$. The target material, in the form of elemental powder, was mounted in small cells, with an internal area of $10 \mathrm{~cm} \times 2.3 \mathrm{~cm}$. (Each cell was slightly wider than the nominal width of the EPICS beam.) The cells were constructed using a frame of $1 / 32$-in sheet aluminum and 0.001 -in mylar windows. The windows were attached to the frame using double-sided scotch tape, with the rear window secured over the complete area of the frame, and the front window not attached to the upper edge of the frame-allowing air to flow freely while pumping the target chamber. After packing, the typical depth of selenium was $1.7 \mathrm{~cm}$, and the ty ${ }_{t}$ ical areal density of the selenium was $600 \mathrm{mg} \mathrm{cm}^{-2}$. After packing, each cell was wrapped in a loose envelope of 0.0001-in mylar. No more than $10 \mathrm{~g}$ of isotope was put in each cell. Consequently, the ${ }^{82} \mathrm{Se}$ was put into two cells.

Each cell had a total height of $2.7 \mathrm{~cm}$, so there was room to put all five cells in the EPICS beam at the same time. Event-by-event ray tracing allows us to determine which target cell was associated with any given detected pion. Figure 1 shows a vertical cut through the target. The regions of each isotope, and aiso the aluminum frames of the cells, are clearly seen.

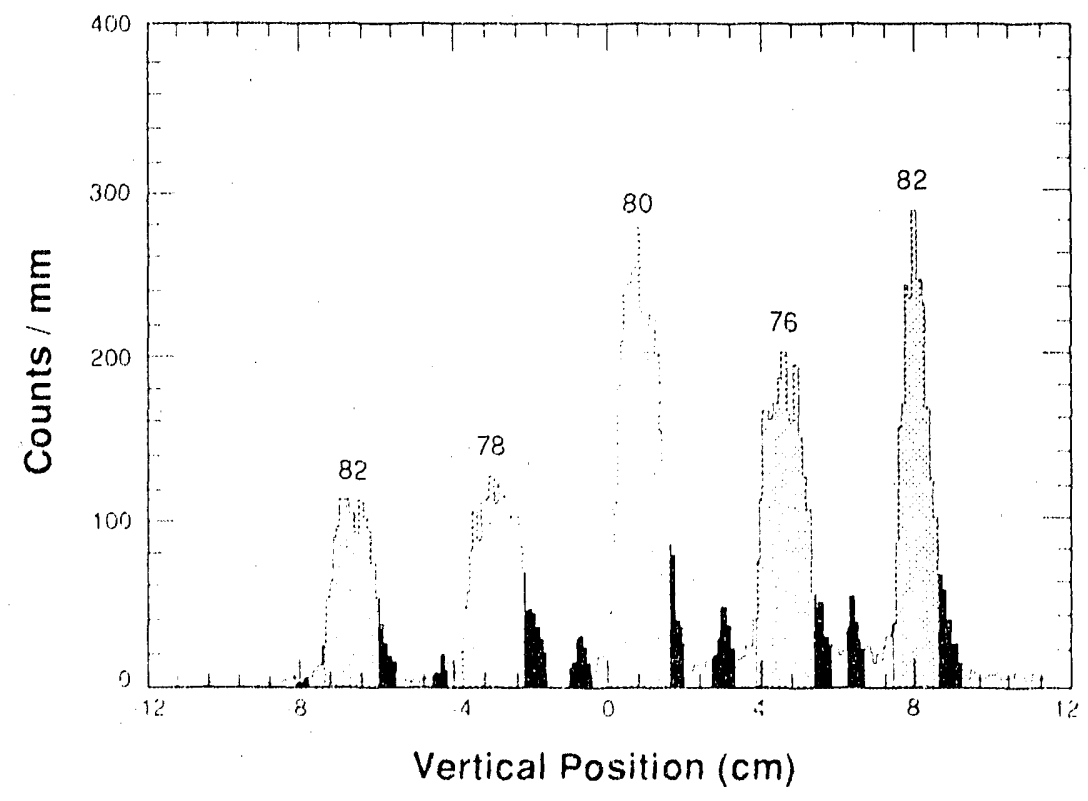

Fig. 1. Histogram of the vertical position from the target, for each event. The positive direction is vertically down. Light shading indicates selenium in the target, and black indicates the location of the frames of the aluminum cells. Each region of selenium is labeled by the mass number of the isolope. 
Preliminary results for the DIAS cross section, at $5^{\circ}$, are presented in Fig. 2. A thorough aralysis of the data, taking care to eliminate events from the aluminum frame, is in progress. The figure presents the data as a ratio to the cross section expected from a global fit to DIAS systematics. ${ }^{1}$ The systematic estimate is given by

$$
\frac{d \sigma}{d \Omega}=f(N-Z)(N-Z-1)\left(\frac{A}{42}\right)^{-\alpha},
$$

with $f=0.068 \mu \mathrm{b} / \mathrm{sr}$, and $\alpha=3.24$. It is seen that the data are not consistent with a simple dependence on $A$ and the number of excess neutron pairs. This may be indicative of effects on the reaction amplitude because of nuclear structure of the target ground state and the DIAS. A more detailed analysis, using a variety of models, will be performed when, the final numbers have been extracted from the data. We notice that if the preliminary DIAS results are used to extract the two amplitudes in the generalized seniority model of $D C X,{ }^{2}$ then the fitted shortrange amplitude ( $B$ in Ref. 2 ) overpredicts the amplitude for DCX transitions to the ground states. This result is sensitive to some known reaction mechanism effects (c.g., distortions) which have not yet been included.

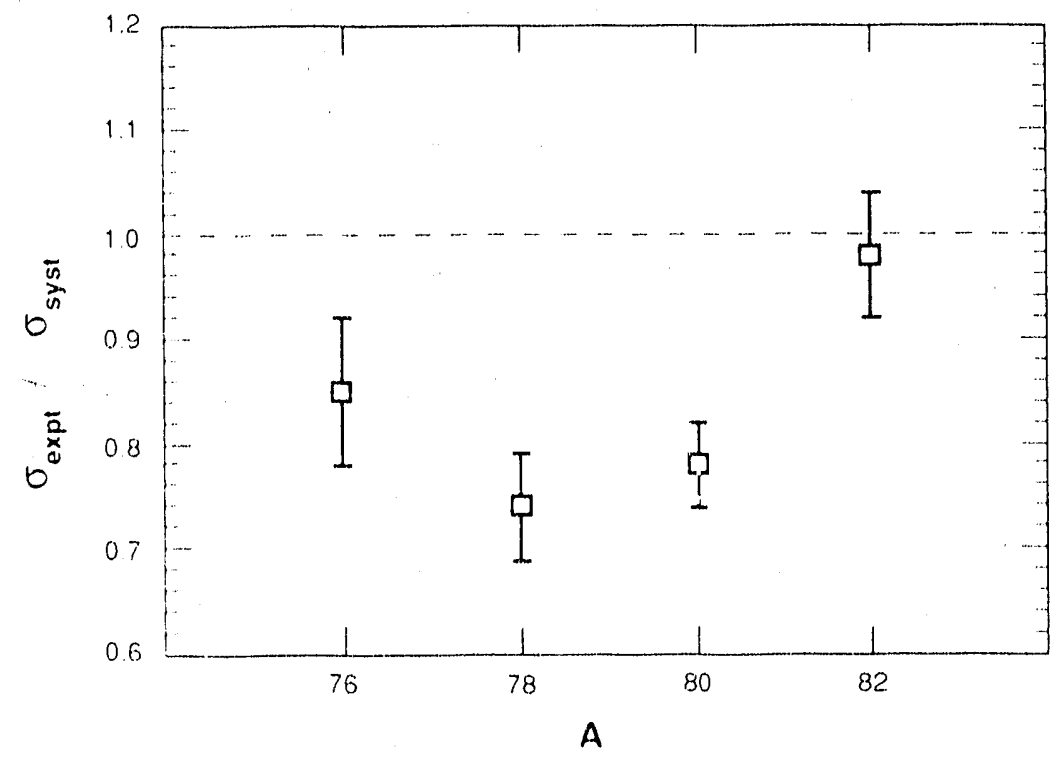

Fig. 2. The measured cross section for each isotope is plotted as a ratio to a systematic estimate discussed in the text. 
RESEARCH

Nuclear and Particle Physics

\section{References}

1. R. Gilman, H. T. Fortune, J. D. Zumbro, C. M. Laymon, G. R. Burleson, J. A. Faucett et al., Phys. Rev. C 35, 1334 (1987).

2. N. Aucrbach, W. R. Gibbs, Joseph N. Ginocchio, and W. B. Kaufmann, Phys. Rev. C 38, 1277 (1988). 
EXPERIMENTS 581 AND 704 - Fermilab

\section{Polarized Proton and Antiproton Experi- ments at Fermilab}

Argonne; Fermilab; Institute of High Energy Physics (USSR); Univ. of lowa; Kyoto Univ., Kyoto Univ. of Education, Univ. of Occupational and Environmental Health (Japan); Saclay, Institut National de Physique des Nucleaires et de Physique des Particules (France); Los Alamos; Univ. of Minnesota; Northwestern Univ.; Rice Univ.; Univ. of Udine, Univ. of Trieste, Univ. of Messina (Italy)

Spokesman: A. Yokosawa (Argonne)

Partlcipants: N. Akchurin, D. Carey, R. Coleman, M. Corcoran, J. Cossairt, A. Derevschikov, M. Gazzaly, D. Grosnick, D. HIII, K. Imal, K. Vuroda, M. Laghal, $F$. Lehar, $A$. de Lesquen, $D$. Loplano, F. Luehring, A. Maki, S. Makino, A. Masaike, Yu. Matulenko, A. Meschanin, A. Michalowicz, D. Miller, K. Miyake, T. Nagamine, F. Ness/Tedaldi, M. Nessl, C. Nouyen, S. Nunushev, $Y$. Ohashi, $Y$. Onel, G. Paulefta, A. Ponzo, G. Phillips, A. Read, N. Roberts, L. van Rossum, G. Salvato, P. Scihlavon, T. Shima, V. Solovyanov, H. Spinka, R. Stanlek, R. Takashima, $F$. Takeutchl, N. Tamura, N. Tanaka, D. Underwood, A. Vasiliev, A. Villari, J. White, A. Yokosawa, T. Yoshida, and A. Zanett
Experiments 581 and 704 at the Fermilab are for the design and construction of the high-energy polarized proton beam line of up to $200 \mathrm{GeV} / c$ (Ref. 1) and a study, based in part on the fact that there are already several experimental indications that spin effects are significant at high energies:

1. Mrasurements of $\pi^{0}$ production at high $P_{\perp}\left(P_{\perp}>2.0 \mathrm{GeV} / c\right)$ in protonproton collisions at CERN and $\pi^{-}$-proton collisions at Serpukhov revealed sizable asymmetries at $24 \mathrm{GcV} / c$ and $40 \mathrm{GeV} / c$, respectively.

2. Hyperons produced at large $x_{r}$ inclusively of nuclei and hydrogen at CERN, Fermilab, and ISR were observed to have high polarization.

3. Inelastic scattering of longitudinally polarized electrons on longitudinally polarized protons at SLAC yielded a large asymmetry, implying that proton helicity orientation is communicated to the constituent quarks.

5. Results of new EMC measurements indicate that the proton spin may not be attributable to the helicity of its constituent quarks.

Most of the proton spin may be caused by gluons and/or orbital angular momentum. To understand the basic question of the proton spin, hadron-produc: on measurements at high $P_{\perp}$ with longitudinally polarized protons on longitudinally polarized target protons seem to be very desirable.

The polarized beam facility was operated for the first time during the last run cycle. An extracted beam from the Tevatron is delivered through the MP primary beam line to the Meson Detector Building where a 0.73-interaction-length $\mathrm{Be}$ target is utilized to produce $\Lambda^{0}$ and $\bar{\Lambda}^{0}$ at $0_{\mathrm{c} . \mathrm{m}} \approx 0^{\circ}$. Protons and antiprotons from the $\Lambda^{0}$ and $\bar{\Lambda}^{0}$ decays, respectively, are brought to a final target position in the MP Hall through the MP secondary beam line $(200 \mathrm{GeV} /$ c) (see Fig. 1).

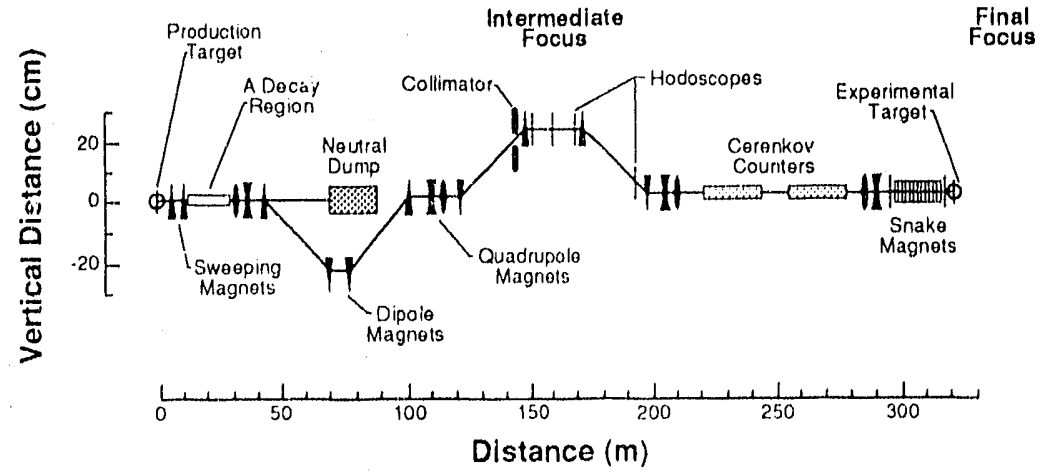

Fig. 1. Layout of elements along the MP polarized beam line. Shown here are a side view of the production target, neutral particle dump, adjustable collimator, beam-tagging region, snake magnets, Cerenkov wunters, and experimental target. Note the difference in scale between the horizontal and vertical axes. 
The polarization comes from the parity violating decays of $\Lambda^{0 \prime} s .^{2}$ The protons (antiprotons) from $\Lambda^{0} \rightarrow p \pi^{-}\left(\bar{\Lambda}^{0} \rightarrow \bar{p} \pi^{+}\right)$decay have polarization of $64 \%$ along the direction in the $\Lambda_{\text {c.m. }}$. We use $\Lambda^{0 \prime} \mathrm{s}\left(\ddot{\Lambda}^{0 \prime} \mathrm{s}\right)$ produced at the low $P_{\perp}$, where the production cross section is maximum and the $\Lambda^{0 \prime} s\left(\bar{\Lambda}^{0 \prime} s\right)$ are unpolarized. The $\vec{S}$ type beam was extracted and reaches the snake magnets, which makes fast reversal-typically every 10 spills of the spin direction. The snake magnets can also change the spin direction from $\vec{S}$ to $\vec{N}$ or $\vec{S}$ to $\vec{L}$ without altering the beam direction and phase space before and after the magnets. These magnets are expected to control systematic errors by periodic reversal. Usable pro'ons and antiprotons were selected by their tagged position corresponding to a calculated polarization magnitude of $>35 \%$ equivalent to the average polarization of $45 \%$. Two threshold Cerenkov counters were used upstream of the snake magnets to reject pions in the beam. Measurements of the beam flux for $1 \times 10^{12}$ incident protons per 20-s spill are shown in Table I.

Table I. Measurements of the Beam Flux for $1 \times 10^{12}$ Incident Protons per 20-s Spill.

\begin{tabular}{lccc}
\hline$\left(P_{\text {pav }}=45 \%\right)$ & $\begin{array}{l}\text { Tagged Beam } \\
\text { (Antiprotons) }\end{array}$ & Total Protons & Background $\pi$ \\
\hline Protons & $8 \times 10^{6}$ & $1.5 \times 10^{7}$ & $1.5 \times 10^{6}$ \\
Antiprotons & $4 \times 10^{5}$ & $7.5 \times 10^{5}$ & $3.8 \times 10^{6}$ \\
\hline
\end{tabular}

Detectors in the MP experimental hall, including beam hodoscope, transmission hodoscope, multiwire proportional chambers, lead-glass calorimeters, etc., are shown in Fig. 2.

Two polarimeters were used to measure the absolute polarization and to confirm the validity of the tagging method. One is the Primakoff-Effect Polarimeter, ${ }^{3}$ where the analyzing power for dissociation of an incoming high-energy proton into a $(\pi N)$ system by the Coulomb field of a heavy nucleus can be related to the known low-energy polarized target asymmetry in photoproduction of the corresponding $(\pi N)$ system. Asymmetries in the low energy photoproduction are quite large, up to $90 \%$ in certain kinematic regions. The same analyzing power in the corresponding kinematic region will occur in the proton-induced process at high energy. Using a $3-\mathrm{mm}$ lead target, a lead glass calorimeter, and a magnetic spectrometer, it was possible to observe the Primakoff events in the $|t|<$ $10^{-3}(\mathrm{GeV} / c)^{2}$ region and the mass range, $1.34<M\left(p, \pi^{0}\right)<1.50 \mathrm{GeV}$. About 3000 events using protons with tagged average polarizations of $45 \%$ were collected in 48 hours. The beam polarization was determined to be $(40 \pm 10) \%$, in agreement with the designed calculations. No asymmetries were found in kinematic regions where the low-energy photoproduction data show a zero analyzing power. 


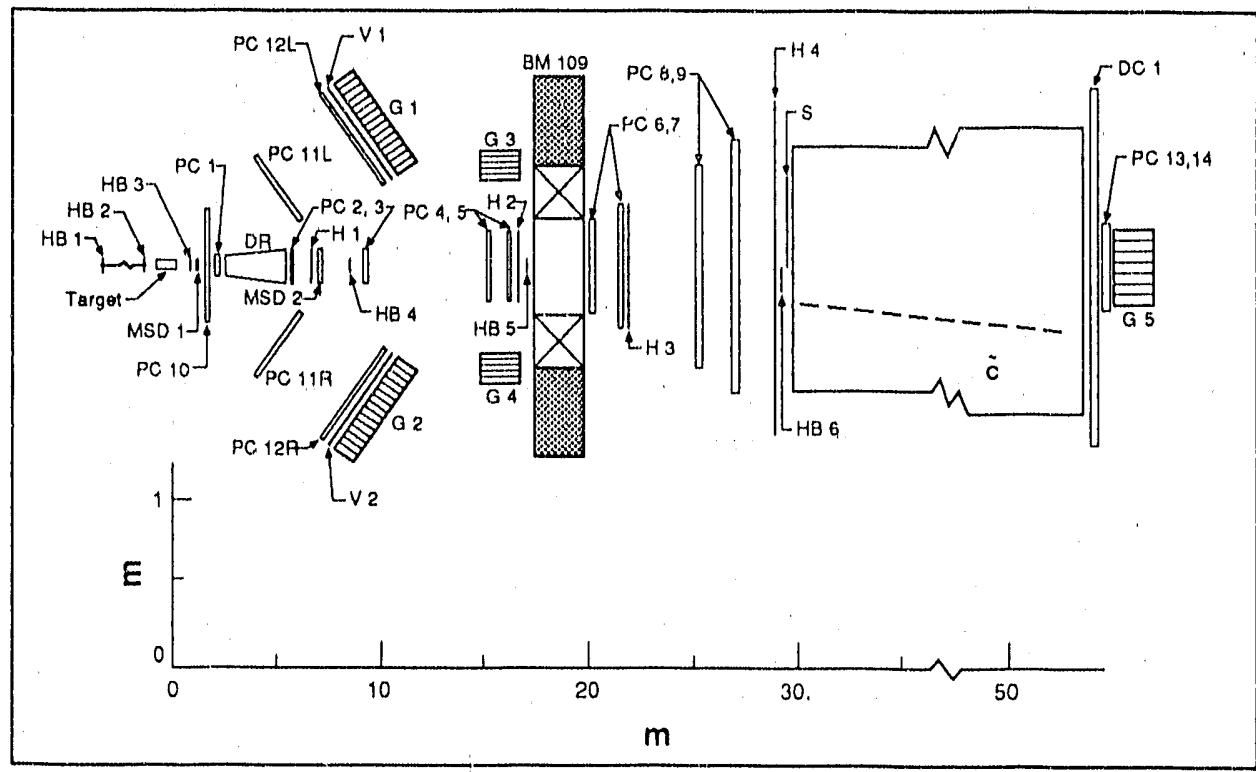

Fig. 2. Detectors in the MP experimental hall (top view). HB1,2,3: beam hodoscope. HB4,5,6: transmission hodoscope. Target: polarized proton/hydrogen target/polarimeter target. H1 to H4: trigger hodoscope. MSD1,2: multistrip detector, PC1 to 14, MWPC. V1,2: veto hodoscope. G1,2: lead glass calorimeter. G3,4: lead glass hodoscope.

Another polarimeter used was the Coulomb-Nuclear Polarimeter ${ }^{4}$, based on the interference effect in $p p(\bar{p} p)$ elastic scattering in the Coulomb nuclear interference region. Data with the polarized-proton beam were taken during two different periods, accumulating a total of $3 \times 10^{6}$ events in 29 hours. The measured result for the polarization is $41 \pm 26 \%$, compared to the beam tagged value of $42 \%$. A small amount of data was taken using the polarized antiproton beam. Assuming that the analyzing power for inclusive small-angle scattering of antiprotc: is the same as that for protons, the measured antiproton beam polarization was $47 \pm 16 \%$, compared to an expected value of $43 \%$ from the beam tagging. The results from both polarimeters, although limited by low statistics, is consisient with the expected average beam polarization as given by the beam-tagging system.

Along with the measurements made by the two polarimeters, the inclusive production of neutral pion at large $x_{\mathrm{F}}$ has also shown a spin-dependent asymmetry in the production cross section..$^{5}$ A nonvanishing analyzing power found in this inclusive reaction means that it can be used as an on-line beam polarization monitor for future experiments. Several features male this an attractive choice:

1. The reaction has a large cross section,

2. Detection of the two photons from $\pi^{0}$ decay is a relatively simple measurement, 
3. No charged-particle track reconstruction is necessary, and

4. This detector does not interfere with detectors from the other experiment in this set-up.

The average analyzing power measured for the inclusive $\pi^{0}$ production at $\left\langle x_{\mathrm{F}}\right\rangle=0.52$ and $\left\langle P_{\mathrm{T}}\right\rangle=0.8 \mathrm{GeV} / \mathrm{c}$ is $10 \pm 3 \%$.

During the next run cycles of Fermilab, $\Delta \sigma_{L}(p p)$ and $\sigma(\bar{p} p)$ will be explored, as well as hadron production on a hydrogen target with polarized beam, such as

$$
\vec{p} p \rightarrow\left(\pi^{0}, \pi^{ \pm}, \Lambda^{0}, \Sigma\right)+X
$$

\section{References}

1. D. P. Grosnick, D. A.. Hill, M. R. Laghai, D. Lopiano, Y. Ohashi, T. Shima et al., "The Design and Performance of the FNAL High-Energy PolarizedBeam Facility," Los Alamos National Laboratory document LA-UR-89-2822 (1989).

2. J. W. Cronin and O. E. Overseth, Phys. Rev. C129, 1795 (1963); O. E. Overseth and R. F. Roth, Phys. Rev. Lett. 19, 391 (1967).

3. D. C. Carey, R. N. Coleman, M. D. Corcoran, J. D. Cossairt, A. A. Derevschikov, and D. P. Grosnick, Phys. Rev. Lett. 64, 357 (1990); D. C. Carey, R. N. Coleman, M. D. Corcoran, J. D. Cossairt, A. A. Derevschikov, D. P. Grosnick et al., Los Alamos National Laboratory document LA-UR-89-3459 (1989).

4. N. Akchurin, D. Carcy, R. Coleman, M. D. Corcoran, J. D. Cossairt, A. Derevschikov, Phys. Lett. B 229, 299 (1989); N. Akchurin, D. Carey, R. Coleman, M. D. Corcoran, J. D. Cossairt, A. Derevschikov, "Analyzing-Power Measurements of Coulomb-Nuclear Interference with the Polarized-Proton and Antiproton Beams at $185 \mathrm{GeV} / c_{1}^{\prime \prime}$ Los Alamos National Laboratory document LA-UR-89-2604 (1989).

5. B. E. Bonner, J. A. Buchanan, D. C. Carcy, J. M. Clement, R. N. Coleman, M. D. Corcoran, Phys. Rev. Lett. 61, 1918 (1988). 
EXPERIMENT 772 - Fermilab

\section{Study of the Nuclear Antiquark Sea with the Drell-Yan Process at $800 \mathrm{GeV}$}

\author{
Los Alamos, Fermilab, Univ. \\ of Illinois at 'ihicago, \\ Northern Illinois Univ., \\ SUNY at Stony Brook, \\ Case Western Roserve \\ Univ., Univ. of Texas at \\ Austin \\ Spokesman: J. M. Moss (Los \\ Alamos) \\ Partlcipants: D. M. Alde, H.W. \\ Baer, T. A. Carey, G. T. Garvey, \\ A. Klein, C. Lee, M. J. Leiltch, J. \\ W. Lillberg, P. L. McGaughey, \\ C. S. Mishra, J. M. Moss, \\ J. C. Peng, C. N. Brown, \\ W. E. Cooper, Y. t. Hsiung, \\ M. R. Adams, R. Guo, I. M. \\ Kaplan, R. L. McCanthy, G. \\ Danner, M. J. Wang, M. L. \\ Barlett, and G. W. Hofimann
}

Deep inelastic lepton scattering (DIIS) from nuclei has revealed that the structure function per nucleon undergoes systematic modification as a function of the nucleon number $A .^{1-6}$ As $A$ increases, there is an increasing depletion in the relative fraction of quarks carrying a fraction of momentum $x$, both at intermediate values of $x(0.6 \geq x \geq 0.3)$ and at small values of $x(x \leq 0.1)$. The depletion in the intermediate $x$ region is often referred to as the EMC effect. In spite of many years of intense effort, there is still no consensus as to the origin of this depletion. The nuclear suppression at $x<0.1$ is equally mysterious and further is not as well characterized experimentally.

In charged DILS, a quark structure function $\left[F_{2}(x)\right]$ is measured in which each quark type is sampled proportional to the square of its charge. There is therefore no separation of the scattering on sea and valence quarks. To effect this separation, our collaboration (Exp. 772) has measured ${ }^{7}$ precise ratios of the Drell-Yan (DY) yicld for a variety of atomic nuclei in a kinematic regime that is predominantly sensitive to the sea quark distribution of the target.

The Drell-Yan cross section can be written ${ }^{8}$ as

$$
\frac{d^{2} \sigma}{d M^{2} d x_{F}}=\frac{K 4 \pi \alpha^{2}}{9 M^{4}} \frac{x_{1} \cdot x_{2}}{x_{1}+x_{2}} \sum_{i} Q_{i}^{2}\left[q_{i}\left(x_{1}\right) \bar{q}_{i}\left(x_{2}\right)+\bar{q}_{i}\left(x_{1}\right) q_{i}\left(x_{2}\right)\right],
$$

where $M^{2}$ is the mass squared of the dilepton pair $M^{2}=s x_{1} x_{2} ; x_{1}\left(x_{2}\right)$ refers to the momentum fraction of the interacting quark in the beam (target), $x_{F}=x_{1}-x_{2}$; and $s$ is the square of center-of-mass energy of the colliding two nucleons. The charge of the quarks with flavor $i$ is $Q_{i}$, and $K$ is a factor that accounts for the QCD corrections to the cross section. In Exp. 772 we select $x_{1}$ to be sufficiently large so that $q\left(x_{1}\right) \bar{q}\left(x_{2}\right) \gg \bar{q}\left(x_{1}\right) q\left(x_{2}\right)$. The ratios of the Drell-Yan yields for different targets then provide a direct measure of the $A$ dependence of the target antiquark distribution functions. Previous Drell-Yan experiments ${ }^{9,10}$ have been carried out on nuclear targets, $b$, not with sufficient statistical or systematic precision to bear on the EMC effer.

In Exp. 772 we recorded over 4' 0,000 DY muon pairs from the bombardment of targets of natural carbon, calcium, iron, tungsten, and deuterium with 800 $\mathrm{GeV}$ protons. The measurements were performed in 1987-1988 using a modified ; ersion of the large spectrometer in the Meson ast beamline at Fermilab, which was originally constructed for Exp. 605. ${ }^{11}$ The nagnetic fields of the thice dipole magnets of the spectrometer could be configured to optimize acceptance for different regions of dimuon mass. In Exp. 772 three configurations were chosen, which maximized the yields at $4.5,6$, and $8.5 \mathrm{GeV}$, respectively. The spectrometer was used in the closed-aperture configuration, permitting the use of a very highintensity primary $800-\mathrm{GeV}$ proton beam. Maximum intensities per 20-s spill varied from $2 \times 10^{12}$ protons for the high-mass setting to $0.5 \times 10^{12}$ pretons for the low-mass setting. Approximately $5.5 \mathrm{~m}$ downstream of the target, the beam was stopped in a water-cooled copper beam dump located inside a 15-m dipole magnet (the largest of the three). Beyond the beam dump, at the downstream end of the 15-m dipole, was a hadron absorber constructed of copper, graphite, and borated polyethylene blocks. 
The Exp. 772 trigger consisted of a pair of triple coincidences of hodoscope elements having the topology of a $\mu^{+} \mu^{-}$pair from the target. This trigger reduced the primary background events, consisting of random coincidences from low $p_{T}$ single muons from the beam dump, by a factor of approximately $10^{3}$. Typically, 1000 events per spill were written to tape, of which 20 were valid dimuon events from the target. Electronic live time during beam spills was kept above $98 \%$. Table I shows the integrated luminosity and number of analyzed events at each spectrometer setting.

Table I. Experiment 772 Drell-Yan Data Summary.

\begin{tabular}{lccc}
\hline Magnet Setting & Targets & $\begin{array}{c}\text { Integrated } \\
\text { Luminosity } \\
\left(\mathrm{cm}^{-2}\right)\end{array}$ & $\begin{array}{c}\text { Drell-Yan Dimuons } \\
(4<M<9 \text { or } \\
M>11 \mathrm{GeV})\end{array}$ \\
\hline High Mass & ${ }^{2} \mathrm{H}, \mathrm{Ca}, \mathrm{Fe}$ & $9.6 \times 10^{40}$ & $17 \times 10^{3}$ \\
Medium Mass & ${ }^{2} \mathrm{H}, \mathrm{C}, \mathrm{Ca}, \mathrm{Fe}, \mathrm{W}$ & $20.0 \times 10^{40}$ & $309 \times 10^{3}$ \\
Low Mass & ${ }^{2} \mathrm{H}, \mathrm{C}, \mathrm{Ca}, \mathrm{Fe}, \mathrm{W}$ & $4.9 \times 10^{40}$ & $119 \times 10^{3}$ \\
& & $3.5 \times 10^{41}$ & $445 \times 10^{3}$ \\
\hline
\end{tabular}

Beam intensity was continuously monitored by two calibrated secondary emission monitors, as well as a quarter-wav' cavity monitor. In addition, two four-element scintillator teiescopes viewing ine target at $90^{\circ}$ monitored the luminosity, the duty factor of the beam, and the detector live time.

A key element in reducing errors caused by long-term drifts and changes in efficiency was the regular, rapid interchange of targets. During stable running conditions, a cycle of two or three targets, one being ${ }^{2} \mathrm{H}$, was alternately inserted into the beam for periods of approximately $15 \mathrm{~min}$.

The solid targets ${ }^{12}$ consisted of precisely milled $7.62-\mathrm{cm}$ diameter disks, which were distributed over a length of about $50 \mathrm{~cm}$, to approximate the geometry of the liquid deuterium cell. Target thicknesses were chosen to roughly equalize trigger rates with the ${ }^{2} \mathrm{H}$ cell, ranging from $6 \%(\mathrm{~W})$ to $15 \%\left({ }^{2} \mathrm{H}\right)$ of an interaction length. The vapor pressure in the cryogenic cell (hence the density of the liquid) was continuously monitored and written to tape at the end of each spill. Elemental assays of all targets and beam attenuation factors were included as part of the final calculation of luminosity.

Because of the extremely high instantaneous luminosities (up to $10^{36} \mathrm{~cm}^{-2} \mathrm{~s}^{-1}$ ), there were some dimuon pairs produced from random coincidences of high $p_{T}$ muons. These events, constituting approximately $3 \%$ of the data, were removed using the observed like-sign muon pairs as a measure of the random coincidence yield.

Track reconstruction was performed using modified versions of the Exp. 605 software running on a Fermilab-designed Advanced Computing Project parallel 
computer system. Such a system, having 43 nodes, was installed at the LAMPF Data Analysis Center. The tracker was optimized to give the highest possible reconstruction efficiency, which averaged approximately $91 \%$. The loss in efficiency was found to be directly proportiona? to the instantaneous luminosity. Target-to-target differences in the reconstruction efficiency were computed using studies of this rate dependence, and corrections were applied.

The estimated systematic error in the overall normalization is $\sim 10 \%$. The systematic error in the ratios of the yields from the solid targets vs deuterium are: the rate dependence $(1.5 \%)$; acceptance $(0.4 \%)$; deuterium thickness $(0.4 \%)$; and beam attenuation $(0.3 \%)$. All other contributions are negligible. This results in a total estimated systematic error in the ratios of less than $2 \%$.

Acceptance-corrected mass spectra from the three spectrometer settings are shown for the ${ }^{2} \mathrm{H}$ target in Fig. 1, along with a calculation of the DY cross section in the leading-log approximation $\left[q(x) \rightarrow q\left(x, M^{2}\right)\right]$. Using the structure functions of Eichten et al., ${ }^{13}$ one obtains a $k$ factor of $2.2 \pm 0.2$ by normalizing to the data.

Figure 2 shows ratios of the Drell-Yan yields $2 \times Y_{A} / A \times Y_{2 H}$ for each heavy target as a function of $x_{2}$ for muon pairs with positive $x_{F}$. The mean $x_{F}$ in the

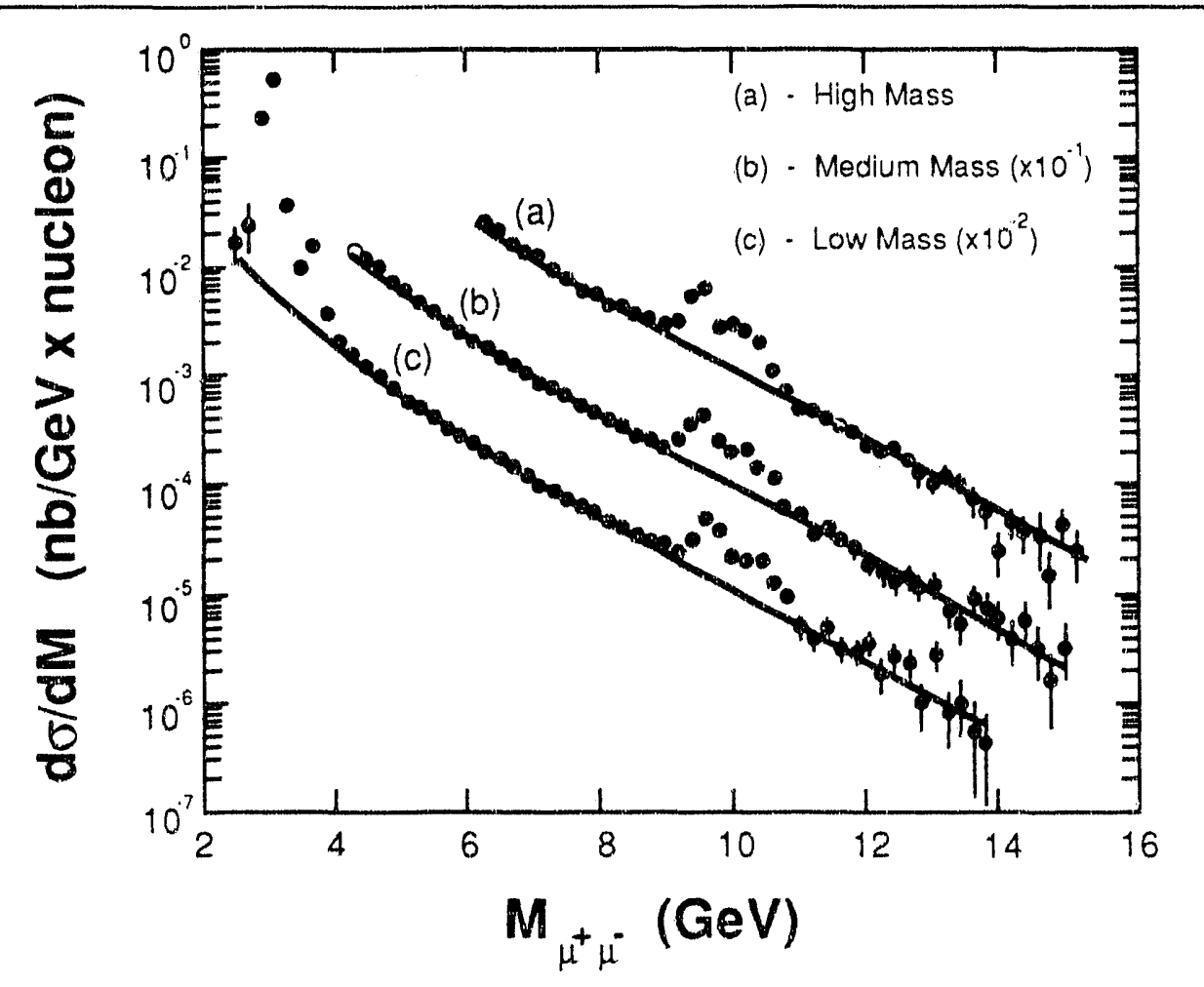

Fig. 1. Acceptance-corrected mass spectra for ${ }^{2} \mathrm{H}$ from the three spectrometer settings. The solid curves are calculations of the Drell-Yan cross section using the structure functions of Eichten et al. ${ }^{12}$ 


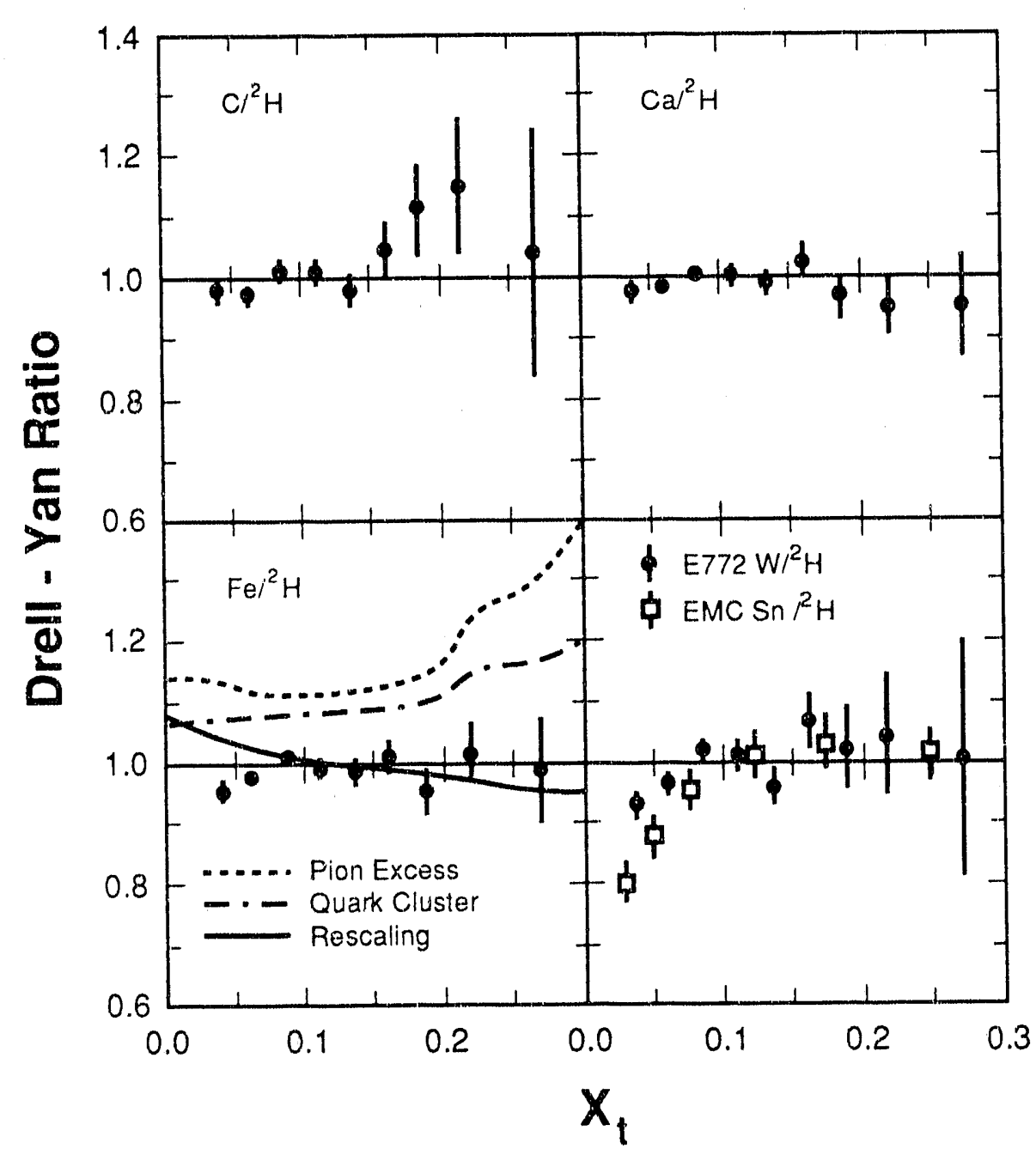

Fig. 2. Ratios of Drell-Yan yields $2 \times Y_{A} / A \times Y_{2 H}$ for positive $x_{F}$. The curves shown with $\mathrm{Fe} / 2 \mathrm{H}$ are predictions from various models of the EMC effect. Also shown are the DILS data for $S n /{ }^{2} \mathrm{H}$ from the EMC group. ${ }^{4}$

data set is 0.26 . A calculation of the fraction of the accepted DY events caused by $q_{\mathrm{p}} \bar{q}_{\mathrm{t}}$ annihilation yiclds $\sim 0.95$ at $x_{2}=0.05$ and $\sim 0.75$ at $x_{2}=0.3$. Scaling violations are small over the present range of $Q^{2}\left(\mathrm{M}^{2}\right)$ and, as there is no evidence for an $A$ dependence, the data are integrated over mass. Clearly, the $A$ dependence in the antiquark structure function is small. Even the modest enhancement (approximately $5 \%$ ) seen in two DILS experiments is not present in the antiquark ratio.

The EMC effect has inspired a plethora of theoretical explanations in the past six years. Those calculations, which invoked a convolution of nonnurloonic 
degrees of freedom to explain the apparent excess of scattering probability at small $x$, will not fare well in light of the present data. The first two models discussed are in this category.

Pion Excess. This model in its earliest forms accounted for the now discredited rise in the $F_{2}^{\mathrm{Fe}} / F_{2}^{\mathrm{D}}$ ratio at small $x$, as well as for the depletion of the valence quark structure function. ${ }^{14-16}$ The small enhancement in the pion cloud surrounding a bound nucleon arises from a conjectured attractive $p$-wave $\pi-N$ interaction in nuclear matter. The strength of this interaction is often characterized by the Landau-Migdal parameter $g_{0}^{\prime}$; typical values found in the literature range around $g_{0}^{\prime} \approx 0.6-0.7$. Figure 2 compares the results of a calculation, described in detail in Ref. 17, to the present Fe $/{ }^{2} \mathrm{H}$ data. It is clear that the calculation with $g_{0}^{\prime}=0.6$ is completely inconsistent with the data. If $g_{0}^{\prime}$ is increased to 0.8 , there is little remaining pion excess and the calculation is consistent with the present data, although not with the depletion of the valence quark distribution seen in DILS.

Quark-Cl:'ster. These models view the nucleus as being composed of a combination of ordinary nucleons plus some fraction of multiquark $(6 q, 9 q$, and higher) clusters formed by the overlap of nucleons. In describing the sea-quark region, the models' most crucial assumption has to do with the momentum sharing between the $q \bar{q}$ sea and the gluon distribution. In the model of Carlson and Havens, ${ }^{18}$ for example, the gluon momentum fraction for the $6 q$ cluster is assumed to be the same as for the free nucleon. This results in a significant enhancement of the sea, even for a modest $15 \% 6 q$-cluster fraction. The calculated DY ratio (Fig. 2) is in significant disagreement with the present data. A different ${ }^{19}$ but also plausible assumption of constant ratio of momentum fraction for sea/glue, in going from nucleons to $6 q$-clusters, leads to almost no enhancement of the DY ratio for any reasonable fraction of clusters.

Rescaling. This model is based on the notion that nuclear binding results in a phenomenon that is very similar to the familiar scaling violation associated with gluon emission. ${ }^{20}$ Comparisons to the present DY data are made on the basis of the scale change of structure functions:

$$
f\left(x, Q^{2}\right)-f\left(x, \xi Q^{2}\right),
$$

where $\xi \approx 2$ over the $Q^{2}$ range of Exp. 772. The results, shown in Fig. 2, follow closely the pattern of scaling violation observed in the EMC experiments. ${ }^{1-6}$ This model gives a reasonable account of the DY ratio, except in the range $x<0.1$, where the approximations made in this model are known to break down.

In summary, this experiment shows no nuclear effect in the antiquark sea in the range $0.1<x<0.3$. Models of the EMC effect that postulate a significant pion excess or $\bar{\eta}$ enhancement in multiquark clusters are ruled out. The $Q^{2}$ rescaling model is consistent with the present data for $x \geq 0.1$.

A slight, but experimentally significant, depression of the antiquark ratio is seen in the heaviest targets for the range $x<0.1$. Figure 2 compares present data for $\mathrm{W} /{ }^{2} \mathrm{H}$ to the $F_{2}$, ratio for $\mathrm{Sn} /{ }^{2} \mathrm{H}$ from the EMC group. ${ }^{4}$ The lepton scattering 
data exhibit a more pronounced shadowing at small $x$. It is clearly of interest to know whether this difference can be understood in terms of current models of shadowing.

\section{References}

1. J. J. Aubert, G. Bassompierre, K. H. Becks, C. Best, E. Bohm, X. DeBovard et al., Phys. Lett. 123B, 275 (1983).

2. R. G. Arnold, P. E. Bosted, C. C. Chang, J. Gomez, A. T. Katramatou, G. G. Peterson et al., Phys. Rev. Lett. 52, 727 (1984).

3. G. Bari, A. C. Benvenuti, D. Bollini, G. Bruni, T. Camporesi, G. Neiman et al., Phys. Lett. 163B, 282 (1985).

4. J. Ashman, B. Badelek, G. Baum, J. Beaufays, C. P. Bee, C. Benchouk et al., Phys. Lett. 202B, 603 (1988).

5. M. Arneodo, A. Arvidson, J. J. Aubert, B. Badelek, J. Beaufays, C. P. Bee et al., Phys. Lett. 211B, 493 (1988).

6. A. Bodek, N. Giokaris, W. B. Atwood, D. H. Coward, D. J. Snerdan, D. L. Dubin ct al., Phys. Rev. Lett. 50, 1431 (1983); 51, 534 (1983).

7. D. M. Alde, H. W. Baer, T. A. Carey, G. T. Garvey, A. Klein, C. Lee et al., Phys. Rev. Lett. 64, 2479 (1990).

8. I. R. Kenyon, Rep. Prog. Phys. 45, 1261 (1982).

9. P. Bordalo, P. Busson, L. Kluberg, a. Romana, R. Salmeron, C. Ballée et al., Phys. Lett. 193B, 368 (1987); 193B, 373 (1988).

10. A. Ito, R. J. Fisk, H. Jöstlein, D. M. Kaplan, S. W. Herb, D. C. Hom et al., Phys. Rev. D 23, 604 (1981).

11. C. N. Brown, W. E. Cooper, D. Finicy, Y. B. Hsiung, A. M. Jonckheere, H. Jostlein et al., Phys. Rev. Lett. 63, 2637 (1989).

12. J. Gursky, H. Bacr, F. Flick, and D. Gallegos, Nucl. Instrum. and Methods A 282, 62 (1989).

13. E. Eichten, I. Hinchliffe, K. Lane, and C. Quigg, Rev. Mod. Phys. 56, 579 (1984); 58, 1065 (1986).

14. C. H. Llewellyn-Smith, Phys. Lett. 128B, 107 (1983).

15. M. Ericson and A. W. Thomas, Phys. Lett. 128B, 112 (1983).

16. E. L. Berger, F. Coester, and R. B. Wiringa, Phys. Rev. D 29, 398 (1984).

17. W.-Y. P. Hwang, J. M. Moss, and J. C. Peng, Phys. Rev. D 38, 2785 (1988).

18. C. E. Carlson and T. J. Havens, Phys. Rev. Lett. 51, 261 (1983). 
19. H. J. Pirner and J. P. Vary, Phys. Rev. Lett. 46, 1376 (1981).

20. F. E. Close, R. L. Jaffe, R. G. Roberts, and G. G. Ross, Phys. Rev. D 31, 1004 (1985). 
EXPERIMENT 1075 - HIRAB

\section{Interaction of Relativistic $\mathrm{H}^{-}$Ions with Matter}

Univ. of New Mexico, Los Alamos, St. Mary's College, California State Univ., Univ. of Mashad (Iran), Oswego High School (New York)

Spokesmen: H. C. Bryant and A. H. Mohagheghi (Univ. of New Mexico).

Participants: P. G. Harris, H. C. Bryant, A. H. Mohagheghl, R. A. Reeder, C. Y. Tang, J. B. Donahue, C. R. Quick, Jr., D. C. Rislove, H. Sharifian, H. Tooloonchl, and T. C. Allman
This experiment addresses the question of what happens to a relativistic $\mathrm{H}^{-}$ion when it passes through a thin foil. For the foil thicknesses we have studied, ranging from 15 to $300 \mu \mathrm{g} / \mathrm{cm}^{2}$, an appreciable fraction of the $\mathrm{H}^{-}$beam survives intact, some $\mathrm{H}^{-}$ions are stripped down to protons, and the remainder are distributed over the states of $\mathrm{H}^{0}$. This experiment is different from the lowenergy studies, in that the projectile velocity is comparable to the speed of light, leading to an interaction time typically of less than a femtosecond. During this interaction, the $\mathrm{H}^{-}$ion encounters a very strong quasi-periodic ficld. The nature of the interaction mechanisms, which could be chaotic, ${ }^{1}$ is not well understood.

The yields of low-lying states of neutral hydrogen were measured by Doppler-tuning a Nd:YAG laser to excite transitions to a Rydberg (i.c., highly excited $\mathrm{H}^{(0)}$ state which was then field-ionized in an electren spectrometer. We have data on the yields of $n=2$ at $226 \mathrm{MeV}, n=2,3$ at $500 \mathrm{MeV}, n=2,3,4$ at $581 \mathrm{MeV}, n=2$ at $716 \mathrm{MeV}$, and $n=1,2,3,4,5$ at $800 \mathrm{MeV}$ for 17 carbon foils ranging in thickness from 15 to $300 \mu \mathrm{g} / \mathrm{cm}^{2}$. Figure 1 shows the relative yield of $n=2$ at $800 \mathrm{MeV}$ as a function of foil thickness. The solid line is the best fit of the simple rate equation,

$$
\text { Yicld }=A *\left(e^{-c x}-e^{-b x}\right),
$$

where $x$ is the foil thickness. ${ }^{2}$

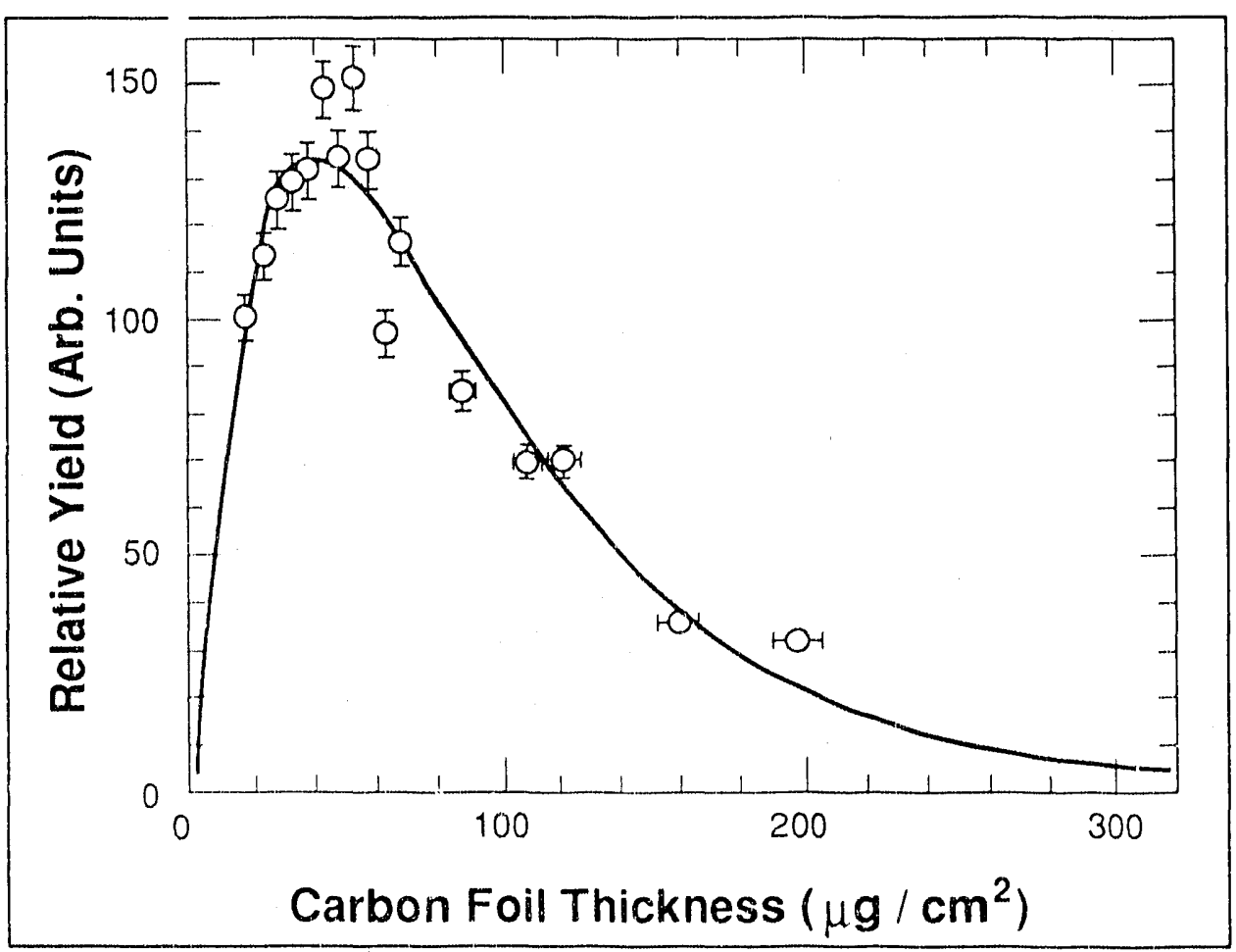

Fig. 1. Relative yield of $n=2$ at $800 \mathrm{MeV}$ as a function of carbon foil thickness. The solid line is the best fit to the simple rate equation. 
The integrated yields of $n=10+n=11$ at $800 \mathrm{MeV}$, and of $n=11+\ldots+16$ at $581 \mathrm{MeV}$ were also measured by selective field ionization for the carbon foils. In this case, spectra are formed by plotting the number of liberated electrons versus the spectrometer field as illustrated in Fig. 2. In both sases, a magnet downstream of the foil was used to sweep free electrons out cf the beam pipe, which at the sarne time selectively ionized high Rydberg states. Figure 3 displays the integrated yield of $n=10+n=11$ at $800 \mathrm{MeV}$ as a function of foil thickness. The solid line again is the best fit to the simple rate equation.

Our data seem to favor a production mechanism for excited $\mathrm{H}^{0}$ atoms by a relativistic $\mathrm{H}^{-}$berm passing through carbon foils which is, at least in part, diffusive. That is, to arrive at $\mathrm{H}^{0}(n)$ one must first make $\mathrm{H}^{(1)}(1)$, then $\mathrm{H}^{0}(2), \ldots$, etc., up to $\mathrm{H}^{0}(n)$. From this point of view, the distribution of excited states should vary with thickness, with the higher $n$ states peaking at greater thicknesses. This is in qualitative agreement with our data. At a more fundamental level, the production of each state exhibits a pronounced target-thickness dependence suggesting that the excited states are formed during the passage of the projectiles

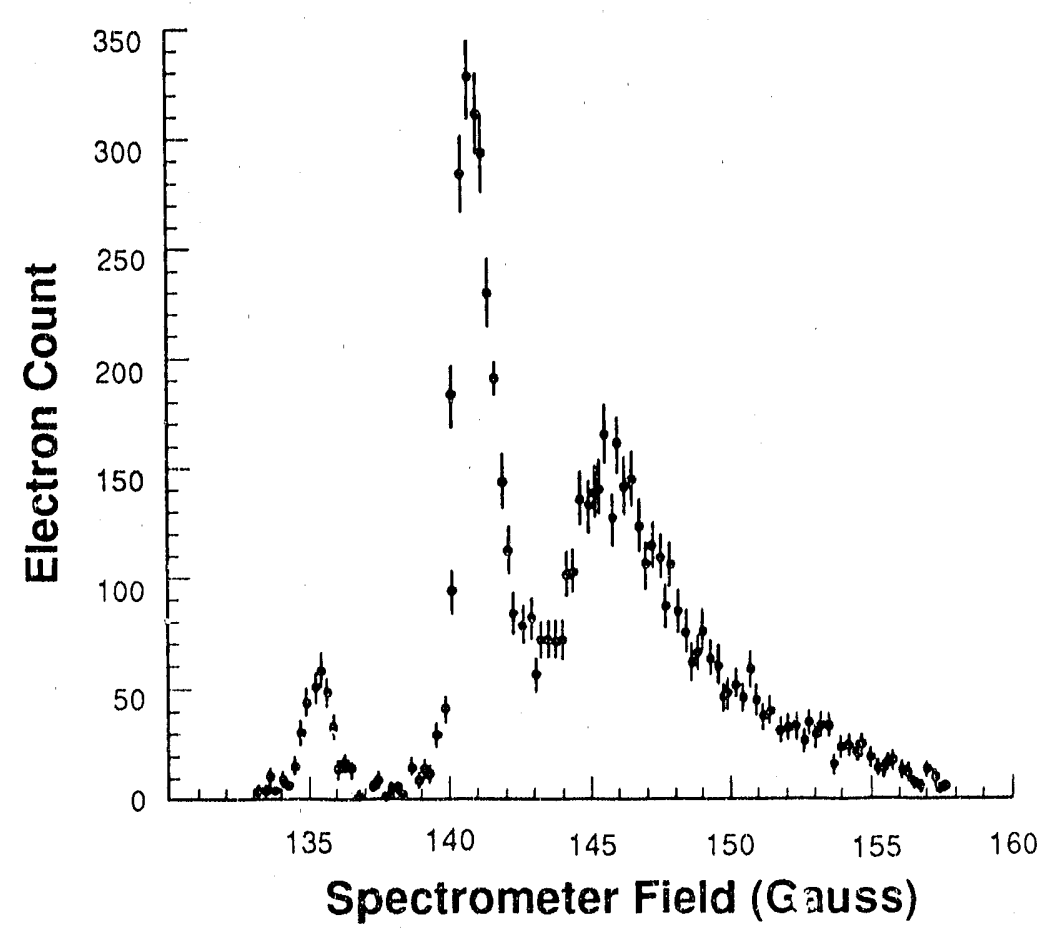

Fig. 2. The electron spectrum for a $65-\mu \mathrm{g} / \mathrm{cm}^{2}$ carbon foil. The small peak at $135.5 \mathrm{G}$ is caused by free electrons traveling along with the beam. The sharp peak at 141.0 $\mathrm{G}$ is $n=11$, and the broad peak at 147.0 is $n=10$. Higher Rydberg states are ionized by a sweep magnet located between the foil and the spectrometer and, therefore, do not show up in the spectrum. 


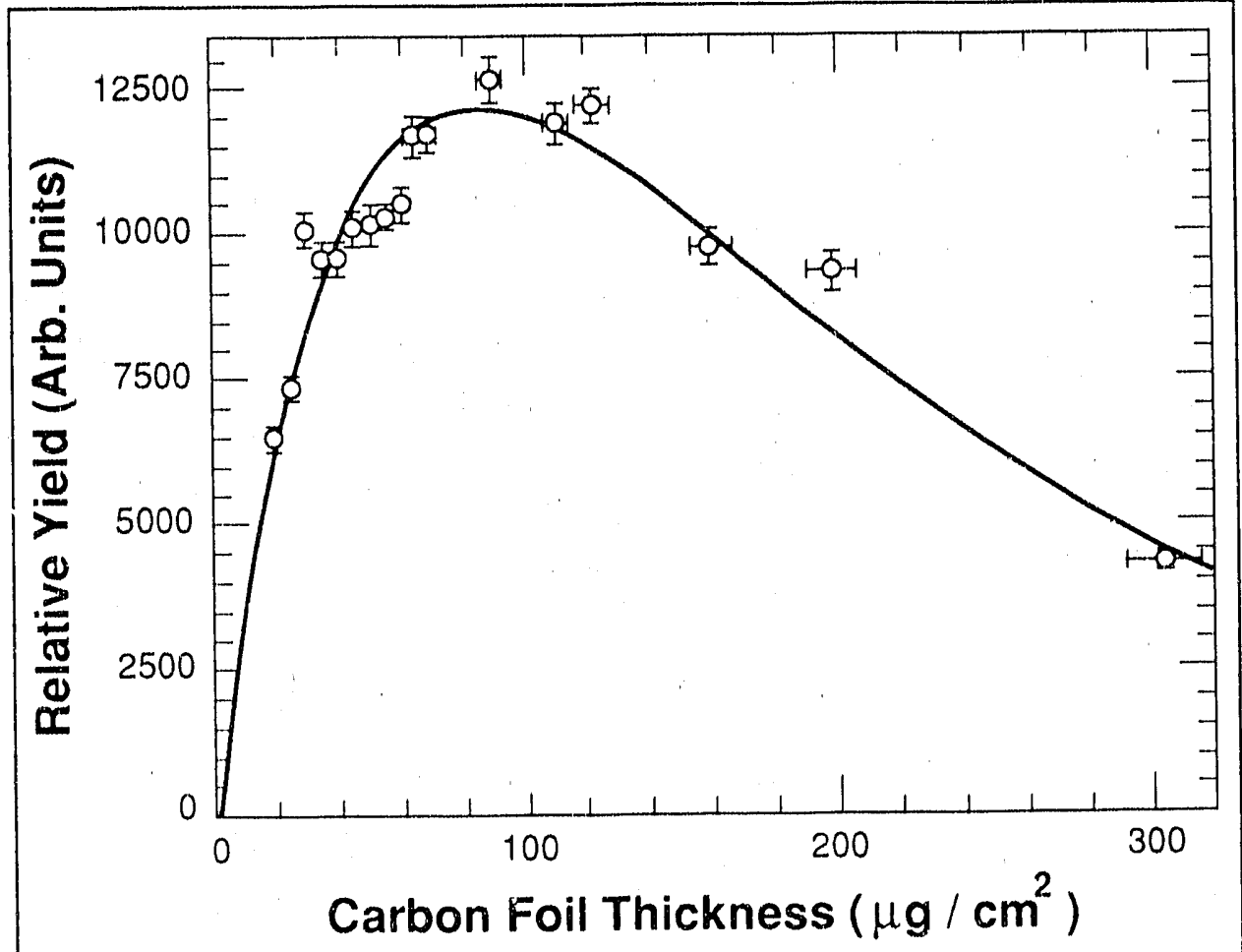

Fig. 3. Relative yield of $n=10+11$ at $800 \mathrm{MeV}$ as a function of carbon foil thickness. The solid line is the best fit to the simple rate equation. Notice that the thickness for maximum yield is $87 \mu / \mathrm{cm}^{2}$ as compared with $n=2$ yield which peaks at $42 \mu \mathrm{g} / \mathrm{cm}^{2}$.

through the foil target. This is a deviation from the low-energy models where it is assumed that excited states, which are prohibited inside a solid, are formed by Coulomb capture of target electrons from the last layer of atoms in the foil. ${ }^{3}$ A Monte Carlo simulation has been developed to transport a given distribution of excited states down the beamline and to model the field ionization of the remaining Rydberg atoms in the electron spectrometer. Extensive analysis is underway to fit the Monte Carlo results to the electron spectra by varying the initial distribution of excited states.

This experiment is supported in part by a grant from the Division of Chemical Sciences, Office of Basic Energy Sciences, Office of Energy Rescarch, U.S. Department of Energy.

\section{References}

1. J. Burgdorfer and C. Bottcher, Phys. Rev. Lett. 61, 2917 (1988). 


\section{RESEARCH}

Nuclear and Particle Physics

2. M. D. Eisenberg, "Target Thickness Data for Various Electron Stripping Targets," Los Alamos National Labnratory memorandunı AT-4/84/157, July 1984.

3. W. N. Lennard and C. L. Cocke, Nucl. Instrum. and Methods 110, 137 (1973). 


\section{EXPERIMENT 1121 - HIRAB \\ High Excitations and Double Escape in the Negative Hydrogen Ion}

Univ. of New Mexico, Los
Alamos, St. Mary's
College, California State
Univ., Univ. of Mashad
(Iran), Oswego High
School (New York)
Spokesmen: J. E. Donahue (Los
Alamos) and P. G. Harris (Unit.
of New Mexico)
Participants: P. G. Harris, H. C.
Bryant, A. H. Mohagheghi,
R. A. Reeder, C. Y. Tang,
J. B. Donahue, C. R. Quick,
Jr., D. C. Rislove, H. Shariflen,
H. Tooloonchl, and T. C. Altman

\section{Objectives}

The aim of this experiment is to study the threshold region for double photodetachment in $\mathrm{H}^{-}$, where the two electrons share the energy from a single photon, as well as the region just below this threshold, whirh is rich in autoionizing resonances. The resonances are of two principa! rypes: the first may be considered as a "core" excited hydrogen atom, which is polarized by the second, outer, electron. ${ }^{1}$ This polarization results in a potential which supports one or more bound states. An example of this situation is the so-called Feshbach resonances, of which an infinite series is believed to lie below each hydrogenic excited state. (The strongest of these, the ${ }^{1}$ po Feshbach resonance below $n=2$, was discovered by this group more than ten years ago.)

The second form of resonance is the so-called "ridge-state" resonance, ${ }^{2}$ in which the strong correlations between the electrons result in their lying on the back of a broad, flat potential ridge in hyperspherical coordinates. In this situation, they remain equidistant from the nucleus and diametrically opposite one another; they lose their association with the core hydrogen atom, and they share their energy equally. In the limit, this results in the double detachment process. The former of these resonance types should show a repetitive trend from one $\mathrm{H}^{0}$ threshold to the next; the latter should be independent of the hydrogenic energy levels.

\section{Technique}

The doubly excited resonances in $\mathrm{H}^{-}$all lie in the region from 10.9 to $14.35 \mathrm{eV}$. In order to obtain photon energies so far into the ultraviolet, light from the fourth harmonic of a Nd:YAG laser was made to intercept the relativistic $\mathrm{H}^{-}$beam at an angle, $\alpha$, which is varied by means of a system of mirrors on a turntable. The equipment is illustrated schematically in Fig. 1. The barycentric photon energy $E$ is tuned, according to the Doppler shift formula

$$
E=E_{1 a t, \gamma}(1+\beta \cos \alpha)
$$

where $\beta$ and $\gamma$ are the usual relativistic parameters, and $\alpha$ is the angle of interception of the laser and particle beams, such that $\alpha=0$ is head on.

Let us consider a photon with a Doppler-tuned energy sufficient to detach the outer electron from the $\mathrm{H}^{-}$ion and to excite the remaining hydrogen atom into the state, say, $n=4$. There is a large probability that it will, instead, eject the outer electron and leave the neutral hydrogen in $n=1, n=2$, or $n=3$. It is essential that we discriminate against such "background" events, and count only those $\mathrm{H}^{0 \prime} \mathrm{s}$ in $n=4$.

In order to do so, we make use of the relativistic transformation of magnetic ficlds,

$$
F_{\perp c m}=c \beta \gamma, B_{\perp \mid a t,} .
$$




\section{$\mathrm{H}^{-}$Beam}

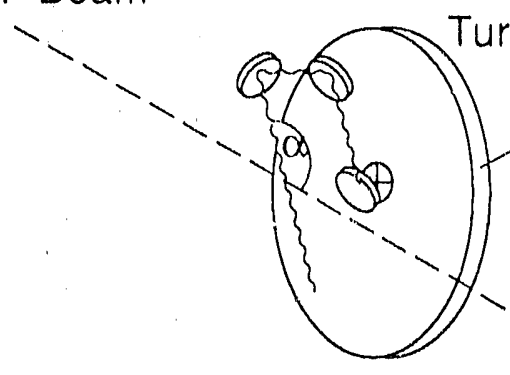

Turntable

1. $H^{-}+\gamma \rightarrow H^{0 *}+e$<smiles></smiles>
2. $H^{0 *}(n)+B \rightarrow H^{+}+e$

lonizing

Magnet

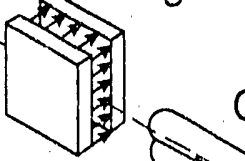

Charge Separating Magnet

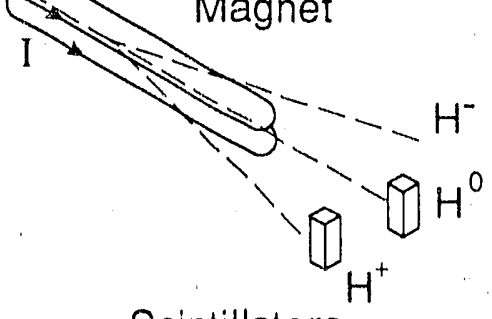

Scintillators

Fig. 1. Schematic diagram of apparatus used to observe high-lying resonances.

A hydrogen atom that is in $n=4$ or above will be field ionized - - the barycentric electric field $F$ lowering the potential barrier binding the electron, and thus releasing it-at a field strength of about $2.5 \mathrm{MV} / \mathrm{cm}$, which can be produced by applying a magnetic field of $5 \mathrm{kG}$ in the lab. The products of this process are a proton and an electron. Thus, by applying these two techniques in series, we first produce hydrogen atoms in a range of excited states $(n=1,2,3,4)$, and then strip those in $n=4$ to produce protons, which can be separated magnetically from the neutral atoms (and surviving negative ions) and detected further downstream.

We might expect, then, that as we increase the barycentric photon encrgy, starting from that needed to produce $\mathrm{H}^{n}(n \leq 3)$, we should see a staircase-type structure with the onset first of $n=4$ production, then of $n=5,6$, and so on. In addition, by reducing the magnetic field to the point where it is no longer able to strip $\mathrm{H}^{0}(n=4)$, but is able to strip $\mathrm{H}^{0}(n \geq 5)$, we should again see a staircase structure, but this time beginning with the onset of $n=5$ production. Thus, we have the means to isolate and study a single channel at a time. The amplitude for production of $\mathrm{H}^{(1 *}$ (where $*$ represents one excited electron) adds coherently to that for production of $\mathrm{H}^{-* *}$, causing the resonances to appear superimposed on the continuum of these in dividual channels. 
The double detachment threshold was to have been studied in a similar manner, except that no strong ionization magnet was required, as the signal was simply the protons produced in the photodetachment process itself. A weak magnet was used to separate the charge species to avoid producing false signals from the ionization of highly excited $\mathrm{H}^{0 *}$ states.

\section{Structure of the Resonances}

If we represent the amplitude of a resonant state as

$$
\psi_{R}(t)=A e^{-i \omega_{0} t} e^{-t / 2 \tau},
$$

a Fourier transform into the energy domain gives

$$
\psi_{R}(E)=\frac{i a \Gamma / 2}{\left(E-E_{0}\right)+i \Gamma / 2},
$$

where $\Gamma \tau=\hbar, E=\hbar \omega$, and $a=2 A \hbar / \Gamma$.

This, in turn, leads to the $\mathrm{s}$ sual Breit-Wigner formula for the cross section of a resonance,

$$
\sigma=\psi^{*} \psi=\frac{a^{2} \Gamma^{2} / 4}{\left(E-E_{0}\right)^{2}+\Gamma^{2} / 4} .
$$

If, however, the resonance lies on a continuum, then the total amplitude $\psi_{t}$ becomes, instead,

$$
\psi_{t}=b+\frac{a c^{i \phi} \Gamma / 2}{\left(E-E_{0}\right)+i \Gamma / 2},
$$

where $b$ is the continuum amplitude and $\phi$ is the phase difference between the resonance and the continuum. This leads casily to

$$
\sigma=b^{2}+\frac{a^{2}+2 a b(c \cos \phi+\sin \phi)}{\left(c^{2}+1\right)}
$$

where $c=2\left(E-E_{0}\right) / \mathrm{l}$.

The shape of the resonance therefore depends critically on the phase $\phi$ between the resonance and the continuum. The resonances in question here are interesting in that the phase difference with the continuum is close to $180^{\circ} \ldots$ they therefore appear as dips rather than peaks.

\section{Results}

A large background, as yet unexplained but possibly from the $\mathrm{H}^{-}$source itself (our group normally uses $\mathrm{P}^{-}$), meant that meaningful study of the double detachment threshold region, where by definition, the cross section is small, became impossible. However, the study of the resonances yielded results far 
beyond expectations. Up to a dozen new autoionizing resonances were discovered. Three such are clearly visible as dips in the continuum in Fig. 2, which shows the production cross section for $\mathrm{H}^{0 *}\left(n=,^{\wedge}\right)$. These dips were mapped out systematically, and their quenching in electric fiels was also observed. The data are currently being analyzed to determine how closely the energies and widths of the resonances match theoretical predictions.

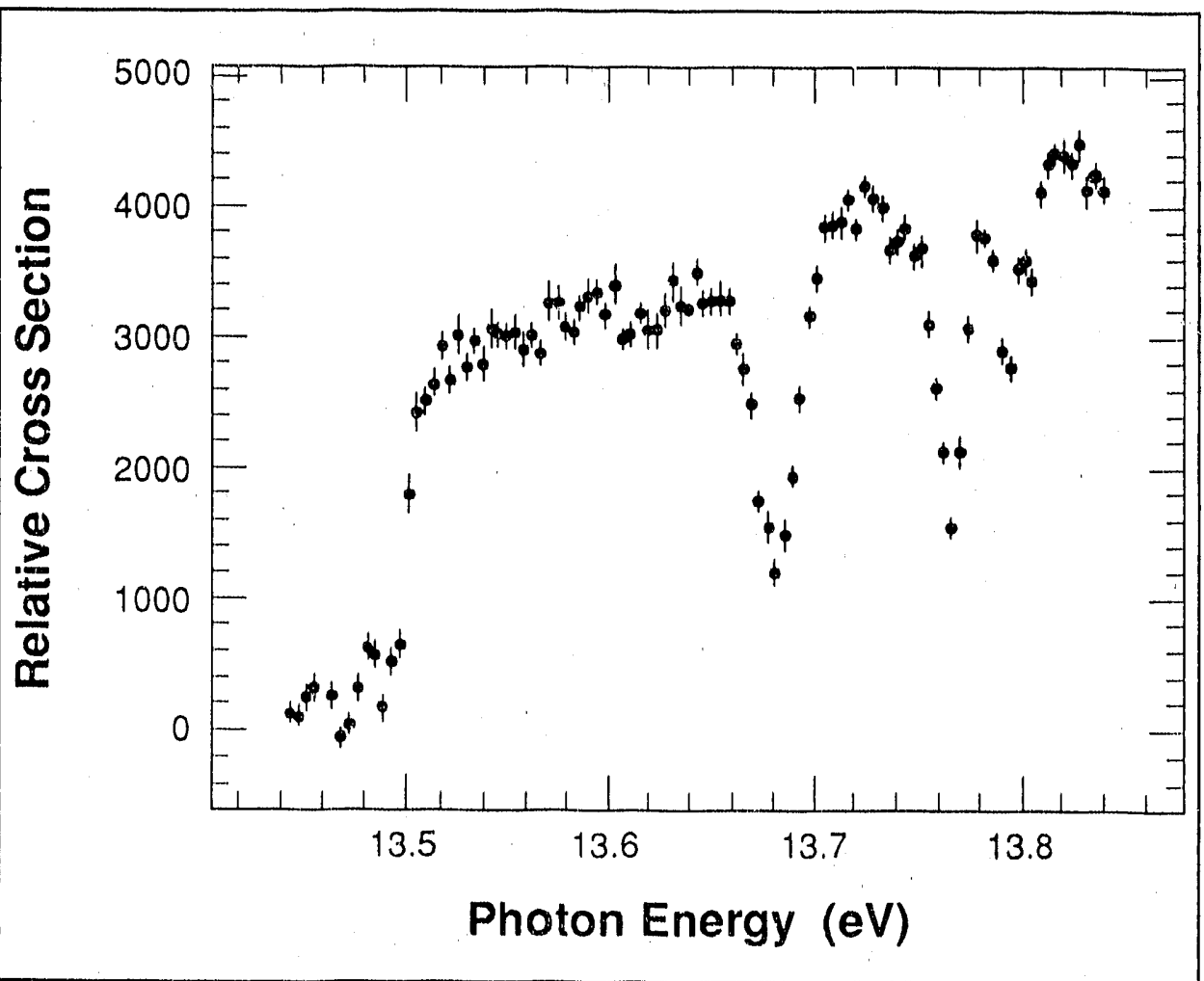

Fig. 2. Relative yield of excited $H^{n}$ atoms, with $n \geq 4$. The resonances appear as dips in the continuum cross section.

In addition, a method for improving the resolution by ruducing the momentum spread of the beam by a factor of five was successfully demonstrated. ${ }^{3}$ Again, the backgrounds prevented our utilizing this technique to the full, but it shows much promise for the future.

\section{Future Goals}

The region of high-lying resonances is a rich territory for future exploration. We now have the capability to do a much more thorough study with a new detection technique; instead of using field ionization to detect the excited neutral hydrogen atoms, they would be intercepted by a second laser, with the photon 
energy of this laser tuned to the transition from states $n$ to 13 , where $n$ is the lowlying excited state under study. Our electron spectrometer, a well-understood and reliable instrument, would fieid-ionize the $n=13$ state and detect the electron thereby produced, eliminating the need for detecting the (background-limited) proton signal at all. This would have the three major advantages of significantly extending the available range of study (in particular, to the region between $n=2$ and $n=4$, where the phase difference between the resonances and the continuum changes from $0^{\circ}$ to $180^{\circ}$ ); reducing the background levels dramatically (possibly even to zero); and further isolating the individual channels (so that, for example, above the $n=5$ threshold, the $n=4$ channel could still be detected in isolation, thereby allowing the measurement of branching ratios of the resonances into the different channels). Only minor modifications are required to our apparatus, and these are being carried out now.

This experiment is supported in part by a grant from the Division of Chemical Sciences, Office of Basic Energy Sciences, Office of Energy Research, U.S. Department of Energy.

\section{References}

1. H. C. Bryant, David A. Clark, Kenneth B. Butterfield, C. A. Frost, I. Sharifian, H. Tootoonchi et al., Phys. Rev. A 27, 2889 (1983).

2. F. H. Rcad, Aust. J. Phys. 35, 475 (1982).

3. P. G. Harris, H. C. Bryant, A. H. Mohagheghi, C. Y. Tang, J. B. Donahue, C. R. Quick et al., "Measurement and Reduction of Momentum Spread in the LAMPF Linac Beam" (submitted to Nuclear Instruments and Methods A). 


\section{EXPERIMENT 1127 - HIRAB \\ Multiphoton Detachment of Electrons from the $\mathrm{H}^{-}$Ion}

\author{
Univ. of New Mexico, Los \\ Alamos, St. Mary's \\ College, California State \\ Univ., Univ. of Mashad \\ (Iran), Oswego High \\ School (New York) \\ Spokesmen: C. R. Ouick, Jr. (Los \\ Alamos) and H. C. Bryant (Univ. \\ of New Alexico) \\ Participants: P. G. Harris, H. C. \\ Bryant, A. H. Mohaghoghi, \\ R. A. Reeder, C. Y. Tang, \\ J. B. Donahue, C. R. Quick, \\ Jr., D. C. Rislove, H. Sharfilan, \\ H. Tootoonchi, and T. C. Altman
}

\section{Status Report}

This experiment was granted $486.8 \mathrm{~h}$ of beam time following a recommendation of $300 \mathrm{~h}$ by the PAC, with A priority during FY 1989, to demonstrate that multiphoton-induced (MPI) electron detachment from the $\mathrm{H}^{-}$ion could be observed with the currently a vailable lasser technology. This would therefore establish the feasibility of carrying out the rescarch described in detail in LAMPF Proposal 1127. The initial experiments were carried out using a $\mathrm{CO}_{2}$ oscillator/amplifier laser system capable of delivering linearly-polarized temporallysmooth pulses at 9.4 and $10.6 \mu \mathrm{m}$, with a peak power density approximately $2 \times 10^{10} \mathrm{~W} / \mathrm{cm}^{2}$ at $0.5 \mathrm{~Hz}$. First results are encouraging, and have been described briefly in the scientific literature. ${ }^{1,2}$ Comparisons between our experimental results and theoretical models for $\mathrm{H}^{-}$multiphoton absorption already appear in the literature. ${ }^{3}$

\section{Summary of Experimental Results}

$\mathrm{H}^{-}$is easily "ionized" by the available laser pulses. To date, we have observed MPI at center-of-mass (CM) photon energies that correspond to 2-through 8-photon absorption. The preliminary scans of the MPI electron yield vs photon energy have revealed clear transitior.s (thresholds) between different orders of photon absorption ( $N=2-5$ ). Figure 1 is a plot of the measured electron yield as a function of $\mathrm{CM}$ laser photon energy observed using an $\mathrm{H}^{-}$beam at $800 \mathrm{MeV}$ with a $10.6-\mu \mathrm{m}$ laser energy of approximately $0.6 \mathrm{~J} /$ pulse. Over the photon energy range shown, a minimum of two or three photons is required to ionize $\mathrm{H}^{-}$. The transition from 2- to 3-photon absorption is clearly evident, occurring at approximately $0.375 \mathrm{eV}$. It seems most likely that the fluctuations in the data are the result of noise rather than true structure in the cross section. Reducing the laser pulse energy alters the appearance of the electron yicld vs energy plot; the $N=3$ part of the curve is suppressed relative to the $N=2$.

A careful examination of the transition region that occurs between different orders of photon absorption can be used to determine the extent to which the intense electromagnetic fields shift the ionization threshold energy $(0.754 \mathrm{eV}$ for zero field). From the data obtained so far, we have established that the $\mathrm{H}^{-}$ ionization threshold energy level shift is smaller than the peak ponderomotive potential energy created by the intense laser radiation. A more accurate, quantitative measurement of the shift will require an experiment with better statistics.

A few measurements of the MPI electron yield vs laser intensity were also carried out. The data clearly reflect the nonlinear nature of the absorption process and show signs of saturation. 


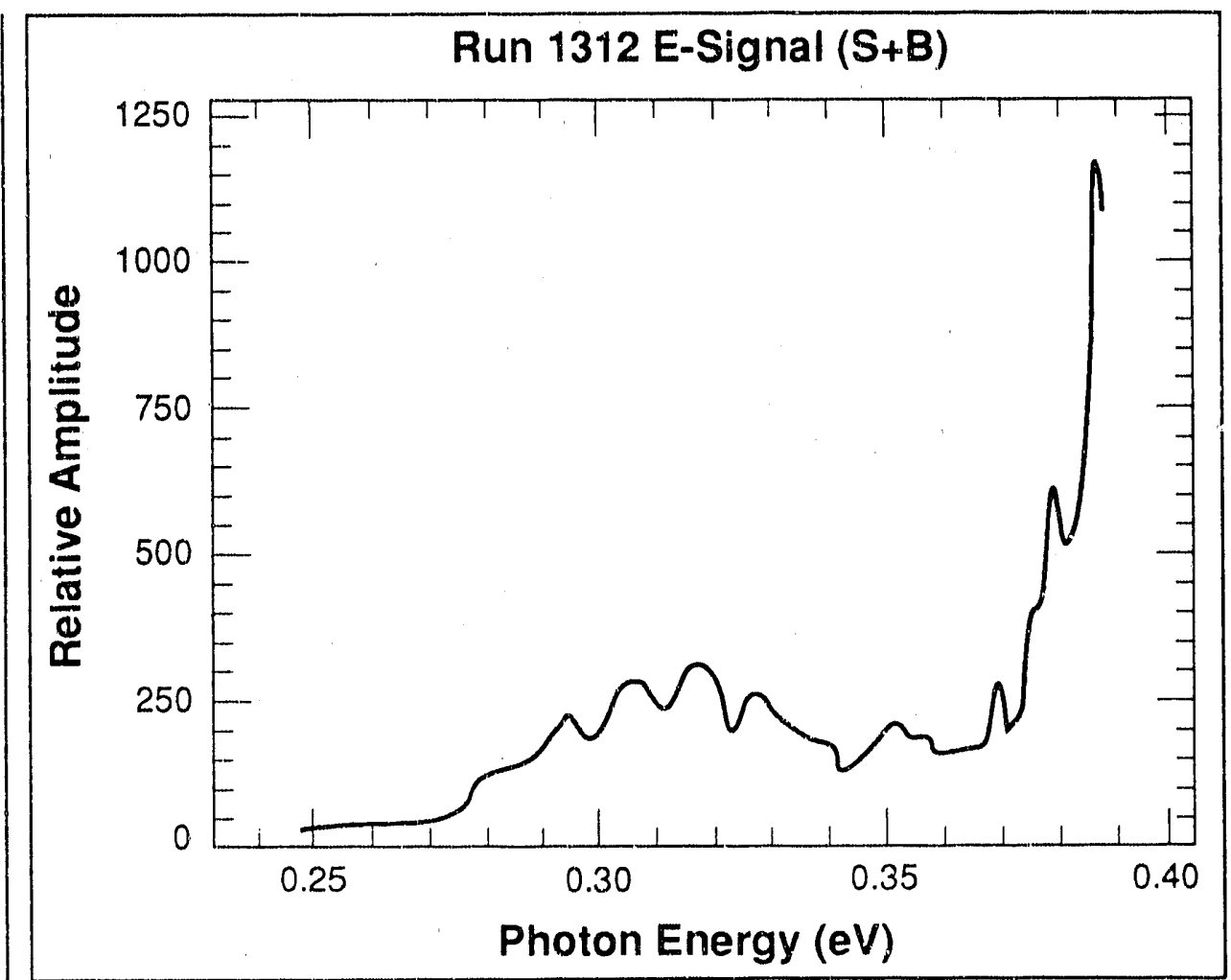

Fig. 1. Relative electron yield vs photon energy for multiphoton detachment of $\mathrm{H}^{-}$, for an energy range covering the 3-photon detachment process and the transition to the 2-photon process. The fluctuations represerit statistical uncertainties rather than true structure.

\section{Experimental Plans and Goals}

The quality of the data obtained so far has been limited primarily by the low repetition rate of the laser and by dynamic range/saturation effects in our particle detectors. (The size of the signal may vary over two orders of magnitude during a single run.) Each data point in Fig. 1 is the result of only 8-10 laser pulses. For the next series of experiments, we plan to have a laser system capable of running at $5-10 \mathrm{~Hz}$ that will greatly enhance our data-gathering capability and will also be more resistant to the effects of (beam-related) radiation that caused severe misfiring in our current system.

Our objectives for the upcoming operations period are:

1. Complete the detailed examination of the MPI cross section vs CM photon energy, laser intensity, and laser polarization (linear vs circular). This is essentially the work discussed in Phase I of Proposal 1127. 
2. Make a careful measurement of the $\mathrm{H}^{-}$ionization threshold shift vs laser intensity.

3. Test a charge-coupled device (CCD) detector array that is to be used during Phase II of our proposal, in which we wili examine the AboveThreshold-Ionization (ATI) process. Eventually, we hope the CCD array can be used to make spatially-resolved (and therefore energy-resolved) measurements of the individual electrons liberated from $\mathrm{H}^{-}$via MPI.

This experiment is supported in part by a grant from the Division of Chemical Sciences, Office of Basic Energy Sciences, Office of Energy Research, U.S. Department of Energy.

\section{References}

1. C. Y. Tang, P. G. Harris, H. C. Bryant, A. H. Mohagheghi, J. B. Donahue, C. R. Quick ct al., Phys. Rev. A 39, 6068 (1989).

2. W. W. Smith, C. Y. Tang, C. R. Quick, H. C. Bryant, P. G. Harris, A. H. Mohagheghi et al., "Spectra from Multiphoton Electron Detachment of $\mathrm{H}^{-1}$ (to be published in the Journal of the Optical Society of America B).

3. X. Mu, "Multiphoton Absorption Probabilities in Strong Laser Fields with Application to $\mathrm{H}^{-\prime \prime}$ (submitted to Physical Review A). 
EXPERIMENT 811 - LEP

\section{Study of Unnatural Parity States in Nuclei Using Low- Energy Pions}

\author{
Arizona State Univ., \\ Los Alamos, IJniv. \\ of Colorado, Univ. of \\ South Carolina, Virginia \\ Polytechnic Institute, State \\ Univ. \\ Spokesman: B. G. Ritchie (Arizona \\ State Univ.) \\ Participants: G. S. Adams, G. S. \\ Blanpied, R. L. Boudrie, J. A. \\ Escalante, C. S. Mishra, J. H. \\ Mitchell, R. J. Peterson, B. M. \\ Preedom, B. G. Ritchle, C. S. \\ Whisnant, and D. H. Wright
}

Studies of pion-nucleus reactions at energies below the delta resonance have been pursued because of the vast difference in the underlying pion-nucleon reaction dynamics between low and resonance energies. While a detailed understanding of the phenomena involved in low energy pion-nucleus scattering requires data on a varicty of nuclei and on a variety of states within those nuclei, data obtained in the past have primarily been for pion elastic scattering from nuclei with relatively simple structure because of the constraints dictated by the poor energy resolution of the devices used in those experiments.

Recently, investigations of low-energy pion-nucleus scattering have been greatly enhanced by the development of a new generation of low-energy pion spectrometers with good energy resolution. The development of these devices has provided an opportunity to study low-energy pion scattering to nuclear levels that were previously inaccessible. In a Rapid Communication, ${ }^{1}$ we reported the results of an experiment using such a device-a study of the pair of $1^{+}$ states, at $12.7(T=0)$ and $15.1(T=1) \mathrm{MeV}$, in ${ }^{12} \mathrm{C}$. Data were also taken on other states in ${ }^{12} \mathrm{C}$. Elastic scattering data were taken for both $\pi^{+}$and $\pi^{-}$during our measurements of the cross sections for the ${ }^{12} \mathrm{C} 1^{+}$doublet. Previous measurements ${ }^{2-5}$ of the elastic scattering of positive pions from ${ }^{12} \mathrm{C}$ at $50 \mathrm{MeV}$ were in significant disagreement for pion-scattering angles beyond $90^{\circ}$. Thus, the elastic-scattering data taken during this experiment are of considerable interest in providing insight into resolving this discrepancy. Studies of the inelastic excitations in ${ }^{12} \mathrm{C}$ can provide a test of the applicability of simple models of the transition densities and the effects of coupled-reaction channels. The results from previous pion inelastic-scattering experiments have presented cross sections for several of the excited states of ${ }^{12} \mathrm{C}$, but in some cases no confirmation of those results was available or the confirmation has been ambiguous.

The data for elastic positive-pion scattering from ${ }^{12} \mathrm{C}$ are shown in Fig. 1 , along with data taken previously. ${ }^{2-5}$ The data taken in this study strongly favor the measurements of Precdom et al. ${ }^{4}$ and Sobic et al. ${ }^{5}$ within the angular range studied. The disagreement between the data taken here and that of Dytman et al., ${ }^{3}$ though within the quoted normalization error for that work (15\%), is seen in all comparisons with states measured here and in that work. The discrepancy between this work and that of Johnson et al." is more serious, with disagreement in both the position of the minimum and the magnitude of the cross sections at all but the smallest angles. We conclude that the measurements reported in Refs. 4 and 5 correctly describe the cross sections for elastic scattering of positive pions from ${ }^{1 "} \mathrm{C}$ at $50 \mathrm{McV}$. With some renormalization, the data from Ref. 3 are substantially in agreement as well. The elastic differential cross sections for negative pion scattering from ${ }^{12} \mathrm{C}$ at $50 \mathrm{MeV}$ obtained in this work are shown in Fig. 2, again compared to previous data. The data obtained here are in general agreement with the data reported in Ref. 5, disagreeing by little more than the normalization errors. However, the data taken here again seriously disagree with the data of Ref. 2, particularly at large scattering angles. 


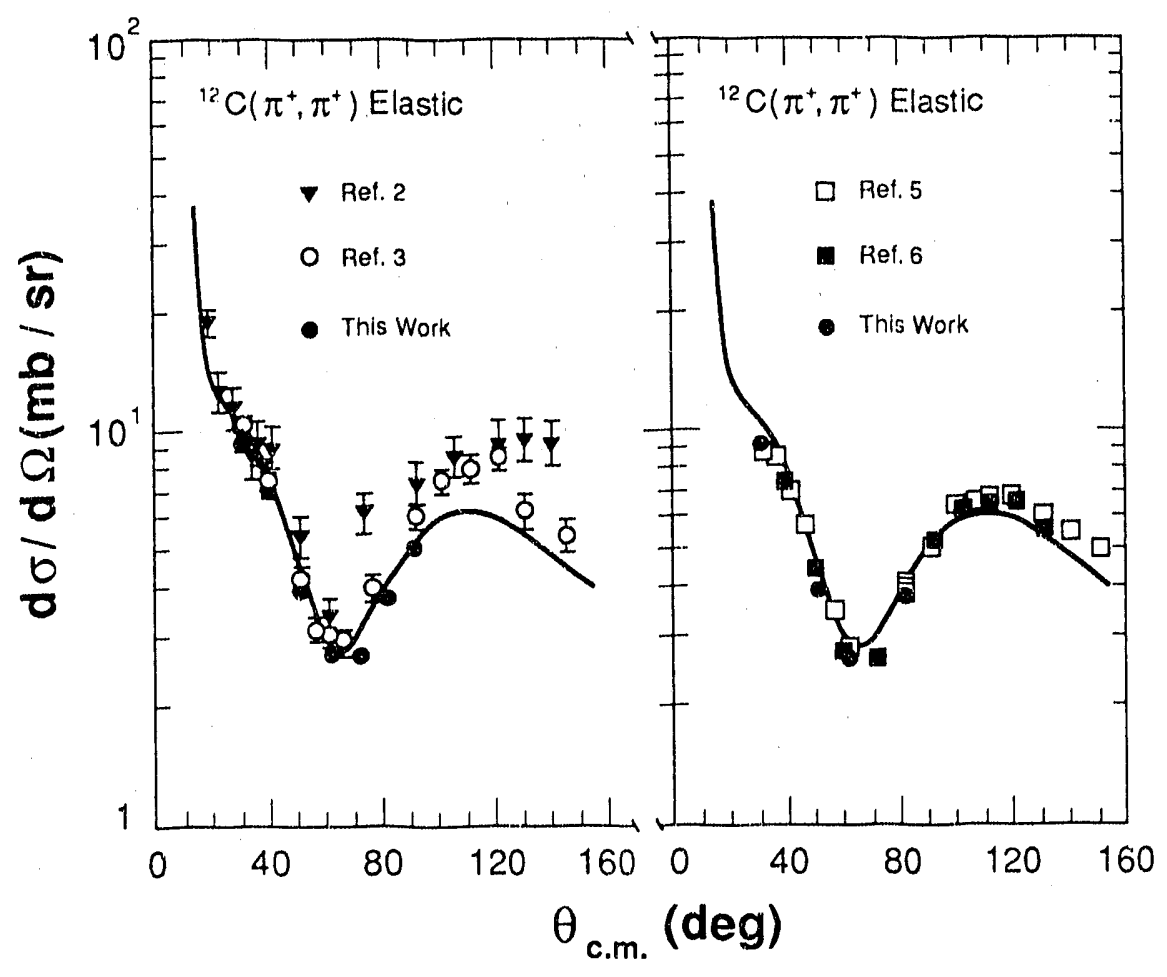

Fig. 1. Measured differential cross sections for elastic scattering of positive pions at $50 \mathrm{MeV}$ from ${ }^{12} \mathrm{C}$. Data are from this work und from Refs. 3-6. Error bars shown include statistical error only. The solid (dashed) line indicates the results of the one-step (coupled-channels) calculation described in the text.

The differential cross sections for the excitation of the first excited state of ${ }^{12} \mathrm{C}$ at $4.44 \mathrm{MoV}$ by positive pions, as determined in this study, are shown in Fig. 3, along with data reported previously in Refs. 3 and 5. Once again, the data taken here are in excellent agreement with the data of Ref. 5, and disagree with the data of Ref. 3, mainly in overall normalization by approximately the same factor as seen in the elastic angular distribution. The data obtained here for positive pion excitation of the 7.65- $\mathrm{McV}, 0_{2}^{+}$state in ${ }^{12} \mathrm{C}$ are shown in Fig. 4, along with data taken previously ${ }^{3,6}$ for that state. The data of Lee et al. ${ }^{6}$ are in excellent agreement with the data obtained in our studies. The data of Ref. 4, while in disagreement, have very large uncertainties. Data obtained here for the excitation of the $9.64 \mathrm{McV}, 3^{-}$state are displayed in Fig. 5, along with the data reported in Ref. 3. The previously reported data again appear to be in disagreement in overall normalization, but since the angular ranges in that work and this study are for the most part different, it is not unambiguously clear that the difference is significant. 


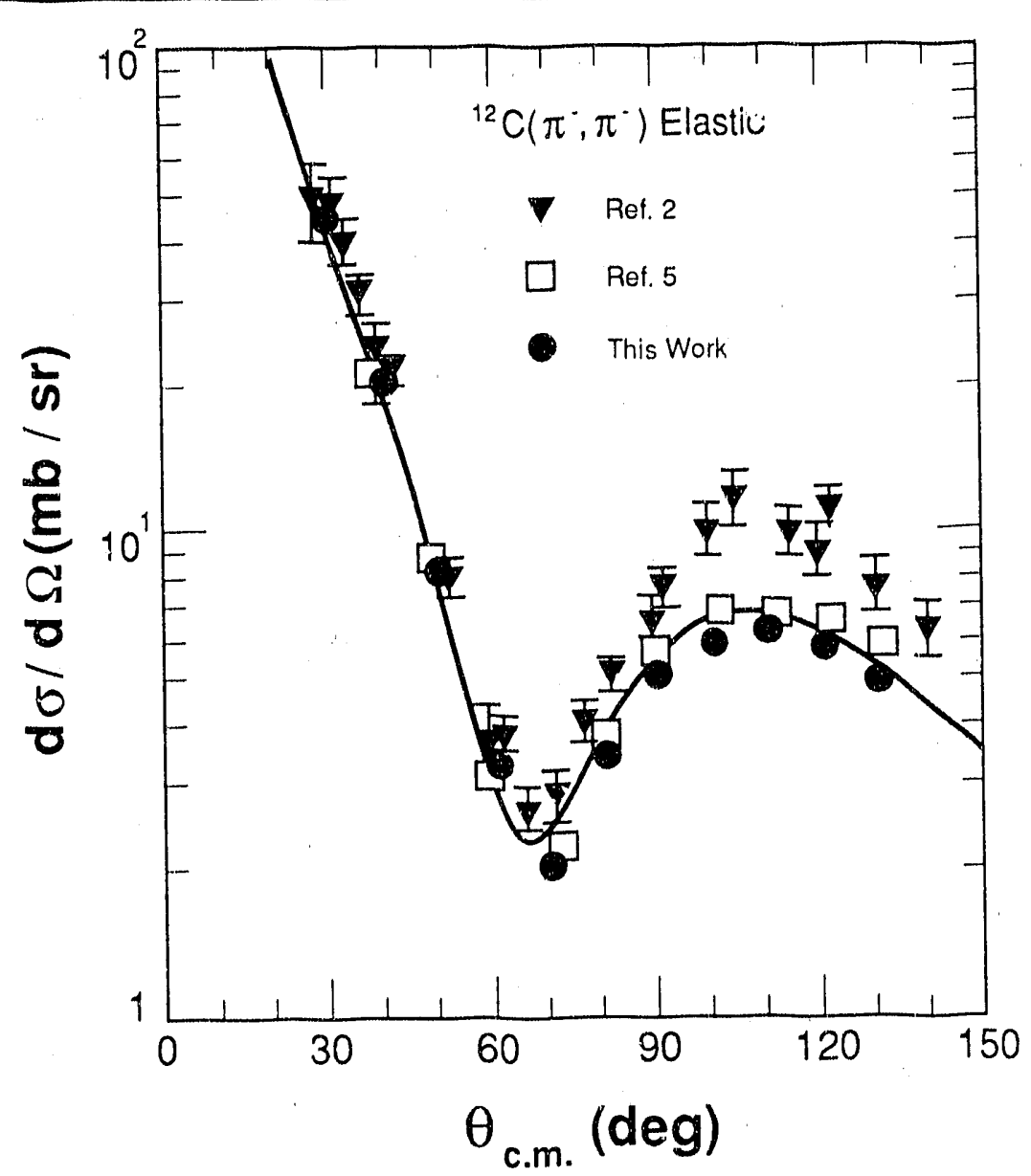

Fig. 2. Measured differential cross sections for elastic scattering of negative pions at $50 \mathrm{MeV}$ from ${ }^{12} \mathrm{C}$. Data are from this work and from Refs. 2 and 6. Error bars shown include statistical error only. The solid (dashed) line indicates the results of the one-step (coupled-channels) calculation described in the text.

The results of the measurements reported in this study primarily concern states in ${ }^{12} \mathrm{C}$, which are believed to be essentially collective isoscalar excitations. However, the excitation of the $0_{2}^{+}$state is generally not explained well with the use of simple one-step collective transition densities. To confirm these observations, predictions for the cross sections of the excited states, made using the method discussed in a systematic analysis of data obtained for pion scattering at 50 and $65 \mathrm{McV}$ by one of the participants, ${ }^{7}$ are compared with the data presented in Figs. 1-6. A full discussion of the procedure used in arriving at the predictions, discussed below, may be found in that work. Both one-step (OS) and 


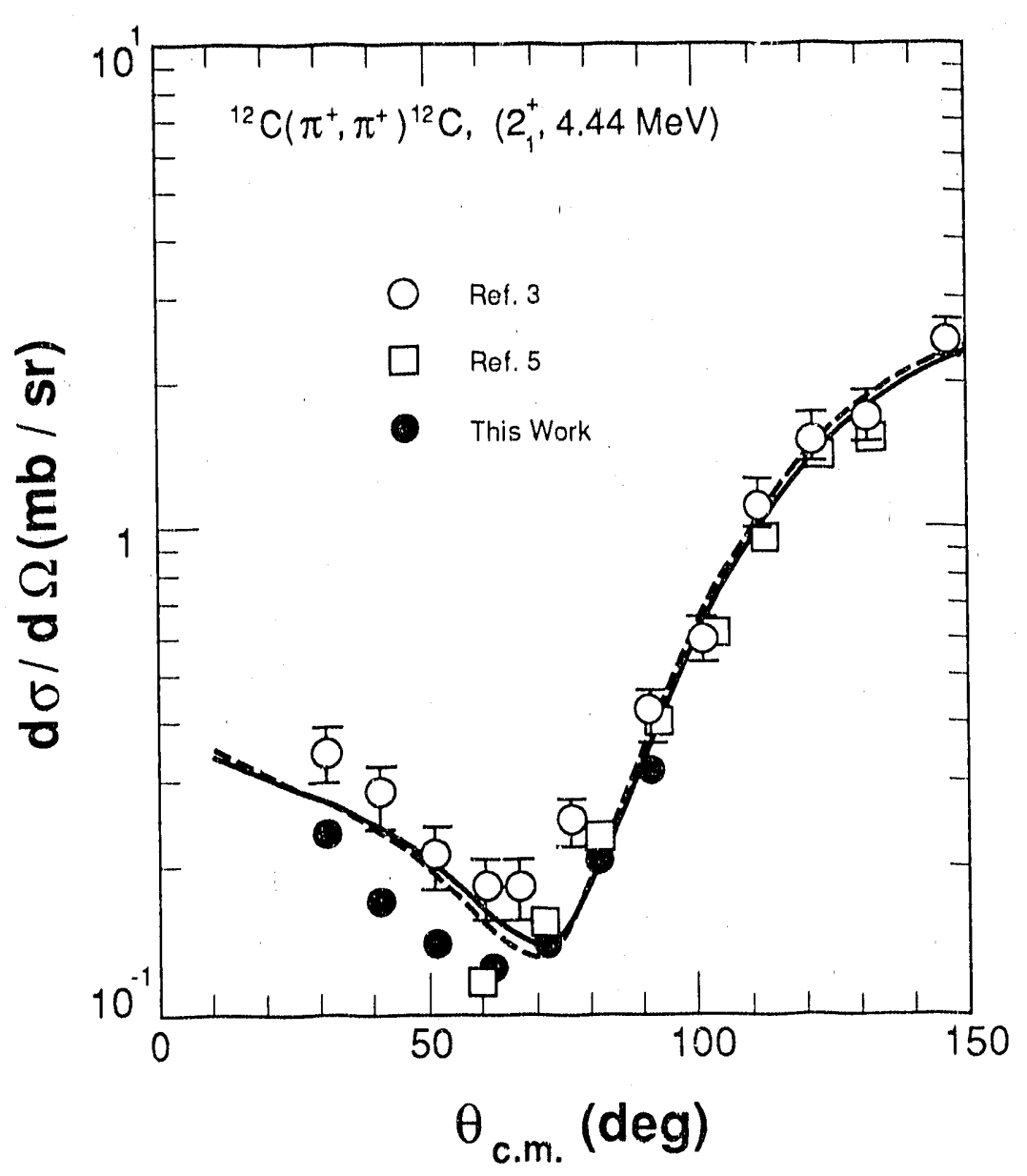

Fig. 3. Measured differential cross sections for excitation of the 4.44-MeV, $2+$ level of ${ }^{12} \mathrm{C}$ by positive pions at $50 \mathrm{MeV}$. Data are from this work and from Refs. 4 and 6. Error bars shown include statistical error only. The solid (dashed) line indicates the results of the one-step (coupled-channels) calculation described in the text.

coupled-channels $(\mathrm{CC})$ calculations were performed using a modification of the MSU potential ${ }^{*}$ by U. Wienands et al.; ${ }^{\prime 9}$ this modified potential is denoted as MSUT in Ref. 7. The CC calculation included the effects of two-step excitation of the $0_{2}^{+}$and $3^{-}$states through the first excited state, the $2^{+}$state at $4.44 \mathrm{MeV}$, with deformation lengths of $\beta_{2} R=1.24 \mathrm{fm}$ and $\beta_{3} R=0.90 \mathrm{fm}$, as found in Refs. 7 and 10 , respectively; a discussion of those deformation lengths may be found in Ref. 7. The one-step transition density to the $0_{2}^{+}$state was based on the fit to the electron-scattering data by Sparrow and Gerace. ${ }^{11}$ In the comparisons of the results of the calculations in r.igs. 1-6, the one-step results are indicated by solid lines, while the coupled-channels results are shown with broken lines. 


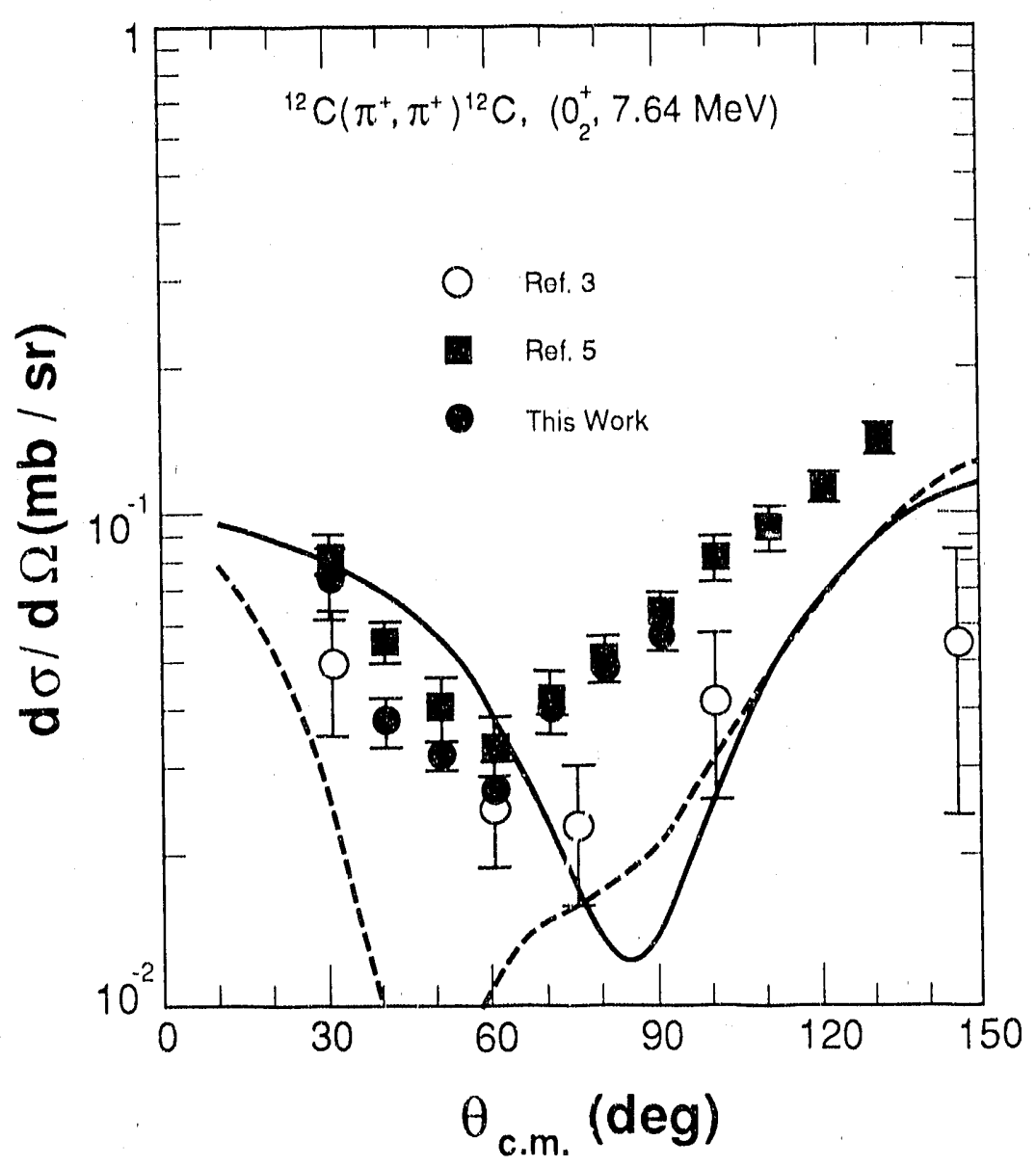

Fig. 4. Measured differential cross sections for excitation of the 7.65-MeV, (0) level of ${ }^{12} \mathrm{C}$ by $5(\mathrm{M}-\mathrm{MeV}$ positive pions. Data are from this work and from Refs. 4 and 6. Error bars shown include statistical error only. The solid (dashed) line indicates the results of the one-step (coupled-channels) calculation described in the text.

The resulting calculations are compared with the data for $\pi^{+}$elastic scattering from this and previous studies in Fig. 1, with a similar comparison for the $\pi^{-}$elastic-scattering data in Fig. 2. In both figures, it is seen that the calculations give an excellent description of the data reported in this work, and that the OS and CC calculations are virtually indistinguishable. Similarly, the results of the calculations are compared to the angular distributions for $\pi^{+}$scattering to the $2^{+}, 4.44-\mathrm{MeV}$ state in Fig. 3, and again it is seen that the results of the two calculations are nearly indistinguishable and both describe the data very well. 


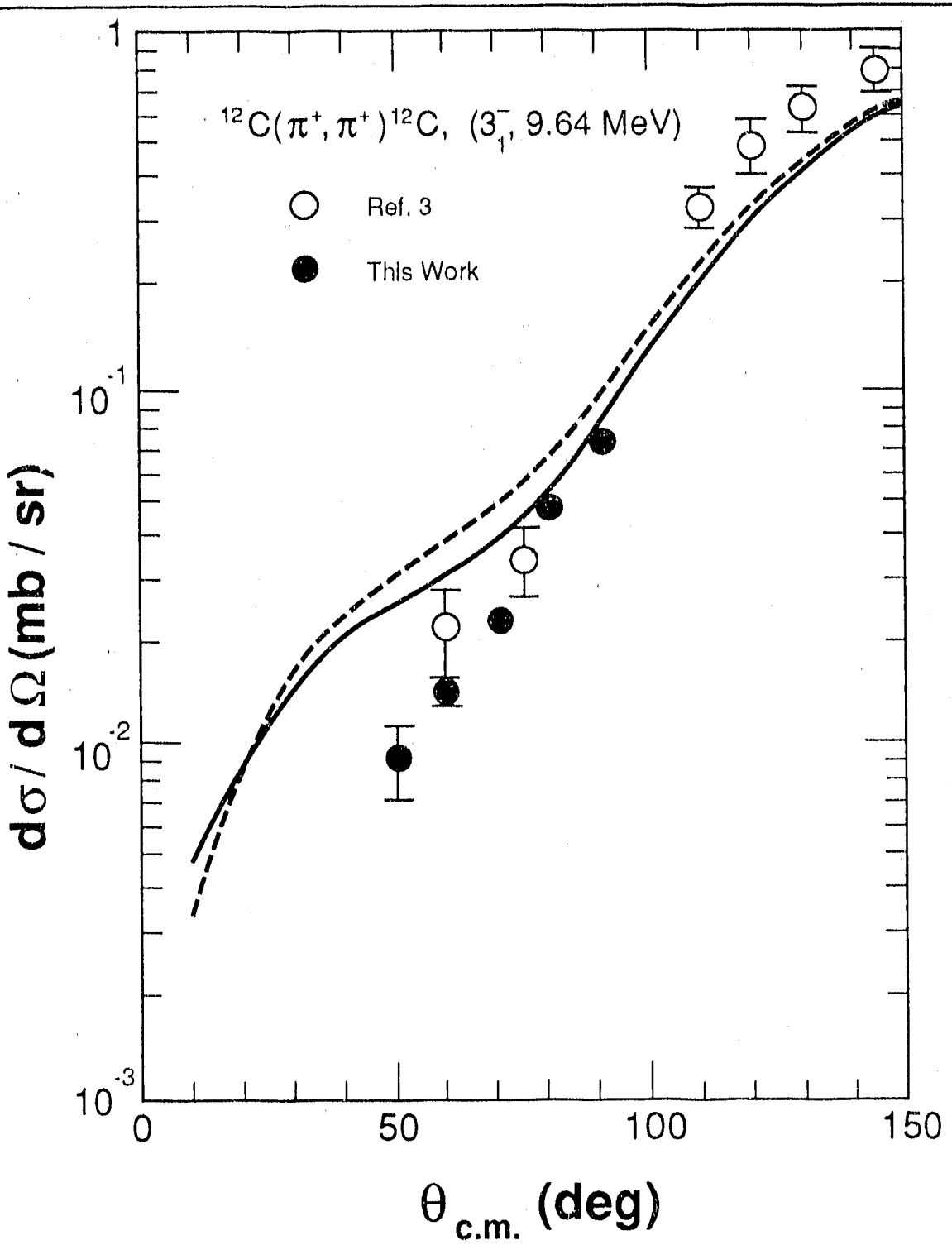

Fig. 5. Measured differential cross sections for excitation of the 9.64-MeV, $3^{-}$level of ${ }^{12} \mathrm{C}$ by 50-MeV positive pions. Data are from this work and from Ref. 4. Error bars shown include statistical error only. The solid (dashed) line indicates the results of the one-step (coupled-channels) calculation described in the text.

Shown in Fig. 5 are predictions for the excitation of the $9.64-\mathrm{MeV} \mathrm{3-}$ state by positive pions. Qualitatively, both calculations describe the data obtained here and in Ref. 3, though the data appear to require a steeper angular dependence than either calculation provides. Since the calculations performed used a 
spherical vibrational model for the nucleus, better agreement might be obtained by removing that assumption. Additionally, only couplings where $m=0$ were available in the code used; other contributions from terms with nonzero $\mathrm{m}$ might provide a different slope.

Overall, the strong agreement between the OS and CC calculations and the agreement of the results of the OS calculation with the data presented in Figs. 1, 2,3 , and 5 provide strong confirmation that the dominant component in each of the transition matrix elements to the ground, first excited, and third excited states are those given by a simple one-step derivative-collective transition density with little evidence for coupled-channels effects.

The results obtained for the $0_{2}^{+-}$state are shown in Fig. 4. As may be seen in the figure, the data for the $0_{2}^{+}$data are not well described by the OS calculations. Though Jennings and Takacsy ${ }^{12}$ attributed this failure to the LorentzLorenz-Ericson-Ericson effect, Ref. 7 argues that this lack of agreement can be interpreted as a manifestation of coupled-channels effects in the excitation of that state, particularly coupling through the first excited state. These effects are very pronounced and proper treatment is crucial in achieving agreement between the calculation and the data obtained here. The CC calculation, though disagreeing in overall magnitude, is qualitatively better in its description of the data. A thorough discussion of the ramifications of this comparison may be found in Ref. 7.

\section{References}

1. B. G. Ritchie, J. A. Escalante, G. S. Adams, M. Al-Solami, N. Lasheen, G. Pignault et al., Phys. Rev. C 37, 1347 (1987).

2. R. R. Johnson, T. G. Masterson, K. L. Erdman, A. W. Thomas, and R. H. Landau, Nucl. Phys. A 296, 444 (1978). Though the data in this reference were obtained at $49.5 \mathrm{MeV}$, the difference in pion energy is small enough to warrant comparison with the other work at $50 \mathrm{MeV}$.

3. S. A. Dytman, J. F. Amann, P. D. Barnes, J. N. Craig, K. G. R. Doss, R. A. Eisenstein et al., Phys. Rev. C 19, 971 (1979).

4. B. M. Preedom, S. H. Dam, C. W. Darden III, R. D. Edge, I. J. Malbrough, T. Marks et al., Phys. Rev. C 23, 1134 (1981).

5. R. J. Sobic, T. E. Drakc, K. L. Erdman, R. R. Johnson, H. W. Roser, R. Tacik et al., Phys. Rev. C 30, 1612 (1984).

6. L. Lee, T. E. Drake, L. Buchmann, A. Galindo-Uribarri, R. Schubank, R. J. Sobie ct al., Phys. Lett. 174B, 147 (1986).

7. C. S. Whisnant, Phys. Rev. C 40, 1741 (1989).

8. J. A. Carr, H. McManus, and K. Stricker-Bauer, Phys. Rev. C 25, 952 (1982). 
9. U. Wienands, N. Heasey, B. M. Barnett, F. M. Rozon, H. W. Roser, A. Altman et al., Phys. Rev. C 35, 708 (1987).

10. A. Ingemarsson, O. Jonsson, and A. Hallgren, Nucl. Phys. A 319, 377 (1979).

11. D. A. Sparrow and W. J. Gerace, Phys. Rev. C 29, 949 (1984).

12. B. K. Jennings and N. de Takacsy, Phys. Lett. 124B, 302 (1983). 
EXPERIMENT 948 - - LEP

\section{The Role of Quasi- Deuterons in Pion Absorption on ${ }^{6} \mathrm{Li}$}

\author{
Arizona State Univ., New \\ Mexico State Univ., Univ. \\ of South Carolina, Univ. \\ of Virginia \\ Spokesman: B. G. Aitchle (Arizona \\ State Univ.) \\ Partlcipants: N. S. Chant, J. R. \\ Comlort, J. A. Escalante, R. \\ Glannell, G. S. Kyle, D. Mack, \\ B. M. Preedom, M. Rawool, \\ B. G. Flichle, P. G. Roos, D. \\ Rothenberger, J. D. SIIK, and \\ J. R. Tinsley
}

The intense experimental and theoretical efforts to understand the fundamental pion-absorption process $\pi^{+}+d \rightarrow p+p$ have resulted in a sophisticated and generally successful description of the basic phenomena associated with that reaction. One path for extending this understanding to heavier nuclei is to study systems where the nucleus can be modeled as a cluster, consisting of a deuteronlike pair of nucleons and some residual core, attempting to relate absorption on this bound quasi-deuteron to the free process.

${ }^{6} \mathrm{Li}$ can be modeled as being made up of a deuteron orbiting an alpha with relative angular momentum zero. Such a picture suggests that the nucleus "Li would be a very productive "laboratory" for studying pion absorption on deuteron-like structures within the nucleus. Absorption on the ground state would be nearly identical to the process $\pi^{+}+d \rightarrow p+p$; absorption on excited states would give insight into the process on nucleon pairs differing from deuteron-like structures. Both could be studied in a cluster-model formalism, with the ground-state process providing information on the entrance- and exitchannel distorted waves for the excited states. Studies at a number of energies below, at, and above resonance would supply additional information on the effects of an intermediate state delta in absorption on different pairs of nucleons. Two experiments have been developed to provide cross sections pertinent to these ideas. Experiment 948 at LAMPF studied the ${ }^{6} \mathrm{Li}\left(\pi^{+}, 2 p\right)$ reaction below resonance; an experiment at Paul Scherrer Institute (PSI) provided data at and above resonance.

In Exp, 948, data were obtained at 30,50, 80, and $115 \mathrm{MeV}$ for the reaction ${ }^{6} \mathrm{Li}\left(\pi^{+}, 2 p\right)$. The rolled metal " $\mathrm{Li}$ target was contained inside a scattering chamber with mylar windows on its perimeter. Reaction products were detected with six NaI-plastic scintillator detector telescopes. The detectors provided an overall missing-mass resolution of approximately $4 \mathrm{MeV}$. Relative normalization of the cross sections was obtained using pairs of scintillators to detect decay muons from the inciderit pion beam and the normal production beam current monitors. Absolute normalization of the cross sections was made by measuring the cross sections for the process $\pi^{+}+d-p+p$ at each energy, giving an overall normalization uncertainty of less than $13 \%$ in all cases.

Cuts on the missing mass in the final state were made at:

1. -20 to $10 \mathrm{MeV}$, to provide ground-state absorption cross sections;

2. 10 to $35 \mathrm{MeV}$, to yield cross sections for absorption on the alpha;

3. 35 to $140 \mathrm{MeV}$, to determine cross sections for highly excited states of the system; and

4. greater than $140 \mathrm{MeV}$, to give cross sections where at least a missingmass equivalent to a pion mass was present.

These cuts were chosen to provide overlap with similar cuts made on previous data on ${ }^{6} \mathrm{Li}$ pion absorption. 
The ground-state cuts reveal a sharp peak centered at the angles appropriate for $\pi^{+}+d \rightarrow p+p$, as expected. As additional missing mass is dictated by the cuts placed on the data, the cross sections remain peaked at this angle, but the angular distribution spreads markedly. Such broadening may be attributed to results of final-state interactions on the outgoing protons, following a quasideuteron-absorption event, or as evidence of more complex processes. Analyses of these data in both a cluster-model formalism and by modeling the process as proceeding via only quasi-deuteron absorption in an intranuclear cascade calculation are underway. Data from the measurements made at higher energies at PSI will be incorporated. 
EXPERIMENT 975 - L.EP

\section{Pion-Nucleus Single Charge Exchange at Threshold Energies}

\author{
Florida State Univ., Los \\ Alamos, George Mason \\ Univ., College of William \\ \& Mary, Virginia State \\ Univ.
}

\section{Spokesman: L. C. Liu (Los Alamos)}

Partlcipants: R. Nair, H. S. Plendl, E. Gavathas, R. J. Estep, B. J. Dropesky, J. D. Bowmsn, J. N. Knudson, L. C. Liu, J. B. Lieb, J. McKenzie, H. O. Funsten, and C. E. Stronach
The stopped $\pi^{-}$-induced nuclear single charge exchange (SCX) can provide information on isovector pion-nucleon interactions in nuclear media at threshold energies-information that can be used to set stringent constraints on the isovector $\pi N$ amplitude used in various low-energy pion-nucleus optical models. In the threshold-energy region, the use of stopped $\pi^{-}$-induced SCX reactions is preferred over the use of pionic atom experiments in the determination of the effective $\pi N$ isovector interaction. This is because this interaction appears as the leading-order interaction in SCX reactions, while it plays only a secondary role in pionic atom formation.

Prior to our measurements, there were only two exploratory experimental studies of nuclear $\left(\pi^{-}, \pi^{0}\right)$ reactions with stopped pions. In the first experiment, ${ }^{1}$ the nuclei $\mathrm{Li}, \mathrm{Bc}, \mathrm{C}, \mathrm{Al}, \mathrm{Ti}$, and $\mathrm{Pb}$ were used as the targets. The two $\gamma^{\prime} \mathrm{s}$ from the $\pi^{0}$ decay were detected with two Cerenkov absorption spectrometers. An upper limit for the branching ratio (BR) of $6 \times 10^{-5}$ was determined for all targets. In the second experiment, ${ }^{2}$ two large steel- and lead-shielded NaI detectors were employed. The $\mathrm{BR}$ for $\mathrm{Li}, \mathrm{C}, \mathrm{Al}, \mathrm{Ti}, \mathrm{Cu}, \mathrm{Nb}$, and $\mathrm{Pb}$ were determined with poor statistics. The energy resolution in both previous experiments was $\geq 10 \mathrm{MeV}$. Clearly, more definitive rneasurements were needed.

We used a $50-\mathrm{MeV} \pi^{-}$beam from the LAMPF LEP channel, which was slowed down to $\sim 4 \mathrm{MeV}$ is. a graphite degrader so that the pions came to a complete stop in the target. The beam was composed of $40 \% \pi^{-}$and $60 \% e^{-}$and a negligible amount of $\mu^{-}$. We determined the pion flux by means of activation technique and monitored the beam by using lead-shielded scintillation counters. The two $67.5-\mathrm{MeV} \gamma$ ray from the decay of $\pi^{0}$ were detected in coincidence in the two arms of the LAMrF $\pi^{0}$ spectrometer. The energy resolution was about $1.3 \mathrm{MeV}$ FWHM and the angular resolution was about $3.4^{\circ}$ at a target-to-firstconverter distance of $0.5 \mathrm{~m}$. This short distance was chosen to maximize the solid angle. The opening angle between the two spectrometer arms was set at $162^{\circ}$ as required by the energies of the decaying $\pi^{0}$ in the lab system; these energies varied between 1.33 and $3.66 \mathrm{MeV}$, depending on the $Q$-values of the $\left(\pi^{-}, \pi^{0}\right)$ reactions on the target nuclei.

The targets ${ }^{31} \mathrm{P}$, nat $\mathrm{S}\left(95 \%{ }^{32} \mathrm{~S}\right)$, and ${ }^{45} \mathrm{Sc}$ were chosen for the expected relatively high $B R$ arising from the good overlap between the nuclear wave functions of the initial and final nuclei. The targets ${ }^{2} \mathrm{Al}$ and "nat In were also chosen, for which we had anticipated a very small $B R$ berause of the mismatch of the initial and final nuclear wave functions. Two other drgets were also employed for the purpose of checking our measurements. The first of such targets was ${ }^{1} \mathrm{H}$ in the form of $\mathrm{CH}_{2}$, for which the BR had been determined previously. ${ }^{3-5}$ The second target was ${ }^{12} \mathrm{C}$, for which the reaction is forbidden by energy conservation. Our results are summarized in Table 1.

We have not observed $\mathrm{SCX}$ on ${ }^{115} \mathrm{in},{ }^{27} \mathrm{Al}$, and ${ }^{12} \mathrm{C}$, as anticipated. We

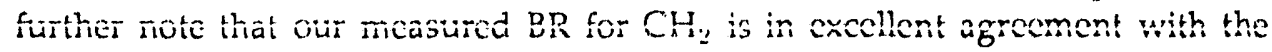
value $(7.83 \pm 1.17) \times 10^{-3}$ deduced from the measured Panofsky ratio ${ }^{6}$ and the measured probability of $\pi^{-}$capture on $\mathrm{CH}_{2}$ (Refs. 3-5). 
Table I. The Branching Ratio for SCX of the Various Targets.

\begin{tabular}{cc}
\hline Target & Branching Ratio \\
\hline $\mathrm{CH}_{2}$ & $(7.53 \pm 0.42) \times 10^{-3}$ \\
${ }^{31} \mathrm{P}$ & $(2.79 \pm 0.52) \times 10^{-5}$ \\
${ }^{n a t} \mathrm{~S}$ & $(1.16 \pm 0.26) \times 10^{-4}$ \\
${ }^{45} \mathrm{Sc}$ & $(3.27 \pm 1.17) \times 10^{-5}$ \\
\hline
\end{tabular}

In suminary, we have made the first high-resolution measurement of pionnucleus SCX at threshold energies. Our data should be of value to the development of microscopic pion reaction theories for very low energies.

\section{References}

1. V. I. Petrukhin and Yu. D. Prokoshkin, Nucl. Phys. 54, 414 (1964).

2. B. Bassaleck, F. Corriveau, M. D. Hasiroff, T. Marks, D. F. Measday, J.-M. Poutissou, and M. Salomon, Nucl. Phys. A 362, 445 (1981).

3. M. Chabre, P. Depommier, J. Heintze, and V. Soergel, Phys. Lett. 5, 67 (1963).

4. D. Bartlett, S. Devons, S. L. Meyer, and J. L. Rosen, Phys. Rev. B 136, 1452 (1964).

5. A. F. Dunaitsev, V. I. Petrukhin, and Yu. D. Prokoshkin, Nuovo Cimento 34, 521 (1964).

6. R. MacDonald, D. S. Beder, D. Berghofer, M. D. Hasinoff, D. F. Measday, M. Salomon, J. Spuller, T. Suzuki, J. M. Poutissou, R. Poutissou, P. Depommier, and J. K. P. Lee, Phys. Rev. Lett. 38, 746 (1977). 
EXPERIMENT 1023 - LEP

\section{Analyzing Power Measurements for the $\left(\pi^{+}, \pi^{0}\right)$ Reaction on a Polarized ${ }^{13} \mathrm{C}$ Target}

\author{
Arizona Siate Univ., New \\ Mexico State Univ., \\ Univ. of Minnesota, Los \\ Alamos, Univ. of Texas, \\ Univ. of Colorado, Univ. \\ of Maryland \\ Spokesmen: J. R. Comion (Arkona \\ State Univ.) and G. Kyle (New \\ Mexico State Univ.) \\ Participants: T. Avercit, J. Comfort, \\ J. DeKorse, B. Franklin, J. \\ Görgen, B. Ritchie, J. Tinsley, \\ R. Alarcon, B. Berman, G. $R$. \\ Burleson, K. Cranstor, A. Klein, \\ C. Kyle, B. Brinkmoeller, $D$. \\ Dehnhard, Yi.Fen Yen, J. A. \\ Faucett, J. Jarmer, S. Penttile \\ N. Tanaka, G. W. Hoffmann, $M$. \\ Purcell, S. Hoibraten, H. Beuer, \\ M. A. Khandaker, D. L. Naples, \\ P. G. Roos, and D. Zhang
}

A new approach to the study of the pion-nucleon isovector spin-flip interaction in nuclei was investigated in a major experiment during the summer of 1989. This experiment involved the measurement of the polarization asymmetry of the $\left(\pi^{+}, \pi^{0}\right)$ reaction on a polarized ${ }^{13} \mathrm{C}$ target at a beam energy of $165 \mathrm{MeV}$. It can be readily shown that such an asymmetry is proportional to the interference between the spin-dependent and spin-independent amplitudes of the pion-nucicon interaction. The experiment will thus provide information on the interference phase in the nuclear medium for the first time.

The target consisted of frozen beads, approximately $12 \mathrm{~mm}$ in diameter, of ethylene glycol $\left[\mathrm{OH}-\left(\mathrm{CH}_{2}\right)_{2}-\mathrm{OH}\right]$ that were placed in a ${ }^{3} \mathrm{He}$ bath inside a target cavity within a ${ }^{3} \mathrm{He}$ refrigerator. The target materials were placed in a uniform magnetic field of about 2.5 Tesla, and microwaves of frequencies near $70 \mathrm{GHz}$ were applied to the cavity. Hyperfine interactions between the electrons and the nuclei induced ${ }^{13} \mathrm{C}$ polarizations of about $26 \%$.

The ${ }^{13} \mathrm{C}$ spin parity of $1 / 2^{-}$keeps the number of spin-dependent observables to a minimum and theretore represents a good starting point for exploring pion spin physics. The IAS transition leads to the ground state of ${ }^{13} \mathrm{~N}$, which minimizes backgrounds, and the ${ }^{13} \mathrm{C}\left(\pi^{+}, \pi^{0}\right)$ cross sections have been measured at $165 \mathrm{MeV} .^{1}$ Polarization asymmetries have been predicted from two separate reaction models,, .3 and range in vaiue up to as much as 0.60 . Measurements were made over the angular range between roughly 20 $60^{\circ}$.

Since the target contained hydrogen that was polarized to approximately $80 \%$, data were also taken for the $\pi^{-} p-\pi^{0} n$ reaction to test the ability of the system for measuring asymmetries. Since there are no measurements of the polarization asymmetries for this reaction at energies below $192 \mathrm{MeV},{ }^{4}$ our data are thus new contributions to the data base on $\pi N$ scattering. The data have been analyzed and results are shown in Fig. 1 in comparison with predictions from the phase-shift code SAID. ${ }^{5}$

\section{Refer ances}

1. A. Doron, J. Alster, A. Erell, M. A. Moirester, R. A. Anderson, Phys. Rev. C26, 189 (1982).

2. D. Erns!, in Workshop on Physics with Polarized Nuclear Targets, J. J. Jarmer, Ed., Los Alarnos National Laboratory report LA-10550-C (1986).

3. P. Siegel, in Workshop on Physics with Polarized Nuclear Targets, J. J. Jarmer, Ed., Los Alarios National Laboratory report LA-10550-C (1986).

4 G. Kim, Ph.D. thesis, UCLA, Los Alamos National Laboratory report LA11273-T (1988).

5 R. A. Arndt, computer program SAID.

6 M. E. Sevior, A. Feitham, F'. Weber, G. K. Smith, D. K. Giii, D. Heaiey, Phys. Rev. C 40, 2780 (1989). 


\section{RESEARCH}

Nuclear and Particle Physics

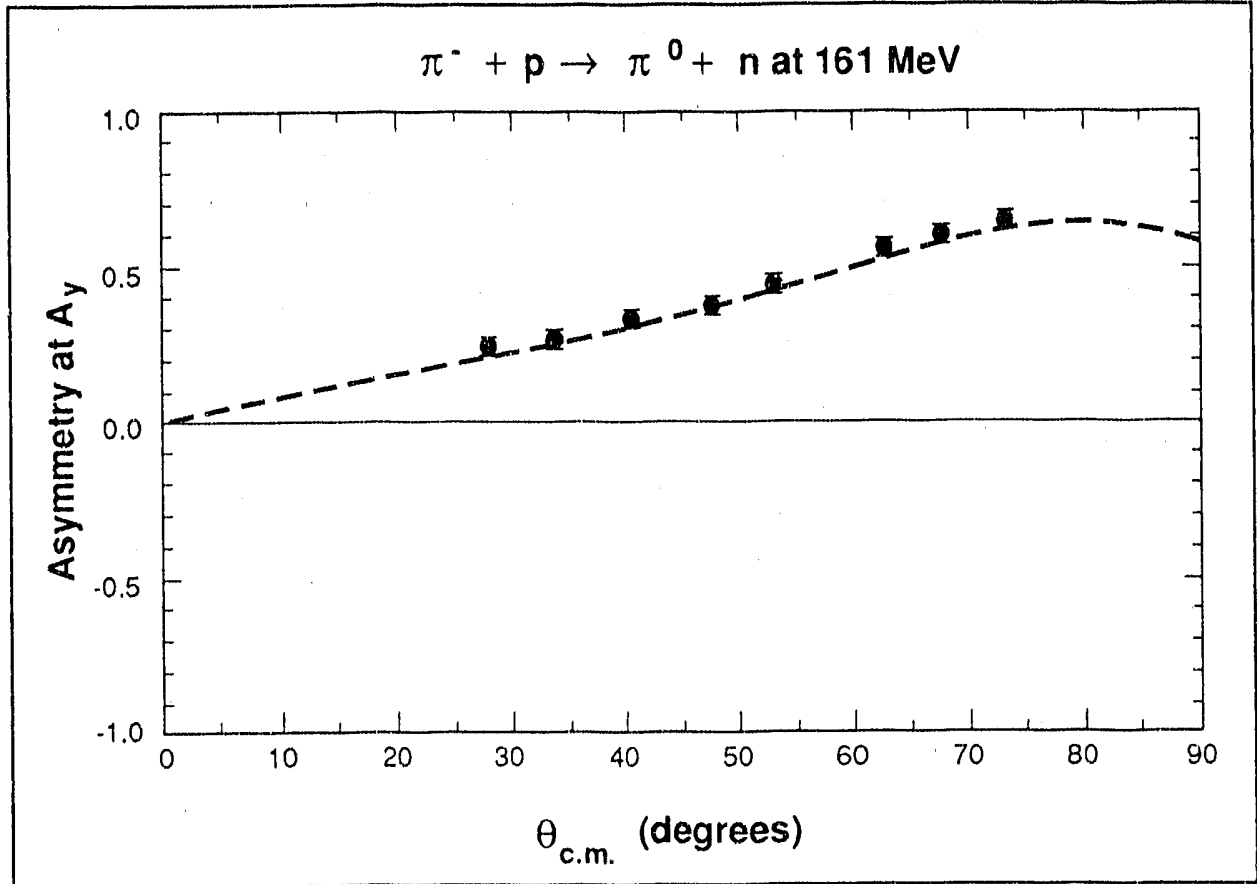

Fig. 1. Polarization asymmetry for the $\pi^{-} p \rightarrow \pi^{0} n$ reaction at an average pion beam energy of $161 \mathrm{MeV}$ in a polarized hydrogen target. The curve is the result of a calculation in which the phases have been obtained by fits to a large data base, including recent measurements of $\pi N$ elastic polarization asymmetries (Ref. 6). 
EXPERIMENT 1025 - LEP

\section{Pion Elastic Scattering from Polarized ${ }^{13} \mathrm{C}$}

New Mexico State Univ., Unit. of Minnesota, Arizona State Univ., Los Alamos, Univ. of Texas, Univ. of Pennsylvania, Univ. of Colorado, UIniv. of Maryland

Spokesmen: G. R. Burleson (New Mexico State Univ.) and $D$. Dehnhand (Univ. of Minnesota)

Participants: B. Berman, G. R. Burleson, K. Cranston, A. Klein, G. Kyle, B. Brinkmoelier, D. Dehnhard, S. M Sierbenz, YiFen Yen, Yivu Yu, T. Averett, J. Comfont, J. Goergen, B. Richtie, J. Tinsley, R. Alarcon, G. W. Hoffmann, $K$. Johnson, $M$. Purcell, H. Ward, A. Williams, J. A. Faucett, S. J. Greene, J. Jarmer, J. McGill, C. L. Morris, S. Penttilä, N. Tanaks, E. Insko, R. Nie, J. M. O'Donnell, D. $S$ mith, S. Hoibraten, and $M$ Khandaker
During 1989, Exps. 1023 and 1025 were done at LAMPF with pion beams on polarized nuclear targets. These experiments promise to reveal new features of the pion-nucleus interaction. In this report, we discuss primarily Exp. 1025.

The pion-nucleon interaction, and therefore the pion-nucleus interaction, has both spin-independent and spin-dependent parts. The latter lead to spin excitations of nuclei in pion-nucleus scattering. However, usually the amplitudes of the spin excitations are small compared to the amplitudes which do not involve spin. Measurements of the right-left asymmetry determine the interference term between the spin-dependent and spin-independent scattering amplitudes and are therefore more sensitive to the small spin-dependent terms than simple cross section measurements which depend only on the squares of the amplitudes. Present pion-nucleus interaction models predict vastly different asymmetries but very similar differential cross sections. Thus, the asymmetry measurements should provide a stringent test of pion-nucleus interaction models. For example, one model that includes the interaction of the $\Delta(1232)$ isobar with the nucleus, predicts a strong dependence of the asymmetry on the strength of the $\Delta$-nucleus spin-orbit potential.

The experiments were carried out in the LEP channel at LAMPF. A polarized ${ }^{13} \mathrm{C}$ target was used which had been developed by the LAMPF polarized target group in collaboration with the University of Texas. The "right-left" asymmetry was measured in these experiments by scattering from nuclei which are polarized either "up" or "down" with respect to the reaction plane. In Exp. 1023 (J. Comfort and G. Kyle, spokesmen), the asymmetry for $\left(\pi^{+}, \pi^{0}\right)$ single-charge exchange scattering was measured at $164 \mathrm{MeV}$ with the $\pi^{0}$ spectrometer system. In Exp. 1025, the asymmetry was obtained for $\pi^{+}$and $\pi^{-}$elastic scattering over a range of energies and angles in the region of the $\Delta$ resonance using the Large Acceptance Spectrometer (LAS). In Exp. 1025, the energy resolution was about $3 \mathrm{MeV}$, which was adequate for elastic scattering. Both experiments used the same polarized target setup, but in Exp. 1025 the target material was butanol $\left(\mathrm{C}_{4} \mathrm{H}_{10} \mathrm{O}\right)$, which gave a smaller background from oxygen than ethylene glycol $\left(\mathrm{C}_{2} \mathrm{O}_{2} \mathrm{H}_{6}\right)$, the material used for Exp. 1023. The ethylene glycol gave no major background in Exp. 1023. The material was made in the form of frozen beads kept in a liquid ${ }^{3} \mathrm{He}$ environment at a temperature of about $0.5 \mathrm{~K}$. The carbon was enriched to $99 \%$ in ${ }^{13} \mathrm{C}$. Polarizations for ${ }^{13} \mathrm{C}$ and ${ }^{1} \mathrm{H}$ of up to $28 \%$ and $80 \%$, respectively, were achieved for both target materials in a magnetic field of $2.5 \mathrm{~T}$. The target polarizations were determined with a Nuclear Magnetic Resonance (NMR) and, for ${ }^{1} \mathrm{H}$, were found to be in good agreement with values expected from the asymmetries for $\pi^{+} p$ scattering, which were measured at 130 and $226 \mathrm{MeV}$. The same was found to be true for the ${ }^{1} \mathrm{H}\left(\pi^{-}, \pi^{0} n\right)$ reaction measured in Exp. 1023.

In Exp. 1025, measurements were made of the asymmetries for $\pi^{ \pm}$scattering from ${ }^{13} \mathrm{C}$ at $130 \mathrm{MeV}$ between $35^{\circ}$ and $125^{\circ}$ and at $226 \mathrm{MeV}$ between $40^{\circ}$ and $80^{\circ}$ (which covered about the same region of momentum transfer as at $130 \mathrm{MeV}$ ). Áculitiond nledsulenients were made for $\pi^{-}$scattering at a momentum transfer, 
corresponding to the region of a minimum in the scattering cross section (where the asymmetry is expected to be a maximum) for energies between 114 and $180 \mathrm{MeV}$. Thus far, the data at $130 \mathrm{MeV}$ for $\pi^{-}$on ${ }^{13} \mathrm{C}$ have been replayed and preliminary asymmetries have been extracted (Fig. 1). The asymmetries are very small but not zero at this energy. On-line results at the other energies also indicate very small asymmetries.

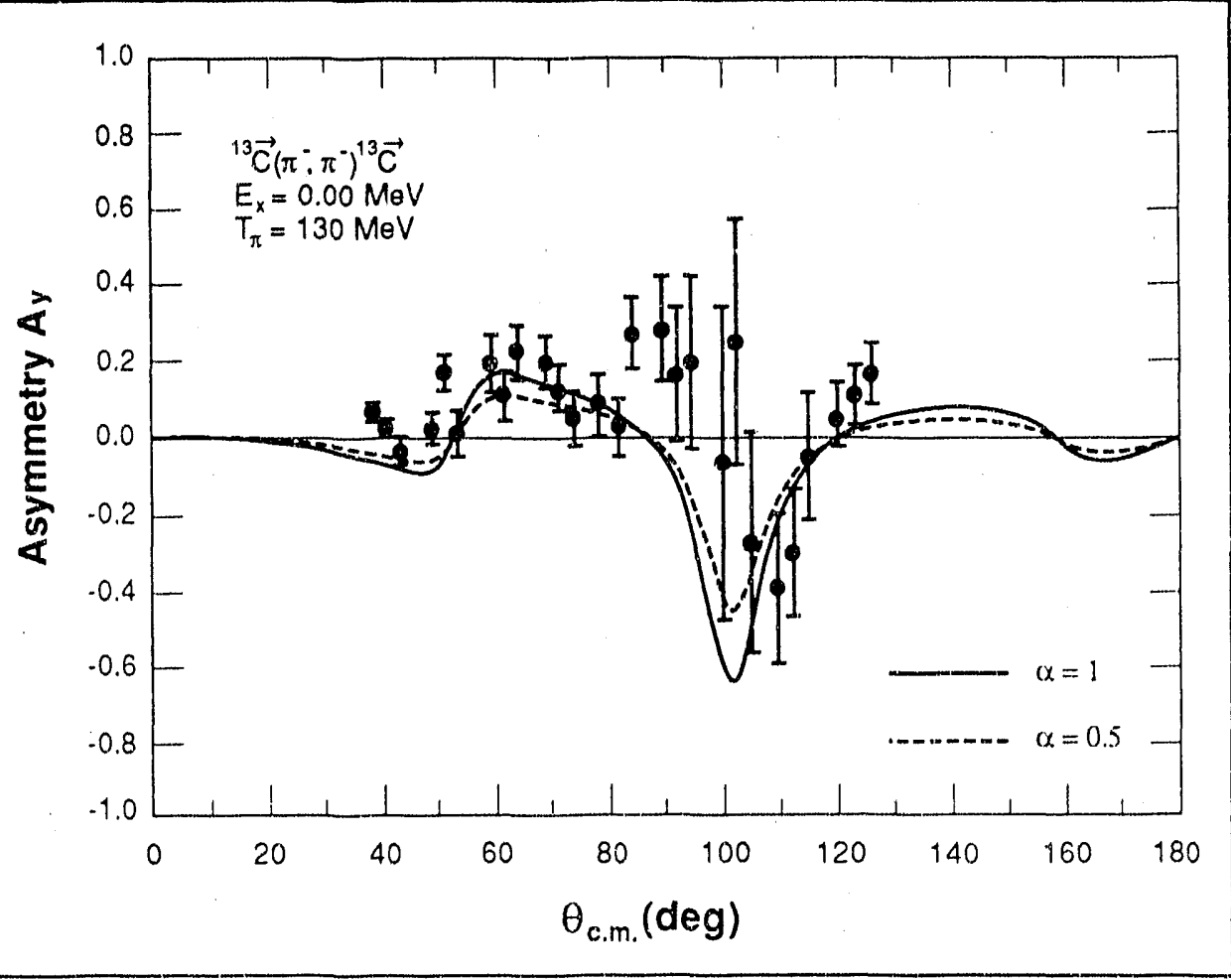

Fig. 1. Preliminary results from Exp. 1025: Asymmetries for elastic scattering of $\pi^{-}$from polarized ${ }^{13} \mathrm{C}$ at $T=130 \mathrm{MeV}$. Solid line: $D W I A$ calculation with full spin-transfer strength; dashed line: DWIA calculation with spindependent amplitude quenched by a factor of 0.5 .

Asymmetries consistent with zero were seen in an experiment of $\pi^{+}$scattering from ${ }^{15} \mathrm{~N}$ at $164 \mathrm{MeV}$ done this year at PSI. ${ }^{1}$ However, also at PSI, large asymmetries were observed for $\pi^{+}$scattering from ${ }^{6} \mathrm{Li}$ at 134 and $164 \mathrm{MeV}$. For ${ }^{15} \mathrm{~N}$, an asymmetry of zero is quite unexpected, because theoretical calculations with standard nuclear wave functions predict asymmetries that are quite large in at least some angular region and are rapidly varying with energy. ${ }^{2}$ Specifically, for ${ }^{15} \mathrm{~N}+\pi^{+}$at $164 \mathrm{MeV}$, the theoretical predictions do not resemble the data at all. Quite in contrast, the preliminary data from Exp. 1025 at $130 \mathrm{MeV}$ for ${ }^{13} \mathrm{C}+$ $\pi^{-}$are reproduced quite well by theoretical predictions. The solid line in Fig. 1 was obtained ${ }^{3}$ by DWIA calculations using the transition density amplitudes of 
Lee and Kurath. ${ }^{1}$ The dashed line was calculated with a quenching factor of 0.5 for the spin-dependent amplitude and the fit to the data is slightly worse. Quenching of the spin-dependent amplitudes is a well known phenomenon, ${ }^{5}$ but a fit to the near-zero asymmetries for ${ }^{15} \mathrm{~N}+\pi^{+}$would require an unrealistically strong quenching.

\section{References}

1. R. Tacik, E. T. Boschitz, R. Meier, S. Ritt, M. Wessler, K. Junker et al., Phys. Rev. Lett. 63, 1784 (1989).

2. Proceedings of the LAMPF Workshop on Physics with Polarized Nuclear Targets, Los Alamos National Laboratory report LA-10772-C (1986).

3. S. Chakravarti et al., Los Alamos, New Mexico, private communication (1989).

4. T. S. H. Lee and D. Kurath, Phys. Rev. C 21, 293 (1980).

5. I. S. Towncr, Phys. Rep. 155, 264 (1987). 
EXPERIMENT 1085 - LEP

\section{Pion Absorption on Deuterium Below $20 \mathrm{MeV}$}

\section{Arizona State Univ. Univ. of South Carolina, Univ. of Virginia}

Spokesmen: R. C. Alinehart (Univ. of Virginia) and 8. G. Ritchie (Anizona State Univ.)

Particivants: T. D. Averett, G. S. Blanpled, K. Glovanetti, R. C. Minehar, B. M Preedom, B. G. Ritchie, D. Rothenberger, L. C. Smith, and J. R. Tinsley
Since the long-range component of the nucleon-nucleon force is mediated by the exchange of virtual pions, an understanding of pion absorption in nuclei is a crucial underpinning to a thorough picture of how assemblies of nucleons form nuclei. The fundamental pion-absorption reaction is $\pi^{+}+d \rightarrow p+p$. A large amount of data exists for this process at energies from 20 to $300 \mathrm{MeV}$; these data have permitted a thorough understanding of the microscopic features of this reaction in that energy region. While many data exist for this process above $20 \mathrm{MeV}$, the only published data below $20 \mathrm{MeV}$ are those provided by a 23-yearold bubble-chamber experiment; that work provides the only total cross section data for the process at very low energies. No differential cross section data have been published, primarily because of the lack of a very low-energy pion beam and the difficulty of performing the time-reversed reaction near threshold.

Experiment 1085 was proposed to provide high-accuracy differential cross section measurements for $\pi^{+}+d \rightarrow p+p$ at energies below $20 \mathrm{MeV}$. The experiment was performed on the LEP channel, with a five-foot, crossed electric and magnetic field velocity filter to enhance the purity of the resulting pion beams. The detector system consisted of three E-dE telescopes of plastic scintillator at conjugate angles to three other E-dE telescopes as in Exp. 131. A deuterated scintillator served as target and beam flux and purity monitor.

Data were taken above $20 \mathrm{MeV}$ to check the combined operation of the channel, separator, target, and detection system. Below $20 \mathrm{MeV}$, the LEP channel was tuned to deliver a $20-\mathrm{MeV}$ pion beam to the separator, with various degraders inserted to lower the pion beam energy to the desired value. The beam was continuously sampled with the scintillator target during the data runs to provide an accurate determination of the pion fraction of the low-energy beam for each low-energy beam.

Beams of $20,15,10,6.5,5$, and $3.5 \mathrm{MeV}$ were developed, with gradually diminishing pion fractions for each of the low-energy beams below $20 \mathrm{MeV}$. By placing cuts on the pulse-height spectra of the E-dE telescopes and applying appropriate corrections for reaction losses as detailed in the publication for Exp. 131, relative cross sections have been determined from the replayed data. The $20-\mathrm{MeV}$ cross sections are in excellent agreement with the published results, providing confirmation of the validity of the normalization techniques. The deuterated scintillator beam purity spectra are currently under analysis to determine the pion fraction precisely to yield the absolute normalization. It is anticipated that the resulting cross sections will have an overall uncertainty of less than $8 \%$ in all cases. 
EXPERIMENT 1098 LEP/Clamshell

\section{Energy Dependence of Low-Energy Pion Double Charge Exchange}

\author{
Los Alamos, Tel Aviv Univ., \\ Arizona State Univ., \\ TRIUMF
}

\footnotetext{
Spokesmen: H. W. Baer, M. J. Lettch (Los Alamos), and $Z$. Weinfeld (Tel Aviv Univ.)

Participonts: H. W. Baer, A. Klein, M. J. Leitch, C. S. Mishra, Z. Weinfeld, E. Plasetzky, J. R. Comiort, J. Tinsley, and D. H. Wright
}

The objective of Exp. 1098 is to determine the energy and angular dependences of forward-angle differential cross sections of double-isobaric-analog state (DIAS) transitions and of ground-state (g.s.) transitions. We are performing measurements on selected $f_{7 / 2}$ shell nuclei ${ }^{42,44,48} \mathrm{Ca},{ }^{46,501} \mathrm{Ti}$, and ${ }^{54} \mathrm{Fe}$ in the incident energy interval 25 to $65 \mathrm{McV}$.

In the relatively short data-taking period allotted in 1989, we measured forward-angle cross sections at $50 \mathrm{MeV}$ on ${ }^{44} \mathrm{Ca}$. At this energy, there are systematic data available on ${ }^{14} \mathrm{C},{ }^{18} \mathrm{O},{ }^{26} \mathrm{Mg},{ }^{34} \mathrm{~S}$, and ${ }^{42} \mathrm{Ca} .{ }^{1-5}$ The first attempt to measure a DIAS transition at $50 \mathrm{MeV}$ in a $T>1$ nucleus was for ${ }^{48} \mathrm{Ca} .{ }^{6}$ The attempt failed because of poor signal/background and an unexpectedly small cross section. Since the DIAS in $T>1$ nuclei are excited states in the residual nuclei, such transitions are more difficult to measure because of the background of nonanalog transitions. In our 1989 run, we made the first successful measurement of a DIAS angular distribution at $50 \mathrm{MeV}$ for a $T>1$ nucleus. The DIAS of ${ }^{44} \mathrm{Ca}$ resides at $9.3 \mathrm{MeV}$ in ${ }^{44} \mathrm{Ti}$. Simultancously, we measured the transition to the ${ }^{14} \mathrm{Ti}$ ground state. This is also a $0^{+} \rightarrow 0^{+}$transition, but it requires an isospin change of two units.

The g.s. transitions are interesting in view of recent theoretical studies, ${ }^{7}$ which show that in a zero-seniority model of $\int_{7 / 2}$ shell nuclei, the DIAS and g.s. transitions are directly related. The DIAS transitions involve two amplitudes denoted by $A(\theta)$ and $B(\theta)$, whereas the g.s. transition involves only $B(\theta)$. In the zero-seniority model for the $f_{7 / 2}$ shell, the relative weightings of $A$ and $B$ in the transition amplitude for the DIAS are $A-\frac{1}{9} B$ for ${ }^{44} \mathrm{Ca}$ and $A+B$ for ${ }^{42} \mathrm{Ca}$. The g.s. transition of ${ }^{44} \mathrm{Ca}$ is predicted to have a differential cross section of $1.58 \mathrm{~B}^{2}$. The new data allow us to evaluate this model at $50 \mathrm{MeV}$, comparing for the first time DIAS and g.s. angular distributions.

Spectra measured for ${ }^{44} \mathrm{Ca}$ are shown in Fig. 1. One can see that at $25^{\circ}$ and $40^{\circ}$ the g.s. and DIAS transitions dominate the spectrum between 0- and $25-\mathrm{McV}$ excitation. At $70^{\circ}$, both states have greatly reduced cross sections, and a new peak at $6.5 \pm 0.2 \mathrm{MeV}$ is evident. Comparing the $25^{\circ}$ spectrum of Fig. 1 with that for ${ }^{48} \mathrm{Ca}$ shown in Ref. 6 , it is remarkable to note the difference in the DIAS signal/background. The much cleaner spectrum for ${ }^{44} \mathrm{Ca}$ made it possible to measure the angular distribution for the DIAS. Its excitation energy is most reliably extracted from the $25^{\circ}$ data, from which we obtain $9.3 \pm 0.2 \mathrm{MeV}$. This value agrees with the value of $9.31 \mathrm{MeV}$ for the lowest $T=2$ state in ${ }^{44} \mathrm{Ti}^{8}{ }^{8}$

The preliminary data on ${ }^{44} \mathrm{Ca}$ are compared with those of ${ }^{14} \mathrm{C}$ at $50 \mathrm{MeV}$ in Fig. 2. The angular distributions for ${ }^{14} \mathrm{C},{ }^{18} \mathrm{O}$, and ${ }^{26} \mathrm{Mg}$ at $50 \mathrm{MeV}$ are quite similar in both shape and magnitude. ${ }^{1-4}$ From Fig. 2, it is evident that the two ${ }^{44} \mathrm{Ca}$ angular distributions are more sharply forward peaked compared to ${ }^{14} \mathrm{C}$. The curves shown in the figure are fits based on the functional form $d \sigma / d \Omega=A e^{\lambda(\cos \theta-1)}$, which we have used previously for extrapolation to $0^{\circ}$. The extrapolated $0^{\circ}$ cross section for the DIAS is approximately $2 \mu \mathrm{b} / \mathrm{sr}$, which is about one-half the value of all measured $T=1$ nuclei with $A \leq 42$. A factor 


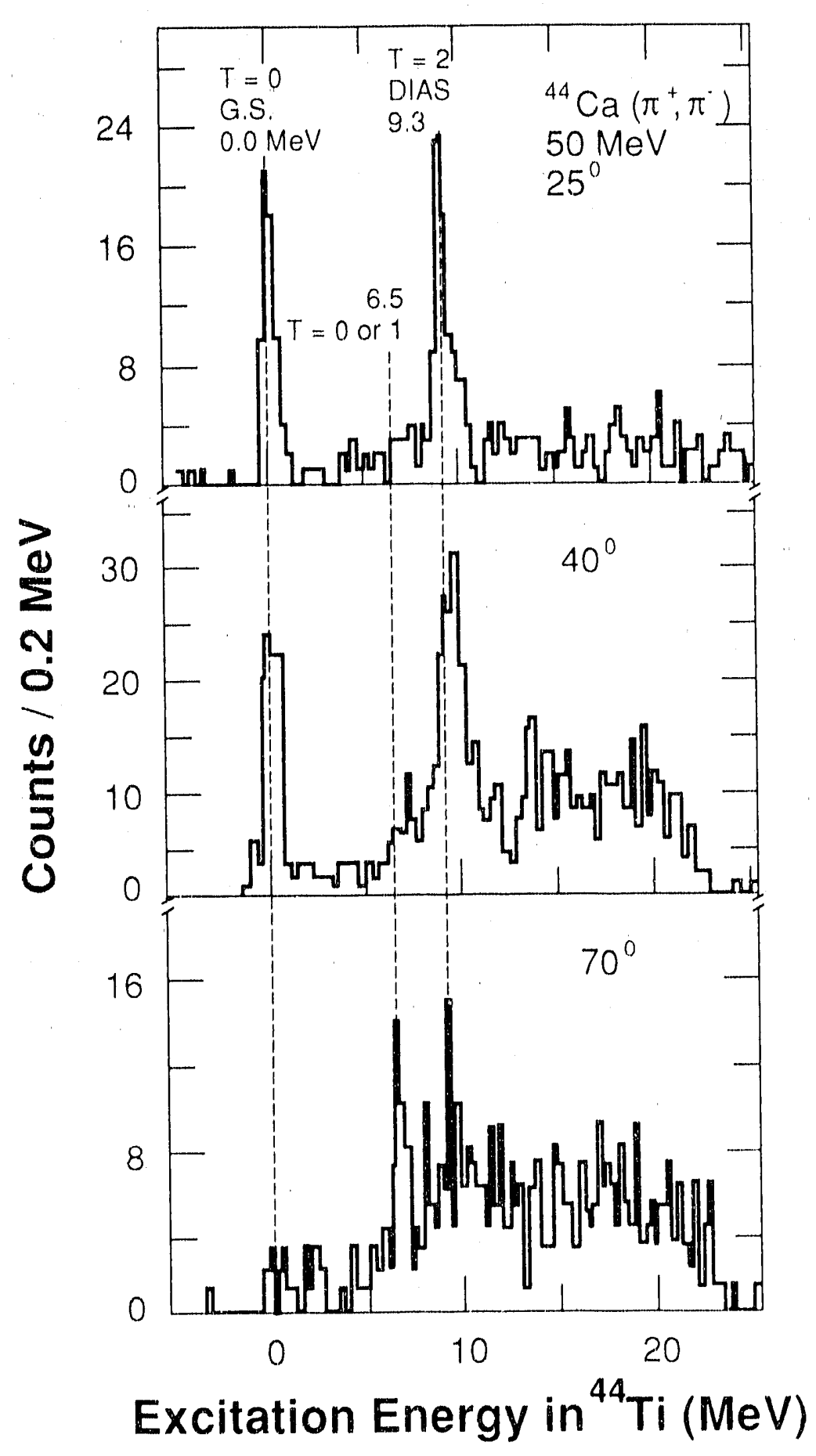

Fig. 1. The measured spectra of the ${ }^{44} \mathrm{Ca}\left(\pi^{+}, \pi^{-}\right)$reaction at $50 \mathrm{MeV}$. 


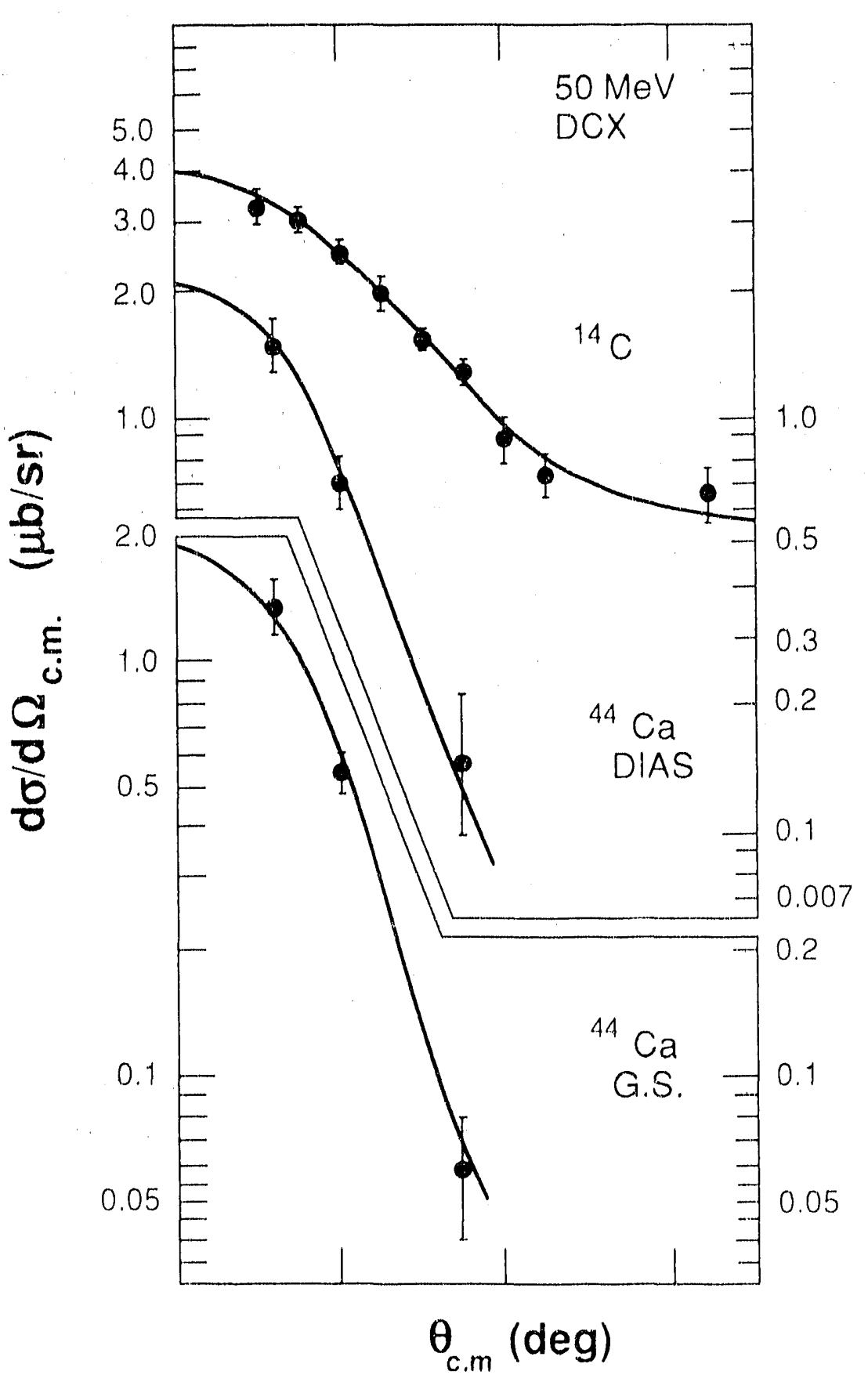

Fig. 2. Comparison of the preliminary cross section $50 \mathrm{MeV}$ on ${ }^{41} \mathrm{Ca}$ with those of ${ }^{14} \mathrm{C} .{ }^{2}$ The solid lines represent fits of the function $A e^{\lambda(\cos \theta-1)}$ to the data. 
of two reduction between ${ }^{44} \mathrm{Ca}$ and ${ }^{42} \mathrm{Ca}$ was also observed at $35 \mathrm{MeV} .{ }^{9}$ The g.s. cross section is also approximately $2 \mu \mathrm{b} / \mathrm{sr}$ at $0^{\circ}$, but by $70^{\circ}$, the g.s. cross section has dropped to a lower value than that of the DIAS. Thus, there is evidence that the angular dependence of the $B$-amplitude differs from that of the $A$-amplitude. These new data on ${ }^{44} \mathrm{Ca}$ will allow for a more detailed examination of the twoamplitude picture of low-energy double-charge-exchange (DCX) transitions.

\section{References}

1. I. Navon, M. J. Leitch, D. A. Bryman, T. Numao, P. Schlatter, G. Azuclos, R. Poutissou, R. A. Burnham, M. Hasinoff, J. M. Poutissou, J. A. MacDonald, J. E. Spuller, C. K. Hargrove, H. Mes, M. Blecher, K. Gotow, M. Moinester, and H. W. Bacr, Phys. Rev. Lett. 52, 105 (1984).

2. M. J. Leitch, E. Plasctzky, H. W. Bacr, J. D. Bowman, R. L. Burman, B. J. Dropesky, P. A. Gram, F. Irom, D. Roberts, G. A. Reloka Jr., J. N. Knudson, J. R. Comfort, V. A. Pinnick, D. H. Wright, and S. A. Wood, Phys. Rev. Lett. 54, 1482 (1985).

3. A. Altman, R. R. Johnson, U. Wienands, N. Hessey, B. M. Barnett, B. M. Forster, N. Grion, D. Mills, F. M. Rozon, G. R. Smith, R. P. Trelle, D. R. Gill, G. Sheffer, and T. Anderl, Phys. Rev. Lett. 55, 1273 (1985).

4. A. Altman et al., Bull. Am. Phys. Soc, 31, 802 (1986).

5. K. K. Seth, Nucl. Phys. A478, 591c (1988).

6. H. W. Bacr, M. J. Leitch, R. L. Burman, M. D. Cooper, A. Z. Zui, B. J. Dropesky, J. N. Knudson, J. R. Comfort, D. H. Wright, and R. Gilman, Phys. Rev. C 35, 1425 (1987).

7. N. Aucrbach, W. R. Gibbs, J. N. Ginocchio, and W. B. Kaufmann, Phys. Rev. C 38, 1277 (1988).

8. Table of Isotopes, 7th Edition, C. M. Lederer and V. S. Shirley, Eds. (Wiley, New York, 1978).

9. Z. Weinfeld, E. Piasetzky, H. W. Bacr, R. L. Burman, M. J. Leitch, C. L. Morris, D. H. Wright, S. H. Rokni, and J. R. Comfori, Phys, Rev, C 37, 902 (1988). 


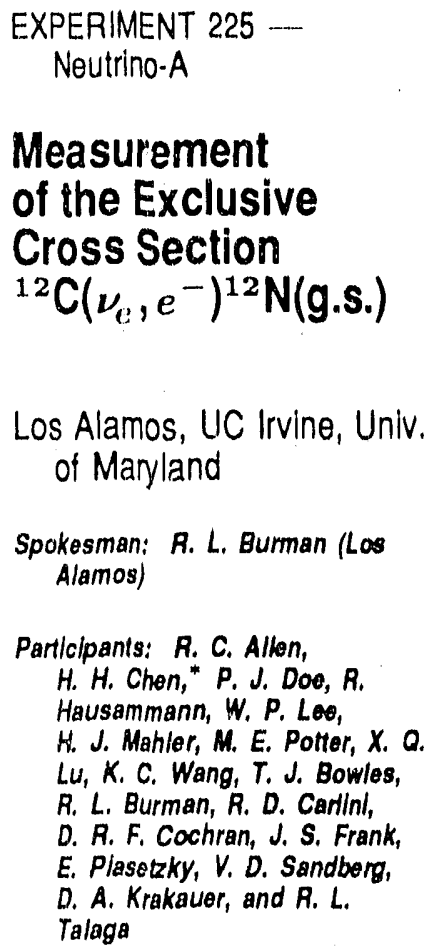

* deceasod
We report the measurement of the cross section for ${ }^{12} \mathrm{C}\left(\nu_{e}, e^{-}\right)^{12} \mathrm{~N}(\mathrm{~g} . \mathrm{s}$.$) , with$ $\nu_{e}$ from a beam stop neutrino source. This is the first observation of an electronneutrino-induced reaction between specific nuclear states. ${ }^{1}$ Such exclusive reactions are of interest in nuclear physics, astrophysics, and particle physics. Yet, to date, no experimental cross sections were available for neutrino-nucleus interactions to well-defined, two-body final states. This experiment observes such neutrino reactions at energies relevant for astrophysical observations and calculations. Our result provides useful checks of the theoretical calculations and serves as an experimental calibration.

This experiment was performed at the LAMPF Line-A beam stop as part of the Exp. 225 neutrino-electron-scattering experiment. At the beam stop, an isotropic flux of electron-neutrinos is produced by the decay of stopped $\mu^{+}$ (following stopped $\pi^{+}$decay). Data were collected between October 1983 and December 1986 with $1.12 \times 10^{23}$ protons incident on the beam stop, corresponding to a time-integrated neutrino flux of $(9.16 \pm 0.67) \times 10^{14} \nu_{e} / \mathrm{cm}^{2}$ at the geometric mean distance from the beam stop to the detector. ${ }^{2}$ The $\nu_{e}$ energy spectrum is described by the Michel distribution with a maximum energy of $52.8 \mathrm{MeV}$ and a peak energy at $35.2 \mathrm{MeV}$. The prompt electron from the ${ }^{12} \mathrm{C}\left(\nu_{e}, e^{-}\right)^{12} \mathrm{~N}$ reaction triggered the detector. The 17.4-MeV $Q$-value for the $\nu_{e}{ }^{12} \mathrm{C} \rightarrow e^{-12} \mathrm{~N}$ transition restricted the kinetic energy of the prompt electron to less than $35.4 \mathrm{MeV}$. The specific transition to the nitrogen ground state was then identified by the delayed coincidence of the ${ }^{12} \mathrm{~N}($ g.s. $)$ positron decay, ${ }^{12} \mathrm{~N} \rightarrow{ }^{12} \mathrm{C} e^{+} \nu_{e}$. Transitions to excited states of ${ }^{12} \mathrm{~N}$ were not identified as all of these states decay by prompt proton emission. Furthermore, neutrino reactions with all other possible nuclear targets produce nuclei with much longer lifetimes and smaller decay energies and so could not yield a decay-time spectrum comparable to the ${ }^{12} \mathrm{~N}(\mathrm{~g} . \mathrm{s}$.) signal. Therefore, the subsequent positron decay, with mean life $\tau=15.9 \mathrm{~ms}$ and endpoint energy $E_{\beta}=16.4 \mathrm{MeV}$, uniquely identifies the neutrino-induced transition to the nitrogen ground state.

The neutrino detector had a fiducial mass of 15 tons, primarily plastic, and was $85 \%{ }^{12} \mathrm{C}$ by mass. It was arranged in 40 identical close-packed $305 \times 305$ $\mathrm{cm}^{2}$ layers, each consisting of a plane of plastic scintillator followed by a flash chamber module (FCM). Each polystyrene scintillator plane $\left(2.64 \mathrm{gm} / \mathrm{cm}^{2}\right)$ was divided into four separate $76 \times 305-\mathrm{cm}^{2}$ counters. The pulse heights observed in individual counters were used to sample $d E / d x$ and were summed to determine the "visible energy," which was roughly $60 \%$ of the total energy deposited in the detector. Each FCM $\left(1.41 \mathrm{gm} / \mathrm{cm}^{2}\right)$ had ten panels of $520 \mathrm{He}$ - Ne-filled polypropylene flash tubes $\left(0.5 \times 0.6-\mathrm{cm}^{2}\right.$ cells $)$, alternating between horizontal and vertical orientation. The FCM's recorded the location and direction of charged-particle tracks. To detect the ${ }^{12} \mathrm{~N}($ g.s. $) \rightarrow{ }^{12} \mathrm{C} e^{+} \nu_{e}$ decay, the detector recorded the time and energy of all activities above $2.5-\mathrm{MeV}$ threshold in every scintillation counter during the $68.3 \mathrm{~ms}$ following each trigger; the time relative to the trigger was measured with $16.7 \mu$ s resolution. Details of the detector components and operational performance are given elsewhere. ${ }^{3}$ 
The detector was triggered by a coincidence of three consecutive scintillation layers with energy deposition between 1 and $16 \mathrm{MeV}$ per scintillator. Triggers were collected during the $0.75-\mathrm{ms}$ LAMPF beam spill (Beam-On) and for a longer period between spills (Beam-Off) in order to monitor cosmic-ray backgrounds. Calibration triggers on electrons from the decay of cosmic-ray muons stopped in the detector were interspersed with normal triggers throughout data collection. The data consist of 223,839 Beam-On and 562,739 Beam-Off triggers taken during $550.2 \mathrm{~h}$ of Beam-On and $2049.3 \mathrm{~h}$ of Beam-Otf live time. Obvious backgrounds, about $90 \%$ of all triggers, were eliminated by removing events not contained within the fiducial volume, without reconstructible tracks, or with activity in the anticoincidence or scintillators indicating stopped-muon decay during the $33 \mu \mathrm{s}$ prior to the trigger. Next, to enhance the electron sample relative to backgrounds, events were required to have $d E / d x<1.7 \times$ minimum-ionizing particles, to have hits in both views of the upstream FCM, and to contain only one or two groups of active contiguous scintillation counters. This reduced the data sample by an additional $65 \%$ to 7752 Beam-On events and 21,773 Beam-Off events.

Restrictions were imposed specifically to select prompt events satisfying ${ }^{12} \mathrm{C}$ inverse beta-decay kinematics. Events were required to have triggers with less than $24-\mathrm{MeV}$ visible energy, which corresponds to the $35-\mathrm{MeV}$ total energy kinematic limit for electrons from the reaction ${ }^{12} \mathrm{C}\left(\nu_{e}, e^{-}\right)^{12} \mathrm{~N}(\mathrm{~g}$.s.). The unique signature of ${ }^{12} \mathrm{~N}$ (g.s.) decay was used to identify the signal among the remaining 4059 Beam-On events and 10,772 Beam-Off events. The scintillators were examined for activity (energy deposition) during the time $50 \mu \mathrm{s} \leq t \leq 66.8 \mathrm{~ms}$ following the trigger. Because of the low energy of the ${ }^{12} \mathrm{~N}(\mathrm{~g} . \mathrm{s}$.) decay positron and the presence of intervening material (FCM's), the decay signal was expected to be visible in at most one scintillator. Because of a forward-backward ambiguity in the prompt track direction, the first and last scintillation counters of the prompt trigger were both searched. Nearly two-thirds of all prompt events had an activity that satisfied this criterion. The energy spectrum of delayed-energy deposition was used to help distinguish true positron decays from the much softer energy spectrum of random background activities. Activities with energies between 4.5 $\mathrm{MeV}$ and $12.0 \mathrm{MeV}$ were ${ }^{12} \mathrm{~N}$ (g.s.) decay candidates. This resulted in 304 ${ }^{12} \mathrm{C}\left(\nu_{e}, e^{-}\right)^{12} \mathrm{~N}(\mathrm{~g} . \mathrm{s}$.$) candidates in the Beam-On data set and 424$ candidates in the Beam-Off data. The number of candidates as a function of time following the prompt trigger is displayed in Fig. 1. The time spectrum is assumed to contain a contribution from the signal, the exponential decay of ${ }^{12} \mathrm{~N}($ g.s. $)$ with lifetime $\tau=15.87 \mathrm{~ms}$, and a contribution from a uniformly distributed background. The normalization of the signal and background contributions was determined by a maximum likelihood fit to the decay-time spectra. The time spectrum predicted by this estimate is plotted as a solid line on top of the data in Fig. 1(a). From the 304 Beam-On candidates, and the maximum likelihood fit, we identify a signal of $181 \pm 17^{12} \mathrm{C}\left(\nu_{c}, e^{-}\right)^{12} \mathrm{~N}(\mathrm{~g} . \mathrm{s}$.$) events.$ 


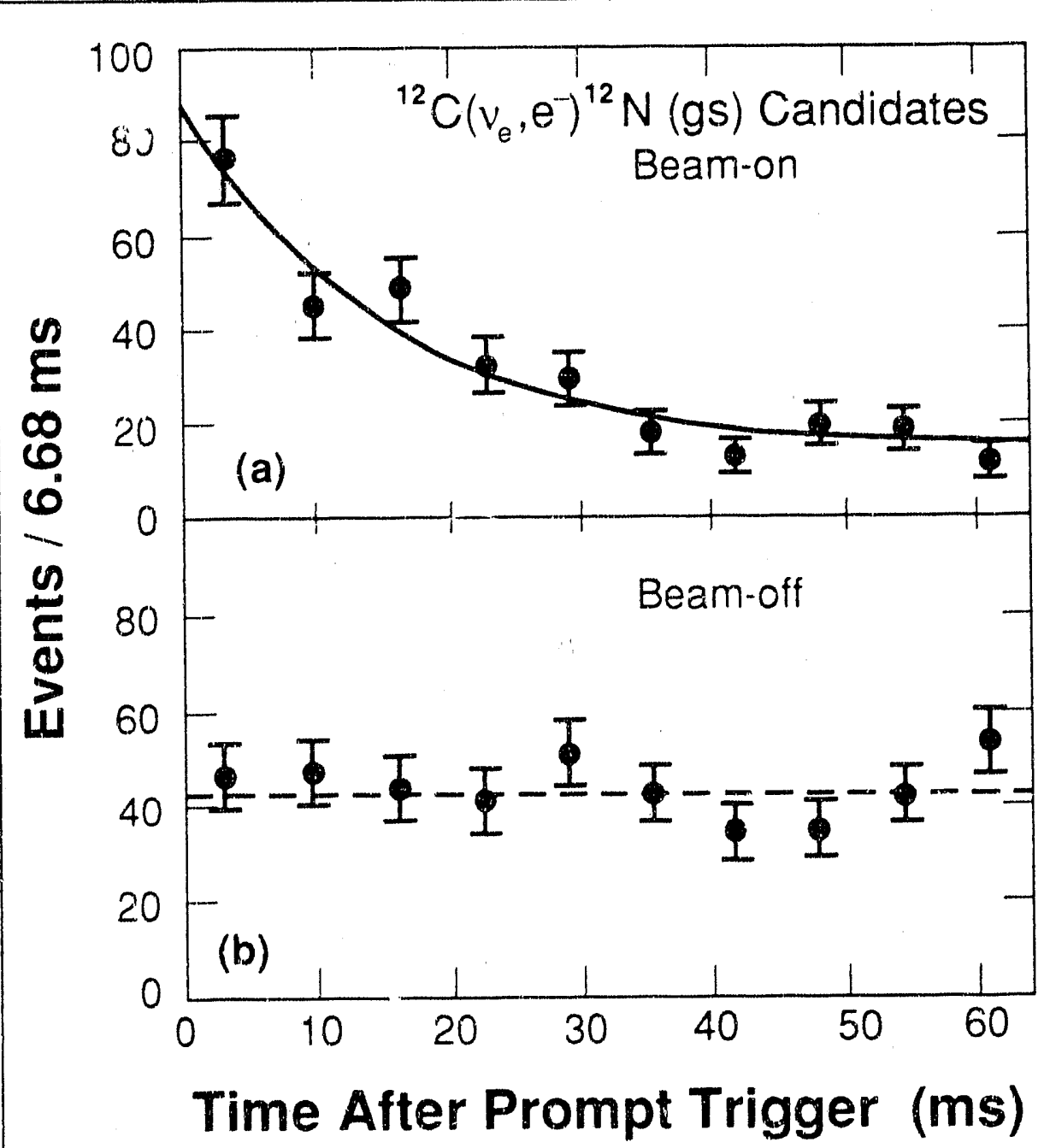

Fig. 1. Time spectrum of ${ }^{12} \mathrm{~N}$ decay candidates in bins of $6.68 \mathrm{~ms}$ following the prompt trigger. (a) Beam-On prompt events. The solid line is . he result of the maximum likelihood fit described in the text. (b) Beam-Off pr. npt events. The dashed line is the average of the ten biris.

Severa! tests were performed to study the delived coincidence time spectrum to investigate possible backgrounds or exprri

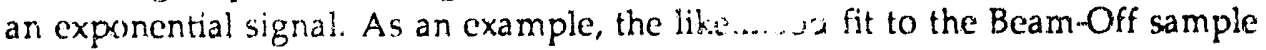
of 424 cano. (ates satisfying all of the above restrictions [Fig. 1(b)] found $17 \pm$ $2212 \mathrm{~N}$ (g.s.) docays, consistent with no signal. The rexults of the tests wome all consistent with a time-independent spectrum, thus ruling out backgroundi, hardware bias, und analysis bias as sources of the exponential decay. 
We performed a detailed Monte Carlo simulation of the detector response to the ${ }^{12} \mathrm{C}\left(\nu_{e}, e^{-}\right)^{12} \mathrm{~N}(\mathrm{~g} . \mathrm{s}$.) events to detcrmine the experimental detection effi.ciency and expected kinematic distributions. The prompt electron and delayed positron were simulated using the electron-photon transport code EGSA. All detector parameters surh as FCM performance, scintillator response, and random noise activities were taken from direct measurements and calibration data. Simulated events were translated into data format and passed through the off-line analysis. Thus, Monte Carlo was able to reproduce the measured characteristics of calibration triggers from stopped-muon decays, which verifies the correctness of the simulation algorithms.

The trigger electrons were generated according to the $d \sigma / d \Omega d E_{e}$ distribution calculated by Donnelly. ${ }^{1}$ The detection efficiency for these prompt electrons, including all restrictions and corrections, is c prompl $=9.2 \pm 0.6 \%$. The positron decay was included in the simulation, and for the timing and energy thresholds used in this analysis, the $e^{+}$were detected with cdetay $=32 \pm 2 \%$ efficiency. The overall detection efficiency was $c=\epsilon_{\text {promp }} * c_{\text {delay }}=3.0 \pm 0.2 \%$. The uncertainty in c properly reflects the combined systematic uncertainties of the entire Monte Carlo simulation. The deteclor contained $6.36 \pm 0.13 \times 10^{29} 1^{12} \mathrm{C}$ target nuclei. Combining the number of target nuclei, detection efficiencies, and $\nu_{e}$ flux iniensity described above with our detection of $181 \pm 17$ events, we determine the flux-weighted cross section

$$
\sigma\left[\nu_{e}{ }^{12} \mathrm{C}-v^{-12} \mathrm{~N}(\text { g.s. })\right]=[1.05 \pm 0.10(\text { stat }) \pm 0.10(\text { sys })] \times 10^{-41} \mathrm{~cm}^{2} .
$$

The statistical error included the systematic uncertainties of the fitting procedure and backgrounds; the systematic error included the uncertainties from the Monte Carlo determination of detection efficiencies (6\%), from the neutrino flux (7.3\%), and from the number of targets $(2 \%)$ added in quadrature. If we assume it the total cross section is proportional to $\left(E_{\nu}-Q\right)^{2}$, with $Q=17.4 \mathrm{Mc}^{\prime}$. .he flux-weighted cross section is equivalent to the total cross section for electronneutrinos with incident energy $E_{\nu}=35.0 \mathrm{MeV}$.

The total cross section for ${ }^{12} \mathrm{C}\left(\nu_{e}, e^{-}\right)^{12} \mathrm{~N}(\mathrm{~g}$.s. $)$ has been calculated by several authors who are all in general agreement. Donnelly." calculates the flux-averaged cross section to be $\sigma=0.94 \times 10^{-11} \mathrm{~cm}^{2}$ for neutrinos from stopped-muon decay. Fukugita, Kohjama, and Kubodera ${ }^{5}$ calculated this quantity to be $\sigma=0.92 \times$ $10^{-41} \mathrm{~cm}^{2}$. Mintz and Pourkaviani found the flux-averaged cross section $\sigma=$ $0.90 \times 10^{-41} \mathrm{~cm}^{2}$. We found good agreement between our experimental result and these theoretical estime ies.

\section{References}

1. Mrasurement of the rolated reaction, ${ }^{12} \mathrm{C}\left(\nu_{i^{\prime}}, \mu^{-}\right)^{12} \mathrm{~N}(\mathrm{~g} . \mathrm{s}$.$) , is underway by a$ different group, R. W. Manweiler et al., in Proceedings of the Third Conference on the Intersections Between Particle and Nuclear Physics, Rockport, Maine, 1988, G. Bunce, Ed. [AIP Cor.f. Proc. 176, 912 (1989)]. 
RESEARCH

Nuclear and Particle Physics

2. R. C. Allen, H. H. Chen, M. E. Potter, R. L. Burman, J. B. Donahue, D. A. Krakauer et al., Nucl. Instrum. and Methods A 284, 347 (1989); R. L. Burman, M. E. Potter, and E. S. Smith, Nucl. Instrum. and Methods A 291, 621 (1990).

3. R. C. Allen, V. Bharadwaj, N. Briscoe, G. A. Brooks, H. H. Chen, R. S. DeLay et al., Nucl. Instrum. and Methods A 269, 177 (1988).

4. T. W. Donnelly, Phys. Lett. 43B, 93 (1973), and private communication (1987).

5. M. Fukugita, Y. Kohyama, and K. Kubodera, Phys. Lett. 212B, 139 (1988).

6. S. L. Mintz and M. Pourkaviani, Phys. Rev. C40, 2458 (1989). 
EXPERIMENT 225 Neutrino-A

\section{A Study of Neutrino- Electron Elastic Scattering}

Los Alamos, UC Irvine, Univ. of Maryland

Spokesman: R. L. Burman (Los
Alamos)
Participants: R. C. Allen,
H. H. Chen, ${ }^{*}$ P. J. Doe, R.
Hausammann, W. P. Lee,
H. J. Mahler, M. E. Potter, X. O.
Lu, K. C. Wang, T. J. Bowles,
R. L. Burman, R. D. Cantinl,
D. R. F. Cochran, J. S. Frank,
E. Piasetzky, V. D. Sandberg,
D. A. Krakauer, and R. L.
Talaga

"jeceased
The Exp. 225 collaboration (University of California at Irvine, Los Alamos, and University of Maryland) has completed the analysis of the electron-neutrinoelectron elastic-scattering experiment. The elastic-scattering reaction, $\nu_{e} e \rightarrow \nu_{f}=$, first observed in $1985,{ }^{1}$ is mediated via both the charged-current and neutralcurrent interactions, in contrast to the neutral-current reactions, $\nu_{\mu} e-\nu_{\mu} e$ and $\bar{\nu}_{\mu} e \rightarrow \bar{\nu}_{\mu} e$. If the outgoing neutrino from the charged-current interaction is the same as the outgoing neutrino from the neutral-current interaction, then these two interactions will interfere. The cross section for the reaction, $\nu_{e} e \rightarrow \nu_{e} e$, can be written as the sum of a neutral-current term, a charged-current term, and an interference term between charged and neutral currents. ${ }^{2}$ Assuming $\mu-e$ universality, the neutral-current term is equal to $\sigma\left(\nu_{\mu} e\right)$, which has been measured by several experiments, ${ }^{3,4}$ and the charged-current term is defined to be $2 G_{F}^{2} m_{e} E_{\nu} / \pi$, where the Fermi constant, $G_{F}$, is measured by muon-decay experiments, and $E_{\nu}$ is the laboratory energy of the incident neutrino. The interference term can be expressed as $I \times G_{F}^{2} m_{e} E_{\nu} / \pi$, where $I$ measures the interference strength. Thus, the sign and magnitude of the interference between chargedand neutral-current amplitudes can be deduced from an accurate measurement of the $\nu_{e} e$ cross section. In the Weinberg Salam - Glashow (WSG) model of electroweak interactions, the neutral-current interaction is mediated by the $Z$ boson, and the charged-current interaction by the $W^{ \pm}$bosons. A destructive value of the interference is predicted ( $\left.I_{\mathrm{WSG}}=-2+4 \sin ^{2} \theta_{W}\right)$, which lowers the $\nu_{e} e$ cross section by $40 \%$ compared with that in the absence of interference.

This experiment used the intense flux of $\nu_{e}$ available at the LAMPF LineA proton beam stop. These neutrinos are produced from stopped $\pi^{+}$decays, which make 30-MeV $\nu_{\mu}$, followed by stopped $\mu^{+}$decays, which make $\bar{\nu}_{\mu}$ and $\nu_{e}$ with end-point energies of $53 \mathrm{MeV}$; the mean $\nu_{e}$ energy is $31.7 \mathrm{MeV}$. For our 20$\mathrm{MeV}$ electron detection threshold, the recoil electron from $\nu_{x}$ e scattering (where $\nu_{x}$ refers to any of the above three types of neutrino) is confined urithin a $10^{\circ}$ cone with respect to the incident neutrino direction. Thus, the elastic scattering signal is forward-peaked, whereas backgrounds from both cosmic rays and the accelerator are essentially isotropic.

Details of the detector have been previously published. ${ }^{1,5}$ Briefly, a system of active and passive shielding surrounded a 15 -metric-ton central tracking detector. The active shicld, which consisted of 594 multiwire proportional counters arranged in four layers that cover all surfaces of the detector, was used to reject incoming charged particles. Within the active shield, a $100-\mathrm{g} / \mathrm{cm}^{2}$ layer of steel and lead was used to convert and attenuate residual gammas from cosmic-ray muons. The fine-grained central detector was used to measure the energy and track direction of the recoil electrons; it was arranged in a sandwich structure of forty identical layers. Each $305-\mathrm{cm}$ high by $305-\mathrm{cm}$ wide layer consisted of four pieces of $76-\mathrm{cm}$ wide by $2.5-\mathrm{cm}$ thick plastic scintillator, each viewed by a single $12.7-\mathrm{cm}$ diameter photomultiplier tube. Each scintillation layer was followed by ten panticls of flush chambers, cach containing 520 polypropylene flash tubes filled with a mixture of helium-neon gas, arranged in alternating $X$ and $Y$ 
planes. The total number of target electrons in the central detector was $4.94 \pm$ $0.05 \times 10^{30}$.

The trigger rate of less than $0.1 \mathrm{~Hz}$ was dominated by cosmic-ray and accelerator backgrounds. To reduce these backgrounds, restrictions were imposed on the data. First, cosmic-ray events were eliminated by removing events with substantial activity in the active shield, or in the scintillation counters during the $32-\mu \mathrm{s}$ preceding the trigger. Next, a fiducial region excluding the outer $5 \mathrm{~cm}$ of the detector was defined in order to remove gammas that converted in the inner shield. This restriction also removed incoming charged particles not identified by the active shield and insured that the remaining events were completely contained within the central detector.

Nonelectron backgrounds were reduced by removing events with a $d E / d x$ greater than $4 \mathrm{MeV} / \mathrm{cm}$ (twice minimum ionizing) in any layer and events with less than $1.2 \mathrm{McV} / \mathrm{cm}$ in any middle layer. Events with more than two clusters of adjacent scintillators, or with a poor quality fit to a track, were assumed to be associated with low-energy neutrons and were eliminated. Finally, the data taken during the beam-off gate were used to determine and then to subtract the cosmic-ray background, lcaving a total of $1492 \pm 76$ beam-associated electron events. The observed energy and angular distributions of these recoil electrons are shown in Figs. 1 and 2, respectively.

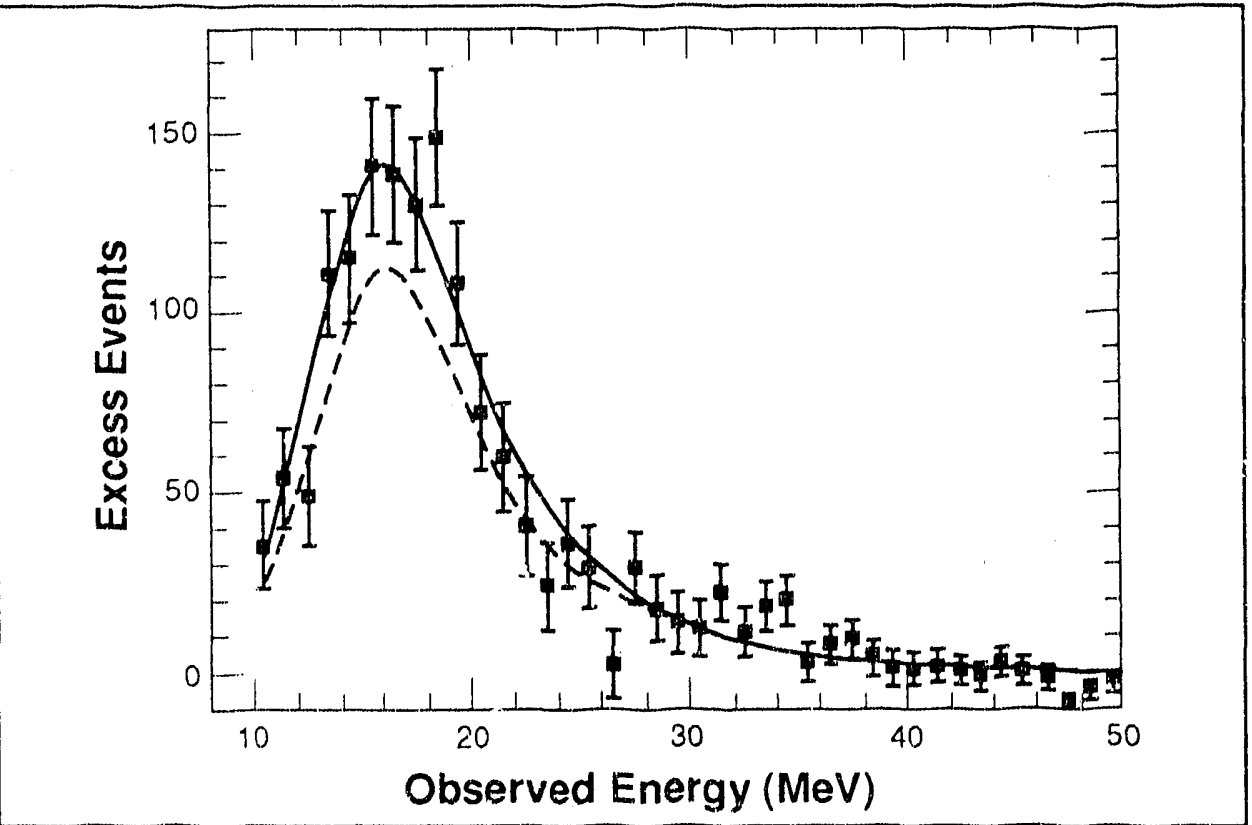

Fig. 1. The observed energy spectrum of the beam-associated recoil electrons. Due to the presence of the fiash chambers, only about $2 / 3$ of the total particle energy is deposited in the scintillators. The solid line indicates the fit to the ivionie Cario distributions for the expected signai ana 'oackgrounds. The aiviea line indicates the background only. 


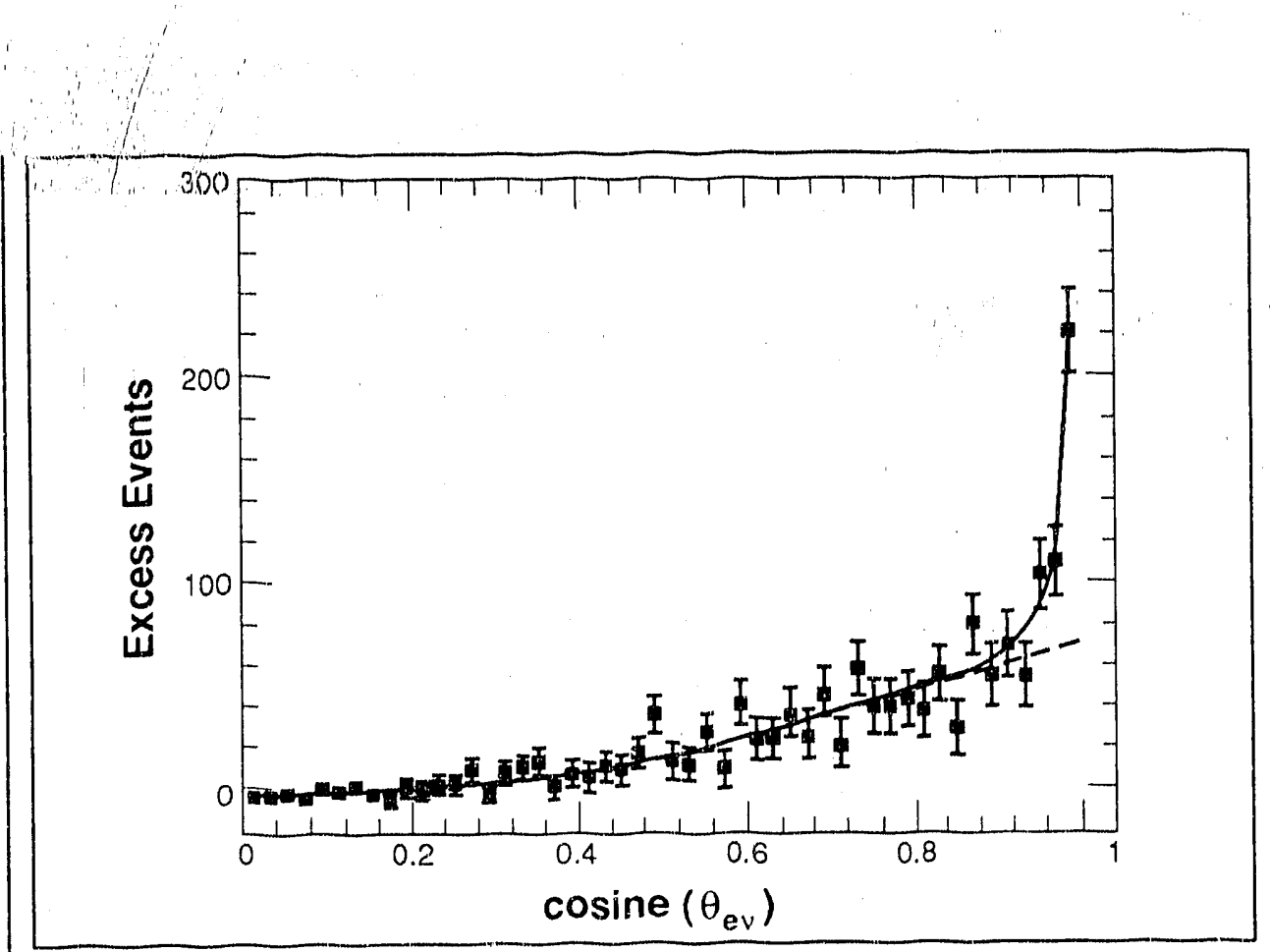

Fig. 2. The angular spectrum of the beam-associated recoil electrons. Here, $0_{e v}$ is the angle between the incident neutrin and the reconstructed recoil electron. The solid line indicates the fit to the Monte Carlo distributions for the expected signal and backgruunds. The dotted line indicates the background only.

A multiparameter fit to the two-dimensional distribution of recoil angle vs observed energy was used to determine the elastic scattering signal. The components of the fit were given by Monte Carlo expectations for the background and signal shapes. The overall normalization of each distribution was allowed to vary with the total number of events fixed. The distributions used in the fit corresponded to $\nu_{x}$ e clastic scattering, $\nu_{e}{ }^{12} \mathrm{C}$ and $\nu_{t}{ }^{13} \mathrm{C}$ inverse beta decay, and neutron-induced background. The $\nu_{e}{ }^{13} \mathrm{C}$ distributions represented the combination of all neutrino-nuclei interactions in the detector with low threshold values $\left({ }^{13} \mathrm{C},{ }^{27} \mathrm{Al},{ }^{35} \mathrm{Cl}\right)$. The fit to the observed energy and angular distributions is shown as solid lines in Fig. 1 and 2. This fit results in a measurement of $295 \pm 35$ $\nu_{x} e$ events, $626 \pm 71 \nu_{e}{ }^{12} \mathrm{C}$ events, and $571 \pm 136$ remaining background events, of which approximately 140 are expected from other neutrino-nuclei interactions and the remainder attributed to neutron-induced backgrounds. In addition, the $\nu_{e}{ }^{12} \mathrm{C} \rightarrow e^{-{ }^{12}} \mathrm{~N}$ (g.s.) reaction, which is the main source of beam-associated background, was simultaneously measured by detection of the delayed positron from the ${ }^{12} \mathrm{~N} \rightarrow{ }^{12} \mathrm{C} c+\nu_{e}$ beta decay; this measurement gave results consistent with the distribution fit.

The Monte Carlo used to simulate the energy and angular distributions for the above fit was also used to compute the detector efficiency for recoil electrons. This simulation, based upon the electron-gamma-shower code (EGS4), took into 
account light attenuation in the scintillators and photoelectron statistics. Uncertainties in the detector-energy scale, flash-chamber performance, and randomnoise rate leads to a combined systematic uncertainty of $\pm 5 \%$ in the detertion efficiency. The overall detection efficiency for $\nu_{e} e$ scattering was found to be $16.5 \pm 0.8 \%$. The largest systematic error came from uncertainty in the neutrino flux from the beam stop. An experiment to measure the number of stopped $\pi^{+}$ decays followed by stopped $\mu^{+}$decays per incident proton in an instrumented beam stop of various materials was performed at LAMPF. ${ }^{6}$ These results were used to calibrate a Monte Carlo program ${ }^{7}$ that provided a detailed simulation of the actual proton beam stop at LAMPF. For our entire beam exposte, the total resulting time-integrated neutrino flux was computed to be $9.16 \pm 0.67 \times 10^{14}$ $\nu_{e} \mathrm{~cm}^{2}$, at the average detector distance from the beam stop of $898 \mathrm{~cm}$. When the uncertainty in the flux and the detection efficiency are added in quadrature, the total systematic uncertainty is $8.8 \%$.

The $295 \pm 35$ neutrino-electron events include contributions from $\nu_{\mu} e$ and $\bar{\nu}_{\mu}$ e. Using measured cross sections from previous experiments, ${ }^{3,4} 27.4 \pm 4.7 \nu_{\mu} e$ and $33.5 \pm 5.9 \bar{\nu}_{\mu} e$ events were subtracted, leaving $234 \pm 35 \nu_{e} e$ events; in the absence of interference, $448 \pm 40 \nu_{e} e$ events would be expected. From the 234 $\nu_{e} e$ events, the total cross section is

$$
\sigma\left(\nu_{e} e\right)=9.9 \pm 1.5(\mathrm{stat}) \pm 1.0(\text { syst }) \times 10^{-42} \mathrm{~cm}^{2} \times E_{\nu}(\mathrm{GeV})
$$

or $\sigma\left(\nu_{e} e\right)=1.15 \pm 0.21 \times G_{F}^{2}, m_{e} E_{\nu} / \pi$. The charged-current contribution to this cross section is $2 G_{F}^{2}, m_{e} E_{\nu} / \pi$. From the muon-neutrino electron elastic-scattering experiments, ${ }^{3,4}$ the neutral-current contribution is measured to be $0.22 \pm 0.03$ $\times G_{F}^{2}, m_{e} E_{\nu} / \pi$. By subtracting the charged-and neutral-current contributions from the measured $\nu_{c} e$ cross section, the interference strength is determined to be

$$
I=-1.07 \pm 0.17 \text { (stat) } \pm 0.12 \text { (syst) } .
$$

For the world-averaged value of $\sin ^{2} \theta_{W}=0.230$, the Standard Model predicts a value of $I_{\mathrm{WSG}}=-1.08$.

In conclusion, we have measured the $\nu_{e} e \rightarrow \nu_{e} e$ cross section to sufficient precision (18\%) to determine the interference between $W$ and $Z$ exchange. This first measurement of the sign and magnitude of the coherent interference is in excellent agreement with the predictions of the WSC electroweak model.

The experimenters wish to acknowledge the efforts of N. Briscoe, J. Sena, T. N. Thompson, and S. DeLay in the construction and operation of Exp. 225.

\section{References}

1. R. C. Allen, V. Bharadwaj, G. A. Brooks, H. H. Chen, P. J. Doe, R. Hausammann et al., Phys. Rev. Lett. 55, 2401 (1985).

2. B. Kayser, E. Fischbaih, S. P. Rosen, and H. Spivack, Phys. Rev. D 20, 87 (1979). 
3. J. Dorenbosch, F. Udo, J. V. Allaby, U. Amaldi, G. Barbiellini, M. Baubillier et al., Z. Phys. C 41, 567 (1989).

4. K. Abe, L. A. Ahrens, K. Amako, S. H. Aronson, E. W. Beier, J. L. Callas et al., Phys. Rev. Lett. 62, 1709 (1989).

5. R. C. Allen, V. Bharadwaj, N. Briscoe, G. A. Brooks, H. H. Chen, R. S. DeLay et al., Nucl. Instrum. and Methods A 269, 177 (1988).

6. R. C. Allen, H. H. Chen, M. E. Potter, R. L. Burman, J. B. Donahue, D. A. Krakauer et al., Nucl. Instrum. and Methods A 284, 347 (1989).

7. R. L. Burman, M. E. Potter, and E. S. Smith, Nucl. Instrum. and Methods A291, 621 (1990). 
EXPERIMENT 645 Neutrino-A

\section{A Search for Neutrino Oscillations}

\section{Ohio State Univ., Argonne, Caltech, Lawrence Berkeley Laboratory, Louisiana State Univ., Los Alamos}

Spokesmen: R. L. Imlay and W. J. Metcalf (Louisiana State Univ.)

Partlcipants: L. S. Durkin, R. W. Harper, T. Y. Ling, J. W. Mitchell, T. A. Romanowski, E. S. Smith, M. Timko, S. J. Freedman, J. Napolitano, B. K. Fujikawa, R. McKeown, K. T. Lesko, W. C. Chol, A. Fazely, R. L. Imlay, W. J. Metcalt, R. D. Carlini, J. B. Donahue, G. T. Garvey, and V. Sandberg
Experiment 645 began its physics run in June of 1987. The experiment searches for the appearance of electron antincutrinos, which can result from the oscillation of muon antineutrinos produced in the LAMPF A6 beam stop.

The results of the first run, which took place between June and December 1987, have been published in the October 1988 issue of Physical Review Letters. A total of 5100 Coulombs of protons was incident on the LAMPF beam stop, yielding $4.2 \times 10^{*}$ neutrinos incident on the 20-ton detector. After subtracting the cosmic-ray rate of $0.10 \pm 0.02$ events per day, there was a beam excess of $12.3 \pm$ 4.7 events. These events are well explained by background neutrino interactions, and there is no evidence for neutrino oscillations. The $90 \%$ confidence level limits are $\delta m^{2}<0.11 \mathrm{eV}^{2}$ for maximal mixing and $\sin ^{2}(2 \theta)<0.014$ for large $\delta m^{2}$. An analysis, which exploits neutron detection, yields comparable limits.

The experiment ran again in 1988 and 1989, accumulating another 8400 Coulombs of protons on target for a total exposure of 13,500 Coulom?'s. The preliminary results are consistent with the analysis of the 1987 data.

Analysis of the entire data set should result in approximately a factor of two better limits on $\delta m^{2}$ and $\sin ^{2}(20)$, assuming that there are no surprises in the data. We will also search for oscillation of decay-in-flight neutrinos in our data and set limits on various exotic processes such as $\pi^{0} \rightarrow \nu \bar{t}$. 
EXPERIMENT 1173 Neutrino-A

\section{Search for Neutrino Oscillations with High Sensitivity in the Appearance Channels $\nu_{\mu} \rightarrow \nu_{e}$ and $\bar{\nu}_{\mu} \rightarrow \bar{\nu}_{e}$}

UC Irvine, UC Riverside, CEBAF, Los Alamos, Louisiana State Univ., Univ. of New Mexico, Univ. of Pennsylvania, Temple Univ.

Spokesman: W. C. Louis (Los
Alamos)
Particlpants: X. O. Lu, G. Yodh,
S. Y. Fung, J. H. Kang, B. C.
Shen, G. J. VanDalen, R. C.
Carlini, J. J. Napolitano, J.
Amann, H. Baer, R. Burman,
J. Donahue, W. Foreman,
G. T. Garvey, M. Hoehn,
T. Kozlowskl, D. M. Lee,
W. C. Louls, J. McClelland,
E. C. MIIner, M. Oothoudt,
V. Sandberg, M. Schillacl, N.
Tanaka, R. Werbeck, D. H.
White, D. Whitehouse, A. Fazely,
M. Brooks, B. B. Dieterte, C. P.
Leavitt, R. Reeder, A. K. Mann,
and W. K. McFarlane

The Liquid Scintillator Neutrino Detector (LSND) experiment will search concurrently for $\nu_{\mu} \rightarrow \nu_{e}$ and $\bar{\nu}_{\mu} \rightarrow \bar{\nu}_{e}$ oscillations, with high sensitivity, at LAMPF. Neutrino oscillations, if obscrved, would have a major impact on particle, nuclear, and astrophysics, as they would imply that lepton number is not conserved and that neutrinos have mass and contribute substantially to the mass of the universe. Other physics to be obtained include measurements of the chargedcurrent reactions $\nu_{e} C \rightarrow e^{-N}$ and $\nu_{\mu} C \rightarrow \mu^{-} N$, of the inolastic neutral current reaction $\nu C \rightarrow \nu C^{*}(15.11 \mathrm{McV} \gamma)$, and a search for the rare decays $\pi^{\prime \prime} \rightarrow \nu \nu^{\prime}$ and $\eta \rightarrow \nu \nu^{\prime}$.

The detector, shown in Fig. 1, consists of a tank with 200 tons of dilute liquid scintillator with 85010 -in photomultiplier tubes mounted on the inside tank, covering $28 \%$ of the surface. If oscillations occur, then $\nu_{e}$ and $\bar{\nu}_{e}$ neutrinos are produced, which interact in the detector via the reactions $\nu_{e} C \rightarrow e^{-} N$ and $\bar{\nu}_{e} p \rightarrow$ $e^{+} n$, respectively. Both Čerenkov and scintillation lipht will be detected and will enable us to determine fairly precisely the angle, position, energy, and time of the produced electron. The tank will reside inside the existing Exp. 645 veto shield and the experiment will make use of the present A6 beam stop neutrino source. After two years of data collection, $90 \%$ confidence level limits on $\nu_{\mu}\left(\bar{\nu}_{\mu}\right) \rightarrow \nu_{e}\left(\bar{\nu}_{e}\right)$ mixing strengths of $2.7(2.7) \times 10^{-4}$ can be obtained for all $\Delta m^{2}>1 \mathrm{cV}^{2}$. This experiment will, therefore, provide the world's best terrestrial limits on $\nu_{\mu} \rightarrow \nu_{e}$ oscillations.

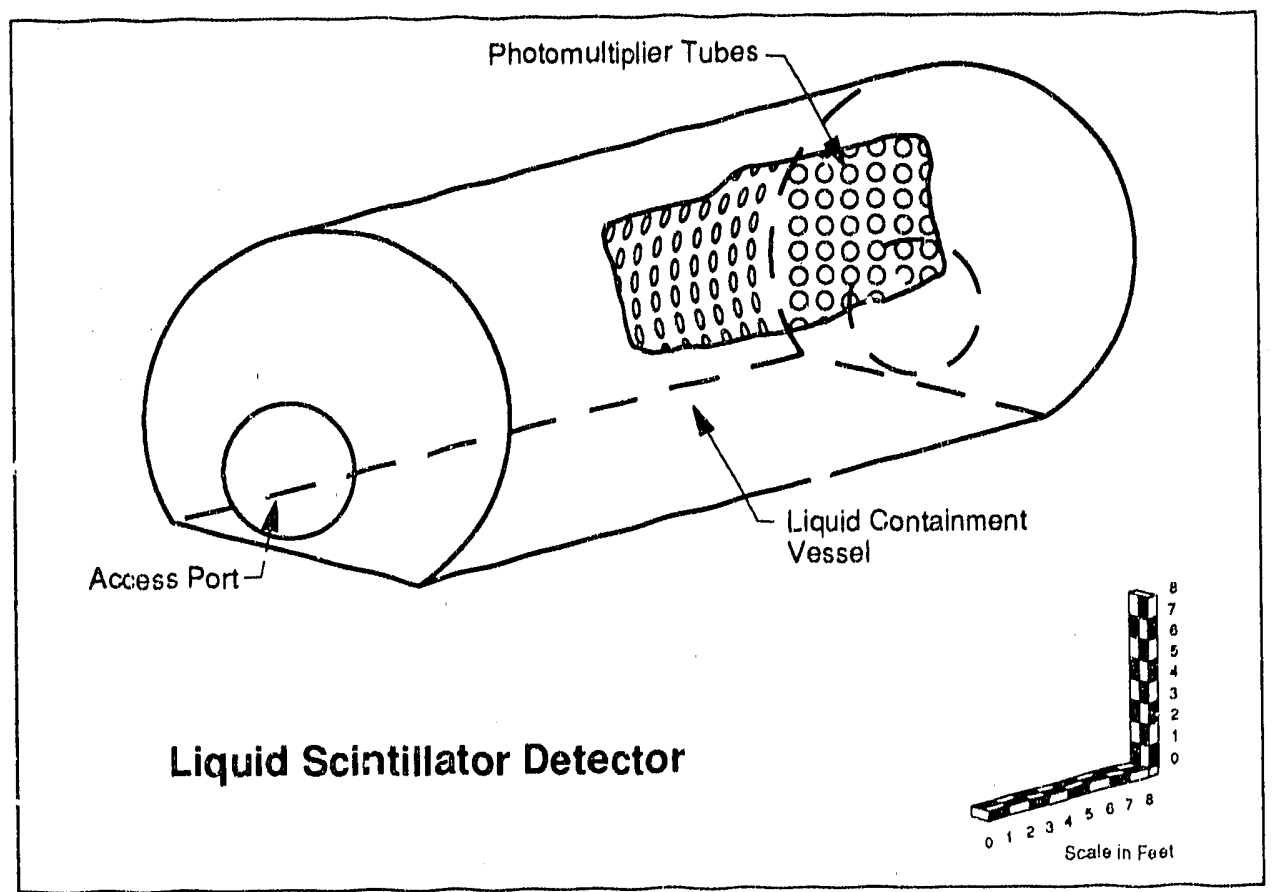

Fig. 1. A schematic view of the detector, consisting of a $6-m$ diameter by 9-m

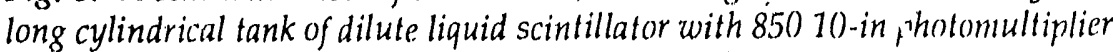
tubes covering about $28 \%$ of the surface area on the tank. 
A considerable amount of work has gone into designing and simulating the detector and understanding the backgrounds that can be expected from the experiment. The design and cost estimate are finished and the detector is scheduled for operation beginning in the summer of 1992. Fifty 10-in photomultiplier tubes have been ordered for testing, an initial design of the front-end electronics and data acquisition system has been completed, and prototyping has begun. In addition, several liquid scintillator tests are underway to ensure that Cerenkov light is being produced, to measure the time delay of the scintillation light, and to determine the optimal liquid scintillation mixture. Finally, Figs. 2 and 3 show the $\nu_{\mu} \rightarrow \nu_{e}\left(\bar{\nu}_{\mu} \rightarrow \bar{\nu}_{e}\right)$ oscillation signal (at a level of $5 \times 10^{-1}$ ) and the main backgrounds as a function of energy. Note that the backgrounds contribute only at the few times $10^{-4}$ level or smaller.

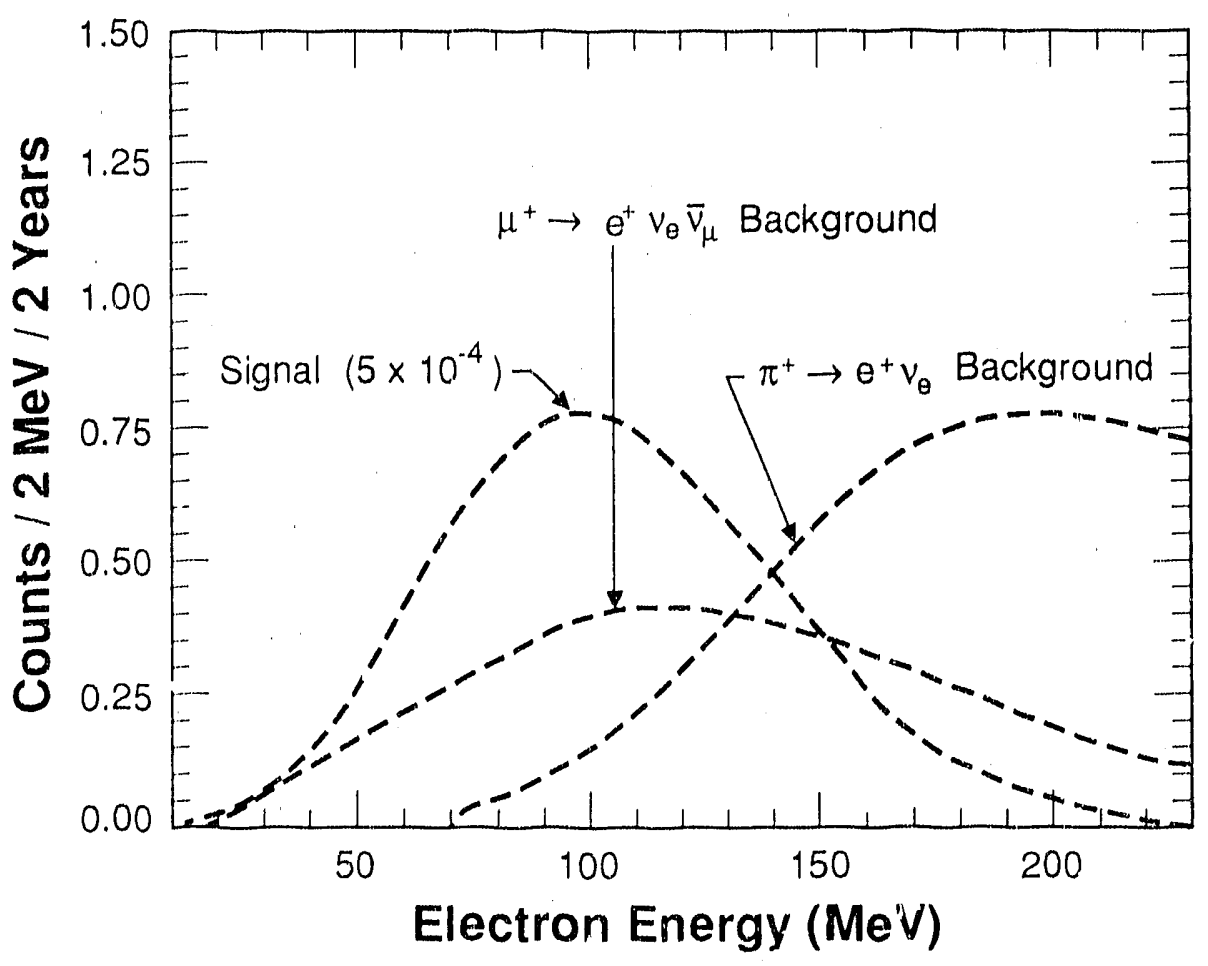

Fig. 2. The $\nu_{\mu} \rightarrow \nu_{e}$ oscillation signal (at a level of $5 \times 10^{-4}$ ) and the main backgrounds as i function of electron energy. 


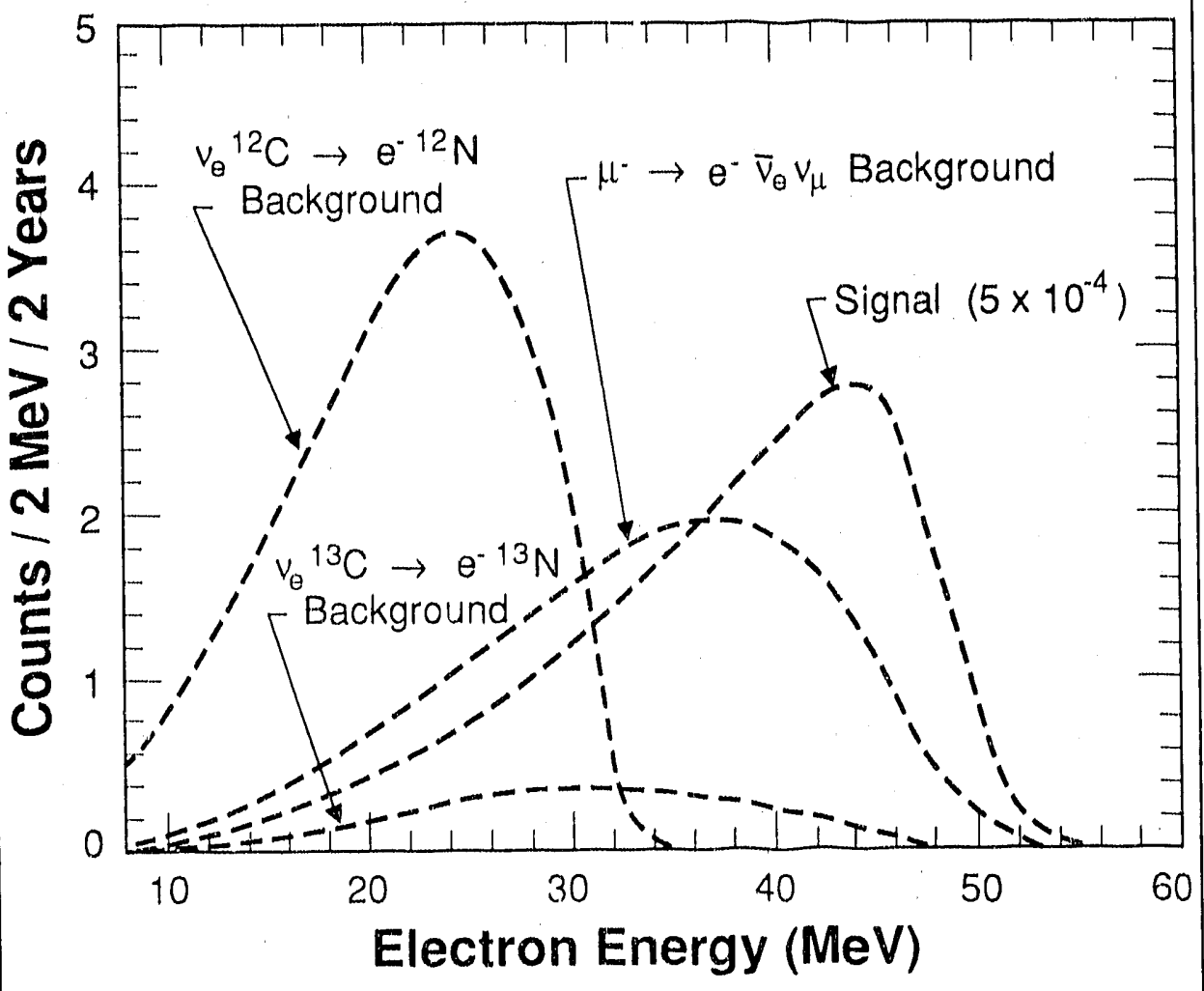

Fig. 3. The $\bar{\nu}_{\mu} \rightarrow \bar{\nu}_{e}$ oscillation signal iat a level of $5 \times 10^{-4}$ ) and the main backgrounds as a function of electron energy. 
EXPERIMENT $1123 \ldots$ NTOF

The ${ }^{16} \mathrm{O}(p, n)^{16} \mathrm{~F}$ Reaction at $500 \mathrm{MeV}$

\author{
UCLA, Los Alamos, Ohio \\ State Univ., IUCF, Rice \\ Univ., Unilv. of Colorado, \\ Ohio Univ., Loulsiana \\ State Univ.
}

Spokesman: C. A. Whitten (UCLA)

Participants: C. A. Whitten, Jr., E. Güimez, G. J. łoo, R. Byrd, A. LIng, L. Rybarcyk, T. Taddouccl, D. Marchlenskl, E. Sugarbaker, C. Goodman, W. Huang, D. Adams, $r$. Gaussiran, $D$. Nercer, D. Prout, X. Y. Chen, J. Rapaport, and A. Fazely
Experiment 1123 studied the ${ }^{16} \mathrm{O}(p, n){ }^{16 \mathrm{~F}}$ reaction at $500 \mathrm{MeV}$ with two complementary purposes in mind. From the point of view of nuclear structure physics, Gamow-Teller (CT) transition strengths were measured for a case where this strength is entirely the result of particle-hole correlations in the ground state. The target nucleus ${ }^{16} \mathrm{O}$ has $N=Z$ and is a doubly magic nucleus with completely filled $1 s$ and $1 p$ major shells in a zeroth-order shell-model picture. To this order, the summed matrix elements of the $\vec{\sigma} \cdot \vec{\tau}$ CT operator for transitions between the ${ }^{16} \mathrm{O}$ ground state and levels in ${ }^{16} \mathrm{~F}$ will be zero. However, particle-hole correlations in the ${ }^{16} \mathrm{O}$ ground-state wave function will allow GT strength to be measured in the ${ }^{16} \mathrm{O}(p, n)$ reaction using the well-established facts ${ }^{1}$ that at intermediate energies the zero-degree $(p, n)$ reaction preferentially excites transitions with large GT matrix elements and that the angular distributions for these GT transitions are strongly peaked in the forward direction with characteristic $L=$ 0 patterns.

Explicit calculations by Arima and Strottman ${ }^{2}$ and Haxton ${ }^{3}$ would indicate that the summed GT strength for ${ }^{16} \mathrm{O} \rightarrow{ }^{16} \mathrm{~F}$ transitions is $\approx 0.3-0.4$. Fazely et al. ${ }^{4}$ previously studied the ${ }^{16} \mathrm{O}(p, n)^{16} \mathrm{~F}$ reaction at 99 and $135 \mathrm{MeV}$ using the Indiana University Cyclotron Facility (IUCF) and found two $L=0$ transitions at excitation energies of 4.65 and $6.23 \mathrm{MeV}$ in ${ }^{16} \mathrm{~F}$ with GT strengths of 0.09 and 0.08 , respectively. However, this previous work did not extend to excitation energies in ${ }^{16} \mathrm{~F}$ above about $10 \mathrm{MeV}$. In the present experiment, we studied ${ }^{16} \mathrm{O}(p, n)$ transitions up to an excitation energy in ${ }^{16} \mathrm{~F}$ of at least $60 \mathrm{MeV}$.

Another important reason to study GT transitions in ${ }^{16} \mathrm{O}$ concerns the use of water Cerenkov counters to study neutrino-electron scattering. In such counters, an important source of background comes from $\nu_{e^{-16}} \mathrm{O}$ interactions,

$$
\nu_{e}+{ }^{16 \mathrm{O}} \rightarrow{ }^{16} \mathrm{~F}^{*}+e^{-} \text {, }
$$

where the electron from this reaction emits Cerenkov light in a fashion similar to the recoil electron from the $\nu_{e}-e$ elastic scattering event. A large collaboration of at least ten institutions has recently proposed to make a precise measurement of the Weinberg angle from a measurement of the ratio

$$
R=\frac{\sigma\left(\nu_{\mu} c\right)}{\sigma\left(\nu_{e} e\right)+\sigma\left(\bar{\nu}_{\mu} e\right)}
$$

using time-scparated neutrino fluxes $\left(\nu_{\mu}\right.$ from $\pi^{+} \rightarrow \mu^{+}$, and $\nu_{e}$ and $\bar{\nu}_{\mu}$ from $\left.\mu^{+}-c^{+} \nu_{e}+\bar{\nu}_{\mu}\right)$ produced by stopping tightly bunched, very intense $800-\mathrm{MeV}$ proton beams in a heavily shiclded target. "In this proposal, the energy spectrum of $\nu_{e}$ from the production target extends up to just over $50 \mathrm{McV}$, so that it is important to understand the excitation of states in ${ }^{16} \mathrm{~F}$ up to excitation energies of about $30 \mathrm{MeV}\left[Q^{1 f i} \mathrm{O}\left(\nu_{r}, r^{-}\right)=-15.43 \mathrm{MeV}\right]$.

In the data-taking runs for Exp. 1123 during the month of May 1989, the NTOF facility was opcrated with a neutron flight path of $\sim 345 \mathrm{~m}$, and a 2is micropuise spacing for the proton beam incident on target. For this flight 
palh and microstructure, the prompt gammas were separated from the highestenergy neutrons by about 370 ns, the wrap-around neutrons had kinetic energies $\leq 55 \mathrm{MeV}$, and the resolution was about $1.0 \mathrm{MeV} / \mathrm{ns}$ for $T_{n}=450 \mathrm{MeV}$. Under these conditions, ${ }^{16} \mathrm{O}(p, n)$ transitions were measured for excitation energles in ${ }^{16} \mathrm{~F}$ up to at least $65 \mathrm{MeV}$.

The oxygen targets were ice targets $1 / 8$-in thick with a diameter of $1.5 \mathrm{in}$; the material around these ice-target disks was $1 / 8$-in thick copper. In the case of the ${ }^{16} \mathrm{O}$ target, the windows were 1/4-mil aluminized mylar-thus, there was about one ${ }^{12} \mathrm{C}$ nucleus in the window for every $250{ }^{16} \mathrm{O}$ nuclei in the $1 / 8$-in thick ice target-while for the ${ }^{18} \mathrm{O}$ target $\left(95.6 \%{ }^{18} \mathrm{O}\right)$, the windows were $1 / 2$-mil thick copper. These ice targets were maintained at temperatures of -65 to $-70^{\circ} \mathrm{C}$ by a "cold-finger" type refrigeration system. No deterioration of these ice targets was observed over more than two weeks of data taking. The measurements of the target weights before and after the data taking indicate that possible changes in amount of target material were $\leq 1 \%$. Beam halo striking the $1 / 8$-in thick copper blank surrounding the ice target disk produced some background in this experiment. With careful tuning of the beam spot size, these background yields could be kept to $\leq 3 \%$ of the ice target yields. This background was measured and the beam tune was monitored by runs on a "blank" target which was identical to the ice target except for a 1.5-in. diameter hole in place of the ice and mylar windows. The ${ }^{16} \mathrm{O}$ ice target was about $95 \%$ natural water $\left(99.76 \%,{ }^{16} \mathrm{O}\right)$ and $5 \%$ water enriched in ${ }^{18} \mathrm{O}(95.6 \%)$. The ${ }^{18} \mathrm{O}(p, n)^{18} \mathrm{~F}$ reaction $[Q(p, n)=-2.437 \mathrm{MeV}]$ has a very strong GT transition to the $1^{+18} \mathrm{~F}$ ground state $[B(\mathrm{GT})=3.2]^{6}$ and the peak corresponding to this transition is woll separated from any neutron yield for the ${ }^{16} \mathrm{O}(p, n)^{16} \mathrm{~F}$ reaction $(Q=-16.21 \mathrm{MeV})$. This peak therefore served two purposes:

1. It provided a quick check for the operation of the complete NTOF dataacquisition system, and

2. It provided a normalization peak if the target was sublimating during the data-taking period.

Data were taken for the $500-\mathrm{MeV}{ }^{16} \mathrm{O}(p, n)^{16} \mathrm{~F}$ reaction at laboratory angles of $0,1,2,3,4,6$ and 8 degrees. These angles were chosen to provide accurate characterizations of both the forward-peaked, $L=0$, GT transitions, which fall off sharply with increasing angle and the $L \geq 1$ transitions, which peak at nonzero laboratory angles. At each angle setting the total data accumulated for the ${ }^{16} \mathrm{O}$ ice target was greater than $2 \times 10^{6}$ events, except for the case of $\theta_{\text {lab }}=3^{\circ}$, where we obtained about $1 \times 10^{6}$ events. For each angle, data runs were also taken for the ${ }^{18} \mathrm{O}$ ice target, the blank target frame, a 27.5-mil thick copper target, a target made up of 50 layers of $1 / 4$-mil aluminized mylar, a thick ${ }^{7} \mathrm{Li}$ target, and a ${ }^{13} \mathrm{C}$ target. Most of these runs on targets other than the ${ }^{16} \mathrm{O}$ ice target were to provide data for the necessary background subtractions on the raw ${ }^{16} \mathrm{O}$ ice target data and to monitor the beam halo. The data from the ${ }^{7} \mathrm{Li}$ target provided an absolute normalization for the $(p, n)$ cross sections. At a given angle setting, 
RESEARCH

Nuclear and Particle Physics

runs on the various largets were interspersed. Typically, there were about 35 runs at each angle.

Figures 1 and 2 present $(p, n)$ data from individual runs $(\sim 1 / 10$ to $1 / 15$ of the total data) on the ${ }^{16} \mathrm{O}$ ice target at the seven laboratory angles measured in this experiment. At forward angles, a peak corresponding to the strong ${ }^{18} \mathrm{O}(p, n){ }^{18} \mathrm{~F}$ ground state GT transition ( $L=0)$ stands out in the spectra, and at all angles one observes two peaks from ${ }^{16} \mathrm{O}(p, n){ }^{16} \mathrm{~F}$ transitions. The peak width (FWHM) of slightly more than $1 \mathrm{MeV}$ is to be expected from a convolution of contributions from the target thickness $(0.77 \mathrm{MeV})$, the neutron detector thickness, the intrinsic timing, and the rebunching of the bearn micropulse. From the work of Fazely et al., ${ }^{4}$ these two peaks correspond to $\mathrm{L} \geq 1{ }^{16} \mathrm{O}(p, n)$ transitions for a 2- -3 - doublet in ${ }^{16} \mathrm{~F}$ at 0.43 and $0.72 \mathrm{MeV}$, respectively, and for a $2-$ level at $7.6 \mathrm{MeV}$. From these raw spectra, one can qualitatively search for $L=0 \mathrm{GT}$ strength by observing in which regions of excitation energy there is a fall-off of yield with increasing laboratory angle. These data would then indicate definite GT strength for ${ }^{16} \mathrm{~F}$ states at excitation energies between 3.5 and $16.5 \mathrm{MeV}$-in agreement with the results of Fazely et al. ${ }^{4}$ - and probably some strength between about $8 \mathrm{MeV}$ and $15 \mathrm{MeV}$. Of course, quantitative results for the ${ }^{16} \mathrm{O}(p, n)^{16} \mathrm{~F}$ GT strength observed in this experiment will only be obtained after careful analyses of these data sets. We will search for GT strength and set upper limits on possible GT strength by studying the final (background subtracted) ${ }^{16} \mathrm{O}(p, n)$ angular distributions as a function of excitation energy in ${ }^{16} \mathrm{~F}$, using excitation energy bins of about $1 \mathrm{MeV}$, commensurate with our experimental resolution. Replay of the data tapes is now in progress.

\section{References}

1. T. N. Taddeucci, C. A. Goulding, T. A. Carey, R. C. Byrd, C. D. Goodman, C. Gaarde et al., Nucl. Phys. A 469, 125 (1987).

2. A. Arima and D. Strottman, Phys. Lett. 96B, 23 (1980).

3. W. C. Haxton, Phys. Rev. D 36, 2283 (1987); W' C. Haxton, Phys. Rev. C 37, 2660 (1988).

4. A. Fazely, B. D. Anderson, M. Ahmad, A. R. Baldwin, A. M. Kalenda, R. J. McCarthy et al., Phys. Rev. C 25, 1760 (1982).

5. R. C. Allen, X-Q. Lu, K. Gollwitzer, G. J. Igo, E. Gulmez, C. Whitten et al., "A Proposal for a Precision Test of the Standard Model by NeutrinoElectron Scattering," Los Alamos National Laboratory report LA-11300-P (April 1988).

6. B. D. Anderson, A. Fazely, R. J. McCarthy, P. C. Tandy, J. W. Watson, R. Madey et al., Phys. Rev. C27, 1387 (1983). 
RESEARCH

Nuclear and Particle Physics

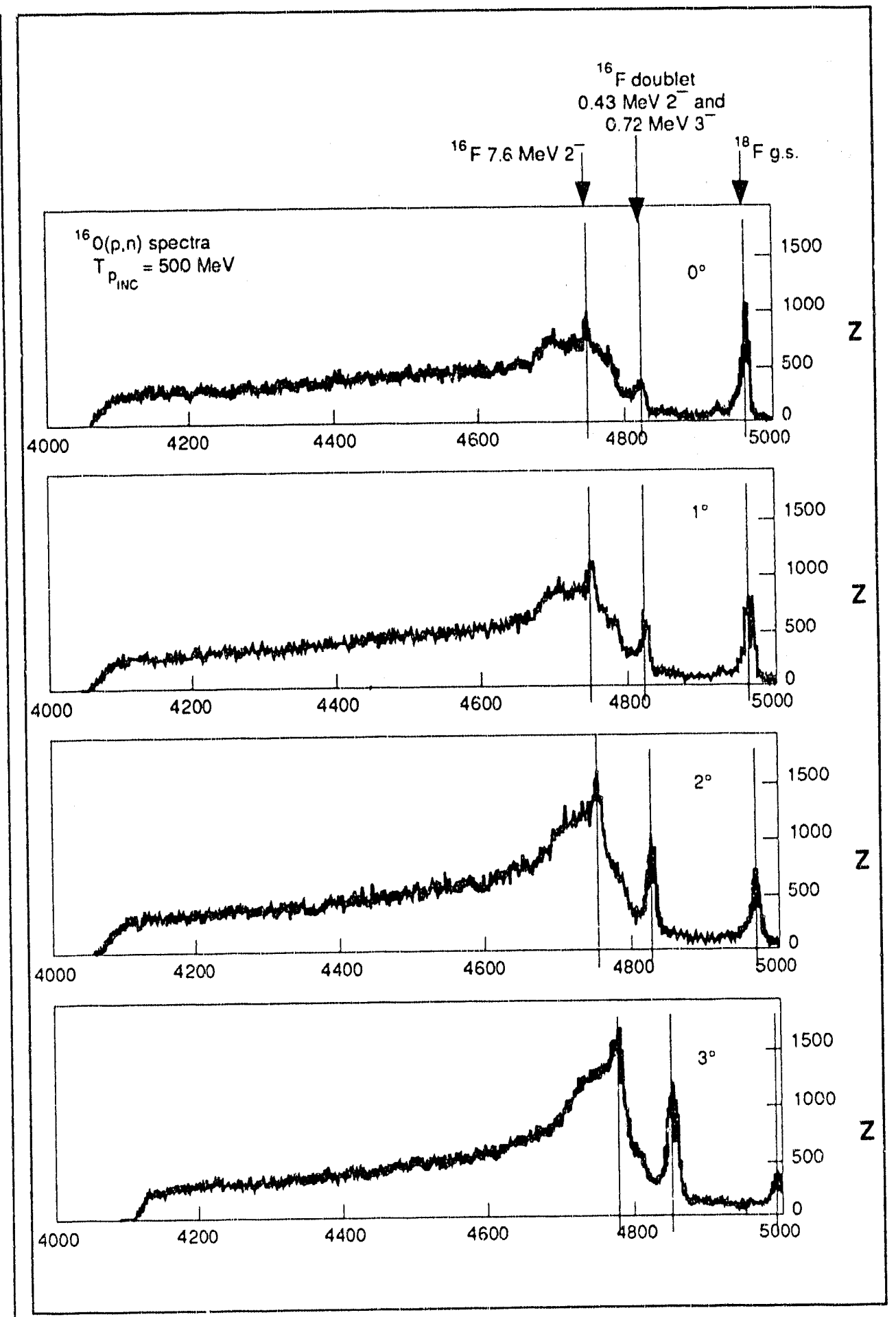

Fig. 1. On-line data for ${ }^{16} \mathrm{O}$ ice target. 


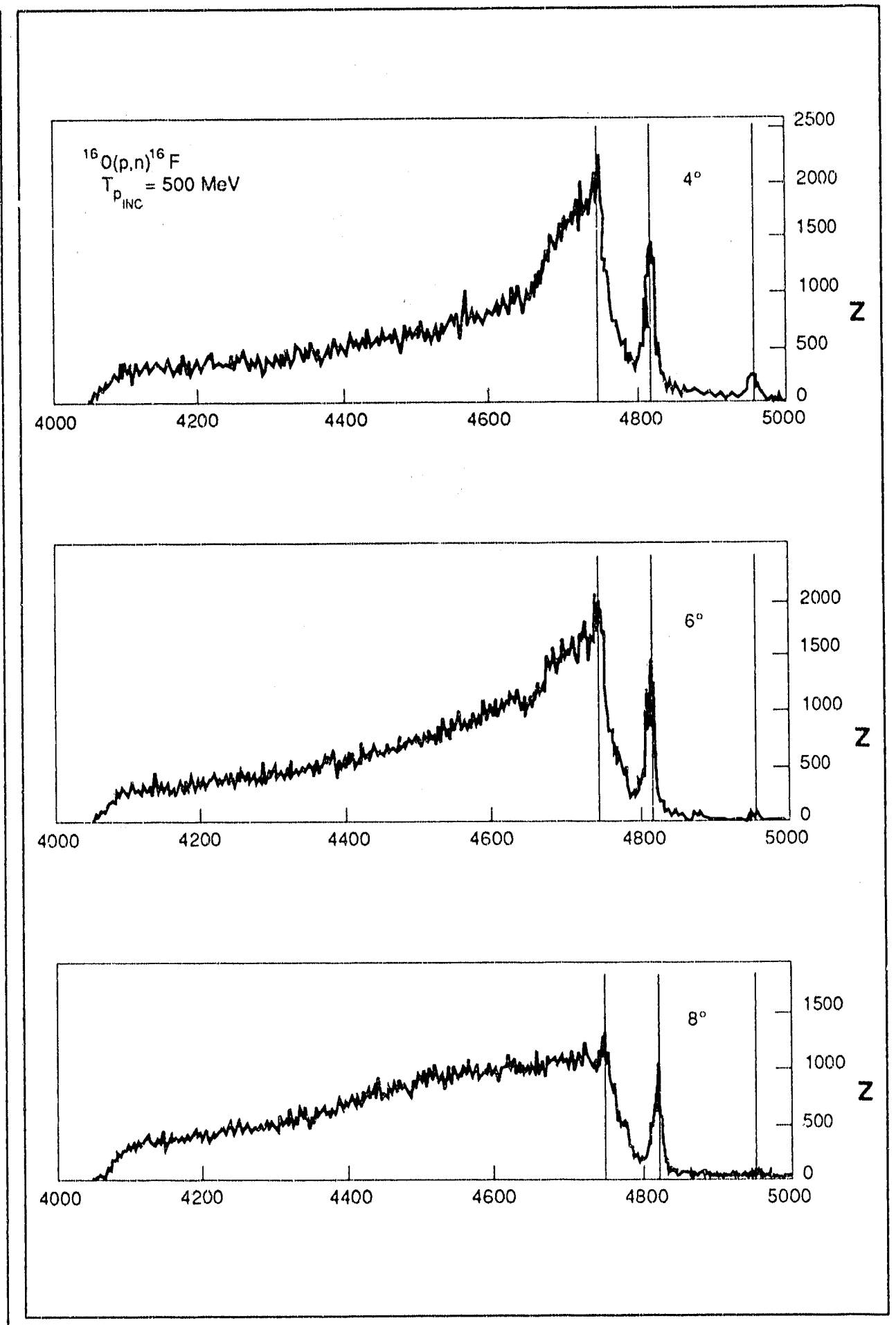

Fig. 2. On-line data for ${ }^{16} \mathrm{O}$ ice target. 
EXPERIMENT 750 - P3 . East

Reaction Mechanism of Inclusive Pion Double Charge Exchange in ${ }^{4} \mathrm{He}$

\author{
MIT, Los Alamos, Univ. \\ of Wyoming, Univ. of \\ Michigan, Univ. of Illinois \\ Participants: E. R. Kinney, J. L. \\ Matthews, P. A. M. Gram, G. A. \\ Rebka, Jr, D. A. Roberts, and \\ S. A. Wood
}

Inclusive pion double charge exchange (DCX) has been under investigation in a series of experiments conducted at LAMPF since 1981. ${ }^{1-6}$ This necessarily multiple-scattering process has been observed in ten nuclei ranging in mass number $A$ from 3 to 208, with both $\pi^{+}$and $\pi^{-}$beams ranging in energy from 120 to $270 \mathrm{McV}$. Two interesting phenomena appeared in these measurements:

1. For the very light nuclei ${ }^{3,4} \mathrm{He}$, the outgoing pion spectra deviated markedly from the shape of four-body (or any other) phase space. ${ }^{2,4,7}$ The spectra at small angles contained a prominent peak at high energy, which decreased in height and moved to lower energy with increasing angle.

2. For heavier nuclei, the $A$-dependences of the $\left(\pi^{+}, \pi^{-}\right)$and $\left(\pi^{-}, \pi^{+}\right)$cross sections were found to be strikingly different; whereas the former increases more " less morotonically with $A$, the latter appears to "saturate" at $A=40$ (Ref. 8).

It turns out that both of these empirical observations can be accounted for by the same simple model of $\mathrm{DCX}$, in which the reaction proceeds by two sequential single charge exchanges (SCX) (see Fig. 1), provided that some account is taken of the other nucleons in the nucleus. An empirical scaling rule has been discovered that explains the different $A$-dependences of the $\left(\pi^{+}, \pi^{-}\right)$and $\left(\pi^{-}, \pi^{+}\right)$reactions. ${ }^{8}$ Here we present an explanation of the shape of the doubly differential cross section for DCX in ${ }^{4} \mathrm{He}$.

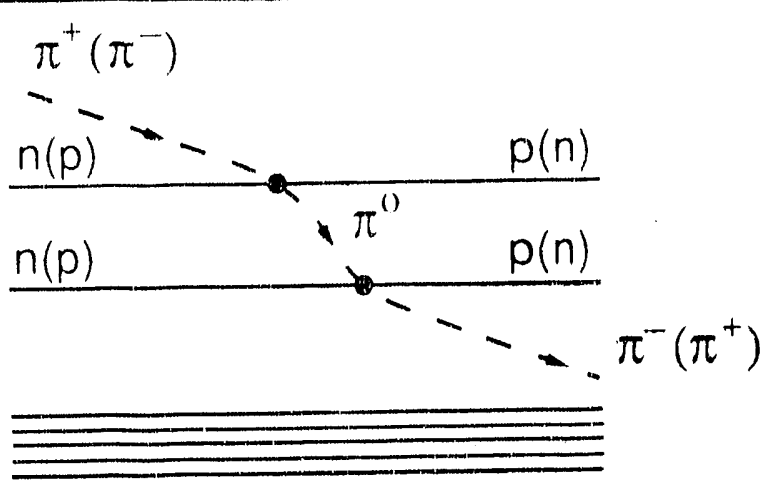

Fig. 1. Sequential single-charge-exchange mechanism for double charge exchange.

The first of these was proposed by Wood, ${ }^{1}$ based on the sequential SCX picture of DCX (Fig. 1). In the $\Delta(1232)$ resonance region, the pion-nucleon interaction is predominantly $p$-wave, and thus, the SCXs have a $\left(1+3 \cos ^{2} \theta\right)$ angular distribution. Forward and backward SCXs are thus enhanced relative to reactions at intermediate angles. In DCX, a pion detected at a forward angle can have suffered two small-angle scatterings, resulting in small energy loss, 
or two large-angle scatterings, resulting in large energy loss, or of course, two intermediate-angle scatterings, with corresponding intermediate energy losses. However, since the first two cases are more probable than the third, one might expect the forward-angle DCX cross section to be enhanced at large and at small outgoing pion energies. Wood ${ }^{1}$ calculated the energy spectra of the outgoing pions in DCX, assuming the reaction to proceed through two SCXs on nucleons in a Fermi gas. Using the free pion-nucleon angular distribution for the $\mathrm{SCX}$, a double-peaked spectrum in DCX at forward angles was indeed seen. When an isotropic SCX angular distribution was used instead, the DCX spectrum became single-peaked. Calculations were performed for DCX in ${ }^{4} \mathrm{He}$ by Thies and van Loon, ${ }^{9}$ which yielded two peaks in the spectra at forward angles but which did not resemble the data. It was not until the calculation of Kinney, ${ }^{2}$ which took account of some of the effects of the nuclear medium in which the SCXs were occurring, that reasonable quantitative agreement with the shapes of the ${ }^{4} \mathrm{He}$ energy spectra was achieved.

These calculations used a semiclassical model of pion double scattering based on a formalism developed by Thies ${ }^{10}$ to describe inclastic multistep reactions with quantum-mechanical transport theory. The pion-nucleus interaction, $t$-matrix $T_{A}$, is expanded in the multiple scattering series

$$
T_{A}=\sum_{i=1}^{A} \tau_{i}+\sum_{i \neq j=1}^{A} \tau_{i} g \tau_{i}+\ldots,
$$

where $g=1 /\left(E-T_{\pi}-H_{A}\right)$ describes the propagation through the nuclear medium, and the in-medium pion-nucleon $t$ matrix $\tau_{i}=v_{i}+v_{i} g \tau_{i}\left(v_{i}=\pi N\right.$ potential) describes the interaction between the pion and bound nucleon $i$. The first term on the right-hand side of Eq. (1) is zero to first order for DCX. Terms representing triple- and higher-order scattering are ignored. To further simplify the calculation, Kinney used plane-wave impulse approximation, i.c., neglected distortions of the pion waves and final-state interactions among the nucleons, and replaced $\tau_{i}$ by the free $\pi N t$-matrix $t_{i}$. However, a nonstatic prescription was used for the energies at which $t_{i}$ and $t_{j}$ [replacing $\tau_{i}$ and $\tau_{j}$ in Eq. (1)] are evaluated. This method takes account of Fermi motion and of the energy dependence of the $\pi N$ interaction, which is an important effect near the $\Delta$ (1232) resonance. (The pions will prefer to interact with those nucleons in the nucleus whose velocity is such that the relative $\pi N$ energy is near that of the $\Delta$.) Pauli blocking is also included.

Owing to the neglect of distortions and final-state interactions, one might not expect to be able to predict the absolute magnitude of the DCX cross section, but one might hope to reproduce the shape of the forward-angle spectrum. The results of the calculation for the doubly differential cross section for the ${ }^{4} \mathrm{He}\left(\pi^{+}, \pi^{-}\right) 4 p$ reaction at $T_{\mathrm{inc}}=240 \mathrm{McV}$ and $\theta=25^{\circ}$ are shown in Fig. 2 . The magnitude of the predicted cross section is perhaps surprisingly close to that measured. The different curves represent different assumptions as to the depth 


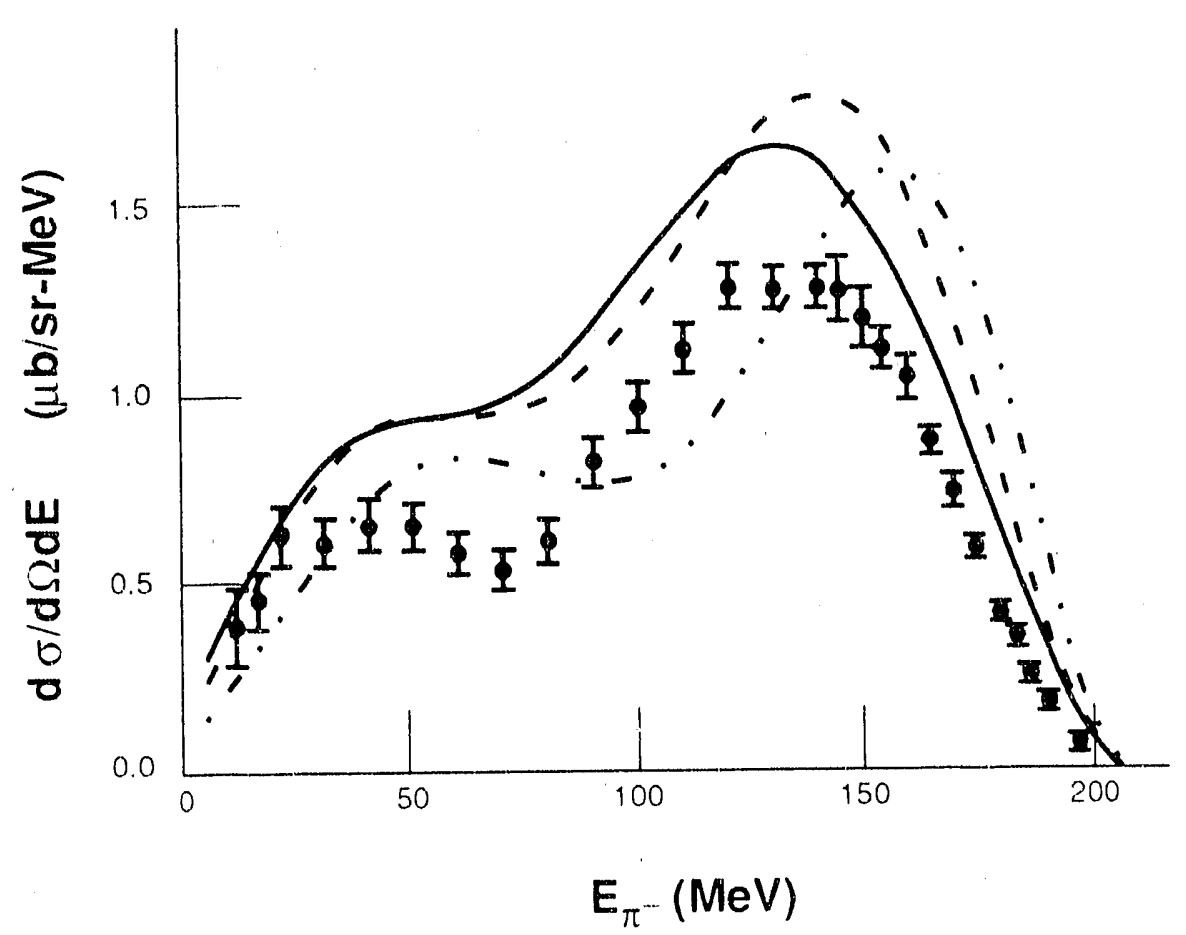

Fig. 2. The doubly differential cross section for the ${ }^{4} \mathrm{He}\left(\pi^{+}, \pi^{-}\right) 4 p$ reaction at $240-\mathrm{MeV}$ incident pion energy and $25^{\circ}$ laboratory angle. The curv. - represent the cross section calculated with different values of the average nuclear potential: $U_{2}=U_{2}=-55 \mathrm{MeV}$ (solid), $U_{1}=U_{2}=-36.8 \mathrm{MeV}$ (dashed), and $U_{1}=U_{2}=0$ (dot-dashed).

of the nuclear potential in which the two SCX interactions take place. Taking $U_{1}=U_{2}=-55 \mathrm{MeV}$ best fits the position of the high-energy peak. The implication of this assumption is that both interactions occur in the central region of the shell-model potential well.

As seen in Fig. 3, the calculation overestimates the measurements at lower energies; this is probably a symptom of the importance of distorted waves and final-state interactions. Figure 4 illustrates that the calculation is nonetheless able to predict the shape of the $25^{\circ}$ spectra over the range of incident energies at which measurements were made. We believe that the most important conclusion of this work is that the double-peaked spectrum observed at forward angles, unexpected experimentally, is consistent with a sequential SCX picture, without invocation of more exotic reaction mechanisms, or of more mundane interactions among the nucleons in the final state. However, reproducing the absolute magnitude of this cross section, as well as its complete incident-energy and angular dependence, may well require inclusion of these other processes. 


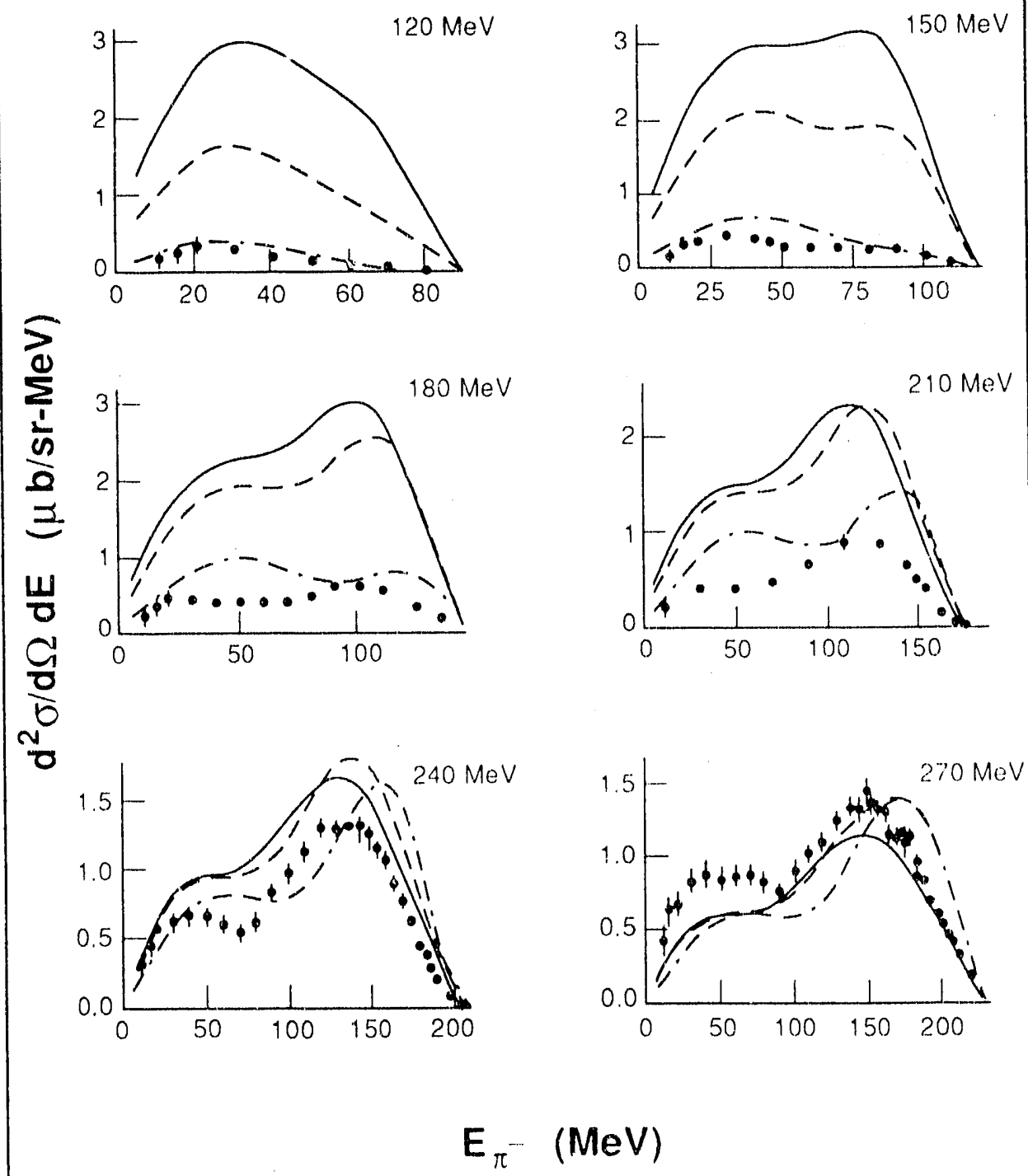

Fig. 3. The doubly differential cross section for the ${ }^{4} \mathrm{He}\left(\pi^{+}, \pi^{-}\right) 4 p$ reaction at $25^{\circ}$ laboratory scattering angles and incident prion energies of 120,150, 180, 210, 240, and 270 MeV. The different curves correspond to different values of the average nuclear potential: $U_{1}=U_{2}=-55 \mathrm{MeV}$ (solid), $U_{1}=U_{2}=$ $-36.8 \mathrm{MeV}$ (dashed), and $U_{1}=U_{2}=0$ (dot-dashed). 


\section{RESEARCH}

Nuclear and Particle Physics
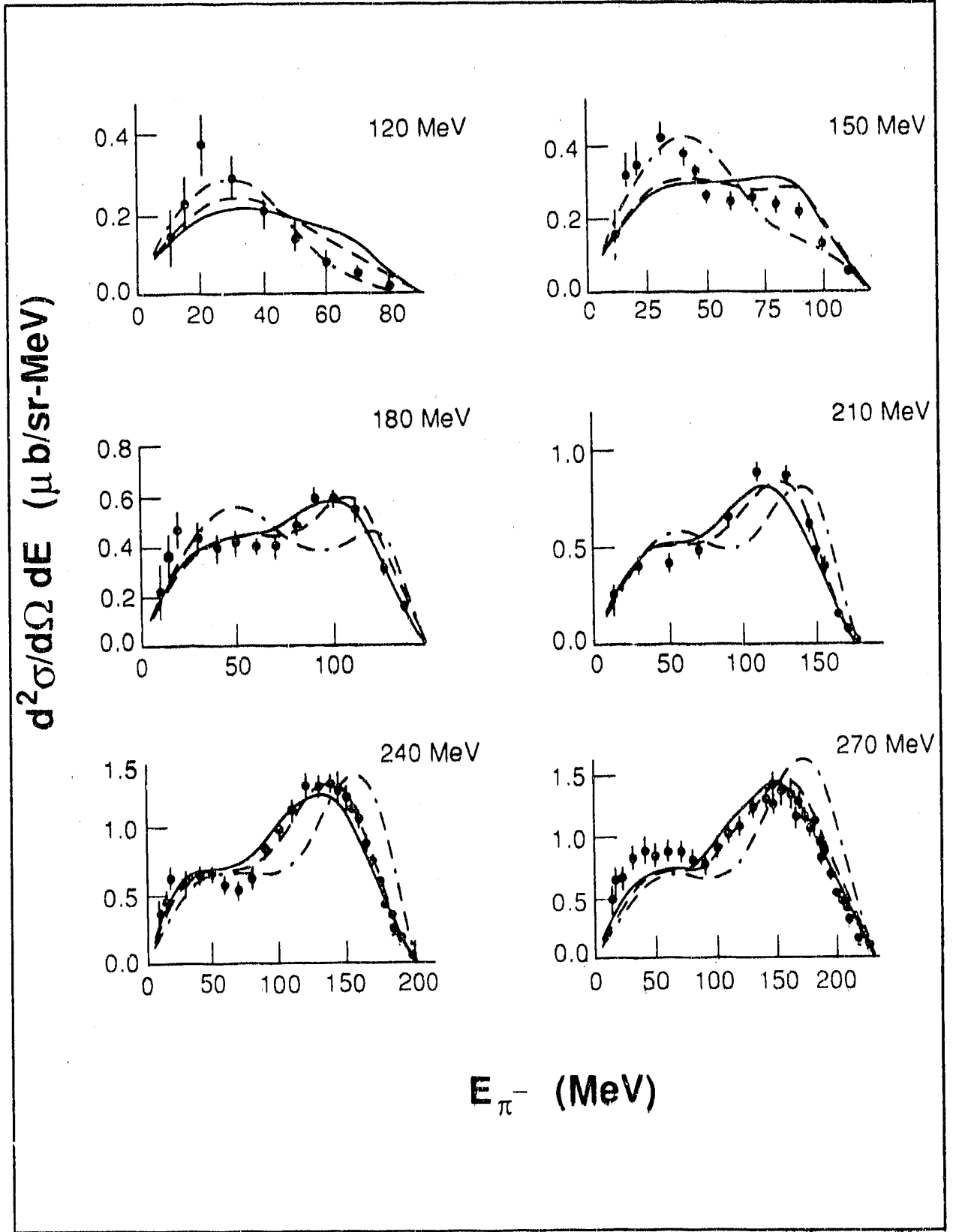

Fig: 4. The doubly differential cross section for the ${ }^{A} \mathrm{He}\left(\pi^{+}, \pi^{-}\right) 4 \mathrm{p}$ reaction at $25^{\circ}$ laboratory scattering angle and incident pian energies of $120,150,180$, 210, 240, and 270 MeV. The different curpes correspand to different values of the average nuflear potential: $U_{1}=U_{3}=-55 \mathrm{MeV}$ (solid), $U_{1}=U_{2}=$ $-36.8 \mathrm{MeV}$ (dashed), and $U_{1}=U_{2}=0$ (dat-dashed). All of the theoretical predictions have been renormalized to yield the same integrated area as that of the measured cross sections. 


\section{References}

1. S. A. Wood, Ph.D. thesis, Massachusetts Institute of Technology, Los Alamos National Laboratory report LA-9932-T (1984).

2. E. R. Kinncy, Ph.D. thesis, Massachusetts Institute of Technology, Los Alamos National Laboratory report LA-11417-T (1988).

3. S. A. Wood, J. L. Matthews, G. A. Rebka, Jr., P. A. M. Gram, H. J. Ziock, D. A. Clark et al., Phys. Rev. Lett. 54, 635 (1985).

4. E. R. Kinney, J. L. Matthews, P. A. M. Gram, D. W. MacArthur, E. Piasetzky, G. A. Rebka, Jr., and D. A. Roberts, Phys. Rev. Lett. 57, 3152 (1986).

5. J. L. Matthews, in Proceedings of the LAMPF Workshop on Pion Double Charge Exchange, Los Alamos National Laboratory report LA-105550-C (1985), p. 275.

6. P. A. M. Gram, in Pion-Nucleus Physics: Future Directions and New Facilities at LAMPF, R. J. Peterson and D. D. Strottman, Eds. [AIP Conf. Proc. 163, 79 (1988)].

7. M. Y. D. Wang, S.B. thesis, Massachusetts Institute of Technology, 1987 (unpublished).

8. P. A. M. Gram, S. A. Wood, E. R. Kinney, S. Hoibraten, P. Mansky, J. L. Matthews ct al., Phys. Rev. Lett. 62, 1837 (1989).

9. J. van Loon, M.S. thesis, Free University of Amsterdam, 1985 (unpublished). Translation contained in Ref. 2.

10. M. Thies, Ann. Phys. (N.Y.) 123, 411 (1979). 
EXPERIMENT $1103-\mathrm{p}^{3}$. East

\section{Inelastic Pion Scattering on ${ }^{18} 0$ Above the $\Delta$ Resonance}

\author{
Los Alamos, Univ. of Texas \\ at Austin, Rutgers Univ. \\ Spokesmen: A. L. Williams and \\ C. F. Moore (Univ. of Toxas at \\ Austin) \\ Participants: K. W. Johnson, \\ M. Jones, J. L. McDonald, \\ C. F. Moore, C. L. Morris, \\ S. J. Seestrom, A. L. Williams, \\ D. L. Hilliams, and S. H. Yoo
}

In Exp. 1103, inclastic pion scattering has been measured on ${ }^{18} \mathrm{O}$ for pion kinetic energies above $300 \mathrm{MeV}$ using the Large Acceptance Spectrometer (LAS) and the $P^{3}$ channel. Angular distributions were taken at 400 and $500 \mathrm{MeV}$ for $15^{\circ} \leq \theta_{\text {lab }} \leq 40^{\circ}$ and single angles were measured at $300,425,450,475$, and $525 \mathrm{MeV}$. At present, the angular distributions are still in analysis, but the other measurements are in a preliminary state and are reported here.

The experiment was motivated by the expectation that at higher energies some of the dominant processes in the $\Delta$ resonance region will be minimized. Around $500 \mathrm{MeV}$, new resonances appear that are dominated by $T=1 / 2$ amplitudes, and new bel:avior might be seen for states dominated by pure neutron or proton transitions. For ${ }^{18} \mathrm{O}$, large differences were expected between $\pi^{+}$and $\pi^{-}$ scattering to the $2_{1}^{+}$state because of the assumption that the two valence neutrons are in the $(2 s 1 d)$ shell coupled to a closed-shell core. Therefore, only pure neutron transitions should excite the state. In a recent measurement at EPICS, ${ }^{1}$ the ratio $\sigma\left(\pi^{-}\right) / \sigma\left(\pi^{+}\right) \equiv R$ was measured to be $2.10 \pm 0.16$ at $T_{\pi}=164 \mathrm{MeV}$ and $\theta_{\text {lab }}=30^{\circ}$, which is less than the expected ratio of 9:1, as predicted by $\pi-N$ scattering at the $\Delta(1232)$ resonance for a pure neutron state. This reflects the collective nature of this low-lying $2^{+}$state. Since this state has a strong isovector component, the expected ratio should change from 9:1 to $\sim 1: 2$ as the $\Delta(1232)$ resonance is passed and new resonances are approached. While observing the actual ratio 1:2 is not assumed, a reversal in the enhancement to $\pi^{+}$might be observed.

Our preliminary results are quite interesting. We present in Fig. 1, cross sections for the $2_{1}^{+}(1.98-\mathrm{MeV})$ state along with the ratio $\sigma\left(\pi^{-}\right) / \sigma\left(\pi^{+}\right)$. Also shown are measurements from previous work. ${ }^{1,2,3}$ The cross sections reported are for $\theta_{\text {lab }}=38,30,28,27,26$, and $25^{\circ}$ at $300,400,425,450,475$, and $525 \mathrm{MeV}$, respectively. In Fig. 2, we present $\left(\sigma^{-}-\sigma^{+}\right) /\left(\sigma^{-}+\sigma^{+}\right)$as a function of energy. Since the energy resolution was about $1.9 \mathrm{MeV}$, these measurements were obtained by two methods. First, cross sections were extracted by fitting peaks to the data using peak shapes obtained from elastic scattering on ${ }^{16} \mathrm{O}$. The second method consisted of subtracting the spectrum for $\pi^{+}$from the spectrum for $\pi^{-}$ and getting the difference for the state. Then the two spectra were added, and the sum for the state was extracted using peak fitting. As can be seen in Fig. 2, the results from both methods are consistent. The expected reversal is seen at about $425 \mathrm{MeV}$, but the transition is rather sharp. If the reversal is caused by coupling to the $N(1440)$ resonance, the reversal might be expected to be strongest at $380 \mathrm{MeV}$. Since the $\Delta(1232)$ tail is still strong at this energy, that could account for the shift to $425 \mathrm{MeV}$. The abruptness of the transition is quite unexpected and puzzling, as is the apparent peak at $400 \mathrm{MeV}$ in $\pi^{-}$enhancement. We are currently trying to rule out experimental or analysis problems that could be the cause. The analysis of the 'xll angular distribution at $400 \mathrm{McV}$ will hopefully clarify the situation. If the result is correct, some interesting new physics may be the explanation. 


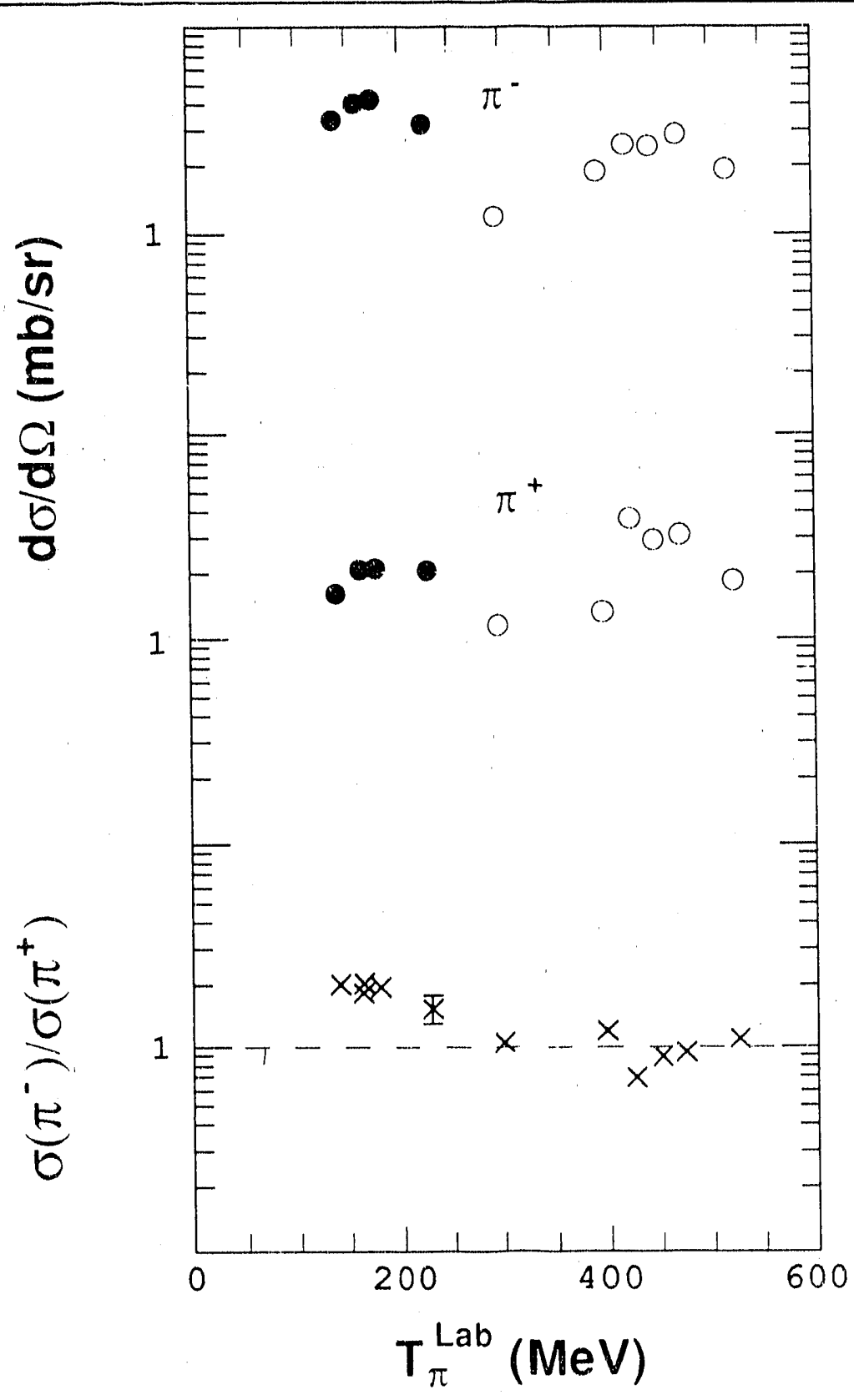

Fig. 1. Cross sections for the $2_{1}^{+}(1.98-\mathrm{MeV})$ state in ${ }^{18} \mathrm{O}$ (for various scattering angles) as a function of energy. The cross sections reported are for $0_{\text {lab }}$ $=38,30,28,27,26$, and $25^{\circ}$ at 300,400, 425, 450, 475, and $525 \mathrm{MeV}$, respectively. The filled circles represent previous work and the open circles represent new results from this work. Also shown is the ratio $\sigma\left(\pi^{-}\right) / \sigma\left(\pi^{+}\right)$, represented by $X$ 's, for each pair of cross sections at a given energy. 


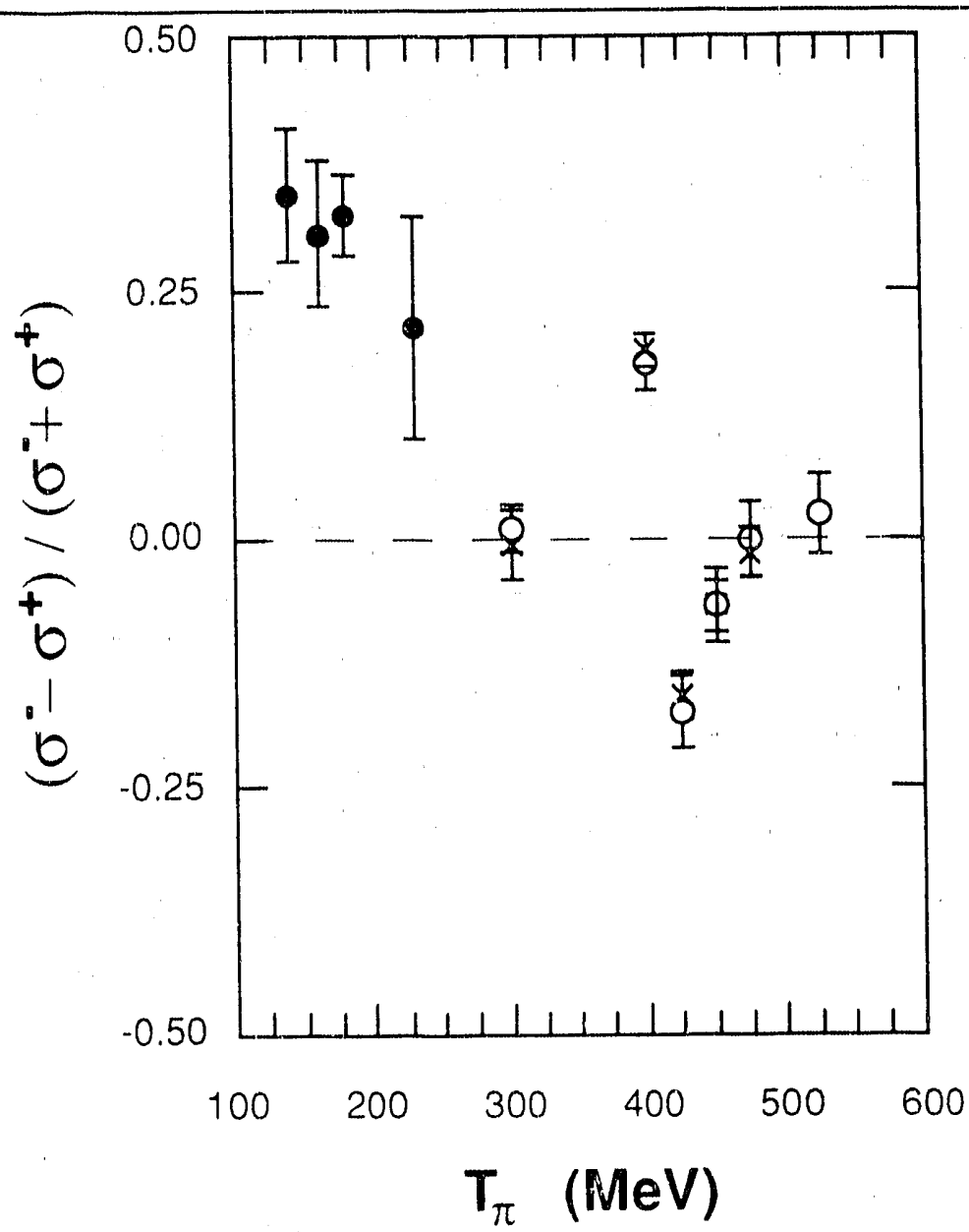

Fig. 2. The asymmetry between $\pi^{-}$and $\pi^{+}$cross sections for the $2_{1}^{+}(1.98-$ $\mathrm{MeV})$ state in ${ }^{18} \mathrm{O}$ represented by $\left(\sigma^{-}-\sigma^{+}\right) /\left(\sigma^{-}+\sigma^{+}\right)$as a function of energy. The filled circles are from previous work and the open circles and the X's represent new results from this work. The open circles are from peak fitting arld the $X^{\prime}$ 's are results from specira subtraction.

\section{References}

1. S. J. Scestrom-Morris, D. Dehnhard, M. A. Franey, D. B. Holtkamp, C. L. Blilie, C. L. Morris, J. D. Zumbro, and H. T. Fortune, Phys. Rev. C 37, 2057 (1988).

2. S. Iverson, A. Obst, Kamal K. Seth, H. A. Thiessen, C. L. Morris, N. Tanaka et al., Phys. Rev. Lett. 40, 17 (1978); S. Iverson, H. Nann, A. Obst, Kamal K. Seth, N. Tanaka, C. L. Morris et al., Phys. Lett. 82B, 51 (1979). 
3. C. L. Morris, Los Alarnos National Laboratory, Los Alamos, New Mexico, private communication (1989). 
EXPERIMENT 1107 P3. East

\section{Studies of Pion Double Charge Exchange Scattering at Energies Above the $\Delta$ Resonanse}

\author{
Los Alamos, New Mexicn \\ Siate Univ., Unir. of \\ Texas at Austin, Univ. \\ of Tennsylvania, Univ. of \\ Colorado \\ Spokesman: G. R. Burlesion (New \\ Mexico State Univ.) \\ Participants: M. Burrein, G. R. \\ Burles on, $H$. T. Fortune, d. \\ Faucell, E. Insko, F. Nie, \\ K. W. Johnson, G. P. \\ Kahrimanis, J. A. McGill, \\ C. F. Moore, C. L. Morris, \\ D. S. Oakley, J. M O'Donnell, \\ D. Smith, H. Ward, and A. L. \\ Williams
}

This experiment continues the recently initiated program of measurements of high-energy pion-nucleus double-charge-exchange scattering (DCX), which is being carricd out in the $P^{3}$ Channel with the Large Acceptance Spectrometer (LAS). A.t present, this system provides the only facility for DCX measure nents for $300-550-\mathrm{MeV}$ incident pior kinetic energies. Measurements have been made of cross sections for $\left(\pi^{4}, \pi^{-}\right)$scattering at several energies in this range on ${ }^{26} \mathrm{Mg}$, ${ }^{42,44,48} \mathrm{Ca},{ }^{46,50} \mathrm{Tt},{ }^{52} \mathrm{Cr},{ }^{54,56} \mathrm{Fe},{ }^{80} \mathrm{Se},{ }^{90} \mathrm{Zr},{ }^{197} \mathrm{Au}$, and ${ }^{208} \mathrm{~Pb}$. These include transitions to double-isobaric-analog states (DIAS) as well as nonanalog transitions to residual ground statis for $T>1$ nuclei. For many ground-state transitions, only upper limits have been sound.

Cross sections for DIAS transitions in ${ }^{42,44,4 \pi} \mathrm{Ca}\left(\pi^{+}, \pi^{-}\right)^{42,44,48} \mathrm{Ti}$ at $\theta_{\text {lat }}=$ $5^{\circ}$ are presented in Fig. 1. The data are characterized by a relatively flat energy dependence, which is consistent with the behavior previously observed ${ }^{1}$ for ${ }^{i} \mathrm{C}$ and ${ }^{17} \mathrm{O}$, as well as for other nuclei, in this energy range. At $500 \mathrm{MeV}$, $5^{\circ}$ cross sections were measured for DIAS transitions in ${ }^{26} \mathrm{Mg},{ }^{56} \mathrm{Fe},{ }^{90} \mathrm{Zr},{ }^{197} \mathrm{Au}$, and ${ }^{20 \times} \mathrm{Pb}$. These, along with those for ${ }^{14} \mathrm{C},{ }^{18} \mathrm{O}$, and ${ }^{42,44,4 \times} \mathrm{Ca}$ at $500 \mathrm{MeV}$, are shown in Fig. 2. This figure also displays $450-\mathrm{MeV}$ cross sections for ${ }^{46,50} \mathrm{Ti}$, ${ }^{52} \mathrm{Cr}$, and ${ }^{54} \mathrm{Fe}$, which are included under the assumption that the cross sections for these nuclei are also relatively flat between 400 and $500 \mathrm{MeV}$. These data are presented as the cross section $d \sigma / d \Omega$ divided by $(N-Z)(N-Z-1)$ as a function of mass number $A$, where $(N-Z)(N-Z-1)$ represents the product of the number of neutrons available for sequential single-charge-exchange scattering. The behavior of these cross sections can be well described by an $A^{-7 / 3}$ dependence, as shown by the solid lines in Fig. 2. For energies ranging between 100 and $300 \mathrm{MeV}$, an overall $A^{-10 / 3}$ dependence was previnusly found, with the $T=$ : targets appearing to have an $A^{-7 / 3}$ dependence when taken alone. ${ }^{2}$ The lowered A-dependence at higher energies suggests a weakening of the strength of the DCX interaction, as might be expected from the smaller pion-nucleon total cross sections here.

Cross sections for the seven $f_{i / 2}$-shell nuclei have been compared to a twoamplitude model, ${ }^{3,4}$ which has successfully been used to fit similar measurements for energies below $300 \mathrm{MeV} .5$ This model describes the DCX process for nuclei with excess neutrons within a single shell in terms of two amplitudes, usually denoted $A$ and $B$. The $A$ amplitude corresponds to transitions through analog states and involves long-range effects without nucleon correlations. The $B$ amplitude arises from short-range correlations and represents transitions through, or to, nonanalog states. While the model was originally based on seniority-zero wave functions, corrections can be applied which correspond to the use of more realistic full shell-model wave functions and to distortion effects. The model provides expressions both for DIAS cross sections and for nonanalog ground-state cross sections.

We have successfully fitted the DIAS cross sections for ${ }^{42,44,48} \mathrm{Ca}$ plus ${ }^{50} \mathrm{Ti}$ and ${ }^{5} \mathrm{Cr}$ with this model, but we have found that it significantly overpredicts the ground-state cross sections for all $T>1$ nuclei. We also found that DIAS 


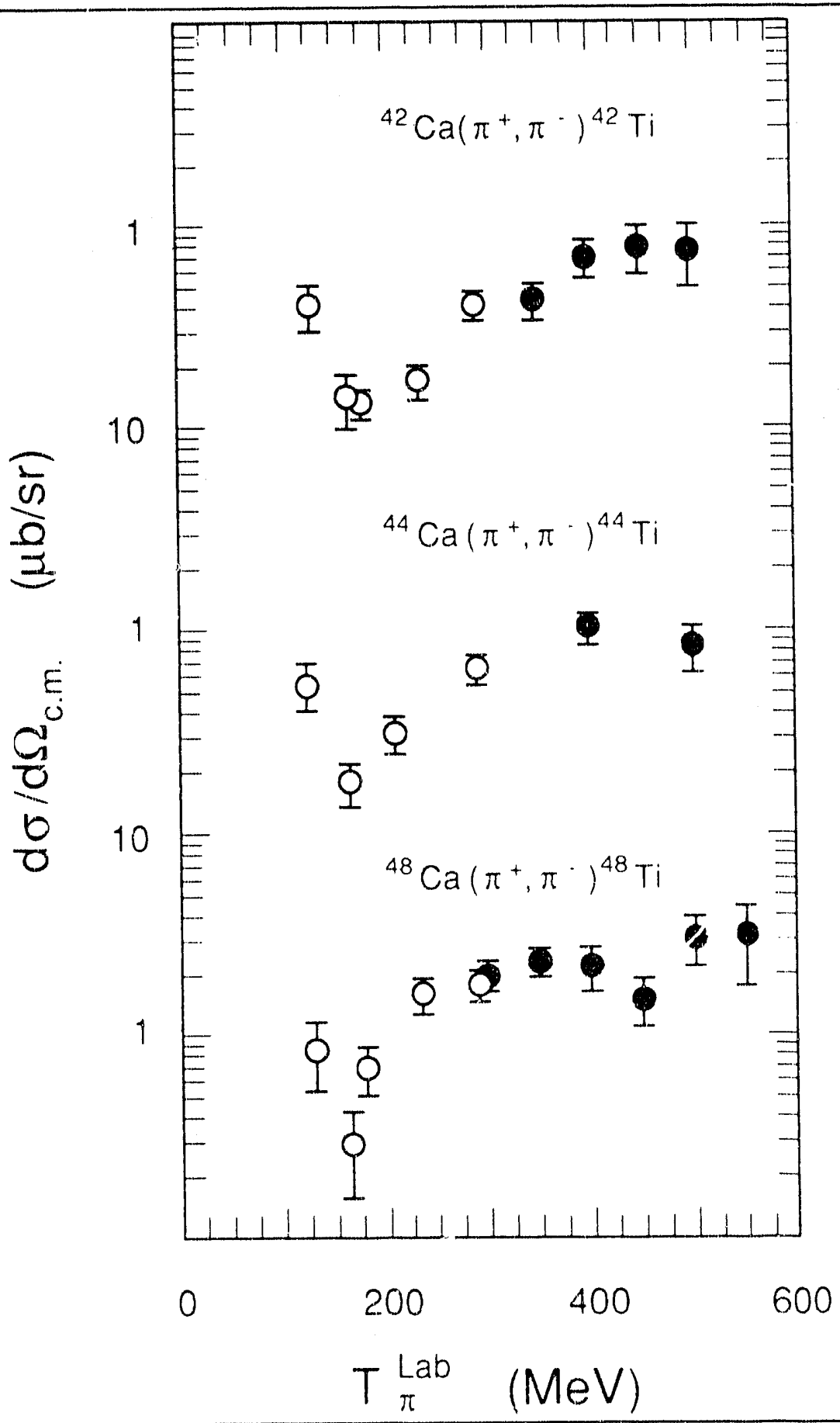

Fig. 1. The $5^{\circ}$ cross sections as a function of encrgy for the DIAS transition in ${ }^{42,44,48} \mathrm{Ca}$. The filled circles are the new mew.drements reported here and the open circles are from previous work. 


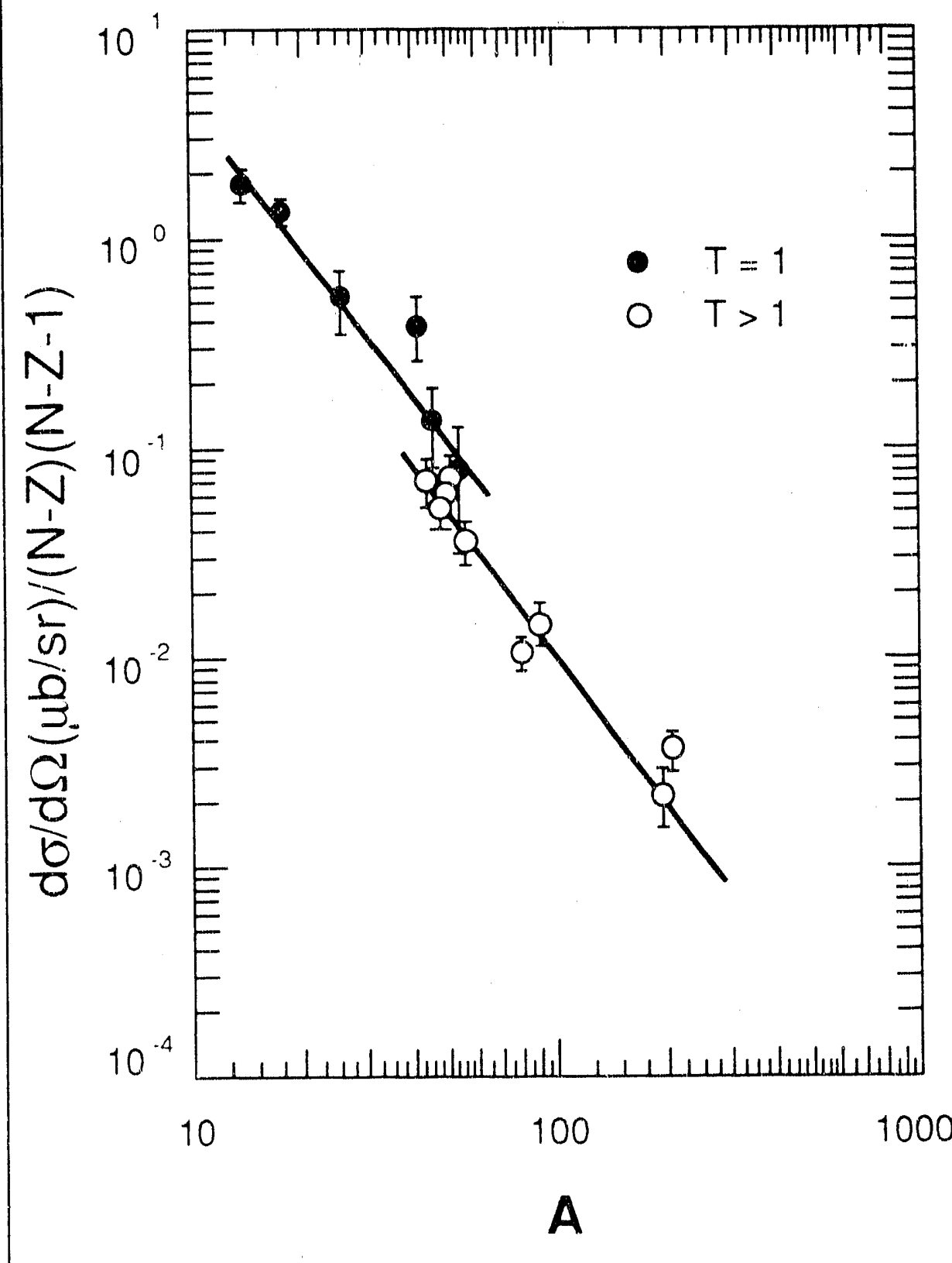

Fig. 2. Small-angle differential cross sections at $500 \mathrm{MeV}$ for the $D C X$ reaction to analog states, divided by $(N-Z)(N-Z-1)$, as a function nuclear mass number $A$. Some $450-\mathrm{MeV}$ data points for nuclei with $A$ between 42 and 54 are also included. The filled circles represent $T=1$ nuclei and the open circles represent $T>1$ nuclei. The solid lines represent an $A^{-T / 3}$ dependence. 
cross sections for the $T=1$ nuclei ${ }^{46} \mathrm{Ti}$ and ${ }^{54} \mathrm{Fe}$ cannot be fitted together with that for ${ }^{42} \mathrm{Ca}$, another $T=1$ nucleus. These results are qualitatively the same, whether or not corrections are applied. Considering the success of this model at lower energies, its failure in this energy range is surprising. Further work, both experimental and theoretical, may give some insight into these discrepancies.

\section{References}

1. A. L. Williams, L. Agnew, L. G. Atencio, H. W. Baer, M. Burlein, G. R. Burleson et al., Phys. Lett. 216B, 11 (1989).

2. R. A. Gilman, Ph.D. thesis, University of Pennsylvania, Los Alamos National Laboratory report LA-10524-T (1985).

3. N. Aucrbach, W. R. Gibbs, and E. Piasetzky, Phys. Rev. Lett. 59, 1076 (1987).

4. N. Auerbach, W. R. Gibbs, J. N. Ginocchio, and W. B. Kaufmann, Phys. Rev. C38, 1277 (1988).

5. M. J. Leitch, presented at the Workshop on Nuclear Structure with Medium Energy Probes, Santa Fe, New Mexico, 1988 [Bull. Am. Phys. Soc. 33, 1548 (1988)]. 
EXPERIMENT $1153-\mathrm{p}^{3}$.
East

\section{Quasifree Pion Charge Exchange at $500 \mathrm{MeV}$}

Univ. of Colorado, MIT,
Argonne

Spokesmon: S. Hoibraiten and R. J. Peterson (Untv. of Colorado)

Participants: M. Braunstein, X. Y. Chen, W. Fong, S. Holbraiten, E. R. XInnoy, M. Kohler, J. J. Kraushoar, B. Kriss, D. Mercer, T. R. Meltvain, D. Oakley, J. Ouyang, R. J. Peterson, D. Prout, R. A. Ristinen, M. Wang, and L. B. Weinstein
During August and September 1989, the $\pi^{0}$ spectrometer was used with $500-\mathrm{MeV} \pi^{-}$and $\pi^{+}$beams at $\mathrm{P}^{3}$-East to measure single-charge-exchange spectra from complex nuclei under kinematic conditions for free pion-nucleon charge exchange. A clear peak corresponding to quasifree scattering is seen after correction for the spectrometer acceptance. A sample inclusive spectrum is shown in Fig. 1, compared to a shape for charge-exchange scattering on a nucleon in a Fermi gas. Effects of pinding energy and finite detector acceptance are not included in that shape.

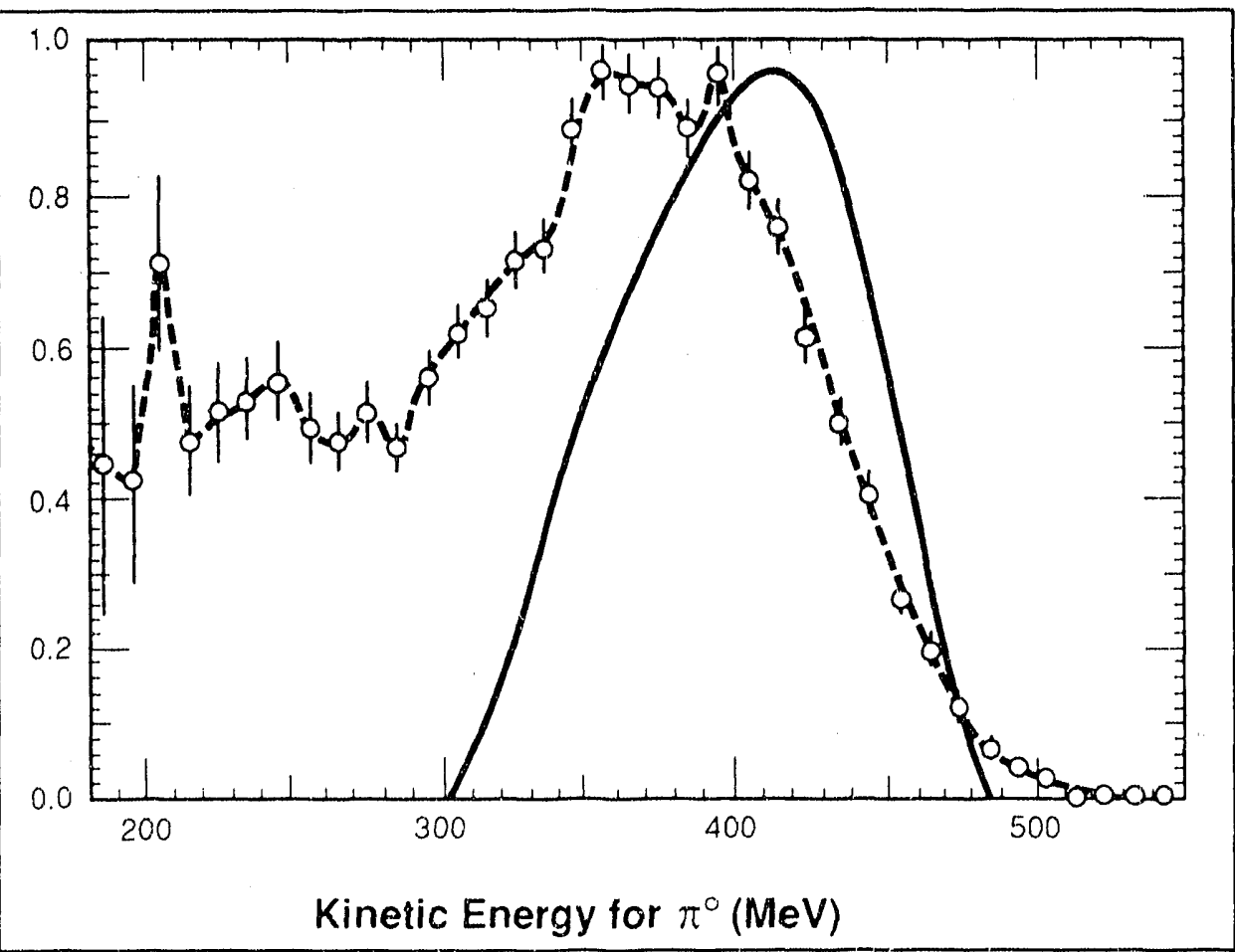

Fig. 1. Negative pion charge exchange on ${ }^{12} \mathrm{C}$ at $500 \mathrm{MeV}$ at an average angle of $50^{\circ}$ (lab) shows a peak representing quasifree scattering on a target proton. The curve shows the peak shape expected for charge exchange on a proton in a Fermi gas, uncorrected for binding energy or the large detector acceptance. A continuum beyond the quasifree peak is also evident. No absolute cross-section scale is given.

Targets from deuterium through bismith were used to vary the ratio of nuclear surface to volume. Angle settings from $0^{\circ}$ to $90^{\circ}$ (lab) were used to vary the momentum transfer. Pauli blocking decreases the inclusive scattering yield below about $30^{\circ}$ (lab). Full angular distributions were taken for seven targets, while five extra targets were examined at $50^{\circ}$ to establish a mass dependence at the maximum of the yield. 
Recoil protons were detected in an array of eight plastic scintillator $\Delta E-E$ telescopes, centered on the direction of the momentum transfer. Coincidence data, with good accuracy, were obtained for five targets with $\pi^{+}$beams at $30^{\circ}$, $50^{\circ}$, and $70^{\circ}$. At $0^{\circ}$ the recoil, momentum was too low to detect protons, while at $90^{\circ}$, the proton energies were too high to stop in the telescopes. The proton telescopes were calibrated with protons of known energy from the channel.

At the middle angles for $\pi^{+}$, a broad pattern of coincident protons was observed, in general reflecting the target Fermi momentum, with no more than slight differences for difterent heavy targets. With $\pi^{-}$, enough recoil protons were observed to indicate second-order reaction processes initiated by recoil quasifree neutrons.

On-line analyses are highly promising, and more detailed work is proceeding smoothly to establish accurate features of quasifree pion charge exchange at an energy high enough to yield a simple reaction picture. 
EXPERIMENT 1015 -- PSR

\section{A Large Cerenkov Detector for Neutrino Physics}

\author{
UC Irvine, UCLA, UC \\ Riv orside, Univ. of \\ Collirado, CEBAF, \\ College of William \& \\ Mary, Los Alamos, Univ. \\ of New Mexico, Univ. of \\ Pennsylvania, Temple \\ Univ.
}

Spokesman: D. H. White (Los Aiamos)

Participants: A. C. Allen, X.-Q. Lu, G. J. lgo, E. Gülmez, $C$. Whitten, G. VanDalen, Sun Yul Fung, J. Kang, J. Layter, B. C. Shen, R. Ristinen, R. Smythe,

R. C. Carlini, R. Burman, J. Donahue, D. Fitzgerald, W. Foreman, M. Hoehn, $T$. Kozlowski, D. M. Lee, W. C. Louis, R. Macek, J. McGill, E. C. Milner, M. Oothouct, V. Sandherg, G. Sanders, M. Schillaci, R. Werbeck, $R$. Westervelt, $D$. H. White, $D$. Whitehouse, M. L. Brooks, B. B. Dieterle, C. P. Leavitt, R. Reeder, W. Frati, A. K. Mann, M. Newcomer, R. van Berg, L. A. Auerbach, V. L. Highland, W. K. McFarlane, and R. T. Seigel
A collaboration from Los Alamos, University of California at Irvine, University of California at Los Angeles, University of California at Riverside, Continuous Electron Beam Accelerator Facility (CEBAF), University of Colorado, University of New Mexico, University of Pennsylvania, Temple University, and College of William and Mary has proposed an experiment to measure neutrino-electron scattering to high accuracy. From a measurement of the ratio

$$
R=\sigma\left(\nu_{\mu} e\right) /\left[\sigma\left(\nu_{e} e\right)+\sigma\left(\bar{\nu}_{\mu} e\right)\right],
$$

$\sin ^{2} \theta_{W}$ will be determined to a precision of $1 \%$; a comparison of this value of $\sin ^{2} \theta_{W}$ to that obtained from the measured masses of the $W$ and $Z$ bosons provides a sensitive test of the Standard Model of electroweak interactions and a promising means to learn about physics beyond the Standard Mcidel. Other physics topics that can be studied in the experiment include searches for neutrino oscillations, limits on the neutrino-charge radius or magnetic moment, leptonnumber violation, supernova-neutrino bursts, and upward-going muons in the $\mathrm{GeV}$ range.

The detector consists of a 7000-ton imaging water Cerenkov tank with approximately 10,000 photomultiplier tubes of 10-in diameter lining the surfaces of the tank. The LAMPF Proton Storage Ring (PSR) will produce a proton pulse of $2.5 \times 10^{13}$ protons in a duration of $270 \mathrm{~ns}$ at a $45-\mathrm{Hz}$ repetition rate, corresponding to an average current of $180 \mu \mathrm{A}$. These protons interact in a copper beam stop that will be located near the center of the detector and will be well shiclded to reduce neutron backgrounds to acceptable levels. Positive pions produced in the beam stop will quickly come to rest and decay in the sequence $\pi^{+} \rightarrow \mu^{+} \nu_{\mu}$, which will be followed by muon decay according to $\mu^{+}-e^{+} \nu_{e} \bar{\nu}_{\mu}$. The experiment will take advantage of the 270-ns PSR beam spill in order to separate the neutrinos from pion decay (26-ns lifetime) from the neutrinos from muon decay (2200-ns lifetime); because the different neutrinos will be produced in almost exactly equal numbers from pion and muon decay at rest, the beam stop source will provide neutrino beams with well defined spectra. Electrons scattered by the neutrinos will produce Cerenkov light, which is then detected by the photomultiplier tubes. From a reconstruction of the hit phototubes, the position, angle, time, and energy of the electron will be determined to good accuracy.

A considerable amount of work has gone into designing and simulating the detector and understanding the backgrounds that can be expected from the experiment. An initial design has been completed, along with enginecring studies and a cost estimate. The worst backgrounds appear to be the beam-associated backgrounds from neutron interactions in the water and neutrino-oxygen scattering, although othe. backgrounds such as cosmic rays, nonuniform efficiency, and systematic time and energy shifts have also been studied. Measurements of neutron-related background have commenced. It has been estimated that for a complete run of 625 days, the systematic error in the determination of $\sin ^{2} \theta_{W}$ will be roughly half of the statistical error, and the estimated total error is approximately $0.9 \%$. 
The first prototypes of the new 10-in photomultiplier tube were shipped to Los Alamos by Burle Industries. Early test results show that these tubes greatly exceed our expectations and that a new very high quality, large, fast photomultiplier tube has been brought into existence. A time resolution of $2.3 \mathrm{~ns}$ FWHM has been observed (Fig. 1) for one photoelectron per event and full-face illumination. Single photoelectrons are resolved (Fig. 2) with a peak to valley ratio of 3:1.

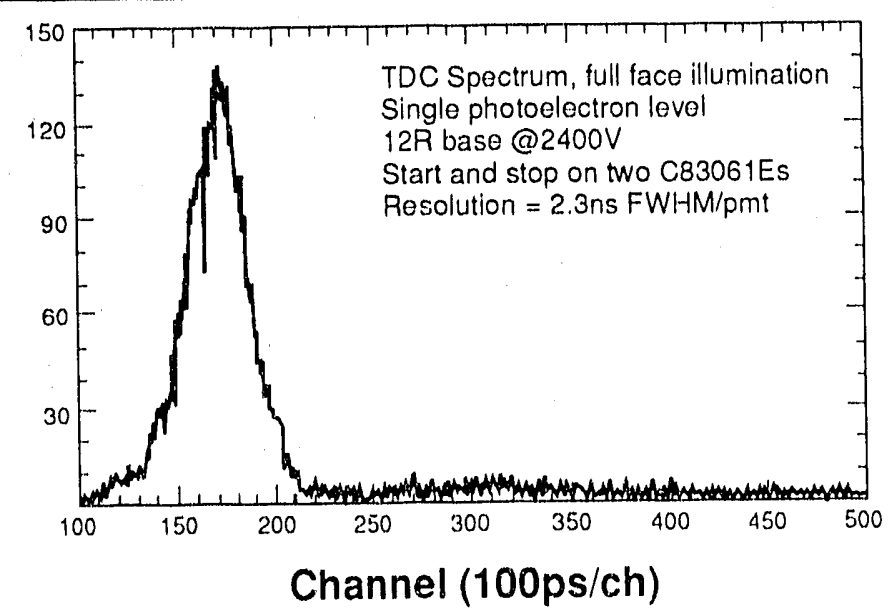

Fig. 1. The TDC spectrum resulting from the full face illumination at the single photoelectron level of two C83061E photomultiplier tubes. One tube triggered the TDC start, and the other tube the TDC stop. The timing resolution corresponds to 2.3 ns FWHM/tube.

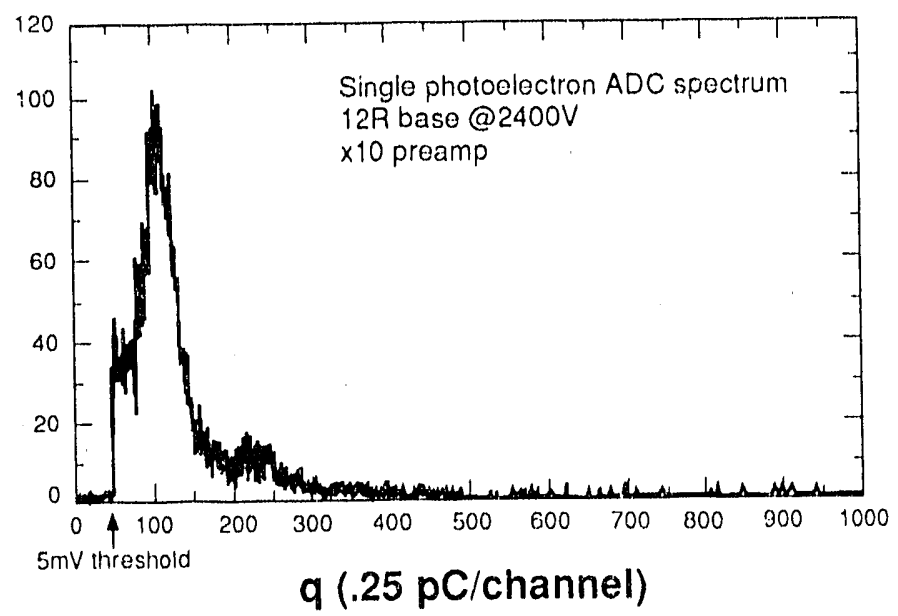

Fig. 2. The $A D C$ spectrum resulting from the full face illumination of a C83061E photomultiplier tube. The dominant peak is due to single photoelectrons and is clearly separated from the broader, two-photoelectron peak. 
The final design of the experiment will be completed in the next year. In addition to cinstinued simulations of the detector, various supplementary measurements $w i l l$ be made to insure that the experimental design is adequate for a precise determination of $\sin ^{2} \theta_{W}$. These measurements include studies of pion and photon production from background neutrons, low-energy neutron fluxes from the Exp. 225 beam dump, and Čerenkov photon detection from an electron beam in water. Furthermore, development work will proceed on each major system of the experiment. Additional photomultiplier tubes will be evaluated; front-end electronics and data-acquisition design will continue; iron for neutron shielding will be procured; and beam-line magnet design will begin. The group is planning to start construction in about one and one-half years and to have first beam to the detector in about three and one-half years.

Much development work is underway. Fifty 10-in. photomultiplier tubes (in addition to the five previously ordered) have been ordered from Burle Industries and should arrive soon. In addition, an initial design of the front-end electronics and data acquisition system has been completed, and prototyping has begun. Finally, an experiment measuring pion production from neutron interactions on various nuclei has finished and will provide important information on background neutrons once the data are fully analyzed. 
EXPERIMENT 1183 - PSR

\section{The Study of Time Reversal and Parity Symmetries Using Epithermal Neutrons}

\author{
Los Alamos, North Carolina \\ State Univ., Triangle \\ Universities Nuclear \\ Laboratory, Duke \\ U'niv., TRIUMF, Univ. \\ of Technology (The \\ Netherlands), Max-Planck- \\ Institut (W. Germany), \\ Univ. of Arizona
Spokesman: J. D. Bowman (Los Alamos)
Participants: J. D. Bowman, J. Knudson, S. Penttilï, S. J. Soestrom, J. J. Szymariski, V. W. Yuan, C. R. Gould, D. G. Hasse, G. E. Mitchell, N. R. Roberson, P. P. J. Delhell, \\ H. Postma, and E. D. Davis
}

The goal of this work is to search for symmetry violations by measuring the dependence of the transmission of epithermal neutrons on quantities such as $s \cdot K, s \cdot J \times K$ and $(s \cdot J \times K)(J \cdot K)$. The quantity $s \cdot K$, neutron helicity, measures parity nonconservation, while $s \cdot J \times K$ and $\left(s \cdot J \times K^{\prime}\right)(J \cdot K)$ test for time-reversal symmetry violation with and without parity violation, respectively. The total cross section for epithermal $(1-10,000 \mathrm{eV})$ neutrons interacting with a nuclear target of mass $A$ exhibits resonances when the cross section is plotted as a function of neutron energy. These resonances correspond to states in the daughter, mass $A+1$, nucleus. Since these resonances have an excitation of 6 to $7 \mathrm{MeV}$ relative to the ground state of the daughter, they occur with a high density-one resonance per $10 \mathrm{eV}$. Because the resonances are close to one another, the effects of a weak symmetry violation nucleon-nucleon force are amplified. A small mixing-matrix element can produce a large symmetry-violating adinixture in the wave function of a nuclear state because the admixture is the ratio of the mixing-matrix element to the spacing between states, which can be small.

The large fluxes of neutrons available at the Los Alamos Neutron Scattering Center (LANSCE) allow high statistical accuracies. The time structure of the LAMPF beam is compressed in the Proton Storage Ring so that a 250-ns wide pulse of protons impinge on a tungsten spallation target. The resulting spallation neutrons per proton are moderated in water to obtain an epithermal neutron spectrum. The neutrons are polarized $(40 \%)$ by selective absorption in a cell of polarized hydrogen. The spin of the neutron beam is reversed every few seconds by a system of magnetic fields. The neutrons have their spins either parallel or antiparallel to their momentum $(s \cdot K=+$ or - ) as they interact with the nuclear target.

Not every nuclear state is expected to exhibit parity nonconservation. For a spin-zero target, states that have neutron orbital angular momentum 1, $p$-wave, and total spin $1 / 2$ are good candidates. Such states can mix with states of opposite parity that have orbital angular momentum $0, s$-wave, and total spin $1 / 2$, and exhibit large parity nonconserving, helicity dependent, cross sections. In the summer of 1989, we searched for parity nonconservation in $17 p$-wave resonances at neutron kinetic energies of between 10 and $250 \mathrm{eV}$. We observed larger-than-two standard deviation effects in three resonances. This is the first time that more than one instance of parity nonconservation has been observed in a single target and the first time at energies above a few eV.

The nuclear states at this excitation are extremely complex-so complex that their properties are best treated by considering the states to be chaotic. If the states are expanded in a shell model basis, then thousands of states will be involved in the representation of each nuclear state. The expansion coefficients, as well as matrix elements between states, are random variables. The paritynonconserving matrix elements between nuclear states are expected to have a Gaussian distribution centered about zero. From our data, we have determined $m$, the width of the Caussian distribution. $m$ is the root mean squared mixing matrix element, which is found to be $0.58-0.050+0.25 \mathrm{meV}$. The likelihood 
function of $m$ is non-Gaussian. A plot of the likelihood function of $m$ vs $m$ is shown in Fig. 1. The nonzero results set the lower limit for $m$ and the results consistent with zero set the upper limit. This is the first experimental determination of $m$.

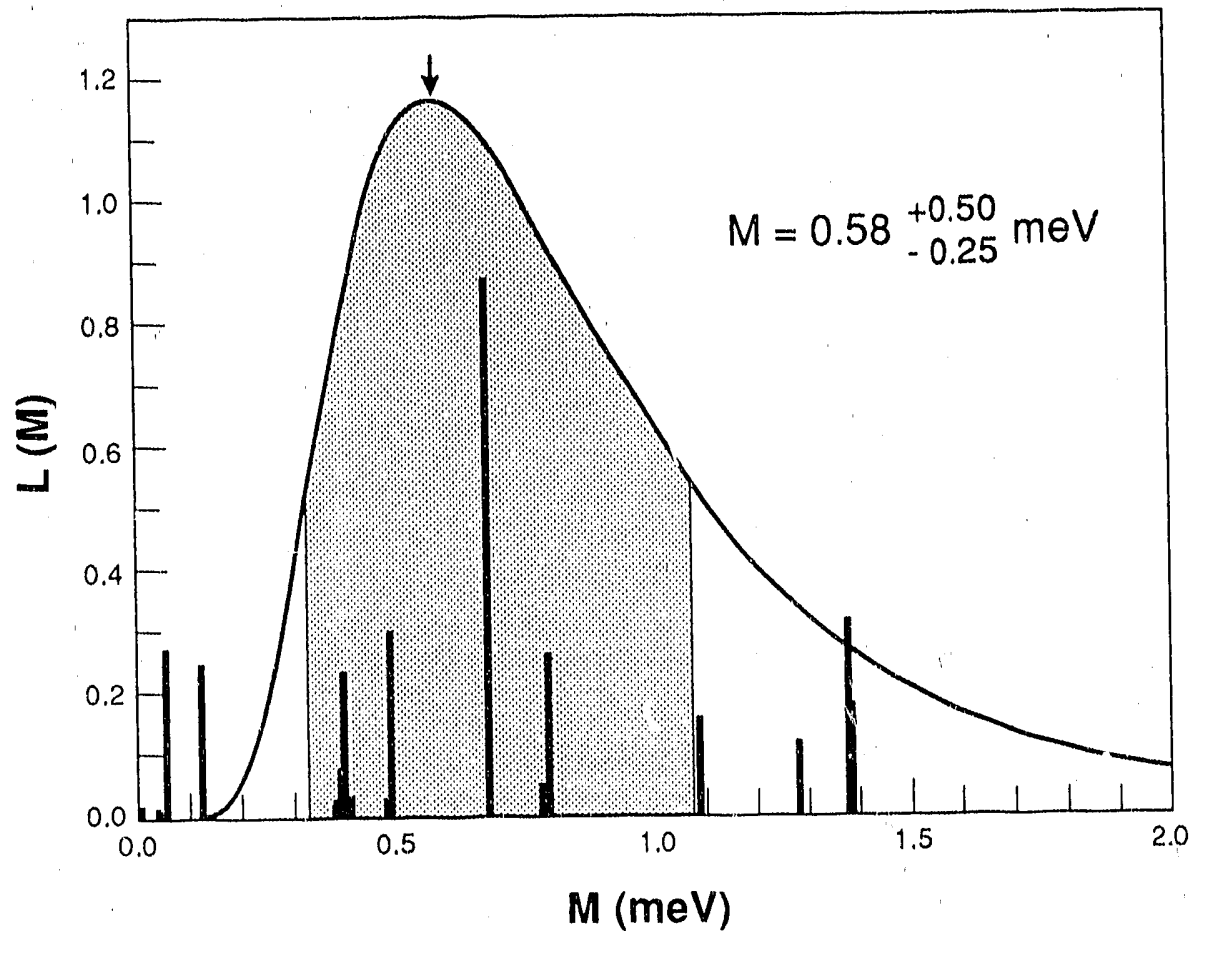

Fig. 1. Likelihood function of the root-mean-squared mixing matrix element $M$. The location of the vertical bars is the absolute value of the $Q^{\prime}$ s (see text) and the heights are proportional to the statistical signijicance. The shaded region is the $68 \%$ confidence interval. 
EXPERIMENT 969 - SMC

\section{MEGA: Search for the Rare Decay $\mu^{+} \rightarrow e^{+} \gamma$}

UCLA, Univ. of Shicago, Univ. of Houston, Fermilab, Los Alamos, Princeton Univ., Stantord Univ., Texas A\&M Univ., Valparaiso Univ., Univ. of Virginia, VPI, Univ. of Wyoming, Yale Univ.

\section{Spokesman: M. D. Cooper (Los Alamos)}

Participants: D. Barlok, R. Kessler, B. M. K. Nefkens, J. Price, J. Crocker, S. C. Wright, P. S. Cooper, M. Dzemidzic, J. Flick, E. V. Hungerford III, $K$. Johnston, K. Lan, B. W. Mayes II, L. Pinsky, R. Pheips, L. Tang, W. von Witch, J. F. Amann, R. D. Bolton, M. D. Cooper, W. Foreman, R. Harrison, G. Hant, C. M. Hoffman, N. Hotfman, G. E. Hogan, N. June, D. Kercher, J. Little, T. Kozlowskl, R. E. Mischke, F. J. Naivar, J. Novak, M. A. Oothoudt, $C$. PIllal, S, Schilling, W. Smith, S. Stanis/aus, J. Sturrock, J. Szymanskl, R. D. Werbock, D. Whitehouse, C. Wilkinson, A. Hallin, E. B. Hughes, C. Jul, J. N. Otis, C. Gagliardi, G. Kim, F. Lk, R. E. Tribble, L. Van Ausdeln, R. J. Fisk, D. D. Kootke, R. Manweller, R. Marshall, W. Stephens, B. Tippens, K. O. H. Zlock, L. E. Piilonen, A. R. Kunselman, and J. K. Markey
The search for $\mu \rightarrow e \gamma$ at LAMPF is called MEGA, an acronym standing for Muon decays into an Electron and a GAmma ray, and is an experiment with a branching ratio sensitivity of roughly $10^{-13}$. Such a search is important because there is no reason for conservation of muon family number, though the observation of the decay' wo:ld indicate the need for an extension to the Standard Model of electroweak interactions. During the summer of 1989, the experiment had one run, the purpose of which was to test detector performance. This run culminated the year's work and its results are presented here.

The MEGA apparatus is shown in Fig. 1. The apparatus is contained in a large solenoidal magnet with a $1.5 \mathrm{~T}$ field, a clear bore of $1.85 \mathrm{~m}$, and a length of $2.9 \mathrm{~m}$. The cryogenics system for the magnet is largely monitored by a computer that automates refilling of cryogens and gives telephone alarms for abnormal conditions; this system consiáerably reduces the manpower requirements for maintenance.

\section{(a)}

(b)
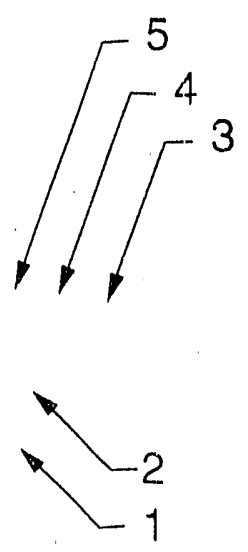

(c)

(d)

Fig. 1. A cutaway view of the MEGA apparatus.

The required muon stopping rate is $3 \times 10^{7} \mathrm{~Hz}$ (average). Development runs at the Stopped Muon Chan ${ }^{-} \mathrm{el}$ (SMC) have demonstrated an excess of flux from the channel. Tunes have been developed that discard the excess in a way that very little beam is dropped near the apparatus. There is no evidence that minor changes in the tune induce backgrounds in the photon spectrometer. The 
beam separator operates reliably for multiple week periods at voltages in excess of $200 \mathrm{kV}$ and is used to remove the positron contamination from the beam.

The detector is divided into a positron spectrometer and a series of photon pair spectrometers. All of the charged particles arising from muon decay are confined by the magnetic field to a maximum radius of $29 \mathrm{~cm}$, leaving the photon detectors in a relatively quiet environment.

The positron arm consists of two parts, a set of multiwire proportional chambers (MWPC) for momentum determination and scintillators for timing. The MWPC design for the positron spectrometer is called "Snow White and the Seven Dwarfs." There is a central, cylindrical chamber of $11.75-\mathrm{cm}$ radius whose cathodes are kept relatively clean by instrumenting only their ends. Seven small "dwarf" chambers encircle "Snow White" to provide the necessary redundancy for pattern recognition.

Two dwarf chambers have been assembled and tested in the beam. A chamber construct consists of two cathodes and an anode supported on cylindrical end pieces. The cathodes are $25-\mu \mathrm{m}$ Kapton foils with an evaporated layer of $\mathrm{Cu}(1 / 4$ $\mathrm{ohm} /$ square) for the electrode. The foils are striped using a computer controlled scribe to produce stripes $3-\mathrm{mm}$ wide for electronic readout of induced signals. The precise cylindrical shape, good to $\pm 75 \mu \mathrm{m}$, is maintained by a 20-torr differential gas pressure across the foils. The differential pressure is maintained by an electronically controlled gas system capable of regulating many gas volumes simultaneously and adjusting the chamber pressure between local Los Alamos pressure and sea level pressure. The anodes are $15-\mu \mathrm{m}$ gold-coated tungsten wire, strung with a pitch of $1 \mathrm{~mm}$. The end pieces are made of precisely machined ceramic that hold the half-gap of the chambers at $1.75 \mathrm{~mm}$.

The chambers are $1.26 \mathrm{~m}$ in length. In cider to prevent electromechanical instabilities, the wires must be supported at four places along their length with "garlands." The garlands consist of a $25-\mu$ m nylon monofilament that is in dividually glued to the wires to maintain spacing, a $0.6-\mathrm{mm}$ glass fiber to add rigidity, and six thin-walled posts to support the structure off the inner foil.

The chambers are constructed on a central rod that supports the end pieces and the tension of the wires. Before installation in the beam, the chambers are mounted in a cylindrical shell whose wall lies outside the $29-\mathrm{cm}$ radius of decay positrons. The tension of the wires is transferred to the shell and the construction rod is removed to leave a nearly massless detector of $3 \times 10^{-4}$ radiation lengths. The installation of a chamber into the shell takes a half day of work. The low mass minimizes the photon background from electromagnetic interactions of the decay positrons.

The chambers are operated at 760 torr with a gas mixture of $80 \%$-CF4:20\%isobutane. Anode plateau voltage is at 2700 volts; the cathode plateau is about 100 volts higher. The chambers are capable of taking the full bearn intensity, but their efficiency under those conditions is not known yet.

The predicted performance of the MECA experiment has been based on Monte Carlo simulations and design studies. The verification of the agreement 
between detector performance and design studies is an important step toward data taking. The data are still in analysis, but several qualitative features appear to be checked:

1. The frequency pattern of wire firings agrees with the simulation.

2. The spiraling of the positrons through the chambers confirms that the multiple scattering is properly taken into account.

3. The widths of hit clusters from single chamber crossings roughly agree with those predicted.

All indications are that the chambers should operate as designed.

The second part of the positron spectromcter consists of banks of scintillators arranged in a cylindrical geometry about the beam near the pole faces. The positrons enter the scintillators after at least one revolution in the chambers. Positrons trapped in the field for more than eight loops are rejected by their relative time with respect to the photon. Each bank is divided into 90 elements to keep the individual counter rates manageable. Two sections of 43 scintillators, one upstream and one downstream of the target, were installed this year; they covered the same solid angle as the dwarfs.

The scintillator dimensions are $0.0 \times 0.9 \times 30 \mathrm{~cm}^{3}$ (rhomboid cross section), and they are attached to $1.5-\mathrm{m}$ fiber optics light guides, necessary to get the photomultiplier tubes outside the magnetic field. The resolution for detecting decay positrons is roughly 0.6 ns Full Width at Half Maximum (FWHM), a value slightly greater than the experimental design.

The photon arm is four concentric pair spectrometers of essentially identical construction. One pair spectrometer was available for evaluation in the beam run. Each pair spectrometer is made of lead converters, MWPCs, drift chambers, and scintillators. The detailed arrangement is shown in Fig. 2. The scintillators continue to perform according to specifications.

The MWPC never reached a sufficient voltage to have high efficiency because of mechanical difficulties in the construction. Reinforcing the converter cylinders with woven carbon fibers is a major design change that is intended to solve the mechanical problems. The laminate of lead, carbon fiber, and Rohacell will be constructed by a commercial firm that guarantees the required tolerances.

The drift chambers came onto plateau. Recognizable pair events have allowed a comparison between Monte Carlo simulated showers and actual events. Two types of photon data were taken. The first is from photons produced from the reaction $\pi^{-},-\pi^{n} n$, which have an energy spectrum that is flat from 55 to $82 \mathrm{MeV}$. These events appear to be extremely similar to the simulated events. The second type of data was from the radiative decay of muons, which have an energy spectrum peaked at zero energy. These events are less spread in azimuth in the detector than the signal being sought. A couple of samples of each type of data are shown in Fig. 3, along with some hand drawn reconstructions. The 


\section{PHOTON ARM EVENT}

52.8 MEV $\gamma$

$\begin{array}{cc}\text { Aluminum Scint. } & \text { Rohacell } \\ \text { Lead }\end{array}$

Fig. 2. An exploded view of a pair spectrometer layer seen parallel to the magnetic field.

body of data is still in analysis. The similarity of the data to the simulation is encouraging evidence that the pair spectrometers will operate as designed.

A long-standing problem has been the determination of the longitudinal components of the photon position and momentum. The design requirement is for $5 \cdot \mathrm{mm}$ (FWHM) resolution for the position of chamber crossings. Two solutions have been found and tested in prototype chambers. The one to be implemented is a staggered delay line that runs along the cathode of the innermost drift chamber of each layer. There will be one delay line for each drift chamber cell. Induced signals on the cathode will propagate along the dulay line at about $0.3 \mathrm{~cm} / \mathrm{ns}$. From a measurement of the difference of arrival times at the two ends of the chamber, the longitudinal position can be measured to the desired accuracy.

The MECA trigger relies on an electronics circuit that measures the azimuthal width of hit patterns from photon-induced pairs. One such module has been built and partially tested. The module uses programmable array logic and has a propagation delay of less than $30 \mathrm{~ns}$. No problems with the circuit have been found, althougt a complete test will have to await a working photon MWIC. 


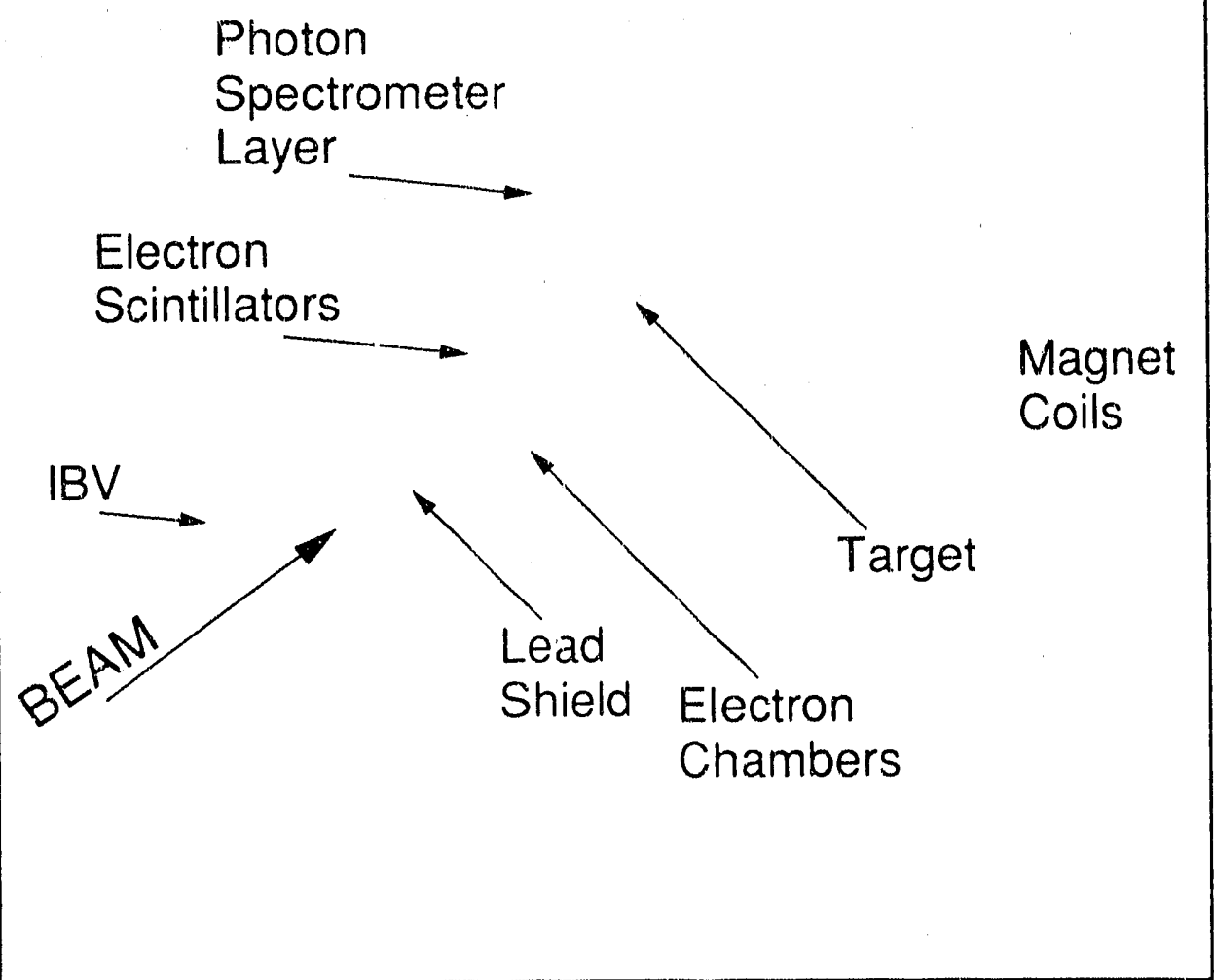

Fig. 3. Sample high-energy photon eveni in the pair spectrometer. Pictures (a) and (b) are from the $\pi^{-} p \rightarrow \pi^{0} n$ and $\pi^{0} \rightarrow \gamma \gamma$ process; pictures (c) and $(d)$ are from the $\mu \rightarrow$ eq $\nu \bar{\nu}$ decay. The elements in the pictures are: (1) scintillators, (2) converter, (3) drift chariver 1, (4) drift chamber 2, and (5) drift chamber 3. The schematic reconstructions guide the eye as to the development of the event.

The expected trigger rates can be inferred from data in the scintillators and drift chambers. Figure 4 plots the two types of trigger rates as a function of the percentage of the design intensity for. AEGA. The highest rates come from a condition placed only on the photon scintillators. A substantial accidental component is identified at the highest rates. Once the drift chambers are required, the accidentals are suppressed as are the coincidences from very low energy processes. The agreement with Monte Carlo predictions is quite good. 


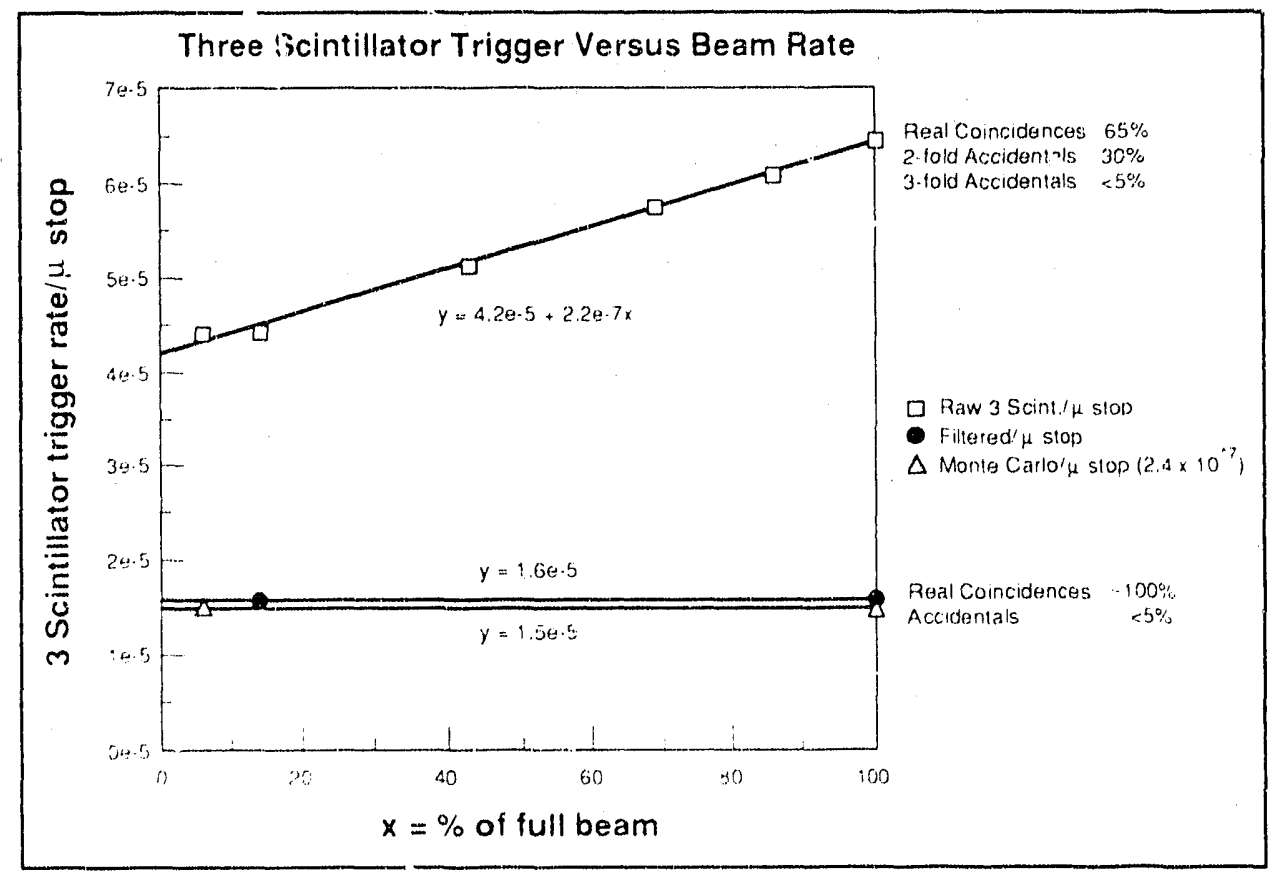

Fig. 4. Trigger rates for various trigger conditions relevant to MEGA as a function of stopping intensity. The Monte Carlo simulation is shown for the drift chamber included conditions. Also shown are the decompositions into single and multiple (accidental) photon events.

The MECA data acquisition system consists of FASTBUS front ends and memories, a VME-based microprocessor farm, and a MicroVAX control computer. The new item this year was the arrival of production quantities of the FASTBUS modules. Both wire-chamber latches and time-to-digital converters (TDC) existed in quantity. The pulse height modules are expected in the spring of 1990. Expansion of our system to three full crates of FASTBUS did not introduce any substantive problem. The MEGABLOCK memory transfer, where all module memories are treated as a single memory, worked well and saved many microseconds of data readout time.

A calibration task is to monitor the time drifts of all the photomultiplier tubes over the years of data taking. Coincidences between a timing counter and each of the scintillators in the experiment are accumulated. The data are collected in histograms in the Advanced Computer Project (ACP) microprocessors. With the speed of parallel processing, all the required time spectra for the counters can be accumulated in just a few minutes.

In the next year, the collaboration hopes to bring together enough of the detector to begin data taking in 1990. Nothing was discovered in 1989 that prevents the experiment from achieving its goals. Sufficient equipment to set new limits on the $\mu-$ er process may be available in 1990, with the more stringent measurements in 1991. 


\section{Astrophysics}

\section{CYGNUS Project}

Los Alamos, Univ. of Maryland, George Mason Univ., UC Irvine, Univ. of Notre Dame, Univ. of Leeds (UK)

Spokesmen: C. M. Hotfman and D. E. Nagle (Los Alamos)

Participants: R. L. Burman, C. M. Hoffiman, D. E. Nagle, M. Potter, V. D. Sandberg, S. Stanislaus, C. A. Wilkinson, W. Zhang, D. Berley, C. Y. Chang, B. L. Dingus, J. A. Goodman, T. J. Haines, M. Stark, D. E. Alexandreas, R. C. Allen, S. Biller, R. S. de Laye, G. M. Dion, X-Q. LU, P. R. Vishwanath, G. Yodh, R. W. Ellsworth, D. R. Cady, and J. Lloyd-Evans
The CYGNUS detector at LAMPF is an extremely powerful extensive air shower array that detects Ultra High Energy (UHE, $100 \mathrm{TeV}-10 \mathrm{PeV}$ ) cosmic gamma rays. The detector looks for discrete sources of UHE gamma rays (such as neutron stars and black holes) to understand the mechanism of the cosmic accelerators within these sources and to study the interactions of the UHE gamma rays themselves. The CYGNUS detector corsists of 107 scintillation counters, each with an area of $0.85 \mathrm{~m}^{2}$, deployed around the LAMPF beam stop and covering an area of $\sim 2 \times 10^{4} \mathrm{~m}^{2}$; the MWPC's from the neutrino detector, formerly used in LAMPF Exp. 225, are used as tracking muon detectors. The experiment started operation in 1986, albeit with a smaller number of detectors at the beginning, and has been in continuous operation since. The university collaborators are funded by the National Science Foundation (NSF), while the Los Alamos component has been funded by Laboratory ISRD funds.

Observations over the past five years have established that the compact $x$ ray sources, Cyg $X-3$ and Her $X-1$, are also sources of UHE gamma rays. The observations, the most compelling of which was published by the Cygnus Collaboration in Physical Review Letters, also show that the gamma-ray interactions in the atmosphere produce far more muons than expected. Either the nature of photon interactions changes dramatically at these extremely high energies, or the primary particle is some hitherto unknown light, neutral particle. Either of these possibilities indicates exciting new physics. In addition, the measured period for UHE gamma rays from Her X-1 is significantly different from the measured $x$-ray period.

The array is presently undergoing expansion to 213 detectors, covering an area of $5 \times 10^{4} \mathrm{~m}^{2}$, and thereby greatly increasing the sensitivity of the array. Some of the scintillation counters from Exp. 225 are being installed underground to provide additional muon detection. The new detectors and their electronics have been deployed and are undergoing tests; the array should be complete and taking data by the end of 1989.

An extensive data set, spanning the years 1986-1989, is undergoing intensive analysis. A series of papers has been submitted to XXI International Cosmic Ray Conference describing the results of this analysis.

\section{Publications and Papers}

T. J. Haines, D. E. Alexandreas, R. C. Allen, S. Biller, D. Berley, R. L. Burman et al., "A Limit on Possible Energy-Dependent Velocities for Massless Particles," Phys. Rev. D 41, 692 (1990).

D. Berley, C. Y. Chang, B. L. Dingus, J. A. Goodman, T. J. Haines, M. Stark et al., "Observations from the CYGNUS Experiment of Cyg X-3 During the 1989 Radio Burst," in Proceedings of the XXI International Cosmic Ray Conference, Adelaide, Australia, January 6 19, 1990, R. J. Protheroe, Ed. (University of Adelaide, Physics Publications, 1989), Code OG 4.1-6, p. 17. 
D. Berley, C. Y. Chang, B. L. Dingus, J. A. Goodman, T. J. Haines, M. Stark et al., "Search for Signals from X-Ray Binaries from the CYGNUS Experiment at Los Alamos," in Proceedings of the XXI International Cosmic Ray Conference, Adelaide, Australia, January 6-19, 1990, R. J. Protheroe, Ed. (University of Adelaide, Physics Publications, 1989), Code OG 4.6-9, Vol. 2, p. 333.

D. Berley, C. Y. Chang, B. L. Dingus, J. A. Goodman, T. J. Haines, M. Stark et al., "Search for Emission of UHE Gamma Rays from the Crab Nebula," in Proceedings of the XXI International Cosmic Ray Conference, Adelaide, Australia, January 6-19, 1990, R. J. Protheroe, Ed. (University of Adelaide, Physics Publications, 1989), Code OG 4.3-9, Vol. 2, p. 154.

D. Berley, C. Y. Chang, B. L. Dingus, J. A. Goodman, T. J. Haines, M. Stark et al., "Search for Diffuse Galactic Emission, Anisotropies, and 'Unknown' Sources in Muon-Rich and -Poor Showers with the CYGNUS Experiment," in Proceedings of the XXI International Cosmic Rai' Conference, Adelaide, Australia, January 6-19, 1990, R. J. Protheroe, Ed. (University of Adelaide, Physics Publications, 1989), Code OG 4.7-2, Vol. 2, p. 387.

D. E. Alexandreas, R. C. Allen, S. Biller, G. Dion, X-Q. Lu, P. R. Vishwanath et al., "Relative Muon Content of UHE Showers Associated with Her X-1," in Proceedings of the XXI International Cosmic Ray Conference, Adelaide, Australia, January 6-19, 1990, R. J. Protheroe, Ed. (University of Adelaide, Physics Publications, 1989), Code OG 4.2-8, Vol. 2, p. 116.

D. Berley, C. Y. Chang, B. L. Dingus, J. A. Goodman, T. J. Haines, M. Stark et al,, "Status of the Expansion of the CYGNUS Array at Los Alamos," in Proceedings of the XXI International Cosmic Ray Conference, Adelaide, Australia, January 6-19, 1990, R. J. Protheroe, Ed. (University of Adelaide, Physics Publications, 1989), Code HE 7.3-12, Vol. 10, p. 301.

R. L. Burman, C. M. Hoffman, D. E. Nagle, M. E. Potter, V. D. Sandberg, D. Berley et al., "A Low Threshold EAS Array for Gamma-Ray Astronomy at Los Alamos," in Proceedings of the XXI International Cosmic Ray Conference, Adelaide, Australia, January 6-19, 1990, R. J. Protheroe, Ed. (University of Adelaide, Physics Publications, 1989), Code HE 7.3-11, Vol. 10, p. 297. 


\section{Atomic and Molecular Physics}

\section{EXPERIMENT 963 - SMC \\ First Direct Measurement of $\alpha-\mu$ Sticking in $d t-\mu \mathrm{CF}^{1}$}

Los Alamos, Brigham Young Univ., Idaho Research Sottware, Idaho National Engineering Laboratory

\section{Spokesman: M. A. Paciottl (Los Alamos) \\ Participants: M. A. Paciottl, O.K. Baker, J. N. Bradbury, J. S. Cohen, $M$ Leon, H. R. Maltind, O. M. Rivera, L. L. Sturgess, S. E. Jones, F. LI, L. M. Roes, E. V. Sheely, J. K. Shurtletf, S. F. Taylor, A. N. Anderson, A. J. Caffrey, and J. M. Zabriskie}

\section{Introduction}

There has been a need for some time for a direct measurement of the $\alpha$ $\mu$ sticking probability in muon-catalyzed $d-t$ fusion. The muon loss caused by this sticking phenomenon is the most severe limitation on the ultimate fusion yield. In this experiment, both $(\alpha \mu)^{+}$and $\alpha$ particles have been observed in coincidence with fusion neutrons in a gascous D-T mixture at approximately $10^{-3}$ liquid-hydrogen density. ${ }^{1}$ This measurement comes very close determining the initial sticking probability, $\omega_{s}^{o}$, in $d t-\mu \mathrm{CF}$. Previously, the effective sticking probability, $\omega_{s}$, has been inferred from the total muon loss rate after detailed corrections ${ }^{2,3,4}$ in much higher density mixtures.

Extension of this work is underway at Rutherford-Appleton Laboratory, ${ }^{5}$ and the direct ionization chamber method, so successful at the Leningrad Nuclear Physics Institute (LNPI) for $d d-\mu$ CF sticking, ${ }^{6}$ is now being used at Paul Scherrer Institute (PSI) with somewhat higher density than Exp. 963.

\section{Concept of the Experiment}

The $(\alpha \mu)^{+}$ions produced by "sticking" events and the $\alpha$-particles formed in the remaining $99+\%$ majority of events are detected in coincidence with the 14.1-MeV neutron and are easily separable by range in the low pressure D-T gas whose density is dictated by the limited ranges of these ions. The fact that the ions are produced at $108^{\circ}$ from the neutrons is useful for background rejection. Triggers caused by $\left(\mu^{-}, m\right)$ and $\left(\mu^{-}, p 2 n\right)$ captures in the target flask are substantially suppressed because the charged particle is not similarly correlated in angle with the neutron. Beryllium was chosen as the flask material because of its low muon-capture probability.

\section{Layout}

An important design feature is protection of the silicon detector from tritium beta radiation. $T_{2}$ diffuses out through the target window $W 1$ (Fig. 1), limited primarily by the aluminum coating, and is diluted by the large volume of the secondary container. The second window W2 then keeps this dilute mixture at a distance from the detector where the intervening $D_{2}$ region is guarded by a magnetic field. Backgrounds are measured in an identical apparatus filled entirely with $D_{2}$ and normalized to incoming muons.

\section{Systematic Effects}

Since the a range is only one-fourth that of the $(\alpha \mu)^{+}$(both $\left.3.5 \mathrm{MeV}\right)$, the target density was reduced by a factor of four for the detection of the $\alpha$ particles. The two densities used are $\phi=7.6 \times 10^{-1}$ (490 Torr) for $\alpha$ and $\phi=$ $2.8 \times 10^{-3}$ (1800 Torr) for $(11 /)^{+}$. Both ions are therefore collected from the full 


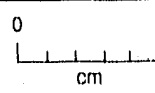
5

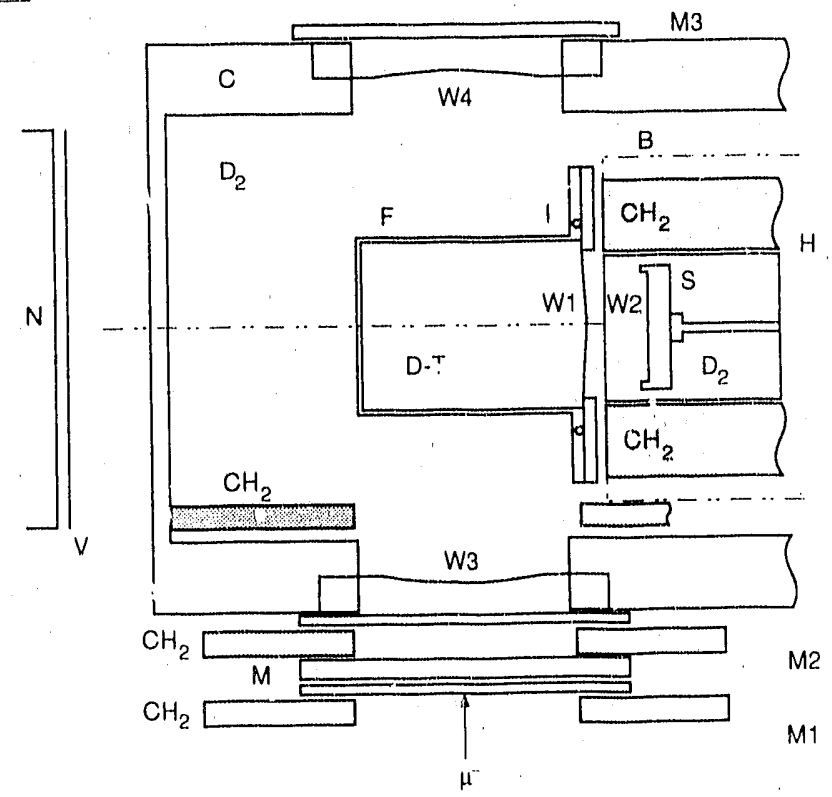

Fig. 1. F: Beryllium task flask, 6.4-cm diameter, $7.6-\mathrm{cm}$ long; cylindrical walls are 1.5 -mm thick; contains $D-T, C_{t}=0.4$

W1,W2: 1.5 micron mylar windows aluminized $1600 \AA$ on $T_{2}$ side

I: Pure indium ' $O$ '-ring sealing the target window

S: Silicon surface barrier detector, $1000 \mathrm{~mm}^{2}, 100$ microns thick

M1,M2,M3: Muon telescope counters, each 1.6-mm thick

$\mathrm{N}$ : Neutron counter, Bicron liquid scintillator (BC-501), $12.7 \mathrm{~cm}$ in diameter and $12.7-\mathrm{cm}$ in depth $1.6-\mathrm{mm}$ veto counter in front

$B$ : Dotted line indicates pole tip of 1-kG permanent magnetic field

C: Secoridary contai zer for $D_{2,}, 12-1$ ' volume, Al walls, Lucite lid, all seals Viton ' $O$ '-rings

W3,W4: Heat-treated Al beam entrance and exit windows, 0.13-mm thick

$H$ : Detector housing, sealed, cooled, and movable; contains $D_{2}$

M: Moderator for slowing down 60-MeV/c $\mu^{-}$beam

$V$ : Charged particle veto counter

target volume, eliminating geometric differences. The normalization of the two fills assumes that the number of fusions per incident muon scales as $\phi$ squared, but several systematic effects could alter this assumption:

1. The muon stopping rate would scale with $\phi$.

2. Any change in gas purity between the two fills would introduce a systematic effect.

3. At the low density, $1 /$ and $/ \mu$ atoms might diffuse to the walls and reduce the yicld. 
4. Change in tritium concentration, $C_{1}$, because of diffusion out the window, could alter the fusion yield.

5. Most importantly, the fusion yicld per stopped muon $\gamma$ might not scale with density.

A Monte Carlo code is useful for evaluating systematic effects. It incorporates a measured muon stopping distribution, detector thresholds $(0.7 \mathrm{MeV}$ for charged particles), and particle range. The overall efficiency is small $(0.08 \%$ per stopped ${ }^{-}$), is believed to be independent of density, and cancels out of the sticking result. The muon stopping rate within the target volume was measured by counting the "Mn activation of thin iron foils placed inside the target. The stop rate in hydrogen, based on these foils, was $4.7 \times 10^{-4} \mathrm{H}^{-}$stopped per incident $\mu^{-}$at 490 Torr.

Stripping of the $11^{-}$reduces the number of observed events, and corrections are accurately handled by the Monte Carlo, using the energy-dependent stripping cross sections for D-'T gas, aluminum, mylar, and $D_{22}$ gas. ${ }^{8}$ Table I lists stripping for the several materials assuming an average energy at each position. The relative effects of the gas and of the windows are approximately equal.

Table I. Stripping Probabilities.

\begin{tabular}{|c|c|}
\hline Stripping Material & $\begin{array}{c}\text { Stripping } \\
\text { Probability } \\
(\%)\end{array}$ \\
\hline $3.9-\mathrm{cm}$ average $\mathrm{D}-\mathrm{T}$ path length at 1800 Torr & 6.5 \\
\hline $1600-\AA \mathrm{Al}$ coating on target window & 1.3 \\
\hline 1.5-micron mylar targei window & 4.0 \\
\hline $1.0-\mathrm{cm} \mathrm{D}_{2}$ at $1800 \mathrm{Torr}$ & 1.0 \\
\hline $160(0)-\AA$ Al coating on detector window & 1.1 \\
\hline 1.5-micron mylar detector window & 3.9 \\
\hline $1.5-\mathrm{cm} \mathrm{D}$, at 1800 Torm & 2.1 \\
\hline
\end{tabular}

Many of the stripped ( $r / 1)^{+\prime}$ s still have enough energy to be detected above threshold as a particles, and as a consequence, only $16 \%$ of the initially produced $(r y /)^{t ' s}$ are unobserved. Stripping is significantly less than that seen when the ion is allowed to stop fully in a medium " and makes our method uniquely suited to obtaining $\omega_{s}^{0}$.

\section{Data}

The r-data are presented first because the signal is so prominent (Fig. 2). The time difference between the $r$-ion and the fusion neutron is plotted along the abscissa while the ion energy is plotted along the ordinate. The box drawn 


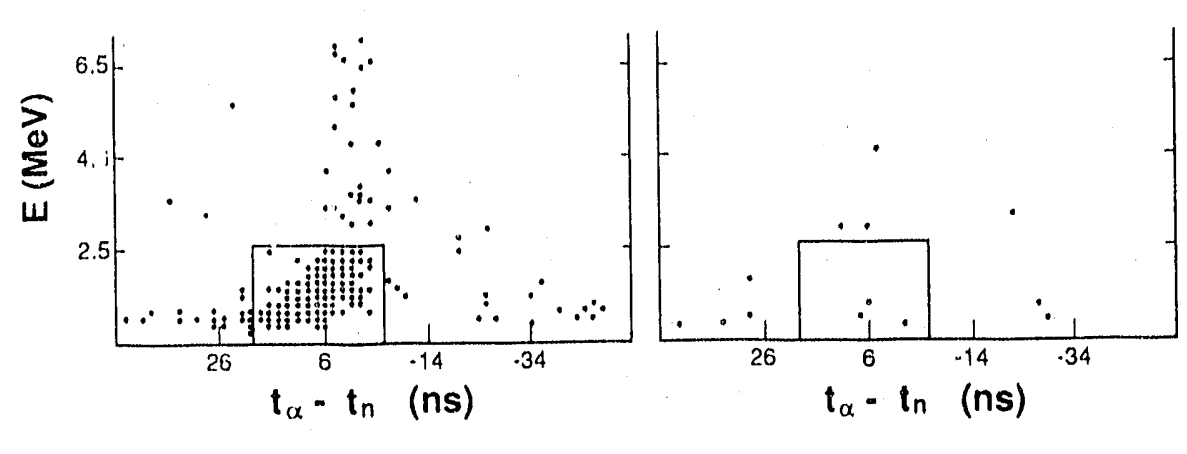

Fig. 2. $\alpha$-data on the left. Background on the right for $1 / 7$ number of incident muons.

shows the region where the $a^{\prime}$ s are expected from Monte Carlo predictions. The position of the box along the time axis cannot be known from measurer ent, so the $x$-data themselves are used as a guide to positioning the box. $(\alpha \mu)^{+}$ions are also expected in this plot, but with such low rate that background masks them.

The $(\alpha \mu)^{+}$spectrum for all data taken at 1800 Torr is shown in Fig. 3, where the axes are the same as in Fig. 2. A quick comparison with its companion background plot shows a strong signal, but of course not as clean as the $\alpha$ data. The coincident background, which extends from threshold to well above the maximum $(1 / 1)^{+}$energy, comes from from $\mu^{-}$capture in the beryllium
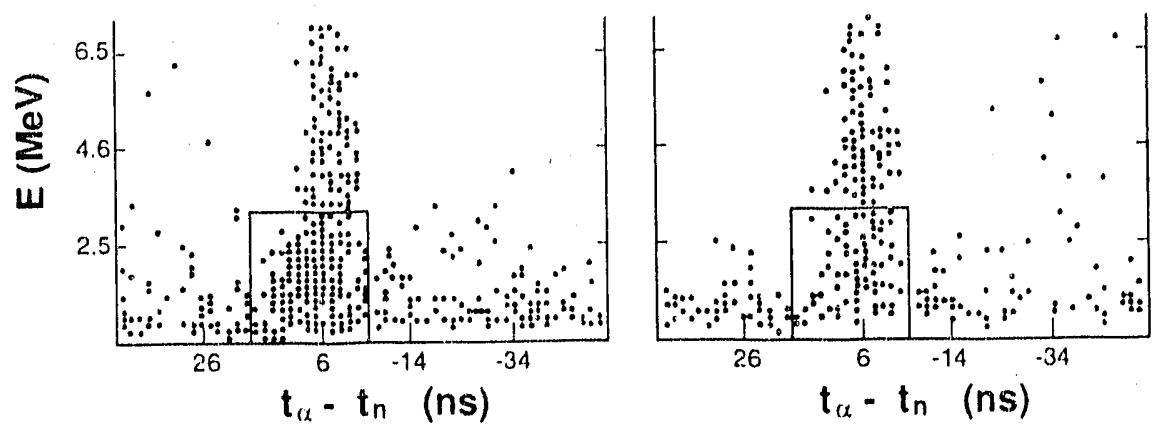

Fig. 3. The $(\alpha / \mu)^{+}$spectrum for all data taken at 1800 Torr. Background is shown on the right for $3 / 4$ number of incide. : muons. 
target flask; protons, deuterons, tritons, and alphas are emitted in coincidence with neutrons. Noncoincident background, in the wings of the time distribution, originates from a varicty of sources producing singles, including scattered muons which cannot be completely rejected and products from $\mu^{-}$capture in beryllium, polyethylene, and aluminum.

The initial sticking probability is simply

$$
\omega_{s}^{0}=\frac{N_{\alpha, \mu}}{N_{\alpha}+N_{\alpha \mu}}
$$

where $N_{\alpha}\left(N_{\alpha \mu \mu}\right)$ is the number of doubly (singly) charged ions detected, and $N_{\alpha \mu}$ has been adjusted for the $16 \%$ that were stripped. The counts are normalized to the number of incident muons at each fill pressure. Assuming that both the number of $\mu^{-}$stopped and the yield $x$ are proportional to $\phi$, then it follows that $\omega_{s}^{o}=(0.80 \pm 0.14) \%$ (statistical crror).

\section{Systematic Uncertainty}

An anticipated source of trouble was impurities in the D-T mixture, since the mylar window precludes a high-temperature bake-out of the target. An effective procedure used was to fill first to 1800 Torr and to collect $(\alpha \mu)^{+}$data, then to bleed the gas pressure down to 490 Torr and collect $\alpha$ data. In this way, the same gas sample was used for the measurement and for the normalization.

The $t \mu$ and $d \mu$ diffusion distances are limited by the muon lifetime at such low pressures and could become somewhat longer because of diffusion while the atoms are still epithermal. We estimate this distance to be about $1 \mathrm{~cm}$ at 490 Torr, somewhat smaller than the dimensions of the target, so no correction is made at this time.

At our densities, $1 /$ thermalization times equal or exceed the muon lifetime. Therefore, most of the molecular formation will occur in the epithermal (transient) region where $\lambda_{\| 1 /}$ is rapidly changing, and the yield $\chi$ per stopped muon may not scale with density. Table II outlines the scope of this problem. Thermalization times, as well as average temperature and remaining triplet fraction at a relevant time of $2 \mu \mathrm{s}$ (all from Ref. 10), tell us that at the lowest density the $t \mu$ atoms remain hot, and the triplet quenching is not complete. The muon lifetime then selects rather different slices of the epithermal transient for each density.

Estimating singlet and triplet formation rates for our mixture at the two average temperatures, we find a higher rate for the less thermalized, low-density case. ${ }^{1 ?, 13}$

The other pertinent quantities are $\eta_{1}$, (the probability of $d \mu$ reaching the ground state) and $\lambda_{11}$ (the 1,11 triplet quenching rate). An extrapolation of the Menshikov and Ponomarev $/ 1 s$ is presented in Table II for each density; " the direction of the $/ 1$, density dependence is to offset the increased formation rate expected at the low density. In this simplified treatment, the 0.33 triplet fraction 


\begin{tabular}{lcc}
\hline \hline Table II. Density Effect. & & \\
\hline Quantity & $\mathbf{4 9 0}$ Torr & $\mathbf{1 8 0 0}$ Torr \\
\hline Density $\phi$ & $7.6 \times 10^{-4}$ & $2.8 \times 10^{-3}$ \\
Thermalization time & $6 \mu \mathrm{s}$ & $1.6 \mu \mathrm{s}$ \\
Average temperature at $2 \mu \mathrm{s}$ & $540 \mathrm{~K}$ & $340 \mathrm{~K}$ \\
$t \mu$ triplet fraction at $2 \mu \mathrm{s}$ & 0.33 & 0.04 \\
Average $\lambda_{\text {dt } \mu}$ & $1.5 \times 10^{8}$ & $1.3 \times 10^{8}$ \\
Extrapolated $q_{1 *}$ from Ref. 11 & 0.77 & 0.66 \\
\hline \hline
\end{tabular}

remaining in the 490-Torr sample has little effect on the conclusion; in the event that $\lambda_{10}$ is actually larger than calculated, ${ }^{14}$ the triplet fraction could approach zero without largely affecting the $\lambda_{d \ell \mu}$ listed.

Based on the reliability of the above assumptions, we judge it unlikely that the density effect will alter $\omega_{s}^{o}$ by more than $15 \%$. Accordingly, a systematic uncertainty is quoted with the result: $\omega_{s}^{o}=(0.80 \pm 0.15 \pm 0.12$ systematic $) \%$. Subsequent experiments or calculations on the density effect can be used to correct this result.

The initial $\left(C_{t}^{\prime}\right.$ of 0.4 falls with time, and ionization chamber measurements indicate $C_{1}$ dropped to 0.38 during the 1800 -Torr fill and to 0.37 during the 490 Torr fill. This small change could be significant only if the optimum $C_{t}$ is much higher than expected because of epithermal molecular formation.

\section{Conclusion}

We report a measurement of the initial $\alpha-\mu$ sticking probability in muon catalyzed $\left(1-1\right.$ fusion at low density. $\omega_{s}^{0}$, measured directly for the first time, is $(0.80 \pm 0.15 \pm 0.12$ systematic)\%, in agreement with "standard" theoretical calculations ${ }^{15,16,17}$ and not supporting those theories that invoke special mechanisms to alter the initial sticking. ${ }^{18,19}$ The reported value contains only a $16 \%$ correction to the observed sticking because of stripping. The Exp. 963 equipment has been moved to the pulsed muon beam at the Rutherford-Appleton Laboratory where a high- $Z$ target, substituted for beryllium, produces lower backgrounds.

\section{Acknowledgements}

We wish to thank R. H. Sherman of the Los Alamos National Laboratory for making a test of the cleanliness in tritium of the thin mylar coated with aluminum.

This work is supported by the U.S. Department of Energy Division of Advanced Energy Projects. 


\section{References}

1. M. A. Paciotti, O. K. Baker, J. N. Bradbury, J. B. Cohen, M. Leon, H. R. Maltrud et al., in Proceedings of the „CF Workshop, Sanibel Island, Florlda, 1988, S. E. Joncs, J. Rafelski, and H. J. Monkhorst, Eds. [AIP Conf. Proc. 181, $38(1988)]$.

2. S. E. Jones, A. N. Anderson, A. J. Caffrey, C. De W. Van Siclen, K. D. Watts, J. N. Bradbury et al., Phys, Rev. Lett. 56, 588, (1986).

3. C. Petitjoan, W. H. Brcunlich, M. Cargnelli, P. Kammel, J. Marton, N. Nägele et al., Muon Catal. Fusion 1, 89 (1987).

4. C. Petitjean, P. Ackerbaucr, W. H. Breunlich, M. Cargnelli, M. Jeitler, P. Kammel et al., Muun Catal. Fusion 2, 37 (1988).

5. J. Davies, in Proceedings of the 1 CF Workshop, Sanibel Island, Florida, 1988, S. E. Jones, J. Rafelski, and H. J. Monkhorst, Eds. [AIP Conf. Proc. 181, 52 (1988)].

6. A. A. Vorobyov, Muon Catal. Fusion 2, 17 (1988).

7. G. Heusser and T. Kirsten, Nucl. Phys. A 195, 369 (1972).

8. J. S. Cohen, Phys. Rev. A 37, 2343 (1988).

9. J. S. Cohen, Phys. Rev. Lett. 58, 1407 (1987).

10. J. S. Cohen, Phys. Rev. A 34, 2719 (1986).

11. L. I. Menshikov and L. I. Ponomarev, Pis'ma Zh. Eksp. Teor. Fiz. 39, 542 (1984) [Sov. Phys. JETP Lill. 39, 663 (1984)].

12. M. Leon, Phys. Rew. Lett. 52, 605 (1984), and corrected figures in 52, 1655 (1984). (Caution is advised because of the emergence of the subthreshold resonances as strong contributors to the molecular formation.)

13. J. S. Cohen and M. Leon, Phys. Rev. Lett. 55, 52 (1985).

14. M. Leon, in Procendings of the ${ }^{\prime} C F$ Workshop, Sanibel Island, Florida, 1988, S. E. Jones, J. Rafelski, and H. J. Monkhorst, Eds. [AIP Conf. Proc. 181, 94 (1988)].

15. L. N. Bogdanova, L. Bracci, G. Fiorentini, S. S. Gerstein, V. E. Markushin, V. S. Melezhik ot al., Nucl. Phys. A 454, 653 (1986).

16. D. Ceperley and B. J. Alder, Phys. Rev. A 31, 1999 (1985).

17. Chi-Yu Hu, Phys. Rev. A 34, 2536 (1986).

18. J. Rafelski and B. Müller, Phys. Lett. 164B, 223 (1985).

19. M. Danos, B. Müller, and J. Rafelski, Muon Catal. Fusion 3, 443 (1988). 
EXPERIMENT 1151 - SMC

\section{Experimental Investigation of Muon-Catalyzed Fusion}

\section{Idaho Research, UCLA, Idaho National Engineering Laboratory, Los Alamos, Brigham Young Univ., Univ. of Mississippi \\ Spokesmen: S. E. Jones (Brigham Young Univ.), M. Leon, and M. A. Paciottl (Los Alamos) \\ Participants: A. N. Anderson, K. A. Aniol, J. D. Baker, J. N. Bradbury, A. J. Caftrey, J. S. Cohen, S. E. Jones, M. Leon, H. R. Maltrud, M. A. Paclottl, J. J. Reidy, O. M. Rivera, E. O. Sheely, S. F. Taylor, H. "Vootverton, and J. M. Zabriskie}

A new target system has been assembled for the LAMPF muon-catalyzed fusion experiments, which detect the $14-\mathrm{MeV}$ fusion neutrons that are released following the negative muon-induced $d l$ fusion reaction. This system allows $D_{2}$ and $T_{2}$ to be transferred to the target separately at low temperature 7 ; under these conditions the build-up of the DT fraction is expected to be rather slow. In fact, during the first experiment with this system in September 1988, no change in the experimental cycling rate at $23 \mathrm{~K}$ (liquid target), $\lambda_{c}(23 \mathrm{~K})$ (for tritium fraction $c_{t}=0.7$ ), was observed over a period of sixteen hours, while the temperature was kept alternately at $23 \mathrm{~K}$ and $10 \mathrm{~K}$ (solid target). From this we tentatively draw two conclusions:

1. The build-up of DT is negligibly slow for $T \leq 23 \mathrm{~K}$; and

2. Para- to ortho- $D_{2}$ transitions, which are expected to proceed at an observable rate at $10 \mathrm{~K},{ }^{1}$ either do not occur or else do not affect $\lambda_{c}(23 \mathrm{~K})$ significantly.

Another important comparison was for $\lambda_{c}(23 \mathrm{~K})$ following (1) cooling in the copper-lined target from room temperature over a couple of hours, and (2) extremely rapid cooling resulting from transfer of the gas from a room temperature stainless steel tark into the target at $T \leq 25 \mathrm{~K}$. Since each procedure gave the same $\lambda_{r}(23 \mathrm{~K})$, we draw a third conclusion: cooling in the target from room temperature leaves the $d-t$ mixture with the high temperature equilibrium (h.t.e.) ratio of molecular species $\left(c_{\mathrm{D}_{2}}: c_{\mathrm{DT}}: c_{\mathrm{T}_{2}}=c_{d}^{2}: 2 c_{d} c_{t}: c_{t}^{2}\right)$.

At low temperature $(T<200 \mathrm{~K})$, the resonance model of molecular formation ${ }^{2}$ implies that only total spin zero $t \mu^{\prime}$ 's colliding with $D_{2}$ molecules contribute significantly to molecular formation. ${ }^{3}$ Furthermore, for high tritium fraction $\left(c_{t} \gtrsim\right.$ $0.7)$ the expression for the cycling rate [Ref. 3, Eq. (3)] simplifies to

$$
\lambda_{c} \simeq \lambda_{(d t \mu) d}^{0}\left(\mathrm{C}_{2}\right.
$$

This expression can be readily tested using the new target system.

Experimental 23-K cyclirig rates measured in August and September 1989 are shown as functions of $c_{t}$ in Fig. 1 based on preliminary data analysis; the higher points are for $D_{2}+T_{2}$ targets, the 'ower for h.t.e. targets. For $c_{1} \geq 0.7$, the values of $\lambda_{c}(23 \mathrm{~K}) / c_{\mathrm{D}_{2}}$ are shown in Fig. 2. According to Eq. (1), these points should fall on a single horizontal line. Clearly, they do not. This is shown in another way by forming the ratios

$$
R\left(c_{t}\right) \equiv \frac{\lambda_{c}\left(23 \mathrm{~K}_{1} \mathrm{D}_{2}+\mathrm{T}_{2}\right)}{\lambda_{c}(23 \mathrm{~K}, \text { h.t.e. })}
$$

Eq. (1) implies $R\left(c_{t}\right)=c_{d}^{-1}$, while the experimental ratios fall significantly short of this value (see Table I). 


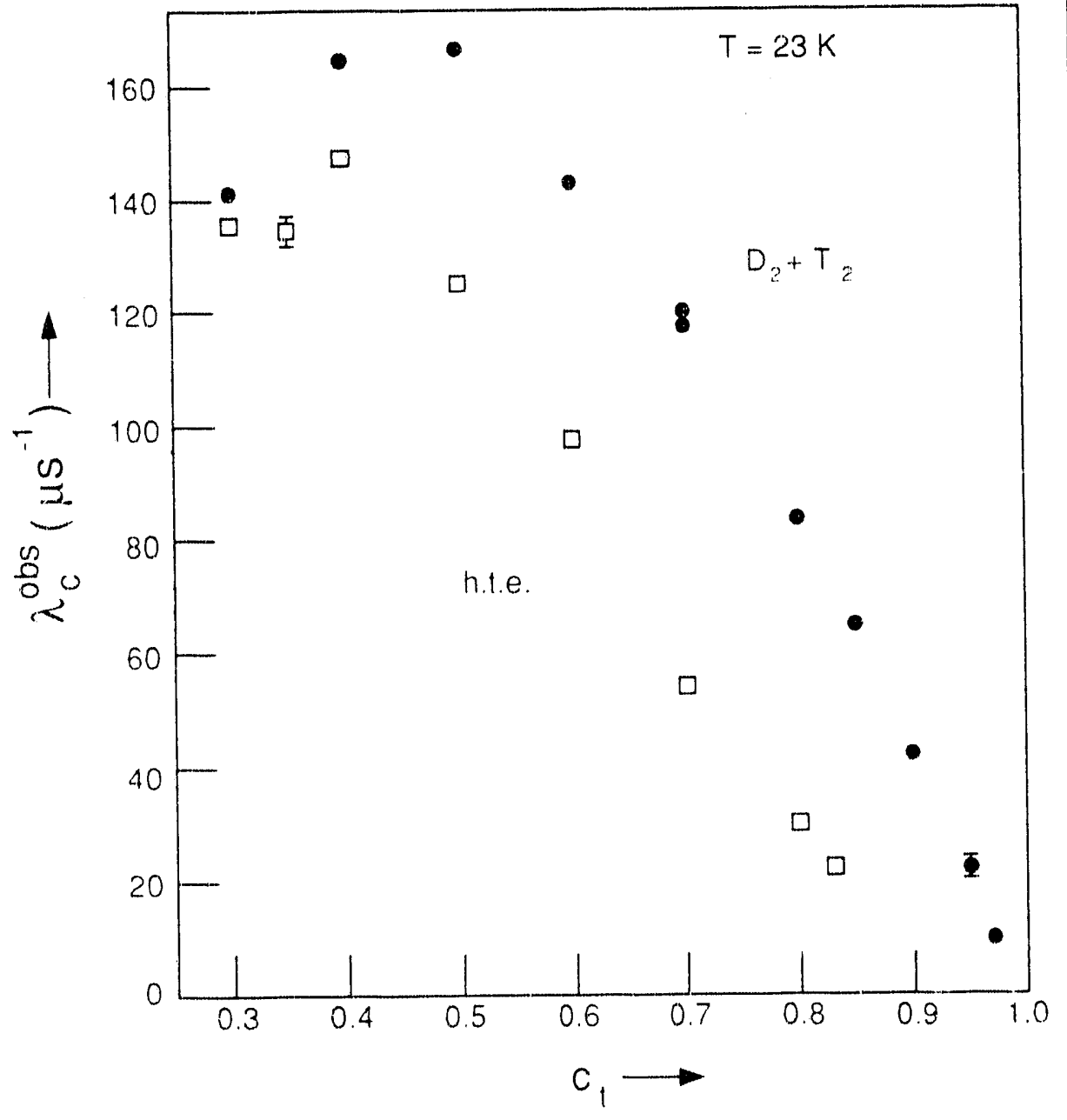

Fig. 1. Observed (not normalized) cycling rates for $T=23 \mathrm{~K}$ from the recent LAMPF experiment (preliminary values, statistical errors only). The upper set of points is for $D_{2}+T_{2}$ targets, the lower set for high-temperature equilibrium (h.t.e.) targets. 


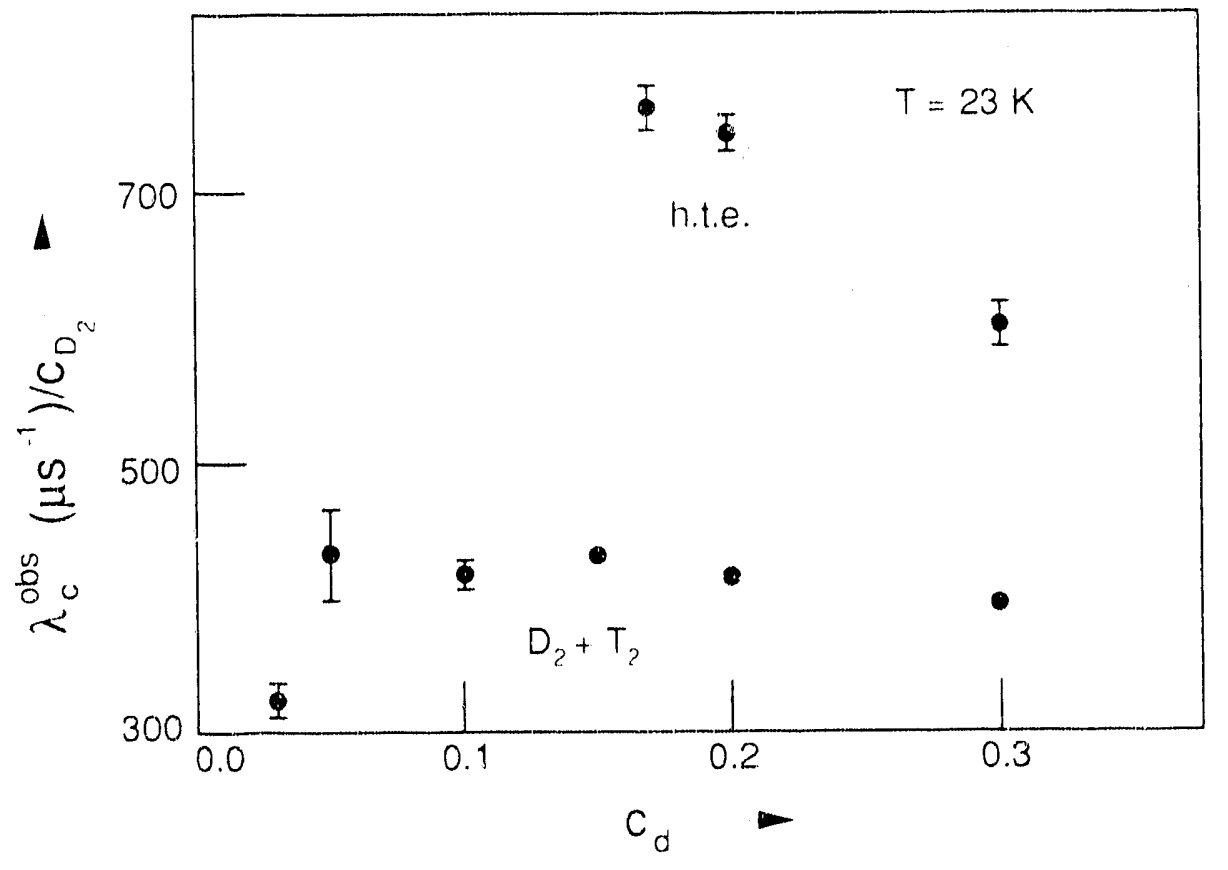

Fig. 2. $\lambda^{o l, s} / c_{\mathrm{D}_{2}}$ for $c_{t} \geq 0.7$, as in Fig. 1 .

Table 1. Experimental Cycling Rate Ratios, $\left(D_{2}+T_{2}\right) /$ h.t.e.

\begin{tabular}{lccc}
\hline$c_{8}(\%)$ & 70 & 80 & 83 \\
$c_{d}^{-1}$ & 3.33 & 5.00 & 5.88 \\
$R(\exp )^{*}$ & $2.17 \pm 0.07$ & $2.80 \pm 0.09$ & $3.27 \pm 0.09$ \\
\hline
\end{tabular}

- Preliminary values, statistical crrors only.

These results were very surprising for us. What do we make of them? We consider the three conclusions discussed above to be solidly based (of course they should be tested further and we plan to do so) and the expectation that only singlet-t $\mu$ on $D_{2}$ is resonant for thermalized $1 \mu$ 's to be even more so); consequently, we are led, as the least iconoclastic resolution, to the tentative conclusion that there are significant epithermal contributions to the steady-state molecular formation rates at $23 \mathrm{~K}$. A similar conclusion might be drawn from the results of the global fits to carlier LAMPF cycling rate data carricd out by Anderson, ${ }^{4}$ which found a $\lambda_{(d t \mu) t}^{0}$ that did not vanish at low $T$. 
Important tests of this explanation of the anomalous $R^{\prime} s$ will come from the observation of the transients in neutron production implied by the large contribution of epithermal molecular formation.

\section{References}

1. P. C. Souers, Hydrogen Properties for Fusion Energy (University of California Press, Berkeley, 1986) p. 317.

2. E. A. Vesman, Pis'ma Zh. Eksp. Teor. Fiz. 5, 113 (1967) [JETP Lett. 5, 91 (1967)].

3. M. Leon, Phys. Rev. Lett. 52, 605 (1984).

4. A. N. Anderson, in Proceedings of the $\mu$ CF Workshop, Sanibel Island, Florida, 1988, S. E. Jones, J. Rafelski, and H. J. Monkhorst, Eds. [AIP Conf. Proc. 181, $57(1988)$ ]. 


\section{Materials Science}

EXPERIMENT 1115

\section{Magnetic Ordering in $\left(\mathrm{Y}_{1-x} \mathrm{Pr}_{x}\right) \mathrm{Ba}_{2} \mathrm{Cu}_{3} \mathrm{O}_{7}$ as Evidenced by Muon Spin Relaxation}

\section{Los Alamos, Memphis State Univ., Texas Tech Univ., San Jose State Univ., Temple Univ.}

\section{Spokesman: D. W. Cooke (Los Alamos) \\ Participants: D. W. Cooke, R. S. Kwok, M. S. Jahan, R. L. Lichii, T. R. Adams, C. Boekema, W K. Dawson, A. Kebede, J. Schwegler, J. E. Crow, and T. Mihalisin}

\section{Introduction}

Using the zero-field muon-spin-relaxation ( $\mu \mathrm{SR})$ technique we have found clear evidence for antiferromagnetic ordering of $\mathrm{Cu}$ moments within the $\mathrm{CuO}$ planes of $\left(\mathrm{Y}_{1-x} \mathrm{Pr}_{x}\right) \mathrm{Ba}_{2} \mathrm{Cu}_{3} \mathrm{O}_{7}$. The Néel temperatures are approximately $285 \mathrm{~K}$, $220 \mathrm{~K}, 35 \mathrm{~K}, 30 \mathrm{~K}$, and $20 \mathrm{~K}$ for $x=1,0.8,0.6,0.58$, and 0.54 , respectively. For $x=$ 0.50 we observe a fast-relaxing component of the muon polarization in addition to a long-time tail, reminiscent of spin-glass behavior. This region of the phase diagram $(0.5 \leq x \leq 0.54)$ corresponds to the existence of both superconductivity and magnetism. The fully developed local magnetic ficld for $x>0.54$ is found to be $\sim 16 \mathrm{mT}$, but decreases to $\sim 12 \mathrm{mT}$ at $T=17 \mathrm{~K}$ for the $x=1$ sample, presumably because of the onset of Pr-ion ordering. Magnetic ordering also occurs in $\mathrm{PrBa}_{2} \mathrm{Cu}_{3} \mathrm{O}_{6}$; the Néel temperature is $\sim 325 \mathrm{~K}$.

The interest in $\left(\mathrm{Y}_{1-x} \mathrm{Pr}_{x}\right) \mathrm{Ba}_{2} \mathrm{Cu}_{3} \mathrm{O}_{x}$ stems from several factors. First, unlike the other rare-earth-based superconductors, $\mathrm{PrBa}_{2} \mathrm{Cu}_{3} \mathrm{O}_{7}$ ( $\mathrm{PBCO}$ ) is insulating rather than metallic or superconducting. It is not understood why the substitution of Pr quenches superconductivity when other rare-earth element substitutions produce superconductors with $T_{\mathrm{c}} \sim 90 \mathrm{~K}$. Experimentally it has been found that $T_{c}$ vs $x$ follows the classic Abrikosov-Gor'kov pair-breaking curve with $T_{c} \rightarrow$ $0 \mathrm{~K}$ for $x \cong 0.6$, leading to the suggestion that the depairing mechanism is presumably caused by strong $\mathrm{f}$-spd hybridization between the nearly tetravalent $\mathrm{Pr}$ ions and the charge carriers in the adjacent $\mathrm{Cu}-\mathrm{O}$ planes. ${ }^{1}$

Secondly, the system remains orthorhombic for $0 \leq x \leq 1$; therefore, complete pseudo-binary alloys of $\mathrm{YBa}_{2} \mathrm{Cu}_{3} \mathrm{O}_{7}(\mathrm{YBCO})$ and $\mathrm{PBCO}$ can be prepared. In addition, the oxygen content remains stable for $0 \leq x \leq 1$. Because this system exhibits both superconductivity $(x \leq 0.6)$ and magnetism (Pr-moment ordering for $0.4 \leq x \leq 1.0$ ), and because it remains orhthorhombic for $0 \leq x \leq 1$, it is a unique system for investigating the interplay between these two ground states without complications which arise from structural changes or variable oxygen content.

We have used zero-field muon-spin relaxation ( $\mu \mathrm{SR}$ ) to examine the magnetism of $\left(\mathrm{Y}_{1-x} \mathrm{Pr}_{x}\right) \mathrm{Ba}_{2} \mathrm{Cu}_{3} \mathrm{O}_{7}$ as a function of concertration and temperature. In addition to the previously reported superconductivity and magnetism (Pr ordering), we find that antiferromagnetic ordering also occurs and that the associated Néel temperatures are concentration dependent. We attribute this magnetism to ordering of $\mathrm{Cu}$ moments within the $\mathrm{Cu}-\mathrm{O}$ planes, i.e., to $\mathrm{Cu}(2)$ ordering. Previous Mössbauer studies on $\left(\mathrm{Y}_{1-x} \mathrm{Pr}_{x}\right) \mathrm{Ba}_{2}\left(\mathrm{Cu}_{3-y} \mathrm{Fe}_{y}\right) \mathrm{O}_{z}[x=0,1 ; y-0 ; z=6,7]$ also revealed magnetic ordering of $\mathrm{Cu}(2),{ }^{2}$ but concluded that there was no overlap of superconductivity and antiferromagnetism. The present results suggest that spinglass-like magnetism coexists with superconductivity in the $\left(\mathrm{Y}_{1-x} \mathrm{Pr}_{x}\right) \mathrm{Ba}_{2} \mathrm{Cu}_{3} \mathrm{O}_{7}$ system. 


\section{Experimental Aspects}

Polycrystalline samples of $\left(\mathrm{Y}_{1-x} \mathrm{Pr}_{x}\right) \mathrm{Ba}_{2} \mathrm{Cu}_{3} \mathrm{O}_{7}(0 \leq x \leq 1)$ were prepared using conventional solid-state reaction techniques. ${ }^{1}$ High-resolution $\mathrm{x}$-ray diffraction studies indicated that the samples crystallized in an orthorhombic structure for all values of $x$. Both $\mathrm{x}$-ray and neutron-diffraction studies indicated that there were less than $2 \%$ impurity phases in all samples, and that phase separation ${ }^{3}$ of the constituents into $\mathrm{YBCO}$ with $T_{c}=90 \mathrm{~K}$ and $\left(\mathrm{Y}_{1-x} \mathrm{P}_{1_{x}}\right) \mathrm{Ba}_{2} \mathrm{Cu}_{3} \mathrm{O}_{7}$ of varying $x$ value with reduced $T_{c}$ did not occur. The effective moment determined from susceptibility measurements suggests that the Pr ions are close to tetravalent. We note, however, that other researchers using different experimental techniques have concluded that the $\operatorname{Pr}$ ions are trivalent. ${ }^{4}$

The ${ }_{1} \mathrm{SR}$ experiments were done at the stopped muon channel of the Los Alamos Clinton P. Anderson Meson Physics Facility (LAMPF) using standard zero-field techriques. ${ }^{5}$ The essential features of the technique are briefly described. Spin-polarized positive muons are implanted into a sample and the decay positrons are detected by counters located in the forward (direction of initia! muon momentum) and backward directions. In zero external field (nulled to $\pm 2 \mu \mathrm{T}$ ) the muon experiences only the internal magnetic field. Therefore, in an antiferromagnet one should observe one or more discrete frequencies determined by the magnitude of the local magnetic field at the muon site, i.e., $\omega_{\mu}=$ $\gamma_{\mu} H_{\text {loc }}\left(\gamma_{\mu} / 2 \pi=1.355 \mathrm{MHz} \mathrm{T}^{-1}\right)$.

\section{Results}

Representative zero-field $\mu$ SR spectra for $\mathrm{PrBa}_{2} \mathrm{Cu}_{3} \mathrm{O}_{7}$ taken at $300 \mathrm{~K}$ and $180 \mathrm{~K}$ are shown in Figs. 1(a) and 1(b). At $300 \mathrm{~K}$, the internal magnetic field is caused by randomly oriented, quasi-static $\mathrm{Cu}$ nuclear moments, which depolarize the muon according to $G(t)=\exp \left[-(1 / 2) \sigma^{2} t^{2}\right]$, where $\sigma$ is a Gaussian depolarization rate related to the second moment of the local field distribution at the muon site. The solid line of Fig. 1(a) is a Caussian fit to the data with $\sigma$ $=0.36 \mu \mathrm{s}^{-1}$. In contrast, the spectrum taken at $180 \mathrm{~K}$ shows oscillatory behavior with a well-defined muon frequency. This is clear evidence for the existence of an ordered, local magnetic field. The Fourier transform of Fig. 1(b) shows that the muon precessional frequency is $1.85 \mathrm{MHz}$. Similar results were obtained for $x=0.54,0.58,0.6,0.8$, and 1.0 . Muon precessional frequencies for $x=1.0,0.8$, and 0.6 are shown in Fig. 2. For clarity the $x=0.58$ and 0.54 results are not plotted. The Neel temperatures are approximately $285 \mathrm{~K}, 220 \mathrm{~K}, 35 \mathrm{~K}, 30 \mathrm{~K}$, and $20 \mathrm{~K}$ for $x=1,0.8,0.6,0.58$, and 0.54 , respectively.

From Fig. 2 we see that the zero-field $\mu$ SR frequencies appear to saturate at a value near $2.2 \mathrm{MHz}$, which corresponds to a local magnetic field of $16 \mathrm{mT}$. However, near $17 \mathrm{~K}(x=1.0)$, the local field is recluced to $\sim 12 \mathrm{mT}$. This reduction is attributed to the onset of Pr-moment ordering, as observed by magnetic susceptibility, ${ }^{1}$ specific heat, ${ }^{1}$ and neutron scattering. ${ }^{6}$ 


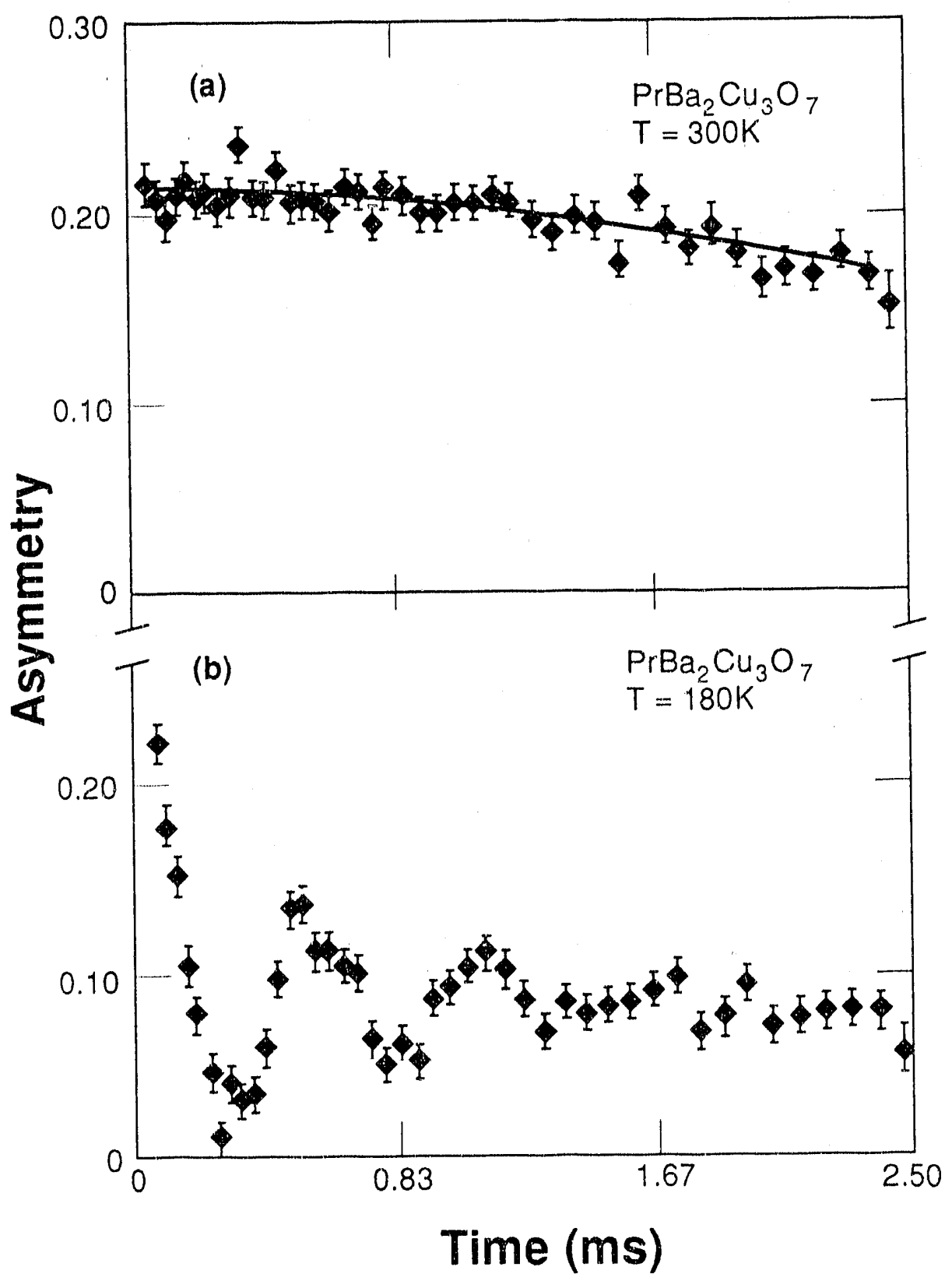

Fig. 1. Zero-field $\mu S R$ spectra for $\operatorname{PrBa}_{2} \mathrm{Cu}_{3} \mathrm{O}_{7}$ taken at (a) $300 \mathrm{~K}$ and (b) $180 \mathrm{~K}$. The oscillatory pattern in (b) is clear evidence for magnetic ordering. No ordering is evident in (a). 


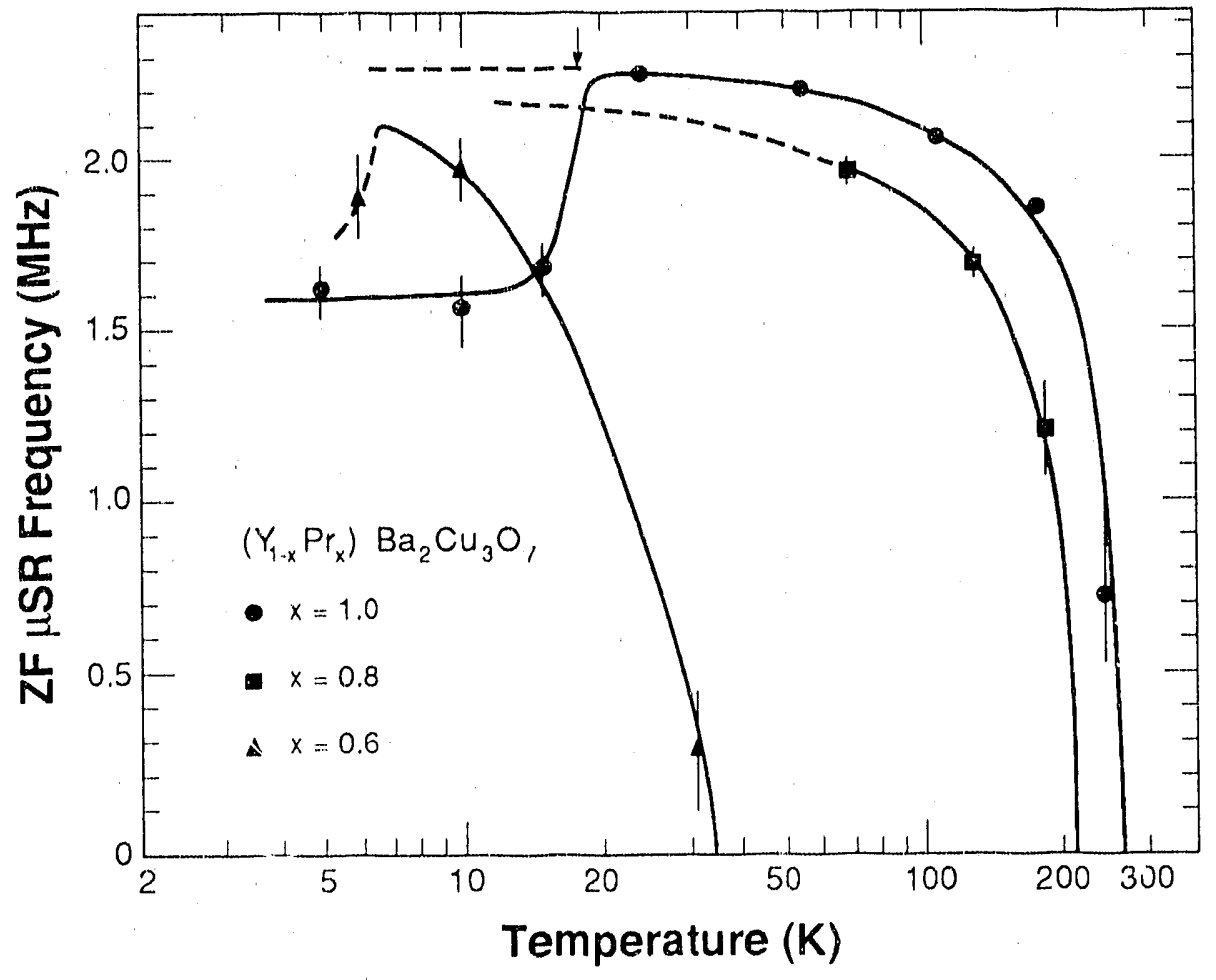

Fig. 2. Zero-field ${ }_{1} S R$ frequencies for $\left(Y_{1-x} \mathrm{Pr}_{5}\right) \mathrm{Ba}_{2} \mathrm{Cu}_{3} \mathrm{O}_{7}(x=1.0,0.8$, and $0.6)$ as a function of temperature. Néel temperatures are $285 \mathrm{~K}, 220 \mathrm{~K}$, and $35 \mathrm{~K}$, respectively.

The experimentally determined phase diagram for $\left(\mathrm{Y}_{1-r} \operatorname{Pr}_{x}\right) \mathrm{Ba}_{2} \mathrm{Cu}_{3} \mathrm{O}_{7}$ is shown in Fig. 3. $T_{N 1}$ is derived from zero-ficld $/ 1 S R$ data and is attributed to antiferromagnetic ordering of $\mathrm{Cu}(2) . T_{r}$ is based on susceptibility and resistivity measurements, and $T_{N 2}$ (associated with $\operatorname{Pr}$ ordering) is deduced from $/ \mathrm{SR}$, susceptibility, specific heat, and neutron scattering measurements. $/ S R$ data taken in a $100-\mathrm{m} T$ transverse field confirm the $T$, vs $x$ curve.

The region $0.4 \leq x \leq 0.6$ in the phase diagram is of special interest because it represents a crossover from magnetism to superconductivity. In Fig. 4 we show the muon depolarization for $x=0.6,0.58$, and 0.50 at a fixed temperature of $5 \mathrm{~K}$. It is clear that a well-defined frequency exists for $x=0.6$ and 0.58 , but not for $x$ $=0.5$. For the latler concentration it appears that the antiferromagnetic signature (i.e., a well-defined frequency) is absent. For $x=0.5$ there is a very fast-relaxing component of the muon signal in addition to a long-time tail. We attempted to fit the data of Fig. 4(c) with various known muon relaxation functions. The best fit [shown as the solid line of Fig. 4(c)] was obtained with a spin-glass function, 


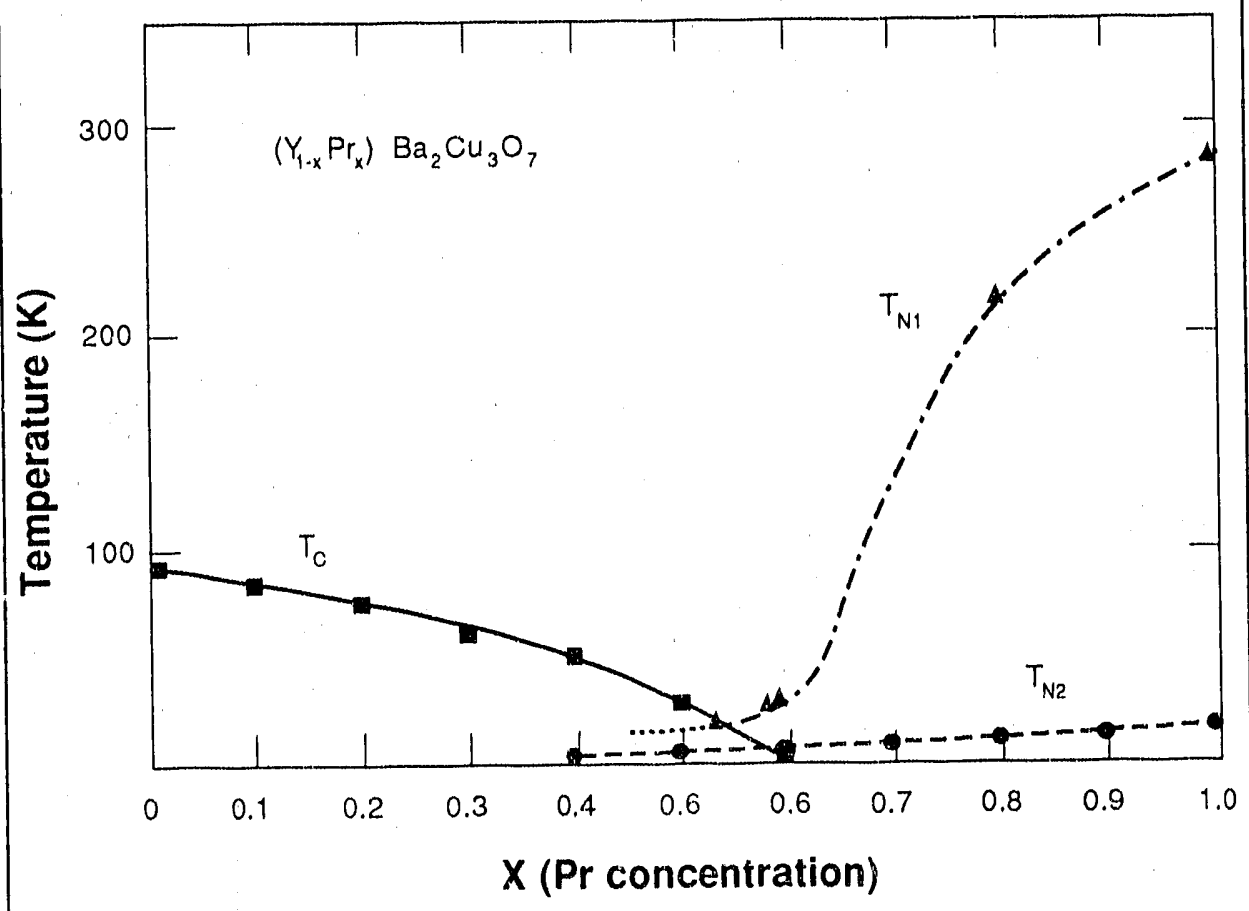

Fig. 3. Phase diagram for $\left(Y_{1-x} \operatorname{Pr}_{x}\right) \mathrm{Ba}_{2} \mathrm{Cu}_{3} \mathrm{O}_{3} . T_{N 1}$ corresponds to antiferromagnetic ordering of $\mathrm{Cu}$ moments within the $\mathrm{Cu}-\mathrm{O}$ planes as determined by zero-field $\mu . S R$ measurements. $T_{N 2}$ is attributed to Pr-moment ordering.

which assumed slow fluctuations of the time-varying local magnetic field, ${ }^{7}$

$$
i_{z}(t)=\frac{2}{3}\left(1-a_{0} t\right) \exp \left(-a_{0} t\right)+\frac{1}{3} \exp \left(\frac{-2 t}{3 \tau}\right) .
$$

In Eq. (1) $a_{0}$ is the width of the muon precession frequency distribution and $\tau$ is the correlation time. From the fitted data we obtained a fluctuation rate of $10^{5} \mathrm{~s}^{-1}$. However, based upon this fit we cannot conclude that the underlying magnetism is that of a spin glass, although it would not be unreasonable to assume that the crossover from antiferromagnetism to superconductivity produced a complex magnetic state that resembled a spin glass. This is especially true near $T=5 \mathrm{~K}$, where the Pr-moment ordering may also be contributing to the formation of this complex ground state, although the extrapolated Nél temperature for $x=$ 0.5 is only $3.4 \mathrm{~K}$.' Nevertheless, this region of the phase diagram presents a rich arena for investigating the interplay between magnetism and superconductivity in oxide superconductors. This is especially true for the $\left(\mathrm{Y}_{1-x} \operatorname{Pr}_{x}\right) \mathrm{Ba}_{2} \mathrm{Cu}_{3} \mathrm{O}_{7}$ system because it exhibits both magnetism and superconductivity, and retains the orthorhombic structure for $0 \leq x \leq 1$. 


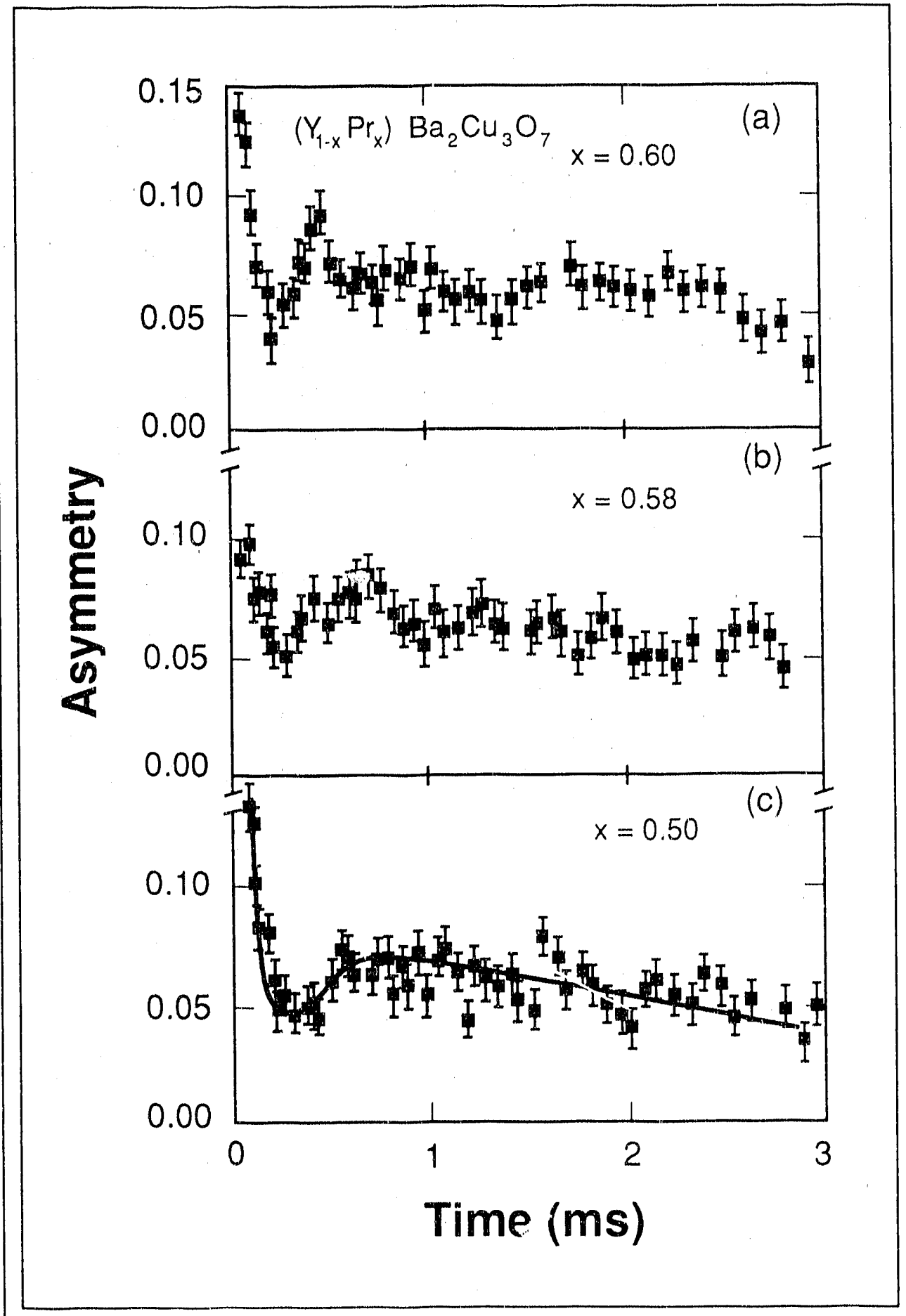

Fig. 4. Zero-field $\mu \mathrm{SR}$ spectra for $\left(\mathrm{Y}_{!_{-x}} \mathrm{Pr}_{x}\right) \mathrm{Ba}_{2} \mathrm{Cu}_{3} \mathrm{O}_{7}$ taken at $5 \mathrm{~K}$; (a) $x=0.6 ;(b) x=0.58$; and (c) $x=0.50$. 
Based on our $/ 1 S R$ data, we conclude that no clear signature of antifer romagnet: 0 m exists in $\left(Y_{1-x} P_{x}\right) \mathrm{Ba}_{2} \mathrm{Cu}_{3} \mathrm{O}_{7}$ for a concentration of $x=0.5$. We show a dot-dashed line in the phase diagram (Fig. 3) to represent this fact. Thus, the conclusion that antiferromagnetism and superconductivity do not coexist in this compound, as offered by Felner et al., ${ }^{2}$ is in agreement with our observations. On the other hand, we suggest that spin-glass-like magnetism and superconductivity do indeed coexist.

Zcro-field ${ }_{\mu} \mathrm{SR}$ data taken on $\mathrm{PrBa}_{2} \mathrm{Cu}_{3} \mathrm{O}_{6}$ show that magnetic ordering occurs above room temperature, which is the highest operating temperature of our spectrometer. By extrapolating the available data we estimate $T_{N} \sim 325 \mathrm{~K}$. Interestingly, two distinct frequencies are observed in this system, which we attribute to the existence of two magnetically nonequivalent muon stopping sites. At $250 \mathrm{~K}$ and $15 \mathrm{~K}$, the measured muon precessional frequencies are $\nu_{1}=1.5 \mathrm{MHz}$ and $\nu_{2}$ $=3.5 \mathrm{MHz} ; \nu_{1}=2.2 \mathrm{MHz}$ and $\nu_{2}=4.7 \mathrm{MHz}$, respectively.

In summary, zero-field $\mu \mathrm{SR}$ experiments have been conducted on $\left(\mathrm{Y}_{1-x} \mathrm{Pr}_{x}\right) \mathrm{Ba}_{2} \mathrm{Cu}_{3} \mathrm{O}_{7}$ as a function of concentration and temperature. Clear evidence for antiferromagnetic ordering of the $\mathrm{Cu}$ moments within the $\mathrm{Cu}-\mathrm{O}$ planes is found. Néel temperatures vary from $285 \mathrm{~K}$ to $20 \mathrm{~K}$ for $x=1.0$ and 0.54 , respectively. Coexistent spin-glass-like magnetism and superconductivity (in addition to the $\mathrm{Pr}$ magnetism) occur for $x=0.5$. $\mathrm{PrBa}_{2} \mathrm{Cu}_{33} \mathrm{O}_{6}$ is antiferromagnetic with an estimated Nécl temperature of $325 \mathrm{~K}$.

\section{References}

1. A. Kebede, C. S. Jee, J. Schwegler, J. E. Crow, T. Mihalisin, G. H. Myer, R. E. Salomon, P. Schlottmann, M. V. Kuric, S. H. Bloom, and R. P. Guertin, Phys. Re., B 40, 4453 (1989).

2. I. Felner, U. Yaron, I. Nowik, E. R. Bauminger, Y. Wolfus, E. R. Yacoby, G. Hilscher, and N. Pillmayr, Phys. Rev. B 40, 6739 (1989).

3. H. B. Radousky, K. F. McCarty, J. L. Peng, and R. N. Shelton, Phys. Rev. B 39, 12.383 (1989).

4. M. E. Lopez-Morales, D. Rios-Jara, J. Tagena, R. Escudero, S. La Placa, A. Bezinge, V. Y. Lce, E. M. Engler, and P. M. Grant, Phys. Rev. B 41, 6655 (1990).

5. A. Schenck, Muon Spin Rotation Spectroscopy (Adam Hilger, Bristol, 1986).

6. W.-H. Li, J. W. Lynn, S. Skanthakumar, T. W. Clinton, A. Kebede, C.-S. Jee, J. E. Crow, and T. Mihalisin, Phys. Rev. B 40, 5300 (1989).

7. R. H. Heffner, M. Leon, M. E. Schillaci, D. E. MacLaughlin, and S. A. Dodds, J. Appl. Phys. 53, 2174 (1982). 
Nuclear Chemistry

EXPERIMENT 1100 - TOFI

\section{Direct Mass Measurements Using the TOFI Spectrometer: The Neutron-Rich Isotopes of Chlorine through Iron}

Utah State Univ., Los Alamos, Nanjing Univ. (PRC), Univ. of Giessen (W. Germany)

\section{Spokesman: J. M. Wouters (Los Alamos) \\ Participants: X. L. TU, X. G. Zhou, D. J. Vieira, J. M. Wourers, Z. Y. Zhou, H. L. Selfert, and V. G. Lind}

\section{Introduction}

Nuclear masses have been of fundamental importance to nuclear science ever since J. J. Thompson used them to reveal the existence of multiple isotopes for a given element in 1913. In present day experiments nuclear masses are used:

1. To examine the basic properties of nuclei such as pairing interactions, Coulomb effects, and changes in nuclear structure;

2. To probe new regions of nuclei where large-scale changes occur in shape; and

3. As input parameters in a variety of theoretical calculations including astrophysical models.

This greatly expanded role has arisen naturally because the mass of a nucleus directly reflects the sum of the forces that hold a nucleus together. The key to using masses as described above is to measure them in a systematic fashion and to look for global as well as local variations in the mass surface. These measurements are especially important far from the valley of $\beta$-stability where the mass is often the first piece of quantitative information to be determined-.thus leading to new insight into the nature of such nuclei with extreme neutronto-proton ratios. Such was the case for our recent measurements in the ${ }^{24} \mathrm{O}$ region where a dramatic confirmation of shell-model wave functions and twobody interaction strengths was shown. ${ }^{1}$

The advent of the recoil mass spectrometer has greatly facilitated experiments to measure masses on the neutron-rich side of the vallcy of $\beta$-stability. Such systems have distinct advantages because they are universal, fast, and require little or no excited state level information about the nucleus being studied. During the last fow years, the experiments of our group and that of a French group have used recoil mass spectrometers to determine the masses of approximately 30 neutron-rich isotopes ranging from ${ }^{11} \mathrm{Li}$ to ${ }^{3 \pi} \mathrm{P}, 1,2,3$ In this report, we present our most recent experimental results on the masses of several neutron-rich isotopes of chlorine through iron. Our motivation for performing this experiment was to use the large number of new masses made available to investigate:

1. The systematic behavior of the pairing energy;

2. The location of a new region of enhanced binding; and

3. The checking of the validity or failure of a variety of mass models.

\section{Experimental Procedure}

Our ability to make direct mass measurements of exotic neutron-rich nuclei relies on the use of a unique Time-of-Flight Isochronous (TOFI) recoil spectrometer. This spectrometer can measure the masses of fast recoiling nuclei that are 
produced at the Los Alamos Meson Physics Facility through interaction of the $800-\mathrm{MeV}, 1-\mathrm{mA}$ proton beam with a thin $\left(1 \mathrm{mg} / \mathrm{cm}^{2}\right){ }^{\text {nat }} \mathrm{Th}$ target. TOFI's unique feature is its isochronous design which requires that ions with the same massto-charge ratio take the same amount of time to pass through the spectrometer independent of their velocities. The corollary to this velocity independence is that a precise measurement of an ion's time-of-flight is a direct measurement of the ion's mass-to-charge ratio la typical resolution for this experiment was $\Delta(\mathrm{m} / \mathrm{q}) /(\mathrm{m} / \mathrm{q})=2.3 \times 10^{-4}$ (FWHM)]. The charge state and atomic number of each recoil were determined from additional measurements of the ion's velocity, stopping power, and total kinetic energy. Advantages of measuring masses using TOFI beyond those already discussed for recoil mass spectrometers in general include:

1. The need to measure only one parameter with high precision; and

2. The distributions of the knowns and unknowns (largely intermixed in the mass-to-charge spectrum such that no extrapolations of the calibration are needed).

\section{Results}

We have measured the masses of over 35 neutron-rich nuclei ranging from ${ }^{45} \mathrm{Cl}$ to ${ }^{66} \mathrm{Fe}$. Given these new measurements, we have extended the mass surface by as many as five neutrons for a given element and compared the shape to theory; the differences lead to several new insights.

Several groups have suggested that there is an isospin dependence in the neutron and proton pairing energies. ${ }^{4,5}$ Our data enabled us to examine this dependence in a region where sudden changes in the nuclear structure from one nucleus to the next are expected to be minimal. Although additional measurements are needed, our work leads to a confusing result where we observe some isospin dependence in the neutron pairing energies, but little or no dependence in the proton pairing energies.

A plot of the two-neutron separation energies $\left[S_{2 n}=M(A-2, Z)+2 N-\right.$ $M(A, Z)$ ] derived from our mass measurements is shown in Fig. 1. Two-neutron separation energies enable one to highlight (model independently) local structural changes without the odd-even effects caused by nuclear pairing. The most striking feature in Fig. 1 is the sudden decrease in $S_{2 n}$ just after the $N=28$ closed shell. Of further interest is the leveling off of both the calcium and scandium $S_{2 n}$ lines at $N=31$ (i.c., ${ }^{51} \mathrm{Ca}$ and ${ }^{52} \mathrm{Sc}$ ). This trend continues out to ${ }^{53} \mathrm{Sc}, N=32$, but is not as evident in ${ }^{52} \mathrm{Ca}$. Such a change in slope is reminiscent of the $S_{2 n}$ upturn observed at $N=20$ for neutron-rich sodium isotopes that indicated the onset of prolate deformation. Another, but less likely explanation, is the occurrence of a neutron subshell closure at $N=32$. We would expect ${ }^{52} \mathrm{Ca}$ to show the largest effect from such a subshell closure since it would be supported by $Z=20$ shell closure; however, we do not observe this effect. 


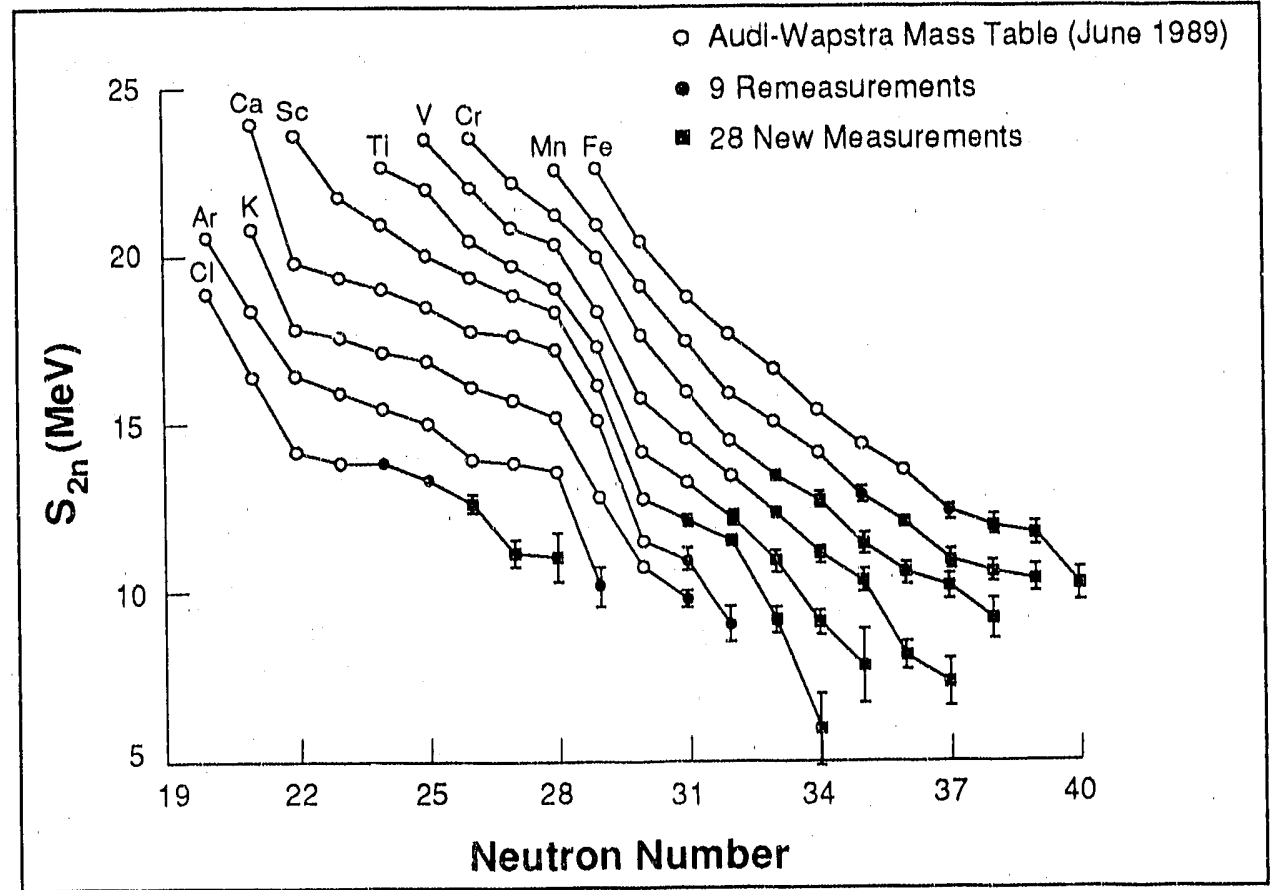

Fig. 1. Plot of two-neutron separation energies $S_{2 n}$ vs neutron number. The open circles represent previous measurements, the solid circles indicate nuclei which have been remeasured, and the solid squares are for nuclei where firsttime measurements are reported herein.

Finally, we have compared our data to several mass models (sce Fig. 2). On the whole, the agreement between our data and theory is good. One area of discrepancy occurs for the calcium and scandium isotopes near $N=32$ where theory consistently predicts too little binding. Again this effect is more pronounced in the scandium isotopes and suggests that a local deformation is occurring in these nuclei. Another discrepancy worth noting is the tendency for most theories to overpredict the binding of neutron-rich nuclei above titanium. This discrepancy tends to grow with increasing atomic number as well as with neutron-excess, although the deviations between theory and experiment rarely exceed $3 \sigma$ experimental standard deviations.

\section{Conclusion}

Nuclear masses continue to be an important source of information about the interplay of forces within a nucleus and the resulting nuclear structure effects they elicit. In this annual report, we have shown how mass measurements of the very neutron-rich isotopes of chlorine through iron have been used to specifically study pairing energy systematics, to define a new region of enhancing binding 


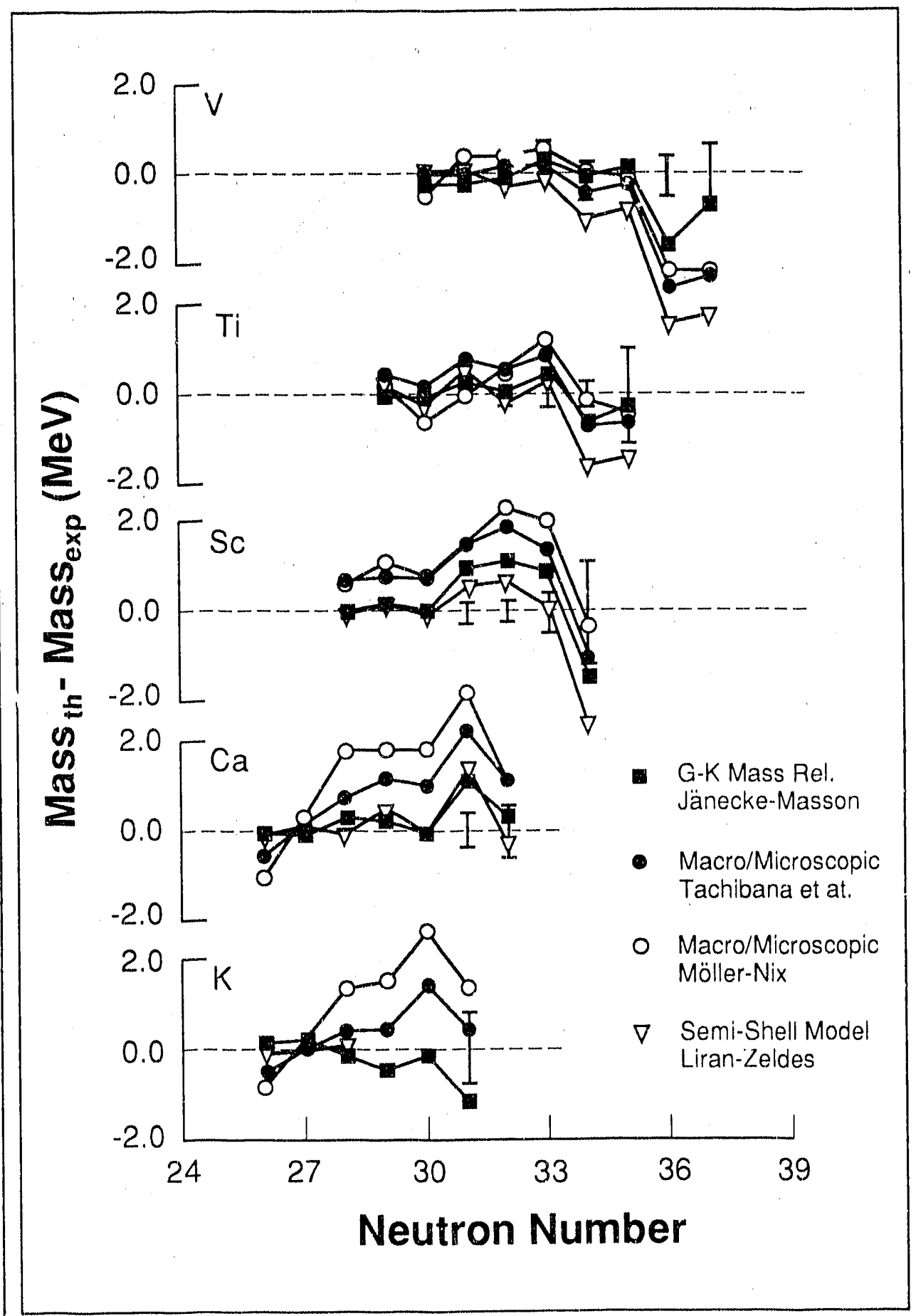

Fig. 2. Comparison of the mass predictions of Möller-Nix, Tachibana, LiranZeldes, and Jänecke-Masson with the experimental masses. ${ }^{6}$ The experimental error bars are shown for our data. 
caused by changes in nuclear structure/shape, and to evaluate several mass models. Improvements to TOFI and the detection system are planned in order to extend our measurements to both higher $Z$ elements and to more neutron-rich nuclei. With more extensive measurements and refined theoretical models, we will continue to advance our knowledge of the atomic mass surface.

\section{References}

1. J. M. Wouters, R. H. Kraus, D. J. Vieira, G. W. Butler, and K. E. G. Löbner, Z. Phys. A 331, 229 (1988).

2. D. J. Vieira, J. M. Wouters, K. Vaziri, R. H. Kraus, H. Wollnik, G. W. Butler, F. K. Wohn, and A. H. Wapstra, Phys. Rev. Lett. 57, 3253 (1986).

3. A. Gillibert et al., Phys. Lett. 192B, 39 (1987); A. Cillibert et al., Phys. Lett. 176B, 317 (1986).

4. P. Vogel, B. Jonson, and P. G. Hansen, Phys. Lett. 139B, 227 (1984); A. S. Jensen, P. G. Hansen, B. Jonson, Nucl. Phys. A431, 393 (1984).

5. D. G. Madland and J. R. Nix, Nucl. Phys. A476, 1 (1988).

6. P. Möller and J. R. Nix, At. Data and Nucl. Data Tables 39, 213 (1988); T. Tachibana, M. Uno, M. Yamada, and S. Yamada, At. Data and Nucl. Data Tables 39, 251 (1988); S. Liran and N. Zeldes, At. Data and Nucl. Data Tables 17, 431 (1976); J. Jänecke and P. J. Masson, At. Data and Nucl. Data Tables 39, 265 (1988). 


\section{Radiation Effects}

\section{EXPERIMENT 1014 - Radiation-Effects Facility \\ Proton, Spallation- Neutron, and Fission- Neutron Irradiation of Copper}

\author{
Risø National Laboratory, \\ Denmark; Los Alamos
Spokesmen: A. Horsewell (Ris National Laboratory, Denmark) and W. Sommer (Los Alamos)

Participants: B. N. Singh, T. Leffers, and M. Eidrup

\section{Objectives}

The objectives of this project are concerned with furthering our basic understanding of the mechanisms of radiation damage in metals.

Varying the type and energy of the incident particle from 1-MeV fission neutrons to $600-\mathrm{MeV}$ protons results in the average energy of the primary knock-on atoms (PKA) being varied by over two orders of magnitude from $10^{4}$ to $10^{6} \mathrm{eV}$. The number and spatial distribution of the displaced atoms that result from each colliding incident particle are not known for very high-energy PKA reccils. Also, transmutation element production increases markedly for the higher-energy incident particles; this is of concern both because of microstructural modification due to the interaction of impurities with cascade damage regions and because of the general problem of activation.

The range of PKA energies used for correlation in this work spans that expected for fusion reactor components and is therefore of relevance to fusion reactor materials.

\section{Achievements in 1989}

Achievements during 1989 included analysis of high-purity copper irradiated to fluences in the range $5 \times 10^{23}-5 \times 10^{25} \mathrm{p}^{+} / \mathrm{m}^{2}$ at $313-323 \mathrm{~K}$ at LAMPF Target $\mathrm{A} 6$ by high-resolution weak beam electron microscopy at Ris $\phi$. Clusters of vacancies (Stacking Fault Tetrahedra) and interstitials (interstitial loops) have been observed at high density $\left(\sim 10^{24}\right.$ defects $\left./ \mathrm{m}^{3}\right)$ having sizes of $0.5-6 \mathrm{~nm}$ in diameter. Such defect clusters are characteristic of collapsed cascade damage regions resulting from primary and secondary recoil events. The observations of LAMPF specimens have been complimented to lower fluences using copper and gold specimens irradiated by $600-\mathrm{MeV}$ protons in the PIREX facility at the Paul Scherrer Institute (PSI) in Switzerland, where the irradiations were carried out at $323 \mathrm{~K}$ in the fluence range $10^{21}$ to $10^{24} \mathrm{p}^{+} / \mathrm{m}^{2}$.

The microstructures resulting from medium-energy proton irradiations have been compared to that of similar specimens irradiated with fast neutrons (DR-3 at Ris $\phi$ and in the Omega West Reactor at Los Alamos) and with $14-\mathrm{MeV}$ neutron irradiated specimens (Rotating Target Neutron Source II, Lawrence Livermore Laboratory).

The major conclusions of this intercomparison are:

1. Collapsed cascade defect cluster concentrations increase in direct proportion to fluence up to $10^{24} \mathrm{p}^{+} / \mathrm{m}^{2}$; saturation in density occurs at $\sim 10^{24}$ defect clusters $/ \mathrm{m}^{3}$.

2. Defect cluster densities are approximately one order of magnitude lower per incident particle when compared to fast $(1 \mathrm{MeV})$ and $14-\mathrm{MeV}$ neutron irradiations. 
3. The size distributions of defect clusters are broadly comparable for all irradiations studied in copper; however, proton-irradiated gold shows significantly larger average defect cluster size.

We interpret these observations as lending support to the model of very high recoil energies being dissipated through the subdivision of recoil energy into subcascades. Within this framework, there is little structural difference between cascades and subcascades. However, the results for the gold specimens indicate that fission events may be important in medium-energy proton irradiation of high $Z$ materials.

Work is in progress to extend this study to other LAMPF A6 protonirradiated pure metals and alloys, as well as to LASREF spallation neutronirradiated specimens.

\section{Publications}

A. Horsewell, B. N. Singh, S. Proennecke, W. F. Sommer, and H. L. Heinisch, "Defect Structures in Copper and Gold Irradiated with Fast Neutrons, 14-MeV Neutrons, and 600-800-MeV Protons," presented at the 4th International Conference on Fusion Reactor Materials, Kyoto, Japan, December 1989 [to be published in Journal of Nuclear Materials (1990)]. 
EXPERIMENT 1139 - Beam Stop Irradiation Facility

Testing of Radiation Resistance of ReadOut Chips for Use in High-Rate Nuclear and Particle Physics Applications

Los Alamos, UC Santa Cruz, Univ. of Missouri, INFN Torino

Spokesmen: C. M. Hottman, W. F. Sommer, H. J. Zlock (Los Alamos), and H. F.W. Sadrozinskl (UC Santa Cruz)

Participants: C. M. Hotfman, D. Holtkamp, W. W. Kinnison, $C$. Milner, W. F. Sommer, H. J. Zlock, J. Bacigalupl, N. Cartiglia, J. DeWint, A. Kakuzniacki, H. Koianoskl, D. Pitzl, W. A. Rowe, H. F.W. Sadrazinskl, $E$. Spencer, $P$. Tonobsum, $P$. Ferguson, P. Giubellino, and $S$. Sartori
One of the main requirements for detectors and front-end electronics at the Superconducting Super Collider (SSC) is that they be radiation resistant. It is expected that this electronics will be subject to a yearly radiation dose of $0.3 \mathrm{MRad}$ caused by charged particles and a yearly fluence of $10^{12}$ neutrons $/ \mathrm{cm}^{2}$. As part of our program to develop a silicon tracking device for the $5 \mathrm{SC}$, we have exposed radiation-hard CMOS transistors and PIN diodes to the $800-\mathrm{McV}$ proton beam and to neutrons at the beam stop at LAMPF. We measured the leakage current constants for the diodes and threshold voltage shifts for the transistors under varying biasing conditions.

At the recent European Symposium on Semiconductor Devices, we reported the results of the irradiation of $n$ and $\mu$ transistors on $2-\mu \mathrm{m}$ CMOS test structures with neutrons at the LAMPF neutron spallation source. This past year we have extended our measurements to irradiation by $800-\mathrm{MeV}$ protons and by much higher neutron fluxes. We paid particular attention to accurate measurements of the fluenzes.

For digital application, the radiation damage to CMOS transistors is characterized by a shift of the threshold voltage, $V_{h h}$, the gate voltage at which the transistor turns on. We concentrated our tests on transistors of $1.2-\mu \mathrm{m}$ feature size produced by United Technologies Microelectronics Center, which we showed were particularly radiation-resistant. Figure 1 shows the threshold voltage shifts for a typical $n$ - and $y$-transistor during and 22 days following proton irradiation. One can clearly see the saturation effect during exposure and a rebound during annealing after the exposure. The results show that the UTMC process is sufficiently radiation resistant for application at any contemplated accelerator.

We also tested PIN photodiodes, Ha mamatsu \#S1723, to simulate the effects on silicon strip detectors. The increase in leakage current was measured and found to be independent of proton flux and whether or not the diode was biased during irradiation. We also iound evidence for annealing following irradiation with a time constant of $124 \pm 36$ days but that the annealing stops after the current reaches $50 \%$ of the initial current.

\section{Publications and Papers}

H. J. Ziock et al., "Measurement of Proton-Induced Radiation Damage to CMOS Transistors and PIN Diodes," IEEE Trans. Nucl. Sci. 37, 1238 (1990). 


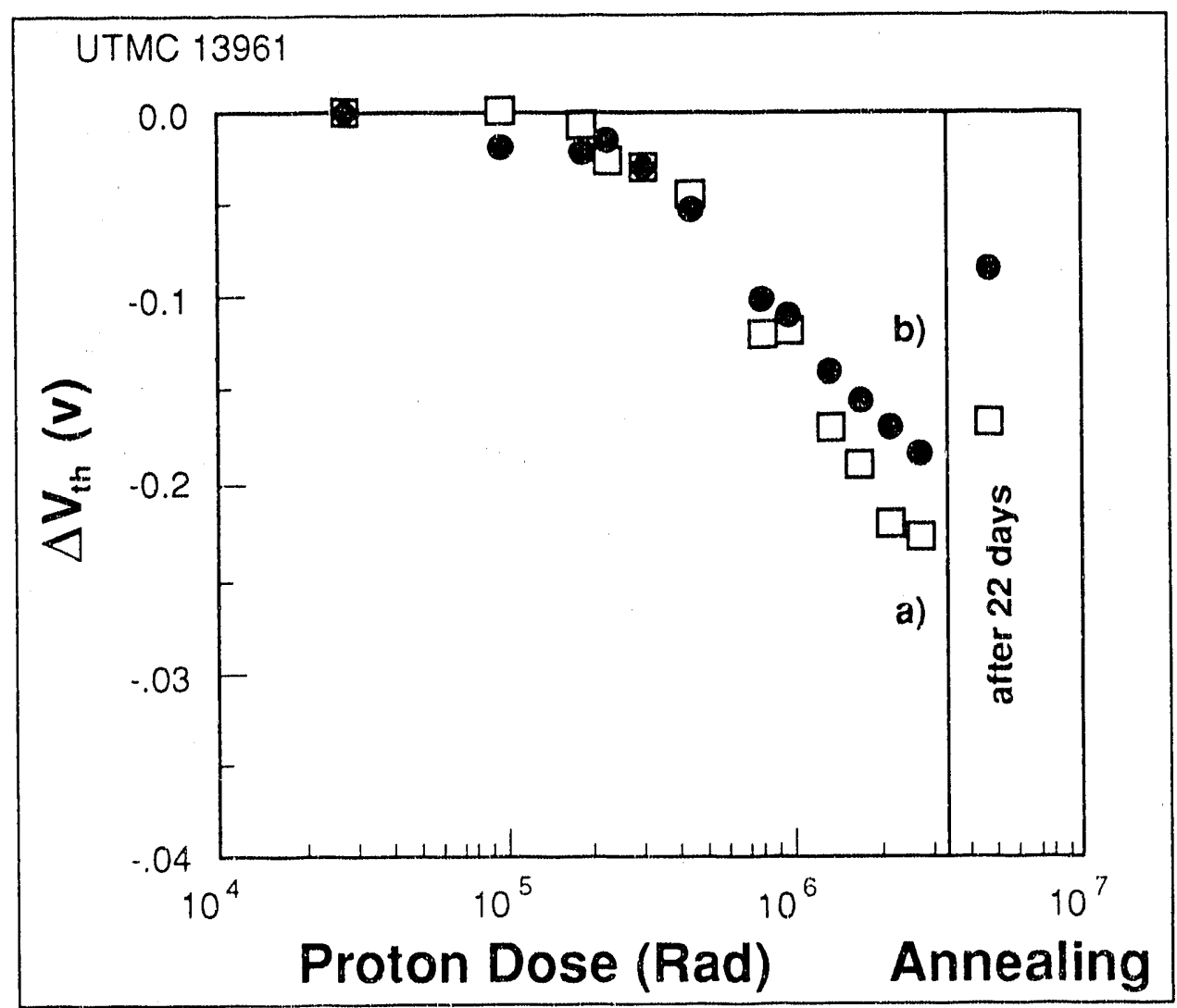

Fig. 1. Threshold voltage shifts, $\Delta V_{\text {th }}^{\prime}$ for UTMC transistors as a function of fluence: (a) $p$-transistor, (b) n-transistor. Also shown are the results after unbiased room-temperature annealing of 22 days. 
Radiation Effects

EXPERIMENT 1165 -Spallation Radiation Effects Facility

\section{Synergetic Load Effects on the Beam Entry Window of a High-Power Spallation Neutron Source}

PSI (Switzerland), LoS Alamos

Spokesman: G. S. Bauer (PSI)

Participants: G. S. Baver, M. Dubs, W. E. Fischer, M Victorla, and W. F. Sommer
The upgraded spallation neutron source at the Paul Scherrer Institute (PSI) accelerator requires a metal window to separate the vacuum chamber from the liquid $\mathrm{Pb}-\mathrm{Bi}$ target material. As a test of the window design, two full-scale windows were installed at Target Station A-6, attached to the proton inserts that are part of the Los Alamos Spallation Radiation Effects Facility. The proton current density here is similar to that expected at PSI. The candidate material is a ferritic-martensitic stainless steel $\left(\mathrm{Fe}^{12} \mathrm{Cr} \mathrm{ro}^{1} \mathrm{Mo}\right.$ ) and the design incorporates two hemispherical shells cooled by an internal flow of water.

During 1989, one window received 1.2 A-h and the second received $0.9 \mathrm{~A}-\mathrm{h}$ of beam. The goal of 2 A-h should be reached during 1990. After the irradiation is complete, the windows will be returned to PSI for mechanical property tests and transmission elertron microscopy analysis. 
RESEARCH

Radioisotope Production

\section{Radioisotope Production}

\section{INC-11 Radioisotope Production Activities}

P. Chamberlin, A. H. Herring, R. M. Lopez, B. Garcia, M. Garcia, R. Gritzo, P. S. Lysaght, S. Maestas, R. A. Mitchell, M. A. Montoya, D. C. Moody, M. A. Ott, C. P. Padilla, M. Peeples, G. Peterson, D. R. Phillips, L. Schneider, R. C. Staroski, F. J. Steinkruger, W. A. Taylor, and D. H. Vigil

\section{Stable and Radioactive Isotope Production and Separation}

A significant portion of the Group INC-11 Radioisotope program involves producing and shipping radioisotopes for the medical research community. These radioisotopes are generally unavailable commercially or can be made in high yields only at LAMPF. Group INC-11 provides these radioisotopes on a costrecovery basis to interested researchers. During FY1989, we shipped isotopes to a total of 44 organizations around the world and to four groups at Los Alamos, including our own Medical Radioisotopes Research Program (see Table I).

Table I. Medical Radioisotope Shipments.

\begin{tabular}{|c|c|c|c|c|}
\hline Isotope & Customer & $\begin{array}{l}\text { No. of } \\
\text { Shipments }\end{array}$ & $\begin{array}{l}\text { Shipped } \\
\quad(m C i)\end{array}$ & $\begin{array}{l}\text { Received } \\
\quad(m C i)\end{array}$ \\
\hline${ }^{26} \mathrm{Al}$ & TRIUMF, Canada & 1 & 0.10 & 0.10 \\
\hline${ }^{73}$ As & $\begin{array}{l}\text { Battelle } \\
\text { U.S. Bureau of Mines } \\
\text { U.S. Geological Survey } \\
\text { Univ. of California, Davis } \\
\text { Univ. of California, } \\
\text { Riverside } \\
\text { Univ. of Arizona } \\
\text { Univ. of Central Florida } \\
\text { Wayne State Univ. }\end{array}$ & 9 & 63.63 & 63.00 \\
\hline${ }^{7} \mathrm{Be}$ & $\begin{array}{l}\text { Amcrsham International, } \\
\text { UK }\end{array}$ & 1 & 20.00 & 20.00 \\
\hline${ }^{207} \mathrm{Bi}$ & Isotope Products Labs & 1 & 41.00 & 41.00 \\
\hline${ }^{109} \mathrm{Cd}$ & $\begin{array}{l}\text { E. I. Dupont/NEN } \\
\text { Products } \\
\text { Isotope Products Labs } \\
\text { Los Alamos/INC-11 } \\
\text { Los Alamos/P-10 }\end{array}$ & 13 & 2041.10 & 2035.00 \\
\hline${ }^{64} \mathrm{Cu}$ & Los Alamos/INC-11 & 1 & 193.10 & 193.10 \\
\hline${ }^{67} \mathrm{Cu}$ & $\begin{array}{l}\text { California State } \\
\text { Univ., Fullerton } \\
\text { IBM Research Center } \\
\text { Los Alamos/INC-11 } \\
\text { Los Alamos/LS-3 }\end{array}$ & 68 & 3426.75 & 2860.16 \\
\hline
\end{tabular}




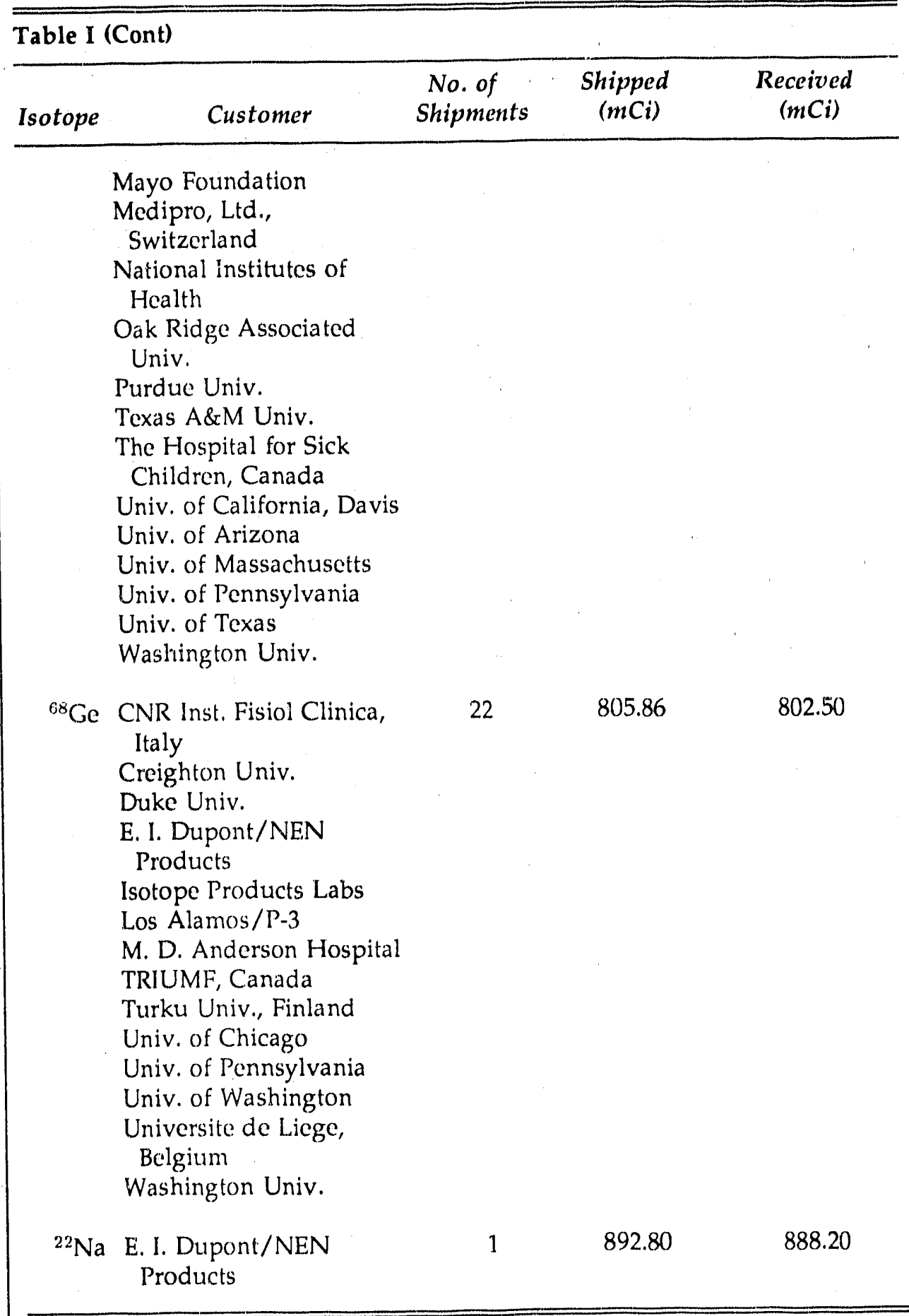




\begin{tabular}{|c|c|c|c|c|}
\hline \multicolumn{5}{|c|}{ Table I (Cont) } \\
\hline Isotope & Customer & $\begin{array}{l}\text { No. of } \\
\text { Shipments }\end{array}$ & $\begin{array}{l}\text { Shipped } \\
\quad(m \mathrm{C} i)\end{array}$ & $\begin{array}{l}\text { Received } \\
\quad(m C i)\end{array}$ \\
\hline${ }^{83} \mathrm{Rb}$ & $\begin{array}{l}\text { Los Alamos/P-3 } \\
\text { Univ. of California, } \\
\text { Lawrence Livermore } \\
\text { National Laboratory }\end{array}$ & 4 & 91.20 & 91.00 \\
\hline${ }^{82} \mathrm{Sr}$ & $\begin{array}{l}\text { E. R. Squibb \& Sons } \\
\text { Nordion, Canada } \\
\text { Univ. of California, } \\
\text { Lawrence Berkeley } \\
\text { National Laboratory }\end{array}$ & 13 & 7546.77 & 7320.67 \\
\hline${ }^{48} \mathrm{~V}$ & Univ. of Chicago & 1 & 1.04 & 1.00 \\
\hline${ }^{127} \mathrm{Xe}$ & $\begin{array}{l}\text { Brookhaven National } \\
\text { Laboratory }\end{array}$ & 1 & $\begin{array}{c}2.40 \\
\text { (Curies) }\end{array}$ & $\begin{array}{c}2.40 \\
\text { (Curies) }\end{array}$ \\
\hline${ }^{8 B} Y$ & $\begin{array}{l}\text { Abbott Labs } \\
\text { Amersham International, } \\
\text { UK } \\
\text { Analytics, Inc. } \\
\text { Bionetics Research } \\
\text { Centocor } \\
\text { Isotope Products Labs } \\
\text { National Institutes of } \\
\text { Health } \\
\text { Univ. of California, Davis } \\
\text { Univ. of California, } \\
\text { Davis Chemistry }\end{array}$ & 10 & 47.51 & 47.00 \\
\hline${ }^{8} \mathrm{Zr}$ & $\begin{array}{l}\text { Argonne National } \\
\text { Laboratory }\end{array}$ & 1 & 5.00 & 5.00 \\
\hline & TOTAL & 147 & 15178.36 & 14368.37 \\
\hline
\end{tabular}




\section{Theory}

Configuration Mixing as a Source of ThreeBody Molecular Formation in MuonCatalyzed $d \cdot t$ Fusion

M. Leon (Los Alamos)
Recent experiments at LAMPF $^{1}$ and Paul Scherrer Institute (PSI) ${ }^{2}$ have provided evidence that the rate for resonant molecular formation,

$$
t \mu+\left(D_{2}\right)_{0, K_{1}} \rightarrow\left[(d t \mu)_{11} d 2 c\right]_{\nu_{1} K_{j}}^{*},
$$

which plays a central role in the $d-t$ catalysis cycle, ${ }^{3}$ contains density dependence beyond that appropriate to two-body collisions. In a recent paper, ${ }^{4}$ it has been demonstrated that configuration mixing of the angular momentum $\left(K_{j}\right)$ states of the $\left[(d t \mu)_{11} d 2 r\right]_{\nu, K}^{*}$, complex formed in reaction (1), caused by the proximity of a neighboring molecule, produces a significant amount of three-body molecular formation. This occurs because the center-of-mass of the complex is appreciably displaced from its geometrical center, so that the neighbor exerts a torque on the complex; this results in the " $\nu, K_{f}^{\prime}$ " energy eigenstates not being angular momentum eigenstates.

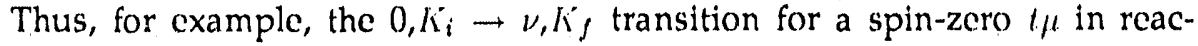
tion (1) with $K_{i}=0, \nu=2$, and $K_{j}=2$ is believed to have a transition energy close to zero ${ }^{3}$ and thus to be dominant for low-collision energies. Then, a nearby neighboring molecule implies that the " $K_{j}=2$ " energy eigenstate has small components with $K_{j}=1$ and $K_{j}=3$. The $K_{j}=1$ component will then contribute to the transition matrix element for reaction (1) with an $L=0$ spherical Bessel function instead of $L=1$, which to a large extent compensates for the smallness of the mixing coefficient. ${ }^{4}$ Molecular formation rates for reaction (1) calculated using this idea for three choices of $0 \rightarrow 2(\nu=2)$ transition energies are shown in Fig. $1 .^{4}$ The three-body molecular formation produced in this way may be sufficient to explain the observed effect.

\section{References}

1. S. E. Jones, A. N. Anderson, A. J. Caffrey, C. DeW. Van Siclen, K. D. Watts, J. N. Bradbury et al., Phys. Rev. Lett. 56, 588 (1986).

2. C. Petitjean, P. Ackerbauer, W. H. Breunlich, M. Cargnelli, M. Jeitler, P. Kammel et al., Muon Catal. Fusion 2, 37 (1988).

3. W. H. Breunlich, P. Kammel, J. S. Cohen, and M. Leon, Ann. Rev. Nucl. Part. Sci. 39, 311 (1989).

4. M. Lcon, Phys. Rev. A 39, 5554 (1989). 


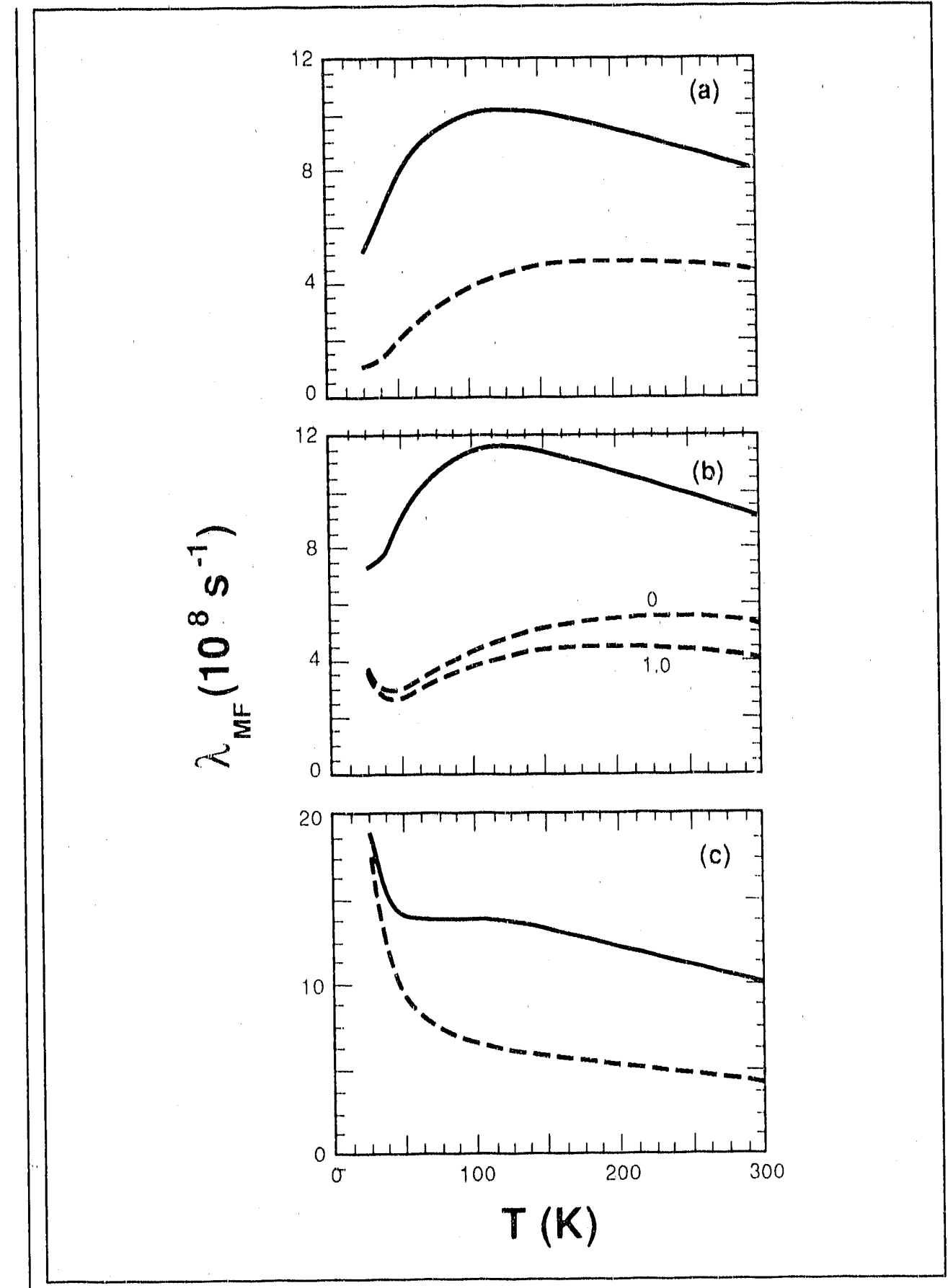

Fig. 1. Two-body (solid curve) and three-body (dashed curve, $\left.x \phi^{-1}\right)$ molecular formation rates for reaction (1) ( $\phi$ is the density in units of liquid $H_{2}$ density). Transition energies have been chosen to correspond to $0 \rightarrow 2$ falling at (a) $-2.0 \mathrm{meV} ;(b)-0.5 \mathrm{meV} ;(c)+1.0 \mathrm{meV}$. For further details, see Ref. 4. 


\section{Neutron Radius Analysis in the Trinucleon System from Pion Scattering}

W. R. Gibbs and B. F. Gibson (Los Alamos)
The ratios of differential cross sections for the clastic scattering of $\pi^{+}$and $\pi^{-}$from the ${ }^{3} \mathrm{H}-{ }^{3} \mathrm{He}$ isodoublet have been reported from two separate experiments, ${ }^{1,2}$ and a third measurement is being analyzed. ${ }^{3}$ Those experiments were motivated as a search for evidence of charge-symmetry breaking (CSB) in the hadronic strong force. Such CSB has apparently been observed in the trinucleon binding energy, because only some $670 \mathrm{keV}$ out of the $760-\mathrm{keV}{ }^{3} \mathrm{H}-{ }^{3} \mathrm{He}$ binding energy difference is accounted for by the repulsive Coulomb interaction acting between the two protons in ${ }^{3} \mathrm{He}$. Indeed, the measured ratios differ substantially from unity, the value one would anticipate if isospin were strictly conserved. $\mathrm{Kim}, \mathrm{Kim}$, and Landaut studied the effect of the Coulonib force in the pionnucleus interaction and showed it to be nonnegligible. That is, the ratios must deviate from unity when the pion-nucleus Coulomb interaction is included in a model calculation. Such Coulomb effects do not cancel in any of the ratio measurements. However, there is a second reason that the measured ratios must differ from unity: the proton and neutron radii (distributions) in ${ }^{3} \mathrm{H}$ and ${ }^{3} \mathrm{He}$ are not identical. In particular, it appears on the basis of our investigation that one can extract the difference between $r_{p}\left({ }^{3} \mathrm{H}\right)$ and $r_{n}\left({ }^{3} \mathrm{He}\right)$ and that between $r_{p}\left({ }^{3} \mathrm{He}\right)$ and $r_{n 1}\left({ }^{3} \mathrm{H}\right)$ with a precision that excecds the uncertainty in the difference between $r_{p}\left({ }^{3} \mathrm{H}\right)$ and $r_{p},\left({ }^{3} \mathrm{He}\right)$ as determined by existing absolute measurements in elastic electron scattering. We find, using standard multiple-scattering analysis techniques, that the difference between the odd radii can be determined with an accuracy of $\pm 0.007 \mathrm{fm}$ and that for the even radii with an accuracy of $\pm 0.010 \mathrm{fm}$ from the existing data.

The structure of the trinucleon bound states is determined by the properties of the underlying nuclear forces. ${ }^{5}$ One understands qualitatively the relative sizes of ${ }^{3} \mathrm{He}$ and ${ }^{3} \mathrm{H}$ from our knowledge of the nucleon-nucleon $(N N)$ interactions. ${ }^{6}$ The $n-p$ force is slightly stronger than the $n-n$ (or $p-p)$ force. The deuteron is bound and the $n-p$ spin-singlet scattering length $(-23.7 \mathrm{fm})$ is larger in magnitude than the $n n$ scattering length $(\simeq-18 \mathrm{fm}$ ), where $a \rightarrow-\infty$ implies a bound state with zero energy binding. Therefore, one expects the radius of the odd nucleon in the trinucleon ground states (proton in ${ }^{3} \mathrm{H}$ or neutron in ${ }^{3} \mathrm{He}$ ) to be smaller than the radius of the like pair of (even) nucleons. It follows that the proton radius of ${ }^{3} \mathrm{H}$ should be smaller than the neutron radius. State-of-the-art Faddeev calculations ${ }^{6}$ employing contemporary nucleon-nucleon force models yield a difference between these two radii of about $0.15 \mathrm{fm}$. In the absence of the Coulomb interaction between the two protons in ${ }^{3} \mathrm{He}$, charge symmetry would require thai the ${ }^{3} \mathrm{H}$ and ${ }^{3} \mathrm{He}$ systems be identical. (We neglect for the moment in this discussion any small charge symmetry breaking in the strong interaction; i.e., the $n-n$ force is assumed to be identical to the strong $p-p$ force.) Including the repulsive Coulomb interaction in the Faddeev calculations for ${ }^{3} \mathrm{He}$ leads to an increase in the proton radius (over the neutron radius in ${ }^{3} \mathrm{H}$ ) estimated to be $0.030 .04 \mathrm{fm}$, based upon separate least square fits to a number of ${ }^{3} \mathrm{H}$ and ${ }^{3} \mathrm{He}$ model calculations." This Coulomb interaction also affects the ${ }^{3} \mathrm{He}$ neutron radius, since the increased separation of the two protons means that the neutron 
is less bound; that is, the neutron distribution is also expanded. The neutron radius is increased (over the proton radius in ${ }^{3} \mathrm{H}$ ) by $0.020 .03 \mathrm{fm}$ in the model Faddeev calculations. Thus, to investigate the Coulomb effect it is natural to compare the two-even or two-odd radii.

The sizes of ${ }^{3} \mathrm{H}$ and ${ }^{3} \mathrm{He}$ have been studied experimentally ${ }^{x-18}$ in numerous clastic-electron-scattering experiments. A recent analysis of the charge-scattering data by the Saclay group ${ }^{19}$ yiclded point proton radii values of $r_{p}\left({ }^{3} \mathrm{Hc}\right)=1.75$ $\pm 0.04 \mathrm{fm}$ and $r_{n}\left({ }^{3} \mathrm{H}\right)=1.61 \pm 0.04 \mathrm{fm}$. Charge radii have also been calculated using ${ }^{3} \mathrm{He}$ and ${ }^{3} \mathrm{H}$ one-body densities generated from wave-function solutions of the Faddeev equations for various contemporary nucleon-nucleon potential models both with and without including two-pion-exchange, three-body-force interactions..$^{5-7}$ Using an interpolating fit to estimate the point proton radii yiclds $^{7}$

$$
\begin{aligned}
r_{\mu}\left({ }^{3} \mathrm{Hc}\right) & =1.77 \mathrm{fm} \\
r_{\mu}\left({ }^{3} \mathrm{H}\right) & =1.58 \mathrm{fm} .
\end{aligned}
$$

The $1.77-\mathrm{fm}$ value for ${ }^{3} \mathrm{He}$ includes an $0.04-\mathrm{fm}$ increase in its size caused by pointCoulomb repulsion between the two protons. The model difference of $0.19 \mathrm{fm}$ is consistent with the results of the Saclay analysis.

What is known about the neutron radii of ${ }^{3} \mathrm{H}$ and ${ }^{3} \mathrm{H}$ ? The spin of the trinucleon is given essentially by that of the odd nucleon. However, it is difficult to extract a neutron radius for ${ }^{3} \mathrm{He}$ from magnetic-clastic-electron scattering, because meson-exchange-current corrections are sizable. ${ }^{21-23}$ In fact, the magnetic properties of ${ }^{3} \mathrm{He}$ are one of the few examples where meson-exchange currents manifest themselves in an unmistakable way. Using only nuclcon one-body currents, one is unable to account for the experimental "He magnetic form factor: irrespective of the $N N$ and three-body-force models employed, the theoretical value for the ${ }^{3} \mathrm{He}$ magnetic moment in impulse approximation (no meson-exchangecurrent correction included) is $20 \%$ smaller in magnitude than the experimental value $(-2.1275 \mathrm{~nm}) .{ }^{24}$ Even if one had full confidence in the available models for calculating the meson-exchange-current corrections to ${ }^{3} \mathrm{He}$ magnetic scattering, the uncertainty in the ${ }^{3} \mathrm{He}$ magnetic radius is a factor of two larger than that attributed to the charge-radius measurement, 25 which would imply that the neutron-proton radius difference in ${ }^{3} \mathrm{He}$ is essentially unknown. The uncertainty in our knowledge of the triton-magnetic radius is even larger. However, that is irrelevant for our purpose, because it is the proton (the odd nucleon) in ${ }^{3} \mathrm{H}$ that carries most of the spin. That is, for ${ }^{3} \mathrm{H}$ it is impossible to extract a neutron radius from electron-scattering data.

For these reasons, one is led to pursue pion scattering to determine the relative radii in the $A=3$ system, where meson-exchange-current contamination is expected to be minimal. Near resonance, the $\pi^{+}-p$ interaction dominates $\pi^{+}$scattering and the $\pi^{-} . n$ interaction dominates $\pi^{-}$scattering. That is, the $\pi$-nucleon coupling is much stronger than the $\pi-\pi$ coupling. Hence, the situation is very different from that in electron scattering where the coupling of the electron to 
the pion is the same as that of the electron to the proton. In fact, we can place experimental limits on the strong-interaction contributions of pion exchange currents in pion scattering. If we look at the double-charge-exchange channel, the calculations ${ }^{26}$ and experiments ${ }^{27}$ limit the contribution of this effect to less than $1 \mu \mathrm{b} / \mathrm{sr}$ at forward angles (and the cross section decreases with angle). Since the smallest cross section to be considered here is the order of $1 \mathrm{mb} / \mathrm{sr}$, it is clear that this contamination is no more than $0.1 \%$ in cross section ( $3 \%$ in amplitude). Assuming that pion multiple-scattering effects can be properly accounted for, ratio measurements should be very sensitive to differences in the odd-nucleon and even-nucleon matter distributions. Similar techniques have been used in electron-scattering ratio measurements to explore the charge distribution differences of isotope and isotone sequences.

Let us consider three ratios of pion-trinucleon clastic scattering cross sections. First, the ratio

$$
r_{1}=\frac{\sigma\left(\pi^{+3} \mathrm{H}\right)}{\sigma\left(\pi^{-3} \mathrm{He}\right)}
$$

involves primarily the pion strong interaction with the odd nucleon in each nucleus. That is, in the region of the $(3,3)$ resonance, $\pi^{+} p$ and $\pi^{-} n$ scattering dominate over $\pi^{-} p$ and $\pi^{+} n$. Clearly the coherent Coulomb scattering does not cancel from the ratio, but away from forward angles, the strong interaction should be much more important. Thus, $r_{i}$ should be sensitive to the ratio of the odd-nucleon form factors--in the single-scattering (impulse) approximation, this is exactly what one would measure if only the dominant $\pi^{+} p$ and $\pi^{-} n$ interactions were retained. Both spin-flip and nonspin-flip scattering from the odd nucleon are important, so that the individual cross sections and $r_{1}$ are sensitive to both processes. Second, the ratio

$$
r_{2}=\frac{\sigma\left(\pi^{-3} \mathrm{H}\right)}{\sigma\left(\pi^{+3} \mathrm{He}\right)}
$$

involves primarily the pion strong interaction with the even nucleons in each nucleus. Again, the Coulomb effects do not cancel in the ratio. However, because the like nucleons are essentially paired in spin (to spin 0), spin-flip scattering is minimal. Thus, ra should be sensitive to the ratio of the even-nucleon form factors and is dominated by nonspin-flip scattering. Finally, the "super ratio"

$$
\begin{aligned}
R & =r_{1} r_{2} \\
& =\frac{\sigma\left(\pi^{+3} \mathrm{H}\right) \sigma\left(\pi^{-3} \mathrm{H}\right)}{\sigma\left(\pi^{-3} \mathrm{He}\right) \sigma\left(\pi^{+3} \mathrm{He}\right)}
\end{aligned}
$$

should be least sensitive to model uncertainties in the pion-nucleus scattering theory (as well as experimental normalizations). While the Coulomb interaction does not cancel, the calculation of $R$ is less sensitive to any model dependence in those effects than are the individual ratios $r_{1}$ and $r_{2}$. Because the ${ }^{3} \mathrm{He}$ nucleus is expected to be larger than that of ${ }^{3} \mathrm{H}$, so that its form factor falls faster, we 
anticipate (in general) that $R>1$. Similar conclusions can be reached for $r_{1}$ and $r_{2}$, although they are subject to greater uncertainty.

In our investigation we have treated the even and odd radii (actually their difference, as discussed below) as variables by rescaling the individual proton and neutron densities obtained from Faddeev equation calculations. Thus, we implicitly assume a shape for the densities given by theory. We have used two different densities as starting points: ${ }^{25}$ one from the solution including both two- and three-body forces (the "standard" casc) and one using two-body forces alone (denoted by a subscript " 2 " on the letter identifying the case). Since the momentum transfer probed is much smaller than that at which the first zero in the form factor occurs, the basic shape of the curve is primarily determined by the radius. The sensitivity to this assumption is tested by comparing results with the two forms just mentioned.

While there is, unavoidably, a degree of uncertainty because of the manner in which the strong scattering process is treated (and its effect on the Coulomb interference), we have found that the sensitivity to changes in the three-nucleon form factors, at a certain scale of variation of the radii, is considerably greater than this uncertainty. In order to determine the uncertainties due to the approximations in our scatterii.g theory, we have varied the parameters controlling its structure within what we believe to be reasonable bounds, keeping in mind that it should provide an acceptable representation of the differential cross sections. We note that the errors quoted for these (latter) measured cross sections are substantially larger than those of the measured ratios. While we find that there is relatively little sensitivity of " $R$ " to the scattering theory, for the ratios $r$, and $r_{2}$ this is not true. We discuss this point in more detail below. In the future, we may anticipate that the scattering calculation can be made with greater certainty using multiple scattering techniques, ${ }^{2 ! 9}$ but for the present investigation, we have simply varied the relevant controlling parameters. The different cases are defined in Table I with a letter denoting each. For each case, a $\lambda^{2}$ comparison with the recent LAMPF data of Nefkens et al. ${ }^{2}$ and of Pillai et al. ${ }^{2}$ for the observable " $R$ " was constructed as a function of the even $\left(r_{t}\right)$ and odd $\left(r_{0}\right)$ neutron radii. The corresponding even $\left({ }^{3} \mathrm{He}\right)$ and odd $\left({ }^{3} \mathrm{H}\right)$ proton radii were held fixed at values within the errors quoted from the electron-scattering determination. This $x^{2}$ function was found to be well represented by a parabolic form in two dimensions:

$$
\begin{aligned}
\lambda^{2}= & {\left[\left(r_{t}-r_{t}\right) \cos (0)+\left(r_{0}-r_{0}^{c}\right) \sin (0)\right] / a^{2} } \\
& +\left[-\left(r_{t}-r_{\epsilon}^{c}\right) \sin (0)+\left(r_{0}-r_{o}^{c}\right) \cos (0)\right] / b^{2}+\chi_{\min }^{2} .
\end{aligned}
$$

The quantities $r, r, 0, a, b$, and 12 , which characterize cach fit, are quoted in the second half of Table l. They were obtained by fitting this function to

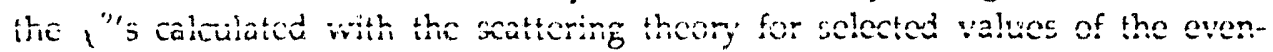
and odd-neutron radii. It is estimated that the uncertainty in the values, or $r_{\epsilon}^{r}$ and $r_{o}^{c}$ extracted in this second fitting procedure, is $\pm 0.001 \mathrm{fm}$. We observe 


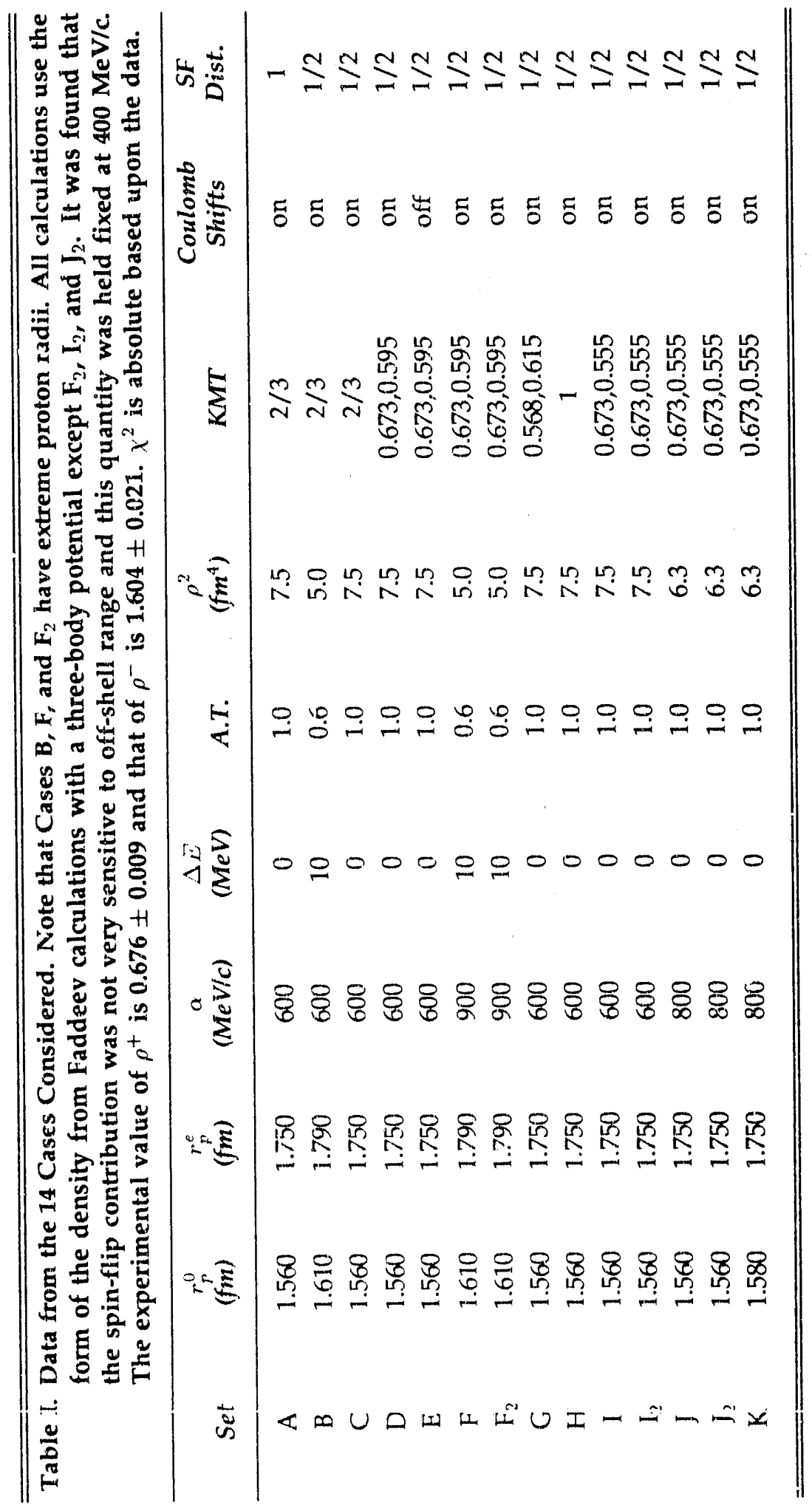




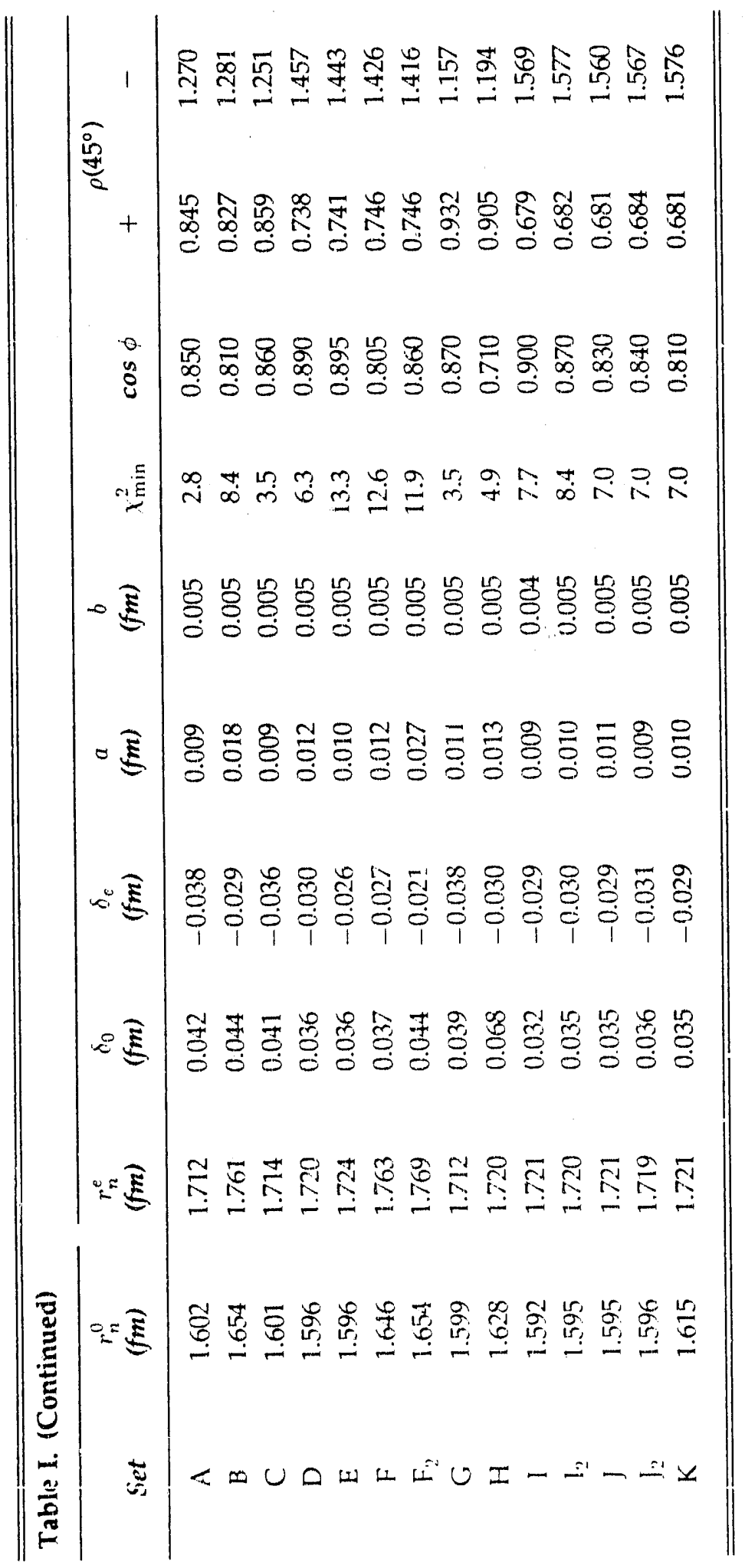


that only the differences between the two-odd and two-even radii are relevant for the fits. While Table I lists results for several different radii, a comparison of Cases $\mathrm{J}$ and $\mathrm{K}$ shows the effect of this change alone. This character of the fits is essential to the analysis since the errors on the proton radii are of the same order as the effect that we are investigating.

The underlying scattering theory is based on a nonlocal optical potential that has frequently been used in investigations of pion scattering from heavier nuclei. ${ }^{29-31}$ The spin-flip cross section (incoherent with the nonspin-flip cross section) is calculated in the distorted-wave-impulse approximation by using the wave function resulting from the scattering from the two like nucleons. The logic of KMT (discussed in sol e detail below) would imply that there should be a factor of $1 / 2$ multiplying the potential used in this scattering to obtain the $t$-matrix. For most of our distorted-wave calculations we have used this factor; it is, however, not very important for the analysis pertaining to $R$, as can be seen by comparing Cases $\mathrm{A}$ and $\mathrm{C}$ in Table I where this variation is the only difference.

Our scattering theory has been described in detail in Refs. 29-31. The primary uncertainty in our analysis is related to the treatment of the following principal features:

1. Off-shell ranges. The $\pi-N$ scattering amplitudes contain $s$-wave and $p$ wave ranges, which can be varied independently. While the off-shell dependence of the 1 -matrix can be determined from a model of the strong interaction in principle, in practice the value obtained depends on the model used and/or on the means employed to determine it from the extant experimental data. ${ }^{32}$ There is also the uncertainty arising because the value within the nucleus may be modified by the lifetime of the delta resonance, adding further nonlocality to the interaction. We found that the calculated ratios depended only weakly on these variables (on a scale set by changes in the radius differences), although the absolute cross section approximately scales with these off-shell ranges. Because of the lack of sensitivity in the ratios to these quantities, we set the $s$-and $p$-wave off-shell ranges equal to one another.

Since the spin-dependent contribution to the scattering (all of the spin was assumed to be carried by the odd nucleon) was treated by a distorted-waveimpulse approximation, in which the distorting wave was assumed to be caused by scattering from the even nucleons, it was necessary to construct a transition operator based on the measured phase shifts. Since this transition operator was treated as nonlocal, there is also an off-shell range associated with it. The dependence of the ratios on this spin-flip off-shell range was even smaller than that due to the nonspin-flip range, so we fixed its value (at $400 \mathrm{McV} / \mathrm{c}$ ).

2. Effective energy. Because of the restriction on the allowed intermediate states in the nuclear medium, the $t$-matrix used in scattering from a bound nucleon differs from that found in free space scattering. ${ }^{33,34}$ The effective energy shifts, as explained in Ref. 29, can be treated as a function of the pion-nucleus 
angular momentum. Since such a calculation is beyond the scope of the present work, we used a single number for the energy shift ${ }^{35}$ (in practice usually zero).

3. "Angle transform." Because of recoil of the nucleon in the nuclear medium, the effective $s$ - and $p$-wave amplitudes are modified. ${ }^{36,29}$ We used a rather standard prescription ${ }^{37}$ but considered the possibility of an additional multiplying factor in this correction. A calculation of the type reported in Ref. 29 would provide a more complete description of the recoil effects, but a relativistic version would be needed for these energies.

4. KMT factor. It is clear that a given nucleon cannot be struck successively by the projectile in a multiple-scattering series based on a $t$-matrix. Kerman, McManus, and Thaler pointed out ${ }^{38}$ that the geometric multiple-scattering series can be summed under this restriction by using, for the potential, $(A-1) / A$ times the impulse approximation for scattering and then multiplying the scattering amplitude resulting from the solution of the Schrödinger equation by the inverse of this $(A-1) / A$ factor. For large $A$ this is a small correction, but for the threebody system, it is very important. The calculation " $\mathrm{H}^{\prime}$ in Table I shows the effect of ignoring this correction entirely. The values of $\rho^{+}=\sigma\left(\pi^{+3} \mathrm{H}\right) / \sigma\left(\pi^{+3} \mathrm{He}\right)$ and $\rho^{-}=\sigma\left(\pi^{-3} \mathrm{H}\right) / \sigma\left(\pi^{-3} \mathrm{He}\right.$ ) at $45^{\circ}$ (where the predictions of the theory should be the most reliable and the dependence on the radii the least) are in poor agreement with the data when the KMT factor is omitted.

To understand the physics of this factor, consider it to be a variable "g." Start with the (single-scattering) impulse approximation for the elastic scattering

$$
\left[f\left(\vec{k}, \vec{k}^{\prime}\right) S\left(\left|\vec{k}-\vec{k}^{\prime}\right|\right)\right],
$$

multiplied by $!$, as the potential in the Schrödinger equation and then divide the resulting amplitude by $g$. If $g$ is very small, then the single scattering impulse approximation is recovered. If the interaction is very strong (and $g \approx 1$ ), then the nucleus appears to be nearly "black," and the cross section will be approximately independent of the strength of the potential, resulting in values for $\rho^{+}$and $\rho^{-}$ nearly equal to one. If the interaction is weak (or if $g$ is small), then the cross sections will scale as the square of the strength of the potential. Thus, the factor $g$ can be thought of as a variable that interpolates between these two extremes.

Consider the cross sections at $45^{\circ}$ where the spin-flip contribution is small. If we postulate two "strong" interactions of magnitude 3 and one "weak" interaction of magnitude 1 (which is the approximate ratio of the $\pi^{+} p$ to $\pi^{-} p p$-wave amplitudes), then the cross section ratio for the single-scattering-impulse approximation is $(3+1+1)^{2} /(3+3+1)^{2}=0.51$ for $\rho^{+}$and the inverse 1.96 for $\rho^{-}$. The experimentally observed ratios are 0.676 for $\rho^{+}$and 1.604 for $\rho^{-}$. A potential with $g=1$ gives 0.90 for $\rho^{+}$and 1.18 for $\rho^{-}$(approaching the "black" limit), while $g=2 / 3$ gives 0.85 and 1.27 (somewhat closer to the experimental result). As $g$ is reduced, the phase of the strong amplitude is rotated, affecting the interference with the Rutherford amplitude as well. In fact, $g<1$ reduces this Coulumb correcion for boili $r_{1}$ and $r .2$. Whithe the ratio $R$ is much less affected, 
this dependence on $g$ is one of the primary sources of uncertainty in the present analysis.

The situation becomes even more complex when the interactions of the projectile with the target protons and nucleons are allowed to differ. In Table l, we include calculations for two such prescriptions; compare $C$ and $D$ with $E$. The values of $g$ were calculated using the ratio of $p$-wave strengths for the optical model. (While this ratio is complex, the imaginary part is very small.) The D prescription is preferred because it gives a better representation of $\rho^{+}$and $\rho^{-}$. In fact, by reducing the "strong" scattering KMT factor to $g=0.555$, one obtains the most satisfactory agreement. Therefore, several cases $(I \rightarrow K)$ are illustrated with this value.

5. True absorption. A purely imaginary constant multiplying the square of the density was used to provide a potential representing the effect of pion absorption. ${ }^{39}$ The coefficients used are in rough agreement with values determined from other nuclei. ${ }^{40}$ [The units used in Table I for this factor are such as to yield $\mathrm{fm}_{i}{ }^{-2}$ when it multiplies the square of the nucleon density (in nucleons/ $\left.\mathrm{fm}^{3}\right)$.] Scaling from experience with calcium and carbon, assuming a proportionality to the number of $n-p$ pairs, $N Z$, would lead one to expect a number around $12 \mathrm{fm}^{4}$. Variation from this value was allowed in order to obtain a satisfactory representation of the differential cross sections.

6. Coulomb energy shifts. Since the pion will gain or lose energy approaching the nucleus, depending on the sign of its charge, the effective energy for the strong interaction will differ for $\pi^{+}$and $\pi^{-} .{ }^{41}$ The effect is not very large, but it is of opposite sign for the numerator and denominator in $r_{1}$ and $r_{2}$, so that it is important for those ratios. It is much less important for the ratio $R$ (compare Cases $D$ and $E$ in Table I) since the increase in one factor of the numerator or denominator is compensated by a decrease in the other. The values used for the Coulomb energy shifts are:

$$
\begin{array}{ll}
\pi^{+} \mathrm{T} & -0.9 \mathrm{MeV} \\
\pi^{+} \mathrm{He} & -1.8 \mathrm{MeV} \\
\pi^{-\mathrm{T}} & +0.9 \mathrm{MeV} \\
\pi^{--\mathrm{He}} & +1.8 \mathrm{MeV}
\end{array}
$$

\section{Super Ratio $R$}

We have discussed the various assumptions that went into the calculations summarized in Table I. We now deal with the results shown in the second part of the table. The values of $\delta_{e}=r_{e}^{n}-r_{e}^{p}$ and $\delta_{o}=r_{0}^{n}-r_{o}^{p}$ are seen to closely cluster

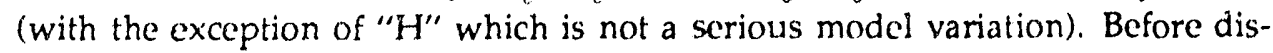
cussing this uncertainty, we note that the elastic scattering angular distributions are well represented as shown in Fig. 1 . The minimum in each curve is caused by the $p$-wave nature of pion-nucleon scattering and is unrelated to the minimum in the three-body form factor. In Figs. 2 and 3, we illustrate the effect on the 


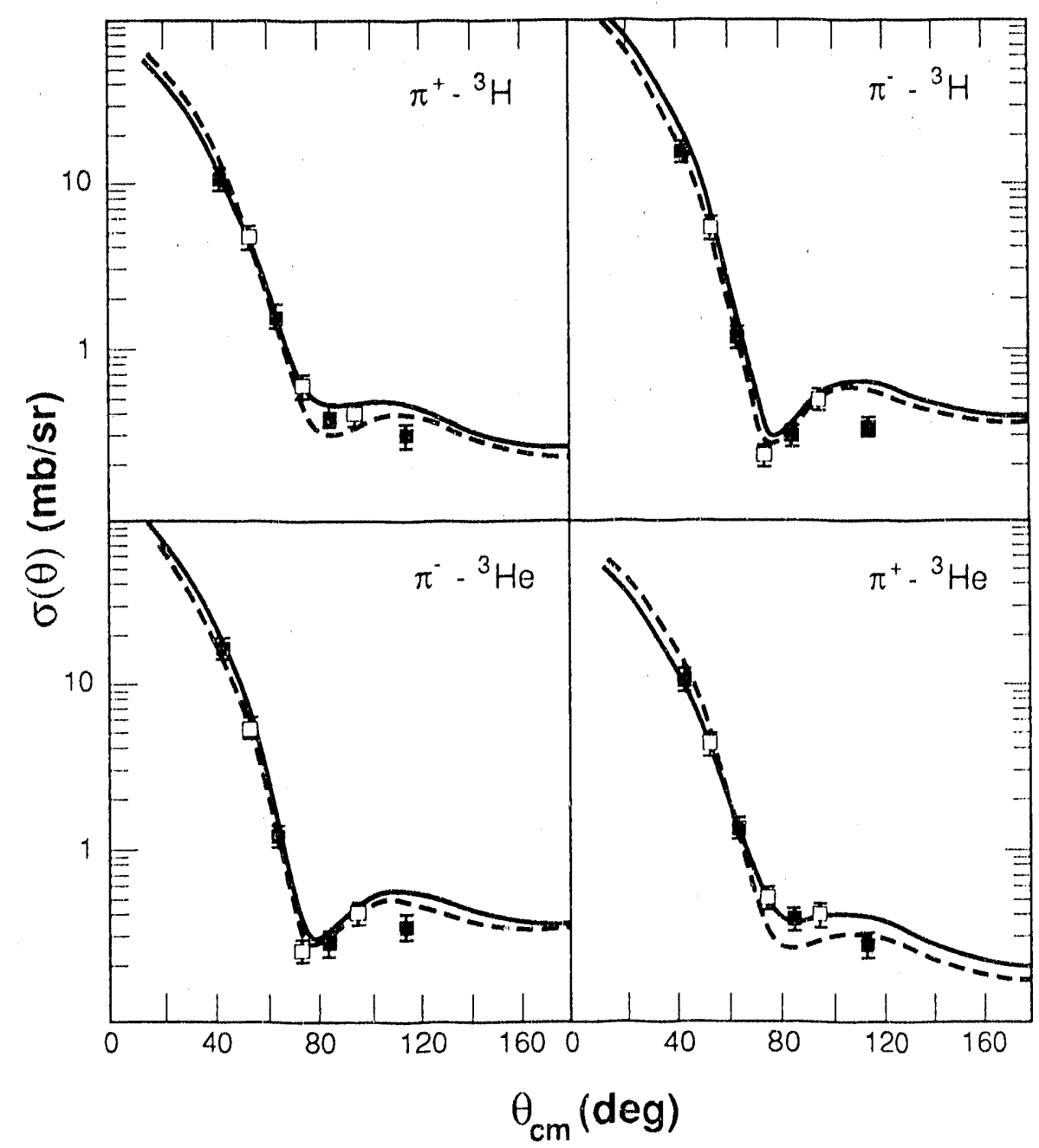

Fig. 1. Angular distributions for the four combinations. The agreement with the data is moderate. The solid curves correspond to Case I and the dotted to Case $A$. While there is not much to distinguish the two to the eye, Case I gives a much better representation of $p^{+}$and $p^{-}$at $45^{\circ}$. 


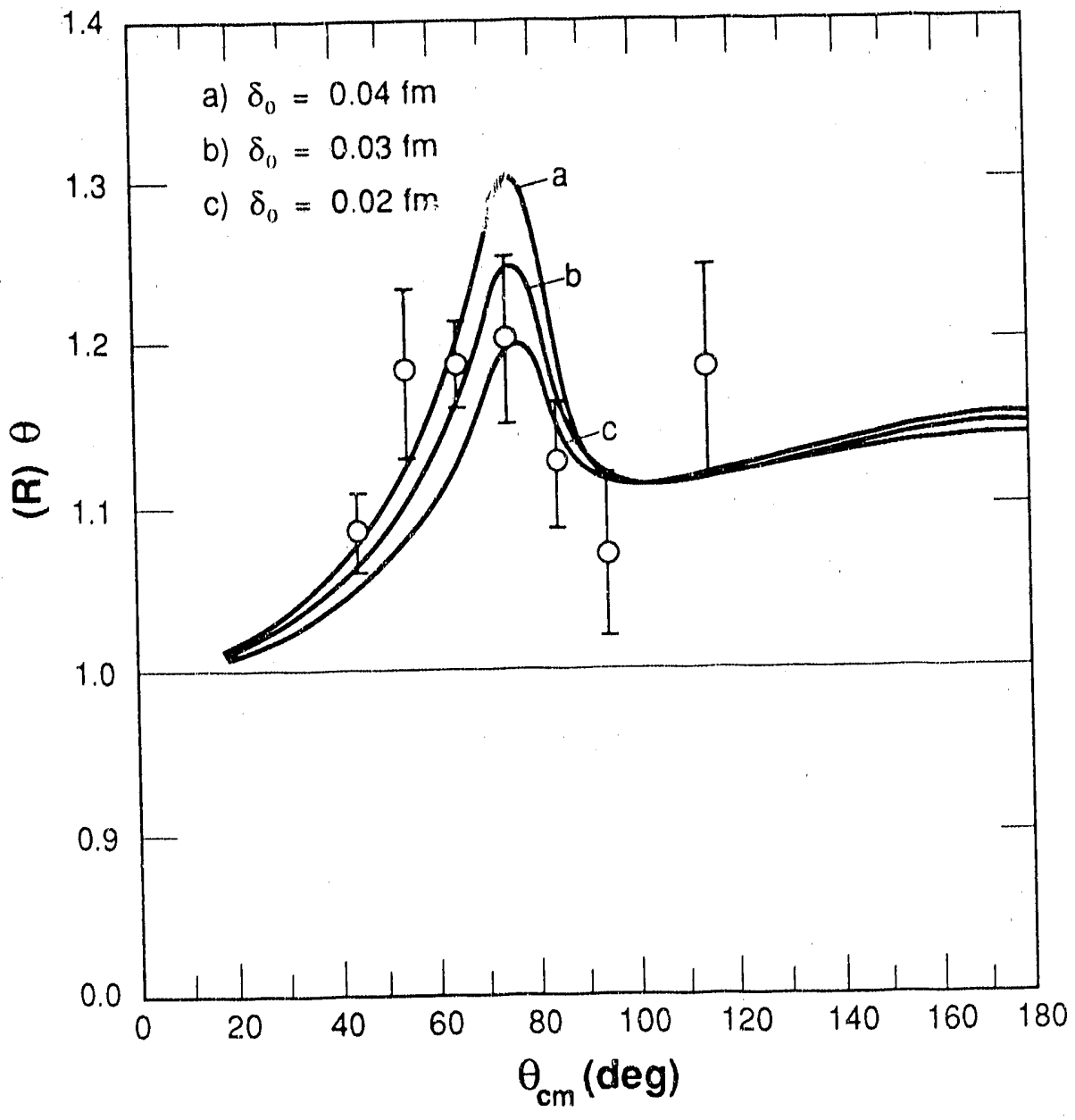

Fig. 2. Example variations with respect to the odd-neutron radius of the calculated " $R$ " compared with the data. It is clear that the minimum region (where the "spin-flip" term, depending only on the odd-nucleon form factor, dominates) controls the fit to the data. The curves are calculated for $r_{n}^{e}$ fixed at $1.720 \mathrm{fm}$ and $r_{n}^{\circ}$ taking values of $1.58,1.59$, and $1.60 \mathrm{fm}$ for Case $A$. 


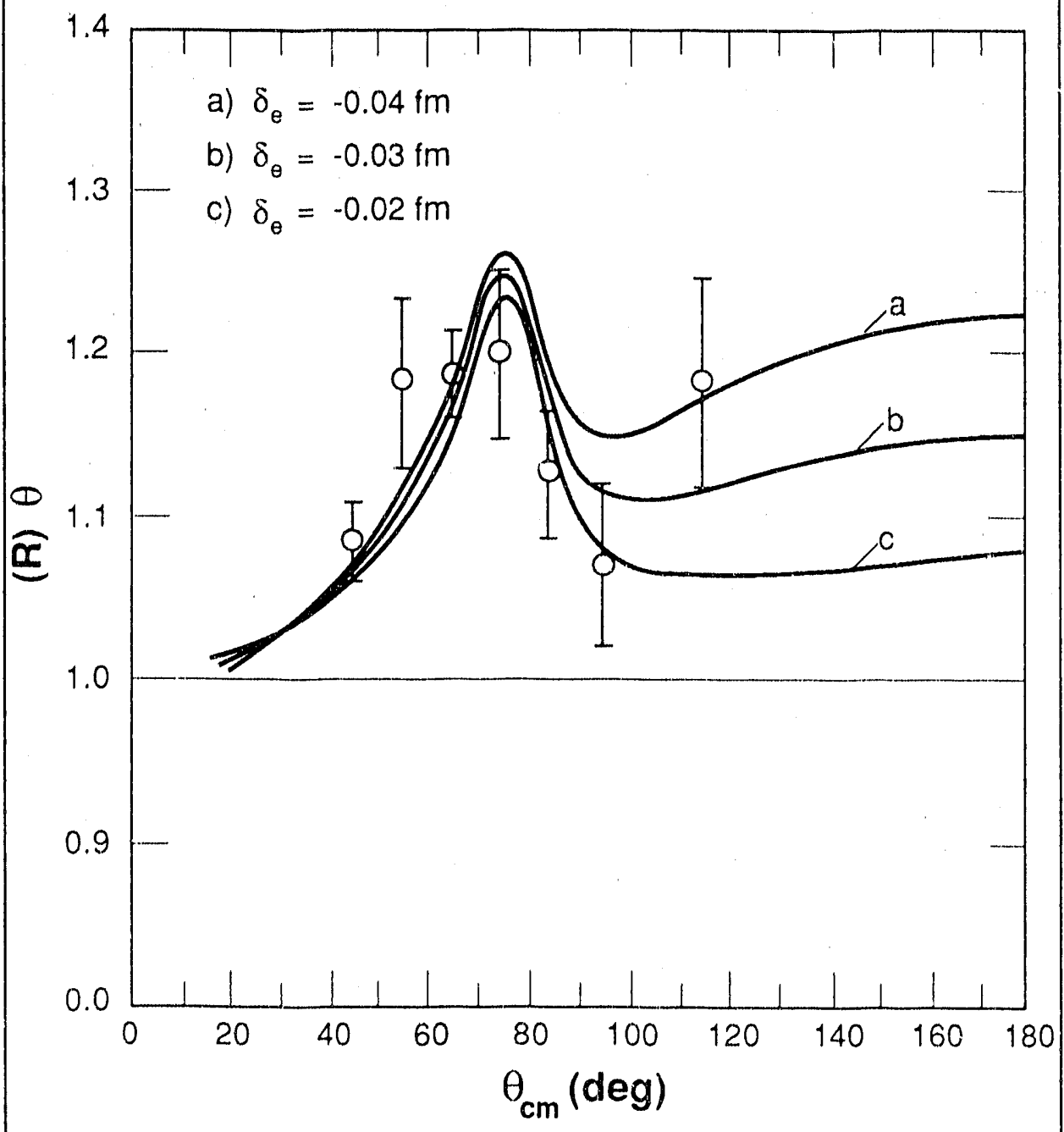

Fig. 3. Example variations with respect to the even-neutron radius of the calculated " $R$ " compared with the data. Here the entire angular range (but especially the part at large angles) is important. Clearly, high-quality backangle data would reduce the allowed variations in this variable. The curves are calculated for $r_{n}^{0}$ fixed at $1.59 \mathrm{fm}$ and $r_{n}^{e}$ taking on values of 1.71, 1.72, and $1.73 \mathrm{fm}$ for Case $A$. 
predictions for $R$ (compared with the data) attributable to variations in the radii of $\pm 0.01 \mathrm{fm}$ about a value near the best fit. It is clear that the odd-radius difference is determined by the data around the minimum -where the spin flip, coming from the odd nucleon, dominates--whereas the even-radius difference is determined by the data at many angles.

The results of our investigation as summarized in Table I are shown in graphical form in Fig. 4. The curves are computed using the average of all of the $\chi^{2}$ surfaces and represent the loci of all points $\chi^{2}-\chi_{\min }^{2}=1$ and 4 . TH.e point corresponding to no effect [i.c., $r_{o}\left({ }^{3} \mathrm{He}\right) \equiv r_{o}\left({ }^{3} \mathrm{H}\right)$, ctc.] is the upper lefthand corner of the graph at zero. It is clear that there is definite evidence for a difference in the two radii and that a value can be extracted with a specified error. To estimate the crror in our determination of $\delta_{c}$ and $\delta_{o}$, a conservative approach would be to compute the average and variance of all of the central values and to convolute the error obtained with the average statistical error determined from the ellipses. Tt: s procedure gives:

$$
\delta_{e}=-0.030 \pm 0.010 \mathrm{fm}
$$

(of which $0.005 \mathrm{fm}$ of the crror is theoretical), and

$$
\delta_{o}=0.038 \pm 0.007 \mathrm{fm}
$$

(of which $0.004 \mathrm{fm}$ of the error is theoretical).

A more optimistic approach in estimating the error would entail the elimination of those cases that do not give a good fit to $\rho^{+}$and $\rho^{-}(A, B, C$, and $G)$ and those cases that have a large $\chi^{2}\left(E, F\right.$, and $\left.F_{2}\right)$. This leaves Cases $D, I, I_{2}, J$, $\mathrm{J}_{2}$, and $\mathrm{K}$. An average of these cases alone yields

$$
\delta_{e}=-0.030 \pm 0.008 \mathrm{fm}
$$

(of which $0.001 \mathrm{fm}$ of the error is theoretical), and

$$
\delta_{0}=0.035 \pm 0.007 \mathrm{fm}
$$

(of which $0.001 \mathrm{fm}$ of the error is theoretical).

For purposes of comparison with these radii differences extracted from our analysis of pion $-{ }^{3} \mathrm{H} /{ }^{3} \mathrm{He}$ scattering, we have constructed three separate trinucleon models in which a single parameter was adjusted to yield the experimental ${ }^{3} \mathrm{H}$ and ${ }^{3} \mathrm{He}$ binding energies:

1. We combined the "stiff" Reid-soft-core (RSC) $N N$ potential42 with the Tucson-Melbourne (TM) two-pion-exchange, three-nucleon force. ${ }^{43}$ We chose the pion-nucleon form factor range $\Lambda$ in the TM 3BF such that the triton binding energy was $8.48 \mathrm{MeV}$. Turning on a point Coulomb interaction between the two protons in ${ }^{3} \mathrm{He}$ then yielded a binding energy of $7.81 \mathrm{McV}$. To reduce this to the experimental $7.72 \mathrm{MeV}, \Lambda$ was reduced by $1.5 \%$. 


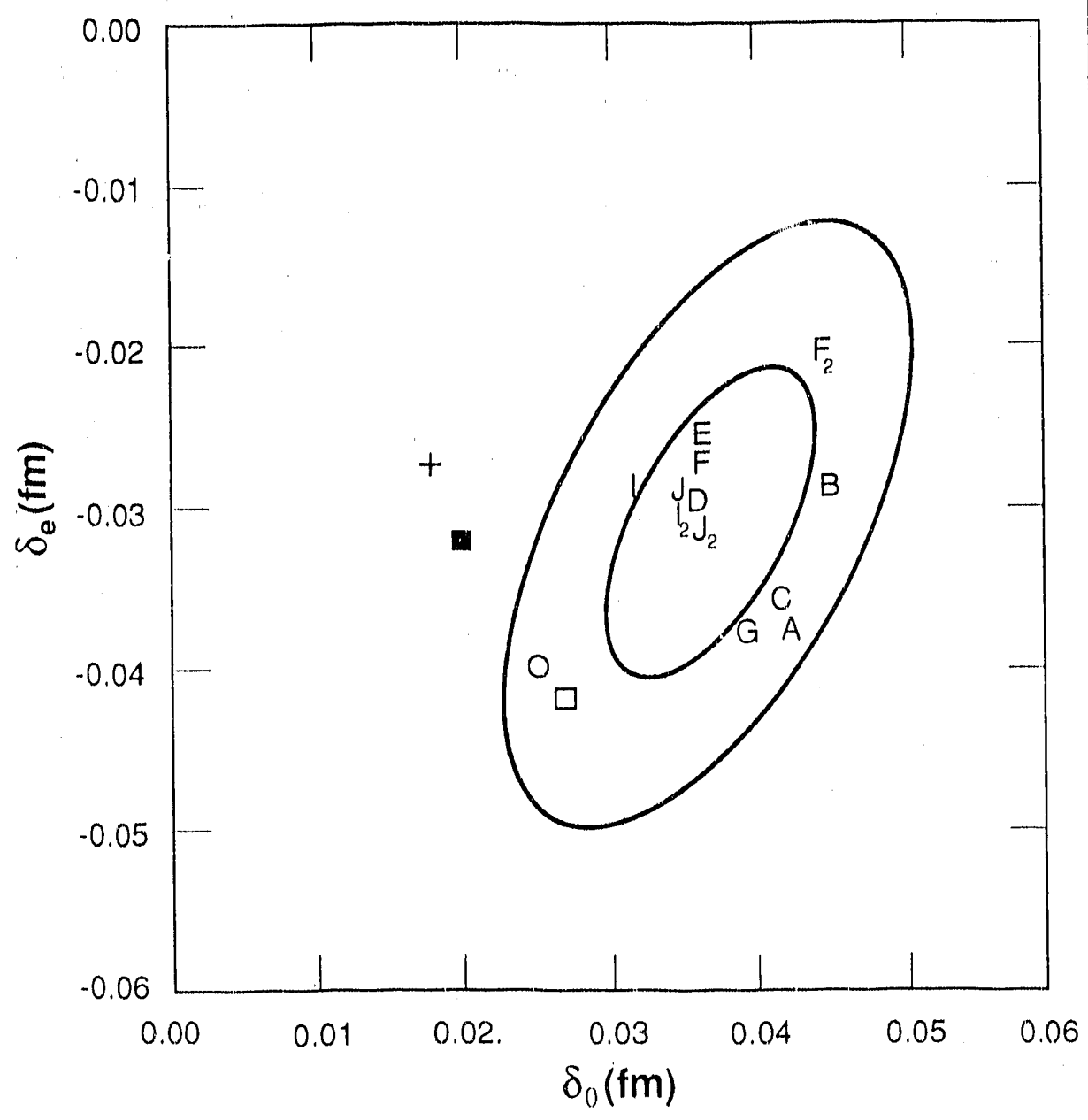

Fig. 4. Loci of the $x^{2}$ surfaces $x^{2}=x_{\min }^{2}+1$ and +4 . The calculated $x^{2}$ is the average of all of the cases shown; hence, the ellipse is spread by the distribution of centroids. Note that Case $F_{2}$ is not as aberrant as it might seem since its position along the major axis is ill deterrined. Case $H$ is not shown and the point $K$ is degenerate with $I$. 
2. An arralogous procedure was followed using the Argonne $V_{14}$ (AV14) $N N$ potential ${ }^{14}$ combined with the Brazilian (BR) three-body force model. ${ }^{45}$

3. Both of these three-body force models are isoscalar. Therefore, we considered a third model in which the $\ell=0$ spin-singlet central potential strength was increased to obtain the desired $8.48-\mathrm{McV}$ binding of the triton in the AV14 $N N$ force model. (The original AV14 model yields a binding energy for the triton of $7.67 \mathrm{MeV}$ in a 34-channel calculation.) The corresponding ${ }^{3} \mathrm{He}$ binding erergy was again $7.81 \mathrm{MeV} ; 7.72 \mathrm{MeV}$ was attained when the spin-singlet enhancement factor was decreased by $0.6 \%$.

In each of these three cases, one finds $\delta_{o}=0.020 \mathrm{fm}$ and $\delta_{e}=-0.032 \mathrm{fm}$, when the Coulomb interaction between the two protons is the only charge symmetry breaking interaction considered; $\mathrm{BE}\left({ }^{3} \mathrm{H}\right)=8.48 \mathrm{MeV}$ and $\mathrm{BE}\left({ }^{3} \mathrm{He}\right)=7.81 \mathrm{MeV}$. This is indicated by the black square in Figs. 4 and 5 . When the models were adjusted (as described) to yicld the experimental $\mathrm{BE}\left({ }^{3} \mathrm{He}\right)=7.72 \mathrm{McV}$, then in each of the first two models, one finds $\delta_{o}=0.027 \mathrm{fm}$ and $\delta_{e}=-0.042 \mathrm{fm}$, whereas in the pure $N N$ force model, one finds $\delta_{0}=0.025 \mathrm{fm}$ and $\delta_{t}=-0.040 \mathrm{fm}$. That is, charge-symmetry breaking introduced through the $3 \mathrm{BF}$ produces a slightly larger radius effect. This is represented as open symbols in Figs. 4 and 5.

\section{The Ratios $r_{1}$ and $r_{2}$}

While we have observed that the fit to the ratio $R$ is rather stable, such is not the case for the separate ratios $r_{1}$ and $r_{2}$. They are particularly sensitive to the factor $g$. They are also somewhat sensitive to the Coulomb energy shifts. In most instances, the minimum in $r_{1}$ lies at smaller (absolute) values of the two radius differences than for $R$ and the minimum for $r_{2}$ at larger values than for $R$. However, for Case $\mathrm{E}$ (no Coulomb shifts), all three differences are in essential agreement although $\chi^{2}$ from $R$ is large. We note that the prescription that we have used for estimating the shifts gives a maximum effect and the magnitude of the shift probably does need to be reduced.

The conclusions drawn from these two ratios cannot be pushed too far because of the experimental problem of flux normalization. The measurements were made relative to deuterium, and the quoted results are only the true ratios if $\pi^{+}$and $\pi^{-}$scattering from the deuteron are equal. From recent measurements, ${ }^{40}$ one can estimate that this ratio is about $1.02 \pm 0.02$. The value chosen for this correction impacts, to some extent, any conclusions drawn from $r_{1}$ and $r_{2}$. Nevertheless, the conclusions do provide a school for investigating multiple scattering and do indicate which uncertainties are likely to be important for other nuclei where the double-ratio measurement is not possible. 


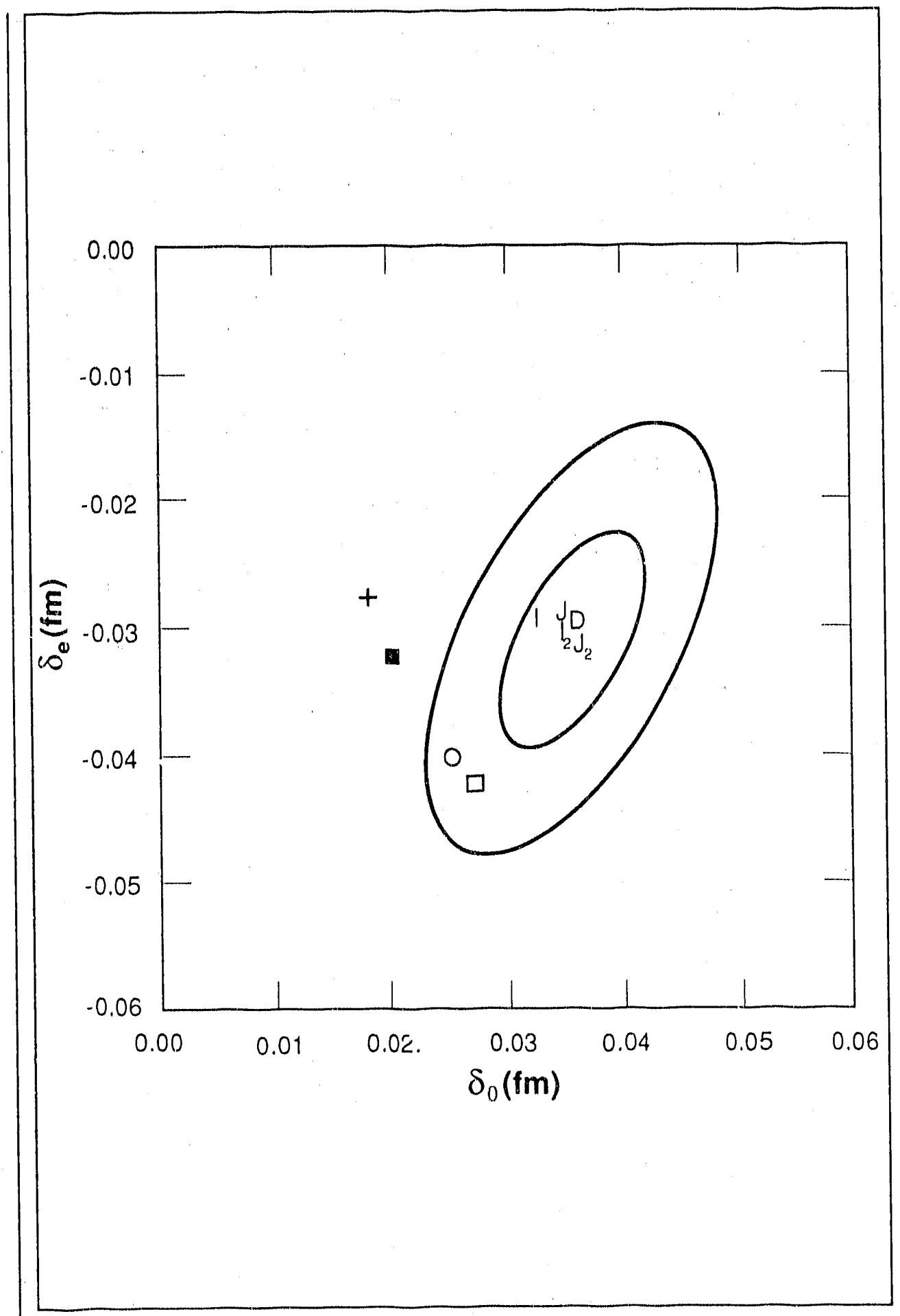

Fig. 5. Same as Fig. 4, except that it includes the reduced, "optimistic" set only. 


\section{Summary}

In summary, calculations for the trinucleon system have established that there is a Coulomb anomaly in terms of the binding energy as is the case for a number of other nuclei. The difference in binding between ${ }^{3} \mathrm{He}$ and the triton is $760 \mathrm{keV}$. Theoretical estimates yield only some $670 \mathrm{keV}$ because of Coulomb repulsion between the two protons. The remaining $90 \mathrm{keV}$ must be attributed to some other charge-symmetry breaking effect. It is natural to ask if an analogous effect exists in the radii. The present treatment lends itself well to answering this question. What we have extracted is the difference in "odd size" $\left(\delta_{o}=0.030 \pm\right.$ $0.008 \mathrm{fm})$ and "even size" $\left(\delta_{e}=-0.035 \pm 0.007 \mathrm{fm}\right)$ between ${ }^{3} \mathrm{He}$ and the triton. Model Faddeev calculations imply differences of the order of $-0.042 \mathrm{fm}$ for the even radii and $0.027 \mathrm{fm}$ for the odd radii. From Fig. 4 or 5 we see, because of the form of the correlated errors, that the general distance of the effect from zero is better determined than either of the individual radius differences. The present analysis indicates that a significant charge-symmetry breaking effect (beyond the Coulomb interaction) has been observed in the differences of the ${ }^{3} \mathrm{H} /{ }^{3} \mathrm{He}$ radii.

\section{References}

1. B. M. K. Nefkens, W. J. Briscoe, A. D. Eichon, D. H. Fitzerald, J. A. Holt, A. A. Mokhtari, J. A. Wightman, M. E. Sadler, R. L. Boudrie, and C. '.. Morris, Phys. Rev. Lett. 52, 738 (1984).

2. C. Pillai, D. B. Barlow, B. L. Berman, W. J. Briscoc, A. Mukhtari, B. M. K. Nefkens, A. M. Petrov, and M. E. Sadler, Phys. Lett. 207B, 389 (1988); C. Pillai, D. B. Barlow, R. S. Kessler, G. J. Kim, B. M. K. Nefkens, J. W. Price, J. A. Wightman, B. L. Berman, W. J. Briscoe, A. Mokhtari, A. M. Petrov, and M. E. Saddler [submitted to Physical Review C (1990)].

3. S. Matthews, private communication, Los Alamos National Laboratory, Los Alamos, New Mexico (1989).

4. K. Y. Kim, Y. E. Kim, and R. H. Landau, Phys. Rev. C 36, 2155 (1987); Y. E. Kim, M. Krell, and L. Tiator, Phys. Lett. 172B, 287 (1986); Y. E. Kim, Phys. Rev. Lett. 53, 1508 (1984).

5. For a discussion of convergence in the calculation of trinucleon bound-state observables as a function of the number of two-body partial waves (for various contemporary $N N$ potential models), see C. R. Chen, G. L. Payne, J. L. Friar, and B. F. Gibson, Phys. Rev. C 31, 2266 (1985). For details of calculations including three-body forces, see C. R. Chen, G. L. Payne, J. L. Friar, and B. F. Gibson, Phys. Rev. C 33, 1740 (1986) and T. Sasakawa and T. Ishikawa, Few Body Systems 1, 3 (1986).

6. J. L. Friar, B. F. Gibson, C. R. Chen, and G. L. Payne, Phys. Lett. 161B, 241 (1985). 
7. J. L. Friar, B. F. Gibson, and G. L. Payne, Phys. Rev. C35, 1502 (1987).

8. P. Dunn, S. B. Kowalski, F. N. Rad, C. P. Sargent, W. E. Turchinetz, R. Goli. kie, and D. P. Saylor, Phys. Rev. C 27, 71 (1983).

9. C. R. Ottermann, G. Köbschall, K. Maurer, K. Röhrich, Ch. Schmitt, and V. H. Walther, Nucl. Phys. A 436, 688 (1985).

10. J. S. McCarthy, I. Sick, and R. R. Whitney, Phys. Rev. C15, 1396 (1977).

11. Z. M. Szalata, J. M. Finn, J. Flanz, F. J. Kline, G. A. Peterson, J. W. Lightbody, X. K. Maruyama, and S. Penner, Phys. Rev. C15, 1200 (1977).

12. G. Retzlaff and D. M. Skopik, Phys. Rev. C29, 1194 (1984).

13. H. Collard, R. Hofstadter, E. B. Hughes, A. Johansson, M. R. Yearian, R. B. Day, and R. T. Wagner, Phys. Rev. 138, B57 (1965).

14. M. Bernheim, D. Blum, W. McGill, R. Riskalla, C. Trail, and T. Stovall, Lett. Nuovo Cimento 5, 431 (1972).

15. R. Arnold, B. T. Chertok, S. Rock, W. P. Schütz, Z. M. Szalata, D. Day, J. S. McCarthy, F. Martin, B. A. Mecking, I. Sick, and G. Tamas, Phys. Rev. Lett. 40, 1429 (1978).

16. J. M. Cavedon, B. Frois, D. Goutte, H. Huct, Ph. Leconte, J. Martino, X.-H. Phan, S. K. Platchkov, S. E. Williamson, W. Boeglin, I. Sick, P. de WittHuberts, L. S. Cardman, and C. N. Papanicolas, Phys. Rev. Lett. 49, 986 (1982).

17. D. H. Beck, S. B. Kowalski, M. E. Schulze, W. E. Turchinetz, J. W. Lightbody, X. K. Maruyama, W. J. Stapor, H. S. Caplan, G. A. Retzlaff, D. M. Skopik, and R. Goloskie, Phys. Rev. 30, 1403 (1984).

18. F. P. Juster, S. Auffert, J.-M. Cavedon, J.-C. Clemens, B. Frois, D. Goutte, H. Huct, P. Leconte, J. Martino, Y'. Mizuno, X.-H. Phan, S. Platchkov, and S. E. Williamson, Phys. Rev. Lett. 55, 2261 (1985).

19. S. Platchkov and B. Frois, private communication, Los Alamos National Laboratory, Los Alamos, New Mexico (1989).

20. W. M. Kloet and J. A. Tjon, Phys. Lett. 61B 356 (1976).

21. M. A. Maize and Y. E. Kim, Nucl. Phys. A4: i, 507 (1983).

22. E. Hadịimichael, B. Coulard, and R. Borr : "'ys. Rev. C27, 831 (1983).

23. W. Str. sve, Ch. Hajduk, and P. U. Sauer, Nucl. Phys. A 405, 620 (1983).

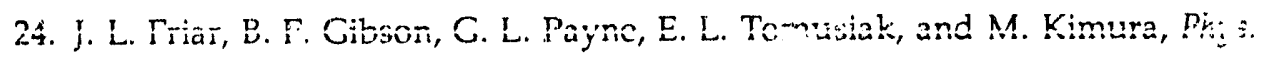
Rev. C 37, 2852 (1988). 
25. J. Martino in The Three-Body Force in the Three-Nucleon System, B. L. Berman and B. F. Gibson, Eds. (Springer-Verlag, Berlin, Heidelberg, New 'ork, London, Paris, Tokyo, 1986), p. 129.

26. N. Aucrbach, W. R. Gibbs, Josepk. N. Ginocchio, and W. B. Kaufmann, Phys. Ren. C 38, 1<.7 (1988); E. Oset, D. Strottman, M. J. Vicente-Vacas, and M. Wei-Hsing, Nucl. Phys. A 408, 461 (1983); J.-F. Germond and C. Wilkin, Lett. Nuovo Cimento 13, 605 (1975).

27. Z. Weinfeld, E. Piasetzky, H. Bacr, R. L. Burman, M. J. Leitch, C. L. Morris et al., Phys. Rev. C 37, 902 (1988).

28. J. L. Friar, B. F. Gibson, G. L. Payne, and C. R. Chen, Phys. Rev. C 34, 1463 (1986).

29. W. B. Kaufmann and W'. R. Gibbs, Phys. Rev. C 28, 1286 (1983).

30. L. S. Kisslinger, Phys. Rev. 98, 761 (1955); J. Revai, Nucl. Phys. A 205, 20 (1973); J. Maillet, J. P. Dedonder and C. Schmit, Nucl. Phy's. A 316, 267 (1979); W. R. Gibbs, in Theoretical Methods in Medium-Energy and Heavy Ion Physics, K. W. McVoy and W. A. Friedman, Eds. (Plenum Publishing Corporation, 1978), p. 503; M. Silver and N. Austern, Phys. Rev. C21, 272 (1980).

31. H. Garcilazo and W. R. Gibbs, Nucl. Phys. A 356, 284 (1981); W. R. Gibbs, B. F. Gibson, and G. J. Stephenson, Jr., Phys. Rev. Lett. 39, 1316 (1977).

32. J. T. Londergan, K. W. McVoy, and E. J. Moniz, Ann. Phys. (NY) 86, 147 (1974); M. J. Reiner, Phys. Rev. Lett. 38, 1467 (1977).

33. C. Schmit, Nucl. Phys. A 197, 449 (1972); C. Schmit, J. P. Dedonder, and J. P. Maillet, Nucl. Phys. A 239, 445 (1975); J. P. Maillet, J. P. Dedonder, and C. Schmit, Nucl. Phys. 271253 (1976).

34. W. B. Cottingame and D. B. Holtkamp, Phys. Rev. Lett. 45, 1828 (1y80).

35. R. H. Landau and A. W. Thomas, Phys. Lett. 61b, 361 (1976).

36. R. H. Landau and M. McMillan, Phys. Rev. C8, 2094 (1973); E. Kujaws'is and G. A. Miller, Phys. Rev. C9, 1205 (1974); G. A. Miller, Phys. Rev. C10, 1242 (1974'. R. Landau, Phys. Lett. 57B, 13 (1975).

37. W. R. Gibbs, B. F. Gibson, A. T. Hess, G. J. Stephenson, Jr., and W. B. Kaufmann, Phys. Rev. C 13, 2433 (1976).

38. A. Kerman, H. McManus, and R. Thaler, Ann. Phys. 8, 551 (1959).

39. R. H. Landau and A. W. Thomas, Nucl. Phys. A 302, 461 (1978).

40. P. B. Siegel, Ph.D. thesis, Arizona State University (1986); M. J. Leitch, H. W. Bacr, R. L. Burman, C. L. Morris, J. M. Knudson, J. R. Comfort, D. H. Wright, R. Gilman, S. H. Rokni, E. Piasctzky, Z. Weinfeld, W. R. Gibbs, and W. B. Kaufmann, Phys. Rev. C 39, 2356 (1989). 
41. J. P. Dctonder, J. P. Maillet, and C. Schmit, Ann. Phys. 127, 1 (1980).

42. R. V. Reid, Ann. Phys. (NY) 50, 411 (1968); B. D. Day, Phys. Rev. 24, 1203 (1981).

43. S. A. Coon, M. D. Scadron, P. C. McNamee, B. R. Barrett, D. W. E. Blatt, and B. H. J. McKellar, Nucl. Phys. A 317, 242 (1979).

44. R. B. Wiringa, R. A. Smith, and T. L. Ainsworth, Phys. Rev. C 29, 1207 (1984),

45. H. T. Coclho, T. K. Das, and M. R. Robilotta, Phys. Rev. C 28, 1812 (1983).

46. G. R. Smith, D. R. Gill, D. Ottewell, G. D. Wait, P. Walden, R. R. Johnson, R. Olszewski, R. Rui, M. E. Sevior, R. P. Trelle, J. Brack, J. J. Kraushaar, R. A. Ristinen, H. Chase, E. L. Mathie, V. Pafilis, R. B. Schubank, N. R. Stevenson, A. Rinat, and Y. Alexander, Phys. Rev. C 38, 240 (1989). 


\section{Report of the T.5 Theoretical Group}

L. Heller (Los Alamos)
Briefly summarized here are a few of the research topics considered during 1989 by members of the Medium Energy Physics Theory Group (T-5) of the Theoretical Division at Los Alamos. These topics span a wide range of subject matter from conventional nuclear physics to plysics of direct interest to LAMPr: and to problems that could be addressed with a LAMPF upgrade.

\section{The Anomalous Muon Decay $\mu^{+} \rightarrow e^{+} \bar{\nu}_{e} \nu_{\mu}$}

The decay $\mu^{+} \rightarrow e^{+} \bar{\nu}_{e} \nu_{\mu}$ is forbidden in the minimal standard model since it violates the conservation of additive electron and muon numbers. We pointed out (in collaboration with R. N. Molapatra) that this decay is of interest to an attractive class of $S U(2)_{l} \times S U(2)_{R} \times U(1)$ models, containing Higgs bosons belonging to triplet representations of $S U(2)_{L}$ and $S U(2)_{R} \cdot S U(2)_{L} \times S U(2)_{R} \times U(1)$ models with triplet Higgs bosons can account for the smallness of the masses of the usual neutrinos. The decay $\mu^{+} \rightarrow e^{+} \bar{\nu}_{e} \nu_{\mu}$ can occur through the exchange of the the singly charged component $\left(\Delta_{L}^{+}\right)$of the Higgs triplet coupled to the left-handed leptons.

The present upper limit for the $\mu^{+} \rightarrow e^{+} \bar{\nu}_{e} \nu_{\mu}$ branching ratio comes from a LAMPF experiment, which detected neutrinos from muon decay. The $\mu^{+} \rightarrow$ $e^{+} \bar{\nu}_{e} \nu_{\mu}$ branching ratio due to $\Delta_{L}^{+}$-exchange can be as large as the present limit. This decay mode provides information on left-right symmetric models that is complementary to the information obtained from searches for muoniumantimuonium conversion. The latter process is mediated by the doubly charged component of the Higgs triplet.

\section{Leptoquarks and Beta Decay}

Leptoquarks are bosons that induce quark-lepton transitions. They appear in many extensions of the minimal standard model. In models where they do not induce proton decay, leptoquarks could be light enough to cause observable effects in some low-energy proresses. We investigated the effects of leptoquark exchange in nuclear beta decay.

Leptoquark exchange gives rise to new vector and axial-vector beta-decay interactions. One of our results is that the exchange of $Q$ ( $\equiv$ electric charge) $=$ $-1 / 3$ spin-one leptoquarks and of $Q=2 / 3$ spin-zero leptoquarks give a contribution to the time-reversal-violating component of the $D$ "coefficient (the coefficient of the time-reversal-odd $\langle\vec{J}\rangle \cdot \vec{p}_{e} \times \vec{p}_{\nu} / J E_{e} E_{\nu}$ correlation). As this contribution is not constrained by the electric dipole moment of the neutron, nor by CP-violation in $k^{0} \rightarrow 2 \pi$, decays, it can be as large as the present experimental upper limit on $D$.

Both spin-one and spin-zero leptoquark exchange generate scalar-type betadecay interactions, while spin-zero leptoquark exchange also generates tensortype interactions. We find that, barring some cancellations, the experimental 
value of the ratio $\mathrm{I}(\pi \rightarrow \sigma \nu) / \mathrm{\Gamma}(\pi-\mu \nu)$ sets stringent limits on the strength of these interactions.

\section{Eta-Nucleon Cross Sections}

An analysis of inclusive production of etas from ${ }^{4} \mathrm{He},{ }^{7} \mathrm{Li},{ }^{12} \mathrm{C},{ }^{40} \mathrm{Ca}$, and ${ }^{210} \mathrm{~Pb}$ at a $\pi^{+}$beam momentum of $680 \mathrm{MeV} / \mathrm{c}$ has been completed by means of an intranuclear cascade. Since the eta production actually occurs only from the neutrons in the target, the data show a pronounced enhancement for the neutron "rich" elements over the isospin-zero nuclei. This feature is well reproduced by the analysis. The eta-nucleon cross section is separated into three pa.ts:

1. The $\eta N \rightarrow \pi N$ cross section, which is the inverse of the formation process and hence is moderately well known;

2. The $\eta N-\eta N$, i.c., clastic-scattering cross section, which is totally unknown in this energy region; and

3. The $\eta N \rightarrow X$, where $X$ is some other channel than those contained in Item 1 or 2 (c.g., $\eta N \rightarrow \pi \pi N$ ), also totally unknown.

From the analysis of the present data, the sum $\sigma_{1}+\sigma_{2}$ has been determined to be $25 \pm 5 \mathrm{mb}$. The separate cross sections cannot be reliably quoted individually, although there is a preference for most of the cross section to be in the inelastic channel. A measurement of inclusive pion double charge exchange through the intermediate cta channel (distinguishable from other processes by its kinematic characteristics) would allow a definitive separation of the two components.

\section{Parity-Violating Longitudinal Asymmetry in Proton-Proton Scattering}

The weak interaction between two protons has been detected at LAMPF by measurement, in scattering at $800 \mathrm{MeV}$, of the dependence of the total cross section on the helicity of the incident beam protons, a pari:y-violaing ef ect. At intermediate energies, there can be a (parity violating) pion-exchange contribution to the observed asymme'ry, $A_{L}=(2.4 \pm 1.0) \times 10^{-7}$, through production of the (doubly charged) $\Delta$ resonance.

For some time now we have been developing a calculation of this effect in a distorted-wave Born approximation description, dressing the weak $N N \rightarrow N \Delta$ amplitude with the (coupled) $N N \rightarrow N N$ and $N \Delta$ strong interaction amplitudes we computed earlier in a three-body model.

The first phase of this work has now been published. We have found that the pion-exchange contribution is significant, i.e., of the same size and magnitude as the earlier predictions of contribution to this asymmetry coming from $\rho$ and $\omega$ exchange. Unfortunately, with the large error bars on both the present data point and the theory (there is a sizable model dependence in the predicted value 
of both contributions), one is unable to say anything definite about the value of the (controversial) weak $N N \pi$ coupling constant; both the so-called "best value" and the much smaller values suggested by experiments on ${ }^{18} \mathrm{Ne}$ are compatible with the LAMPF datum.

It is of interest to other experiments of this type, such as the TRIUMF experiment in progress at $225 \mathrm{MeV}$ (i.e., below the pion-production threshold), that the pion exchange contributes even to an elastic-scattering asymmetry.

In more recent developments, we have improved our carlier calculation by using a more realistic strong-interaction model for the DWBA calculation. This model, using a two-body coupled channels quasi-potential approach, gives a much better representation of the $J^{P}=2^{ \pm}$strong-interaction partial-wave amplitudes, which basically determine the size of the effect. Combining this with the most recent calculation of the $\rho-\omega$ contribution, gives a total theoretical prediction for $A_{L}$ of about $1.0 \times 10^{-7}$.

\section{Parity Conserving Time-Reversal Violation in the Nucleon-Nucleon Interaction}

Recently we, and independently J. Kambor, M. Simonius, and D. Wyler, have shown that in a renormalizable gauge theory with elementary quarks, a coupling of a boson of any kind to fermion pairs camiot generate a flavor-conscrving ( $\Delta F$ $=0$ ) parity-conserving time-reversal-violating (PCTV) quark-quark interaction to second order in the boson-fermion couplings. A consequence of this is that PCTV in the nucleon-nucleon interaction in such models is generally expected to be unobservably small.

We have investigated now (in collaboration with J. Kambor, M. Simonius, and D. Wyler) PCTV in the $\Delta F=0$ quark-quark interaction, allowing the quarks to be composites of preons. We have shown that in some composite models $\Delta F=0$ PCTV quark-quark interactions can arise in second order in the bosonfermion couplings. We find that in such models PCTV nucleon $\rightarrow$ nucleon + meson vertices of strength not far from the prosent upper lirnils cannot be ruled out.

\section{Quantum Electro Dynamics (QED) Corretions to Quantum Chromo Dynamics (QCD) and Charge Symmetry Breaking}

Viewed from QCD, traditional approaches to charge symmetry breaking (CSB) have not included all electromagnetic corrections to the strong interactions. In particular, QED corrections to quark-gluon vertices affect this coupling differently for the different charge quarks, proportional to the squares of their charges. This effect is not described by form factors or meson mixing in traditional pictures that ignore the quark substructure of hadrons. It is however, 
analogous to the QED corrections to the pion-nuclcon coupling as discussed by Henley and Morrison. ${ }^{1}$

After identifying this effect, we analytica'ly calculated its expected size in the perturbative QCD region, and estimated it to be on the order of a few $\mathrm{MeV}$ for typical hadronic systems. Unfortunately, the perturbative $Q C D$ region does not overlap with the typical scales in hadronic bound states. A separate argument, based on the expected operator structures fitted to the charged and neutral pion mass difference (assuming sufficient proximity to the chiral limit), shows that matrix elements of order $2 \mathrm{MeV}$ are demanded by the data.

We have also applied these operator structures to a generol study of the pseudoscalar-meson mass splittings in the chiral limit, to baryon isomultiplet mass splittings, and to CSB in the $A=3$ nuclear system:

1. In the pscudoscalar mesors, the presence of these new operators significantly increases the uncertainty in the extraction of the light quark mass difference from data: the up-quark mass may range from virtually zero to equality with the down-quark mass. This is essentially due to the increased number of terms that must be included in the effective Lagrangian chiral perturbation expansion, as they cannot be argued to be negligible.

2. Using the nonrelativistic quark model of Isgur to control second-order effects, we find that these new operators also significantly affect the light-quark masses extracted from the baryon isomultiplet mass splittings. Due to the Coleman-Glashow relations, only three combinations of the four new matrix elements may be determined by fitting to the octet and decuplet splittings. We find that the down quark may be no more than $3 \mathrm{MeV}$ h avier than the up quark, and may be as much as $1 \mathrm{MeV}$ lighter. The result is sensitive to the mass difference between the positively and negatively charged $\Sigma^{*}$ baryons, the experimental value of which is presently in flux.

3. A $p-\omega$ mixing model oi CSB can accommodate the usual discrepancy between the measured and calculated binding energy difference in the $A=3$ nuclear isodoublet only if one ignores the reduction in the mixing matrix element between the experimentally measured value at a time-like momentum and the space-like momentum values needed for meson exchange in nuclei. Maltman has observed that nucleon-quark substructure effects worsen the $A=3$ discrepancy, which further strains the rho-omega mixing model. Considering only Pauli effects in a quarkcluster model for these nuclei, we find that significant contributions to CSB in the $A=3$ system from the new CSB-quark operators cannot be ruled out. The precise amount depencis nontrivially upon that combination of matrix elements not determined by fits to the baryon ociet and decuplet isomultiplet splittings. The isomultiplet mass splittings of the chamed baryons, however, could be used to constrain this quantity. 
We will apply our relativistic quark model of nuclei to the $A=3$ system (and to the $A=4$ hypernuclear system) to see how much these result are affected by the quark delocalization, which produces nuclear binding in that model.

\section{$\Lambda N-\Sigma N$ Coupling in ${ }_{\Lambda}^{3} \mathrm{H}$}

The hypertriton plays the important role in hypernuclear physics that the deuteron plays in conventional (strangeness zero) nuclear physics. Neither the $\Lambda N$ nor $\Sigma N$ interactions possess sufficient strength to support a bound state. It is the ground state $\left(J^{\pi}=1 / 2^{+}\right)$of the $\Lambda N N$ system that is utilized to constrain our models of the hyperon-nucleon $(\mathrm{YN})$ force $\cdots$ models that are not otherwise well determined by the sparse data base for low-energy $\Lambda N$ and $\Sigma N$ scattering and reactions. Because the hypertriton is loosely bound $\left[B_{\Lambda}=B\left({ }_{\Lambda}^{3} \mathrm{H}\right)-B\left({ }^{2} \mathrm{H}\right) \simeq\right.$ $-0.13 \pm 0.05 \mathrm{MeVl}$, one expects this molecular-like system to be most sensitive to the long-range aspects of the $\mathrm{YN}$ force. However, there is no one-pion-exchange mechanism allowed in the $\Lambda N$ channel because $\Lambda(T=0)$ and $N(T=1 / 2)$ cannot ex zhange a $T=1$ pion. The allowed $\bar{K}$-exchange potential does admit a tensor force component, but that is largely cancelled by the $K^{*}$-exchange potential. (The $\pi$-exchange and $p$-exchange tensor forces in the $N N$ interaction do not cancel so completely because their masses are quite different.) Thus, tensor force effects in the $\Lambda N$ interaction are anticipated to be smaller than those in the $N N$ interaction. In contrast, $\Lambda N-\Sigma N$ coupling effects are expected to be much more important in hypernuclear physics than are $N N-N \Delta$ coupling effects in conventional nuclear physics. The $M_{\Sigma}-M_{\Lambda}$ mass difference is only $75 \mathrm{MeV}$, and the width of the $\Sigma$ is small compared to that of the $\Delta$ because the $\Sigma$ lies below the $K^{-} p$ threshold. Freezing out, or formally eliminating the $\Sigma N$ channel from the problem, leads one to $\Lambda N N$ three-body forces.

Using separable potential Faddeev-type equations to model the hypertriton, we have explored tensor force and $\Lambda N-\Sigma N$ coupling in that three-body system. Tensor force effects are shown to be nonperturbative, but small. Explicit $\Lambda N-\Sigma N$ coupling is shown to play a significant role. When the $\Sigma N$ channel is formally eliminated, the dispersive energy dependence of the resulting $\Lambda N$ interaction is repulsive, whereas the resulting $\Lambda N N$ three-body force is attractive. One is led to ask whether the hypertriton is bound only because there is a $\Lambda N N$ three-body force.

\section{Nucleon-Deuteron-Scattering Lengths}

The scattering of nucleons from deuterons at very low energies is the next most complicated three-nucleon calculation after the bound states. The first example of trinucleon "scaling" was observed in calculations of doublet neutrondet eron scattering at vanishingly small encrgies. Calculated values of the scattering length and triton binding energy for a varicty of simple potential models were found to track a nearly universal (Phillips) curve. More recently, we showed 
that a different such curve also determines the Coulomb-modified 1 -d doublet scattering lengths, when plotted versus the ${ }^{3} \mathrm{He}$ binding energy.

All of the early calculations involved schematic force models, or models truncated to the lowest few partial waves. We have recently completed an extensive study of "exact," or numerically complete, solutions of the scattering length problem, keeping a very large (sufficient) number of partial waves. This recent work complements our previous "exact" solutions for the bound-state problem. The nuclear-force models that were used include a variety of "realistic" nucleonnucleon potentials, with tensor forces and strong short-range repulsion, together with models that additionally include three-nucleon forces. These calculations demonstrate that a Phillips' curve also applies to the complete solutions over a very wide range of trinucleon binding energies. The calculated $n-d$ scattering lengths, evaluated for the physical tritun binding energy, are in good agreement with experiment. The $r$-d scattering length is not, because of severe difficulties associated with extrapolating the rapidly varying experimental pha e shifts over a large energy interval.

\section{Thermal $n+d \rightarrow{ }^{3} \mathrm{H}+\gamma$}

The exchange of mesons between nucleons is the physical mechanism that mediates the nucleon-nucleon force in the meson-baryon picture of the itrong interaction. These meson-exchange currents also modify the electromagnetic properties of a nucleon bound in a nucleus, compared to its electromagnetic properties in free space. Two examples that clearly demonstrate the need to include meson degrees of freedom in our description of nuclei in terms of the physically observable hadrons are thermal $n-p$ radiative capture and the magnetic moments of the trinucleoris. In the former case, the experimental cross section is $334 \mathrm{mb}$ compared to the theoretical impulse approximation (nucleons only) value of some $300 \mathrm{mb}$. Meson-exchange-current corrections are a nonnegligible $10 \%$. In the latter case, the ${ }^{3} \mathrm{He}$ and ${ }^{3} \mathrm{H}$ magnetic moments are 2.1275 and 2.9789 nuclear magnetons, respectively, compared to impulse approximation values of 1.75 and 2.55. The difference between these values shows a clear $20 \%$ meson-exchangecurrent enhancement.

The threshold $n+d \rightarrow{ }^{3} \mathrm{H}+q$ reaction provides a process in which mesonexcuange-current effects are not just a correction but the dominant mechanism. This magnetic-dipole transition is small $(0.508 \pm 0.015 \mathrm{mb})$ because the primary $S-S$ transition, which dominates the two examples above, vanishes. This results from the required orthogonality of the initial and final spin-doublet states and because of the mixed-symmetry spatial composition of the spin-quartet continuum. The impulse-approximation cross section arises from the small mixedsymmetry components of the ground-state wave function, and the mesonexchange-current contribution to the reaction can be larger than the impulseapproximation result. We have undertaken a calculation of the nd radiative 
capture cross section using thee-body ground-state and continuum wave functions generated from the Los Alamos-Iowa configuration-space Faddeev codes. Employing contemporary nucleon-nucleon and three-nucleon force models, we incorporate proper three-body dynamics in both initial and final states. Our meson-exchange-current model contains pion currents (true exchange), a pair or sea gull term, and a $\Delta$-isobar diagram. These pion-exchange-current corrections to the isovector electromagnetic current operator provided the enhancement necessary to account for both the $n+p \rightarrow d+\gamma$ thermal cross section and the trinucleon magnetic moments. Preliminary results indicate that our model calculations which reproduce the correct triton binding energy will properly account for the $n+d \rightarrow{ }^{3} \mathrm{H}+\gamma$ thermal cross section, implying that our meson-exchangecurrent model is adequate to describe isovector-exchange-current effects in a complex few-body reaction. Extension of these calculations to $p+d \rightarrow{ }^{3} \mathrm{H}+\gamma$ should also permit us to estimate the E0, M1, and E2 contributions to the muon catalyzed $p+d$ fusion reaction.

\section{Three Nucleon Interactions in the Alpha Particle}

During the past year we have extended our Green's function Monte Carlo (GFMC) studies of light nuclei to include realistic twc-pion-exchange models of the three-nucleon interaction. The three-body force is quite important in the alpha particle, since there are four triplets, compared to only one for $A=3$. Combined with the somewhat higher density of ${ }^{4} \mathrm{He}$, this means that the threebody force models typically provide 4 to $5 \mathrm{MeV}$ out of the total $28-\mathrm{MeV}$ binding energy.

Initial GFMC calculations of the alpha particle treated the three-body force perturbatively, and indicated that the alpha particle would be overbound by the Argonne nucleon-nucleon plus Urbana model 7 three-nucleon interaction (TNI). By reducing the strength of the two-pion-exchange component of the force, it is possible to provide a reasonable fit to both the three- and four-body nuclear ground states. Earlier models of TNI, whose strengths were determined by fits to $A=3,4$ binding energies obtained in variational calculations, actually overbind the alpha particle by several MeV.

We have also been employing GFMC methods to study other interesting aspects of aipha particle structure. For example, we have calculated the momentum distribution of the nucleons, as well as the charge form factor and the Coulomb sum rule. We are currently extending calculations of electromagnetic properties to include exchange currents. GFMC should provide valuable new tests for these models of exchange currer.ts.

\section{Radiative Capture of Neutrons on ${ }^{3} \mathrm{H}$}

We have developed a new method for calculating low-energy nuclear scattering employing variational Monte Carlo methods. It is possible to construct 
upper-bound variational principles for scattering states to the Schrödinger equation by employing the so-called eigenphase solutions. In principle, this method should allow us to investigate a large number of resonances in light nuclel. The first application of this method has been to study the radiative capture of thermal neutrons on ${ }^{3} \mathrm{He}$.

This reaction is particularly interesting because it is dominated to a large degree by exchange-current contributions. For purcly s-wave ground states the impulse approximation matrix element for this M1 transition is exactly zero, and even for the sizable $D$ states encountered in realistic wave functions the impulse approximation gives only about $10 \%$ of the experimental cross section.

Using the variational Monte Carlo methods, we have calculated a scattering length of $3.5 \pm 0.25 \mathrm{fm}$ for the neutron ${ }^{3} \mathrm{He}$ system, using realistic two- and three-nucleon interaction models. This compares well with the experimentally determined value of $3.5 \pm 0.25 \mathrm{fm}$, but is somewhat larger than the $3.25 \mathrm{fm}$ obtained with R-matrix techniques. With this wave function, we can then calculate the matrix of the current operator, which causes transitions to the alpha-particle ground state. Present-day models of exchange currents seem to overestimate the cross section, giving a cross section nearly twice the experimental value, $120 \mathrm{mb}$ as compared to the value of $60 \mathrm{mb}$ found in recent experiments. The matrix element, therefore, is overestimated by roughly $40 \%$. Of course, there are significant uncertainties in the exchange-current models arising from short-range form factors, coupling constants, etc. This reaction should be very valuable in helping us to understand these issues.

The radiative capture has also been the subject of considerable interest because of its possible relation to the weak capture of protons on ${ }^{3} \mathrm{He}$. This weak capture produces neutrinos with a high endpoint energy, which may be detectable in future solar-neutrino observatories. Because of the strong dependence of the radiative capture on exchange currents, the relationship between the two rates seems far from trivial. It should be feasible to investigate the weak capture with these same techniques, and we plan to do this in the near future.

\section{Exchange Currer:ts}

In many problems in nuclear physics, phenomenological form factors are used at vertices to represent the effect of higher-order processes. If charged particles are involved, then there is additional, i.e., exchange current within the vertex that must be taken into account in any electromagnetic process. The precise form of this current can only be determined by knowing the details of the strong ir.teraction; but for photon momenta that are not too large any reasonable current that--.-together with the current of the particles entering and leaving the vertex is conserved, will suffice. We has? found a direct way of exploiting the Ward identity to produce one particular contribution to the bremsstrahlung matrix element due to radiation from the vertex. It corresponds to the minimal current assignment for the particles inside the vertex, and nas been applicd to the 
$\pi N \Delta$ vertex in pion-nucleon bremsstrahlung. We plan to bring this and other results together into a mini-review article about exchange currents.

\section{Pion Double Charge Exchange from Nuclei}

Configuration admixtures in the calcium isotopes have been shown not to effect the dependence of the pion double charge exchange (DCX) to the doubleanalog states on the number of valence neutrons $n$, but does renormalize the basic double charge exchange $A$ and $B$ amplitudes used in the formula for the DCX amplitude $\tilde{i}^{-1}=[n(n-1) / 2]^{1 / 2}\{\tilde{A}+(\Omega+1-n) /(\Omega-1)(n-1) \widetilde{B}\}$, where $2 \Omega$ is the number of single-neutron states and $\tilde{A}, \widetilde{B}$ are the renormalized $A, B$ amplitudes.

As a consequence this formula remains useful for extracting phenomenologically the basic DCX two-neutron long- and short-range amplitudes $A$ and $B$, respectively, froin the $D C X$ ress sections with the various calcium isotopes as targets. Calculations of DCX on ${ }^{42} \mathrm{Ca}$ show that the cross section is sensitive to the different interactions used to calculate the ${ }^{12} \mathrm{Ca}$ wave functions, increasing the cross section by a factor from 1.4 to 1.7 over the pure configuration, depending on the interaction used. At $35 \mathrm{MeV}$, the measured cross section agrees with the interaction producing the least configuration admixture. At $292 \mathrm{MeV}$, the measured cross section agrees with the pure configuration. A possible conclusion is that the sequential reaction mechanism at $292 \mathrm{McV}$ is overestimating the short-range contribution to the cross section. The other calcium isotopes are now being calculated, including configuration admixing in perturbation theory. The pion double charge exchange to $J=0^{+}$states other than the double analog has also been calculated for the calcium targets as a function of energy. These states are the ground states and excited states in the residual titanium isotopes and have isospin two units less than the double analog state. DCX cross sections to these states have very different energy dependence. It is found that the excited state at about $10 \mathrm{MeV}$ in ${ }^{48} \mathrm{Ti}$ has a large contribution from the double Gamow-Teller strength of the DCX process.

\section{Strangeness Production in Antiproton-Nucleus Collisions}

By following the propagation of the strangeness produced in antiproton annihilation through the nuclear medium, including the strangeness created by the associated production reaction, we have calculated the rapidity distribution of the $\Lambda^{\prime} s$ and $K_{s}$ 's produced in the annihilation of antiprotons on Ta at a beam energy of $4 \mathrm{GeV} / c$ where there is data from KEK." We find that the calculations agree quite well with the experimental results. Hence, there is no need to pestulate the formation of a quark-gluon plasma in this experiment as has been claimed in the literature. We also can easily understand the factor of five in the number of lambdas produced, over what would occur in free space, from the strangeness exchange in the nuclear medium. The principal uncertainty in the 
calculation of the $\Lambda$ rapidity distribution arises from a lack of knowledge of the $\Lambda$-nucleon cross section.

\section{Neutron and Proton Degrees of Freedom in Collective Motion}

The study of the different contributions of neutrons and protons to nuclear collective motion is revealing new types of collective motion. The neutrons and protons in nuclei can each move collectively in quadrupole shapes, and the large neutron-proton interaction can cause these shapes to rotate together and generate the ground-state band of the nucleus. Usually it is assumed that both the neutrons and protons have axially symmetric prolate shapes, and in some cases, axially symmetric oblate shapes with the same deformation. However, it is more than likely that the neutrons and protons will have different deformation and for some nuclei it may be possible that one shape could be prolate and the other oblate. We are studying the spectroscopy of this type of collective motion with the aim of finding unique identifying features that can be observed experimentally. Also, there is the possibiiity that the quadrupole shapes can oscillate with respect to each other, producing excited isovector collective bands. One example of this type of motion is the "scissors" state, an isovector state with $J^{\pi}=1^{+}$, which is an orbital excitation as opposed to a spin excitation, and which has been observed in electron scattering. However, there is controversy as to whether this state is in fact an isovector orbital excitation. The positive and negative pion beams at LAMPF are ideally suited to study these new collective modes, the difference between neutron and proton deformations, and the new isovector excited modes since positive and negative pion scattering and charge exchange on nuclei can be used to extract the isovector properties of nuclear collective motion. In addition, pion scattering at small angles is dominated by orbital excitations and hence a separation of orbital and spin excitations is also possible.

Furthermore, there are experimental indications that nuclei in the $\mathrm{Xe}, \mathrm{Ba}$, and $\mathrm{Pt}$ regions may have a quadrupole shape without an axis of symmetry, i.e., neither oblate or prolate. These triaxial configurations arise if the collective Hamiltonian contains at least three-body interactions. A study is being carried out on the spectral significance of such higher-order terms. The new features observed are the mixing of the $\beta$ and $\gamma$ motions in the triaxial-intrinsic modes and a possibility for coexisting prolate minima with different doformations in the energy surface. The latter may be relevant fo. describing shape isomers and superdeformation.

\section{Group T-5}

Anyone wishing further information on these matters may contact Croup T'-5 members: J. A. Carlson, J. L. Friar, W. R. Gibbs (Group Leader), B. F. Cibson, J. N. Ginocchio, T. Goldman, L. Heller, P. Herczeg, and R. R. Silbar. A. Leviatan and 
A. Hayes held postdoctoral appeintments in T-5 during 1989. The long-term visitors during 1989 were J.-P. Dedonder (University of Paris-VII), W. B. Kaufmann (Arizona State University), G. L. Payne (University of lowa), J. A. Tjon (University of Utrecht), B. C. Pearce (TRIUMF), J. D. Vergados (University of Ioannina), 1. Talmi (Weizmann Institute of Science), B. Loiscau (CNRS/University of Paris), A. Arriaga (University of Lisbon), D. Wyler (PSI), A. Van Hees (Rijksuniversiteit Utrecht), and S. Kuyucak (University of Melbourne).

\section{References}

1. E. M. Henley and L. K. Morrison, Phys. Rev. 141, 1489 (1966); L. K. Morrison, Ann. Phys. (NY) 50, 6 (1968).

2. K. Miyano, Y. Noguchi, M. Fukawa, E. Kohriki, F. Ochiai, T. Sato et al., Phys. Rev. Lett. 53, 1725 (1984).

\section{Group T-5 Publications}

A. T. Acrts and L. Heller, "Excited States of the $\Omega^{-}$, , Phys. Lett. B 221, 194 (1989).

I. R. Afran and B. F. Gibson, " $\Lambda N-\Sigma N$ Coupling in ${ }_{\Lambda}^{3} \mathrm{H}$," Phys. Rev. C40, R7 (1989).

J. Carlson, "Green's Function Monte Carlo Calculations of Light Nuclel," presented at the Few Body XII Conference, Vancouver, British Columbia, July 1989, Nucl. Phys. A 508, 141 (1990).

J. Carlson, "CFMC Study of the Heisenberg Antiferromagnet," in Proceedings of the Quantum Simulations of Condensed Matter Phenomenon, Los Alamos, New Mexico, August 1989, J. D. Doll and J. E. Gubernatis, Eds. (World Scientific, Singapore, 1990), p. 31.

J. Carlson, J. L. Friar, and G. L. Payne, "Energy and Symmetry of States in Light Nuclel," Phys. Rev. C37, 420 (1988).

J. Carlson, Jules W. Moskowitz, and K. E. Schmidt, "Model Hamiltonians for Atomic and Molecular Systems," J. Chem. Phys. 90, 1003 (1989).

J. Carlson, D. O. Riska, R. Schiavilla, and R. B. Wiringa, "Radiative Neutron Capture on "He," Los Alamos National Laboratory document LA-UR-90-1224 [to be published in Physical Review C (1990)].

C. R. Chen, C. L. Payne, J. L. Friar, and B. F. Gibson, "Low-Energy NucleonDeutcron Scattering," Physs. Rev. C 39, 1261 (1989). 
J. Q. Chen, X. G. Chen, D. H. Feng, C. L. Wu, J. N. Ginocchio, and M. W. Guidry, "The $S p_{6} \times S U_{2}$ Symmetry of the Fermion Dynamical Symmetry Model," Phys. Rev. C 40, 2844 (1989).

F. Cooper, J. N. Ginocchio, and A. Wipf, "Supersymmetry, Hidden Shape Invariance and Exactly Solvable Potentials," I. Phys. A22, 3707 (1989).

W. R. Gibbs and B. F. Gibson, "Neutron Radius Analysis in the Trinucleon System from Pion Scattering," Los Alamos National Laboratory document LA-UR-90-438 [submitted to Physical Review C (1990)].

W. R. Gibbs and J. W. Kruk, "Strangeness Production in Antiproton-Tantalum

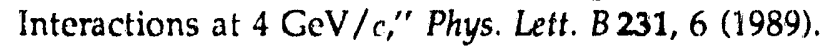

W. R. Gibbs, "Topics in Recent Pion-Nucleus Physics," Proceedings of the LAMPF Users Group, October 1988, Los Alamos National Laboratory report LA-11556-C (1989), p. 27.

W. R. Gibbs, W. B. Kaufmann and J. P. Dedonder, "The Pion-Nucleus Resonance and Nuclear Translucence," Phys. Lett. B 231, 6 (1989).

W. R. Gibbs and J. W. Kruk, "High-Energy Density from Antinucleon-Nucleus Collisions," invited talk at "The Physics to be Done at KAON," Bad Honnef, West Germany, June 9-11, 1989, and Z. Phys. C 46, 545 (1990).

B. F. Gibson, "Decay of Hypernuclei," in 1988 International Symposium on Hypernuclei and Low-Energy Kaon Physics, Padova, Italy, September 1988, T. Bressani, Ed. [Nuovo Cimento 102A, 367 (1989)].

B. F. Gibson, B. C. Pearce and G. L. Payne, "Triton Model Calculations Test of the Bonn W'-Matrix Rank-One Approximation," Phys. Rev. C 40, 2877 (1989).

J. N. Ginocchio, "The Effect of Configuration Admixtures on Pion Double Charge Exchange," Phys. Rev. C 40, 2168 (1989).

T. Goldman, K. R. Maltman and G. J. Stephenson, Jr., "The Finite QED Correction to the Quark-Gluon Yertex," Phys. Lett. B 228, 396 (1989).

A. C. Hayes, "Nuclear Structure and Pion Reactions," invited talk at the "Second LAMPF Workshop on Pion-Nucleus Double Charge Exchange," Los Alamos, New Mexico, August 9-11, 1989.

A. C. Hayes, J. L. Friar, and D. D. Strottman, "Two-Photon Decay of the First Excited 0+ State in ${ }^{16}$ O," Bull. Am. Phys. Soc. 34, 1187 (1989). 
L. Heller and W. R. Gibbs, " $\pi$ - Capture on the Deuteron as a Dibaryon Search," Phys. Rev. C 39, 1896 (1989).

L. Heller, S. Kumano, J. C. Martinez, and E. J. Moniz, "Reply to 'Comment on 'Pion-Nucleon Bremsstrahlung and $\Delta$ Electromagnetic Moments' '," Phys. Rev. C 40, 2430 (1989).

P. Herczeg, "Exotic Decays of Light Mesons," in Production and Decay of Light Mesons, P. Flcury, Ed. (World Scientific, Sirigapore, 1988), p. 16.

P. Herczeg, "T-Violation in Nuclear Interactions-An Overview," Hyp. Int. 43, $61(1988)$.

P. Herczeg, "Pion and Muon Decays Beyond the Standard Model," in Rare Decays Symposium, D. Bryman, J. Ng, T. Numao, and J.-M. Poutissou, Eds. (World Scientific, Singapore, 1989), p. 24.

P. Herczeg, "Beta-Decay Beyond the Standard Model," invited talk at the International Symposium on Fundamental Symmetries in Nuclei and Particles, Caltech, Pasadena, California, September 7-9, 1989, Los Alamos National Laboratory document LA-UR-89-4172 Ito be published in the proceedings (World Scientific, Singapore)] (cf. PUBL:33).

W. B. Kaufmann and W. R. Gibbs, "Corrclation, Nuclear Structure, and DCX," invited presentation at the "Second LAMPF Workshop on P'ion-Nucleus Double Charge Exchange," August 9-11, 1989.

W. B. Kaufmann and W. R. Gibbs, " $K^{+}$Nucleus Total Cross Section Analysis," Phys. Rev. C 40, 1729 (1989).

W. M. Kloct, R. R. Silbar, and J. A. Tjon, "Parity-Violating Asymmetry in $\vec{p}$ p Scattering at Medium Energies," Phys. Rev. C41, 2263 (1990).

M. J. Leitch, H. W. Bacr, R. L. Burman, C. L. Morris, J. N. Knudson, J. R. Comfort, D. H. Wright, R. Gilman, S. H. Rokni, E. Piasetzky, Z. Weinfeld, W. R. Gibbs, and W. B. Kaufmann, "The ${ }^{14} \mathrm{C}\left(\pi^{+}, \pi^{-}\right)^{14} \mathrm{O}$ Reaction Between 19 and $80 \mathrm{MeV}$," Phys. Rev. C 39, 2356 (1989).

A. Leviatan, "Intrinsic and Collective Patterns in Algebraic Bosonic Models," Bull. Am. Phys. Soc, 34, 1834 (1989).

A. Leviatan, "Point Groups in the Vibron Model," I. Chem. Phys. 91,1706 (1989). 
A. Leviatan and M. W. Kirson, "Normal Modes of the Proton-Neutron Interacting-Boson Model of Nuclei," Ann. Phy. (NY) (1990).

A. Leviatan and B. Shao, "Deformed Single-Particle Levels in the Boson-Fermion Model," Phys. Rev. Lett. 63, 2204 (1989).

K. Maltman, T. Goldman, and G. J. Stephenson, Jr., "Corrections to the Extraction of Light Quark Mass Ratios from Pscudoscalar Meson Splittings," Phys. Lett. B 234, 158 (1990).

R. M. Panoff and J. Carlson, "Fermion Monte Carlo Algorithms and Liquid " $\mathrm{He}$," Phys. Rev. Lett. 62, 1130 (1989).

B. C. Pearce and B. F. Gibson, "Observable Effects of Poles and Shadow Poles in Coupled Channel Systems," Phys. Rev. C 40, 902 (1989).

R. L. Shypit, D. V. Bugg, A. H. Sanjari, D. M. Lee, M. W. McNaughton, R. R. Silbar, C. L. Hollas, K. H. McNaughton, P. Riley, and C. A. Davis, "Spin Dependence of $p p^{\prime-\ldots} n \pi^{+}$from 492 to $796 \mathrm{MeV}$," Phys. Rev. C 40, 2203 (1989).

R. R. Silbar, W. M. Kloet, L. S. Kisslinger, and J. Dubach, "Pion-Exchange Contribution to the Parity-Violating Asymmetry in $\vec{p}$, Scattering," Phys. Rev. C40, 2218 (1989).

G. Wenes and J. N. Ginocchio, 'Random Phase Approximation in the Fermion Dynamical Symmetry Model: SO(8)," Phys. Rev. C 39, 2426 (1989).

H. T. Williams and R. R. Silbar, "Automated Angular Momentum Recoupling Algebra" [subinitted to the I. Comp. Phys. (May 1990)].

W. M. Zhang, D. H. Feng, C. L. Wu, H. Wu, and J. N. Ginocchio, "Symmetry Constrained Hartree-Fock-Bogolyubov Theory," Nucl. Phys. A 505, 7 (1989). 


\section{MP.DIVISION PUBLICATIONS}

\section{Publications}

\section{Nuclear and Particle Physics Research}

K. Abe, E. W. Beier, L. S. Durkin, A. K. Mann, F. M. Newcomer, L. A. Ahrens, S. H. Aronson, B. G. Gibbard, M. J. Murtagh, S. Terada, K. Amako, S. Kabe, T. Shinkawa, J. L. Callas, D. Cutts, M. V. Diwan, J. S. Hoftun, R. E. Lanou, D. Hedin, M. D. Marx, E. Stern, Y. Kurihara, Y. Nagashima, Y. Suzuki, Y. Yamaguchi, and D. H. White, "Determination of $\sin ^{2} \theta_{W}$ from Measurements of Differential Cross Sections for Muon Neutrino and Anti-Neutrino Scattering by Electrons," Phys. Rev. Lett. 62, 1709 (1989).

G. S. Adams, B. Hoistad, J. M. Laget, B. Aas, K. Jones, J. A. McGill, G. S. Blanpied, C. S. Mishra, G. Pignault, C. S. Whisnant, and G. Pauletta, "Analyzing Power for $\vec{p}+d \rightarrow{ }^{3} \mathrm{He}+\pi^{0}$ at $800 \mathrm{MeV}, "$ Phys. Lett. B 219, 399 (1989).

K. Akchurin, D. Carey, R. Coleman, M. D. Corcoran, J. D. Cossairt, A. Derevschikov, M. M. Gazzaly, D. P. Grosnick, D. Hill, K. Imai, K. Kuroda, M. Laghai, F. Lehar, A. de Lesquen, D. Lopiano, F. C. Luchring, A. Maki, S. Makino, A. Masaike, Yu. A. Matulenko, A. P. Meschanin, A. Michalowicz, D. H. Miller, K. Miyake, T. Nagamine, F. Nessi-Tedaldi, M. Nessi, C. Nguyen, S. B. Nurushev, Y. Ohashi, Y. Onel, G. Pauletta, A. Penzo, G. C. Thillips, A. L. Read, J. B. Roberts, L. van Rossum, G. Salvato, P. Schiavon, T. Shima, V. L. Solovyanov, H. Spinka, R. W. Stanek, R. Takashima, F. Takeuchi, N. Tamura, N. Tanaka, D. G. Underwood, A. N. Vasiliev, A. Villari, J. L. White, A. Yokosawa, T. Yoshida, and A. Zanetti, "Analyzing-Power Measurements of Coulomb-Nuclear Interference with the Polarized-Proton and -Antiproton Beams at $185 \mathrm{GeV} / c_{,}^{\prime \prime}$ Nucl. Instrum. and Methods B 229, 299 (1989).

M. Akei, J. F. Amann, J. Chiba, H. Ejiri, Y. Iseki, M. Fukuda, T. Fukuda, O. Hashimoto, S. Homma, T. Irie, A. Kashitani, S. Kato, K. Kimura, T. Kishimoto, K. Maeda, Y. Matsuyarna, J. A. McGill, T. Nagae, H. Nagasawa, C. Hagoshi, H. Noumi, M. Nomachi, H. Ohsumi, K. Okuda, K. Omata, H. Sonog, O. Sanog, O. Sasaki, T. Shibata, F. Soga, F. Takeuchi, H. A. Thiessen, S. Toyama, K. H. Tanaka, Y. Umeda, Y. Yamanoi, and N. Yoshikawa, "Study of Hypernuclei via $\left(\pi^{+}, K^{+}\right)$Reactions at KEK,' Institute for Nuclear Study, Tanashi, Tokyo 188, Japan, INS-Rep.-747 (May 1989).

M. Akei, H. Ejiri, M. Fukuda, T. Irie, Y. Iscki, A. Kashitani, T. Kishimoto, H. Nagasawa, H. Noumi, H. Oshumi, K. Okuda, Y. Umeda, T. Kududa, T. Shibata, O. Hashimoto, S. Homma, Y. Matsuyama, T. Nagae, C. Nagoshi, K. Omata, F. Soga, S. Toyama, N. Yoshikawa, Y. Yamanoi, J. F. Amann, J. A. McGill, H. A. Thiessen, J. Thiba, M. Nomaciri, O. Sasaki, K. H. Tanaka, S. Kato, K. Kimura, F. Takeuchi, and K. Maeda, "Study of ${ }^{12} \mathrm{C}$ and ${ }^{56} \mathrm{Fe}$ Hypernuclei by $\left(\pi^{+}, K^{+}\right)$ Reactions at the KEK 12-GeV PS," Nuovo Cimento 102, 457 (1989). 
M. Akei, J. F. Amann, J. Chiba, H. Ejiri, M. Fukuda, T. Fukuda, O. Hashimoto, S. Homma, T. Irie, Y. Iseki, A. Kashitani, S. Kato, K. Kimura, T. Kishimoto, K. Maeda, Y. Matsuyama, J. A. McGill, T. Nagae, H. Nagasawa, C. Nagoshi, M. Nomachi, H. Noumi, K. Okuda, K. Omata, H. Sano, O. Sasaki, T. Shibata, F. Soga, F. Takeuchi, H. A. Thiessen, S. Toyama, K. H. Tanaka, Y. Yamanoi, and N. Yoshikawa, "A Broad-Range Spectrometer PIK for Medium-Energy Muon Spectroscopy," Laboratory of Nuclear Studies, Osaka University, Toyonaka, Osaka 560, Japan, OULNS 88-11 (1988).

D. E. Alexandreas, R. C. Allen, S. Biller, G. Dion, X-Q. Lu, P. R. Vishwanath, G. B. Yodh, R. L. Burman, D. R. Cady, C. M. Hoffman, J. Lloyd-Evans, D. E. Nagle, M. E. Potter, V. D. Sandberg, C. A. Wilkinson, D. Berley, C. Y. Chang, B. L. Dingus, J. A. Goodman, T. J. Haines, D. A. Krakauer, M. Stark, and R. L. Talaga, "Relative Muon Content of UHE Showers Associated with Hercules X-1," in Proceedings of the XXI International Cosmic Ray Conference, Adelaide, Australia, January 6-19, 1990, R. J. Protherce, Ed. (University of Adelaide, Physics Publications, 1989), Code OG 4.2-8, Vol. 2, p. 116.

R. C. Allen, V. Bharadwaj, G. A. Brooks, H. H. Chen, P. J. Doe, R. Hausammann, W. P. Lee, X-Q. Lu, H. J. Mahler, M. E. Potter, A. M. Rushton, K. C. Wang, T. J. Bowles, R. L. Burman, R. D. Carlini, D. R. F. Cochran, J. S. Frank, E. Piasetzky, V. D. Sandberg, D. A. Krakauer, and R. L. Talaga, "Measurement of the Exclusive Reaction $\nu_{e}+{ }^{12} \mathrm{C} \rightarrow E^{-}+{ }^{12} \mathrm{~N}($ g.s.)," in Proceedings of the American Physical Society Division of Particles and Fields Meeting, Storrs, Connecticut, August 15-18, 1988, pp. 696-697.

R. C. Allen, H. H. Chen, P. J. Doe, R. Hausammann, W. P. Lee, H. J. Mahler, M. E. Potter, X. Q. Lu, K. C. Wang, T. J. Bowles, R. L. Burman, R. D. Carlini, D. R. F. Cochran, J. S. Frank, E. Piasetzky, V. D. Sandberg, D. A. Krakauer, and R. L. Talaga, "Measurement of Interference between $W$ and $Z$ Exchange in Electron-Neutrino Eleciron Scattering," Phys. Rev. Lett. 64, 1330 (1990).

R. C. Allen, H. H. Chen, M. E. Potter, R. L. Burman, J. B. Donahue, D. A. Krakauer, R. L. Talaga, E. S. Smith, and A. C. Dodd, "A Measurement of the Neutrino Flux from a Stopped Pion Source," Nucl. Instrum. and Methods A 284, 347 (1989).

P. Astier, G. Bernardi, G. Carugno, J. Chauveau, P. Chrisicopoulou, J. Dumarchez, M. Ferro-Luzzi, F. Kovacs, A. Letessier-Selvon, J.-M. Levy, M. J. Murtagh, J.-M. Perreau, Y. Pons, J. L. Stone, A.-M. Touchard, F. Vannucci, P. Wanderer, and D. H. White, "Search for Neutrino Oscillations," Phys. Lett. B 220, 646 (1989). 
P. Astier, G. Bernardi, G. Carugno, J. Chauveau, P. Chrisicopoulou, J. Dumarchez, M. Ferro-Luzzi, F. Kovacs, A. Letessier-Selvon, J.-M. Levy, M. J. Murtagh, J.-M. Perreau, Y. Pons, J. L. Stone, A.-M. Touchard, F. Vannucci, P. Wanderer, and D. H. White, "A Search for Neutrino Oscillations," Los Alamos National Laboratory document LA-UR-89-3319 and CERN-EP/89-128 (submitted to Nuclear Physics, September 1989).

M. Atiya, E. Blackmore, G. Bock, D. Bryman, M. Cooper, H. Gordon, L. Littenburg, W. Louis, H. Lubatti, K. McFarlane, K. Nishikawa, J. Ritchie, L. Roberts, T. Shinkawa, M. Sivertz, A. J. S. Smith, G. Thomson, R. Tschirhart, and T. Yamanaka, "Kaon Physics in the 1990's: Rare Decays and CP Violation," in Proceedings of the 1988 Summer Study of High-Energy Physics in the 1990's, Snowmass, Colorado, June 27-July 15, 1988, S. Jensen, Ed. (World Scientific, Singapore, 1989), p. 370.

M. S. Atiya, I.-H. Chiang, J. S. Frank, J. S. Haggerty, M. M. Ito, T. F. Kycia, K. K. Li, L. S. Littenberg, A. Stevens, R. C. Strand, W. C. Louis, D. S. Akerib, M. Hildreth, D. R. Marlow, P. D. Meyers, M. A. Selen, F. C. Shormaker, A. J. S. Smith, G. Azuelos, E. W. Blackmore, D. A. Bryman, L. Felawka, P. Kitching, Y. Kuno, and J. A. MacDonald, "Search for a Light Higgs Boson in the Decay $\mathrm{K}^{+} \rightarrow \pi^{+} \mathrm{H}, \mathrm{H} \rightarrow \mu^{+} \mu^{-}, "$ Phys. Rev. Lett. 63, 2177 (1989).

I. P. Auer, E. Colton, W. R. Ditzler, H. Halpern, D. Hill, R. C. Miller, H. Spinka, N. Tamura, J.-J. Tavernier, G. Theodosiou, K. Toshioka, D. Underwood, R. Wagner, and A. Yokosawa, "Observation of Structures in the Mass Range of 2700 to $2900 \mathrm{MeV}$ in the Difference between the $p p$ Total Cross Sections for Pure Helicity States," Phys. Rev. Lett. 62, 2649 (1989).

F. T. Baker, L. Bimbot, B. Castel, R. W. Fergerson, C. Glashausser, A. Green, O. Hausser, K. Kicks, K. W. Jones, C. A. Miller, S. K. Nanda, R. D. Smith, M. Vetterli, J. Wamback, R. Abegg, D. Beatty, V. Cupps, C. Djalali, R. Henderson, K. P. Jackson, R. Jeppesen, J. Lisantti, M. Morlet, R. Sawafta, W. Unkelback, A. Willis, and S. Yen, "The Nuclear Spin Response to Intermediate Energy Protons," Phys. Lett. B 237, 337 (1990).

F. T. Baker, L. Bimbot, R. W. Fergerson, C. Glashausser, A. Green, K. W. Jones, W. G. Love, and S. Nanda, "Spin Dipole and Quadrupole Resonances in ${ }^{40} \mathrm{Ca}$," Phys. Rev. C 40, 1877 (1989).

M. L. Barlett, G. W. Hoffmann, L. Ray, G. Pauletta, K. H. McNaughton, J. F. Amann, K. W. Jones, J. B. McClelland, M. W. McNaughton, R. Fergerson, and D. Lopiano, "Inclusive and Exclusive Quasielastic $\vec{p}+{ }^{2} \mathrm{H}$ Spin Observables at 647 and $800 \mathrm{MeV}, "$ Phys. Rev. C 40, 2697 (1989). 
D. B. Barlow, B. M. K. Nefkens, C. T. Pillai, and K. W. Jones, "Production of High Momentum Negative Pions by $800-\mathrm{McV}$ Protons at $0^{\circ}$ to $20^{\circ}, " \mathrm{Nucl}$. Instrum. and Methods A 271, 471 (1988).

D. B. Barlow, R. S. Kessler, G. J. Kim, B. M. K. Nefkens, C. T. Pillai, J. W. Price, J. A. Wightman, S. L. Hall, D. W. Lane, S. R. Loe, L. K. Morton, M. E. Sadler, S. D. Adrian, W. J. Briscoe, L. H. Kramer, A. Mokhtari, A. M. Petrov, C. J. Seftor, M. F. Taragin, J. F. Davis, and I. Supck, 1."Measurement of Spin Rotation Parameters $A$ and $R$ for $\pi^{+} \pi$ and $\pi^{-} \pi$ Elastic Scattering from 471 to $625 \mathrm{McV} / c^{\prime \prime}$ "Phys. Rev. Lett. 62, 1009 (1989).

D. Berley, C. Y. Chang, B. L. Dingus, J. A. Goodman, T. J. Haines, M. Stark, R. W. Ellsworth, R. L. Burman, D. R. Cady, C. M. Hoffman, J. Lloyd-Evans, D. E. Nagle, M. E. Potter, V. D. Sandberg, C. A. Wilkinson, D. E. Alexandreas, R. C. Allen, S. Biller, G. Dion, X-Q. Lu, P. R. Vishwanath, and G. B. Yodh, "Status of the Expansion of the Cygnus Array at Los Alamos," in Proceedings of the XXI International Cosmic Ray Conference, Adelaide, Australia, January 6-19, 1990, R. J. Protheroe, Ed. (University of Adelaide, Physics Publications, 1989), Code HE 7.3-12, Vol. 10, p. 301.

E. Bjorklund, "Algorithms for Optimally Distributed Timing Pulses," Los Alamos National Laboratory document LA-UR-89-3558 (submitted to the Proceedings of the International Conference on Accelerator and Large Experimental Physics Control Systems, Vancouver, British Columbia, Canada, October 30-November 3, 1989).

E. Bjorklund and S. C. Schaller, "Data Archiving and Visualization Tool," Los Alamos National Laboratory document LA-UR-89-3111 (presented as part of the Los Alamos Accelerator Automation Toolkit Workshop, Los Alamos, New Mexico, October 31-Novembei 4, 1988).

R. L. Boudrie, " $(n, p)$ Experiments and Spectrometers," invited talk presented at the Long-Range Planning Meeting at Indiana University Cyclotron Facility, Bloomington, Indiana, June 9-11, 1989.

C. D. Bowman, J. D. Bowman, and V. W. Yuan, "Parity Violation in the 0.734-eV Neutron Resonance in ${ }^{139}$ La," Phys. Rev. C 39, 1721 (1989).

J. D. Bowman, C. D. Bowman, J. N. Knudson, S. Penttilä, S. J. Seestrom, J. J. Szymanski, V. W. Yuan, C. R. Gould, D. G. Haase, C. E. Mitchell, N. R. Roberson, P. P. J. Delheij, H. Postma, and E. D. Davis, "Search for Parity and Time Reversal Symmetry Violation Using Polarized Neutron-Nucleus Interactions," Los Alamos National Laboratory document LA-UR-89-3648 (submitted to the Proceedings of Fundamental Symmetries in Nuclei and Particles, International Symposium, Pasadena, California, September 7-9, 1989). 
W. H. Breunlich, P. Kammel, J. S. Cohen, and M. Leon, "Muon-Catalyzed Fusion," Ann. Rev. Nucl. Part. Sci. 39, 311 (1989).

M. A. Bryan, C. L. Morris, and G. C. Idzorek, "Actively-Stabilized Photomultiplier Tube Base for Vacuum Operation," Los Alamos National Laboratory report LA-11623-MS (May 1989).

T. Burchell, H. Frost, and W. F. Sommer, "New Instrumentation, Methodologies, Materials, and Facilities for Studies of Radiation Damage in Ceramics and Graphites," Los Alamos National Laboratory document LA-UR-89-4096 (submitted to the Japan-U.S. Cooperative Planning Meeting P-149, Tokyo, Japan, Decembet 12-13, 1989).

G. R. Burleson and K. J. Ernst, Eds., "The User's View for the Future of LAMPF, 1989: Reports from the Pion Physics Working Group," Los Alamos National Laboratory report LA-11673-MS (December 1989).

Michael Burlein, "Pion Inelastic Scattering from ${ }^{20}$ Ne," Ph.D. thesis, University of Pennsylvania, Los Alamos National Laboratory report LA-11697-T (December 1989).

R. L. Burman, C. M. Hoffman, D. E. Nagle, M. E. Potter, V. D. Sandberg, D. Berley, B. L. Dingus, J. A. Goodman, T. J. Haines, M. Stark, R. W. Ellsworth, D. E. Alexandreas, R. C. Allen, S. Biller, G. Dion, X-Q. Lu, P. R. Vishwanath, G. B. Yodh, T. Tumer, S. White, A. Zych, S. Freedman, D. Krakauer, and R. Lamb, "A Low-Threshold EAS Array for Gamma-Ray Astronomy at Los Alamos," in Proceedings of the XXI International Cosmic Ray Conference, Adelaide, Australia, January 6-19, 1990, R. J. Protheroe, Ed. (University of Adelaide, Physics Publications, September 1989), Code HE 7.3-11, Vol. 10, p. 297.

R. L. Burman, M. E. Potter, and E. S. Smith, "Monte Carlo Simulation of Neutrino Production by Medium-Energy Protons in a Beam Stop," Nucl. Instrum. and Methods A 291, 621 (1990).

M. Burns, "Determination of Beam Position and Angle to Minimize Beam Loss in the Proton Storage Ring," Los Alamos National Laboratory document LA-UR-893623 (submitted to the Proceedings of the International Conference on Accelerator and Large Experimental Physics Control Systems, Vancouver, British Columbia, Canada, October 30-November 3, 1989).

H. S. Butler, "Control System Considerations for the AHF," Los Alamos National Laboiatory document LA-UR-89-1231 (submitted to the Proceedings of the Accelerator Design Workshop, Los Alamos National Laboratory, February 20-25, 1989). 
H. S. Butler, "Coping with Large Sextupole Errors in Bending Magnets," Los Alamos National Laboratory document LA-UR-89-1232 (submitted to the Proceedings of the Accelerator Design Workshop, Los Alamos National Laboratory, February $20-25,1989$ ).

T. Callaway, "Accelerator Control Consoles: An Operators Perspective," Los Alamos National Laboratory document LA-UR-89-3631 (submitted to the Proceedings of the International Conference on Accelerator and Large Experimental Physics Control Systems, Vancouver, British Columbia, Canada, October 30-November 3, 1989).

D. C. Carey, R. N. Coleman, M. D. Corcoran, J. D. Cossairt, A. A. Derevschikov, D. P. Grosnick, D. Hill, K. Imai, A. Konaka, K. Kuroda, F. Lehar, A. de Lesquen, D. Lopiano, F. C. Luehring, T. Maki, S. Makino, A. Masaike, Yu. A. Matulenko, A. P. Meschanin, A. Michalowicz, D. H. Miller, K. Miyake, T. Nagamine, T. Nakano, F. Nessi-Tedaldi, M. Nessi, C. Nguyen, S. B. Nurushev, Y. Ohashi, G. Pauletta, A. Penzo, G. C. Phillips, A. L. Read, J. B. Roberts, L. van Rossum, G. Salvato, P. Schiavon, T. Shima, V. L. Solovyanov, H. Spinka, R. W. Stanek, R. Takashima, F. Takeutchi, N. Tamura, N. Tanaka, D. G. Underwood, A. N. Vasiliev, A. Villari, J. L. White, A. Yokosawa, T. Yoshida, and A. Zanetti, "Measurement of the Analyzing-Power in the Primakoff Process with a High-Energy Polarized-Proton Beam," Phys. Rev. Lett. 64, 3576 (1990).

S. Carpenter, M. N. Cornforth, W. F. Harvey, M. R. Raju, M. E. Schillaci, M. E. Wilder, and D. T. Goodhead, "Radiobiology of Ultrasoft X Rays: IV. Flat and Round Shaped Hamster Cells (CHO-10B, HS-23)," Radiat. Res. 119, 523 (1989).

G. P. Carr, "Emulating Conventional Operator Interfaces on Window-Based Workstations," Los Alamos National Laboratory document LA-UR-89-3560 (submitted to the Proceedings of the International Conference on Accelerator and Large Experimental Physics Control Systems, Vancouver, British Columbia, Canada, October 30-November 3, 1989).

R. E. Chrien, S. Bart, M. May, P. H. Pile, R. J. Sutter, R. Hackenburg, P. Barnes, R. Rieder, J. Seydoux, B. Bassaleck, R. Eisenstein, R. Grace, W. Wharton, D. Marlow, J. Derderian, Y. Civelkekoglu, M. Deutsch, J. Prater, C. Chu, E. Hungerford, T. Kishimoto, T. Fukuda, M. Barlett, G. Hoffman, E. C. Milner, and R. L. Stearns, "Search for Radiative Transitions in the Hypernucleus $\lambda^{10} \mathrm{~B}$, " Los Alamos National Laboratory document LA-UR-89-3100 (submitted to Physical Review C, August 1989).

Douglas Ciskowski, "Cross Sections and Analyzing Powers of ${ }^{15} \mathrm{~N}(p, n)^{15} \mathrm{O}$ at $200 \mathrm{MeV}$ and $494 \mathrm{MeV}$," Ph.D. thesis, University of Texas at Austin, Los Alamos National Laboratory report LA-11685-T (November 1989). 
B. L. Clausen, J. T. Brack, M. R. Braunstein, J. J. Kraushaar, R. A. Loveman, R. J. Peterson, R. A. Ristinen, R. A. Lindgren, and M. A. Plum, "Analysis of Pion Scattering to Stretched States in ${ }^{69} \mathrm{Ni}$ and Other Nuclei Using Woods-Saxon Wave Functions," Phys. Rev. C41, 2246 (1990).

M. Clay, "What Makes a Control System Usable? An Operational Viewpoint," Los Alamos National Laboratory document LA-LIR-89-3574 (submilted to the Proceedings of the International Conference on Accelerator and Large. Experimental Physics Control Systems, Vancouver, British Columbia, Canada, October 30-November 3, 1989).

Stanley Cohen, "The Development of a Standard File Format for Accelerator Data Analysis," Los Alamos National Laboratory document LA-UR-89-3596 (submitted to the Proceedings of the International International Conference on Accelerator and Large Experimental Physics Control Systems, Vancouver, British Columbia, Canada, October 30-November 3, 1989).

R. Coleman, B. Winstein, G. Bock, M. D. Cooer, J. Enagonio, A. Erwin, J. Fry, H. Greenlee, B. Hsiung, K. McFarlane, W. Morse, T. Shinkawa, G. Thomson, B. Tschirhart, Y. Wah, H. Yamamoto, and T. Yamanaka, "Symmetry Violations and Rare Decays," Los Alamos National Laboratory document LA-UR-90-158 (submitted to the Pr ceedings of Fermilab: Physics in the 1990's, Batavia, Illinois, August 16-24, 1989).

E. P. Colton, "New Compressor at Los Alamos," in Proceedings of the 1988 Summer Study on :Figh-Energy Physics in the 1990's, Snowmass, Colorado, June 27July 15, 1988, S. Jensen, Ed. (World Scientific, Singapore, 1989), ISBN 9971-50$841-9$, p. 445.

E. P. Colton, "Study of the Barrier Bucket in PSR," Los Alamos National Laboratory document AHF Technical Note 88-024 (November 1988).

E. P. Colton, "Design for a Second-Generation Proton Storage Ring at LAMPF," Los Alamos National Laboratory document LA-UR-88-4033 (submitted to the Proceedings of ICANS-X, Los Alamos National Laboratory, October 3-7, 1988).

E. P. Colton, "Maximum Beam in PSR-1988," Los Alamos National Laboratory document AHF Technical Note 88-026 (November 1988).

E. P. Colton, "Debunching Experiments in PSR," Los Alamos National Laboratory document AHF Technical Note 88-027 (December 1988). 
E. P. Colton, "Strawman 1.6-GeV Compressor Design," in Proceedings of the Accelerator Design Workshop, February 22-27, 1988, Los Alamos, New Mexico, Los Alamos National Laboratory report LA-11432-C (1989).

E. P. Colton and H. A. Thiessen, " $\mathrm{H}$ - Injection into the Low-Energy Booster of the SSC," in Proceedings of the 1988 Summer Study on High-Energy Physics in the 1990's, Snowmass, Colorado, June 27-July 15, 1988, S. Jensen, Ed. (World Scientific, Singapore, 1989), ISBN 9971-50-841-9, pp. 542-545.

J. S. Conway, C. E. Adolphsen, J. P. Alexander, K. J. Anderson, J. G. Heinrich, J. E. Pilcher, A. Possoz, E. I. Rosenberg, C. Biino, J. F. Greenhalgh, W. C. Louis, K. T. McDonald, S. Palestini, F. C. Shoemaker, and A. J. S. Smith, "Experimental Study of Muon Pairs Produced by 252-GeV Pions on Tungsten," Phys. Rev. D 39, 92 (1989).

D. W. Cooke, M. S. Jahan, J. L. Smith, M. A. Maez, W. L. Hults, I. D. Raistrick, D. E. Petarson, J. A. O'Rourke, S. A. Richardson, J. D. Doss, E. R. Gray, B. Rusnak, G. P. Lawrence, and C. Fortgang, "Detection of Surface $(\sim 1 \mu \mathrm{m})$ Impurity Phases in High $T_{c}$ Superconductors," Appl. Phys. Lett. 54, 960 (1989).

D. W. Cooke, R. Hutson, R. Kwok, M. Macz, H. Rempp, M. Schillaci, J. Smith, J. Willis, R. Lichti, K. Chan, C. Boekema, S. Weathersby, and J. Oostens, "Muon Spin Rotation Measurement of Magnetic Field Penetration and Flux Pinning in Superconducting $\mathrm{EuBa}_{2} \mathrm{Cu}_{3} \mathrm{O}_{x},{ }^{\prime \prime}$ Phys. Rev. B 39, 2748 (1989).

D. W. Cooke, "Charge Trapping and Luminescence in Biochemical Systems," Los Alamos National Laboratory document LA-UR-88-2488 (submitted to International Journal of Radiation Applications and Instrumentation, Part C: Radiation Physics and Chemistry, July 1988).

D. W. Cooke, M. T. Trkula, R. E. Muenchausen, N. S. Nogar, and J. L. Smith, "Characterization of High-Temperature Superconductors by Optical Techniques," Los Alamos National Laboratory document LA-UR-88-3760 (submitted to Journal of the Optical Society of America B, November 1988).

D. W. Cooke, E. R. Gray, R. J. Houlton, B. Rusnak, E. Meyer, G. P. Lawrence, M. A. Maez, B. Bennett, J. D. Doss, A. Mayer, W. L. Hults, and J. L. Smith, "RF Surface Resistance of Bulk $\mathrm{TI}_{2} \mathrm{Ca}_{2} \mathrm{Ba}_{2} \mathrm{Cu}_{3} \mathrm{O}_{10}, \mathrm{Bi}_{2} \mathrm{CaSr}_{2} \mathrm{Cu}_{2} \mathrm{O}_{8}$, and $\mathrm{YBa}_{2} \mathrm{Cu}_{3} \mathrm{O}_{x}$," Los Alamos National Laboratory document LA-UR-88-4283 (submitted to Physical Review B, December 1988).

D. W. Cooke, B. Bennett, E. R. Gray, R. J. Houlton, W. L. Hults, M. A. Maez, A. Mayer, and J. L. Smith, "Correlation of Thermally Stimulated Luminescence with Radio Frequency Surface Resistance," Appl. Phys. Lett. 55, 1038 (1989). 
D. W. Cooke, E. R. Gray, R. J. Houlton, B. Rusnak, E. A. Meyer, J. G. Berry, D. R. Brown, F. H. Garzon, I. D. Raistrick, A. D. Rollet, and R. Bolmaro, "Surface Resistance of $\mathrm{YBa}_{2} \mathrm{Cu}_{3} \mathrm{O}_{7}$ Films on $\mathrm{SrTiO}_{3}$ and $\mathrm{LaGaO}_{3}$ Substrates," Appl. Phys. Lett. 55, 914 (1989).

D. W. Cooke and E. R. Gray, "High-Frequency Cavity Applications and Measurements of High-Temperature Superconductors," Los Alamos National Laboratory document LA-UR-89-1852 (submitted to the Proceedings of the Conference on High Temperature Superconductivity, Huntsville, Alabama, May 23-25, 1989).

D. W. Cooke, E. R. Gray, H. H. S. Javadi, R. J. Houlton, B. Rusnak, E. A. Meyer, P. N. Arendt, N. Klein, G. Müller, S. Orbach, H. Piel, L. Drabeck, G. Grüner, J. Y. Josefowicz, D. B. Rensch, and F. Krajenbrink, "Frequency Dependence of the Surface Resistance in High-Temperature Superconductors," Los Alamos National Laboratory document LA-UR-89-2245 (submitted to Solid State Communications, July 1989).

D. W. Cooke, R. S. Kwok, R. L. Lichti, T. R. Adams, C. Boekenia: W. K. Dawson, A. Kebede; J. Schwegler, J. E. Crow, and T. Mihalisin, "Magnetic Ordering in $\left(\mathrm{Y}_{1-x} \operatorname{Pr}_{x}\right) \mathrm{Ba}_{2} \mathrm{Cu}_{3} \mathrm{O}_{7}$ as Observed by Muon Spin Relaxation," Los Alamos National Laboratory document LA-UR-89-2698 (submitted to Physical Review Letters, August 1989).

D. W. Cooke, E. R. Gray, R. J. Houlton, H. H. S. Javadi, M. A. Maez, B. L. Bennett, B. Rusnak, E. A. Meyer, P. N. Arendt, J. G. Beery, D. R. Brown, F. H. Garzon, I. D. Raistrick, A. D. Rollett, B. Bolmaro, N. E. Elliott, N. Klein, G. Müller, S. Orbach, H. Piel, J. Y. Josefowicz, D. B. Rensch, L. Drabeck, and G. Grüner, "Surface Resistance of $\mathrm{YBa}_{2} \mathrm{Cu}_{3} \mathrm{O}_{7}$ Films Deposited on $\mathrm{LaGaO}_{3}$ Substrates," Los Alamos National Laboratory document LA-UR-89-2699 (submitted to the Proceedings of the International $M^{2} S-H T S C$ Conference (Physica C), Stanford, California, July 2328, 1989).

D. W. Cooke, R. S. Kwok, M. S. Jahan, R. L. Lichti, T. R. Adams, C. Boekema, W. K. Dawson, A. Kebede, J. Schwegler, J. E. Crow, and T. Mihalisin, " $\mu$ SR Investigation of the Coexistence of Magnetism and Superconductivity in $\left(\mathrm{Y}_{1-x} \mathrm{Pr}_{x}\right) \mathrm{Ba}_{2} \mathrm{Cu}_{3} \mathrm{O}_{7}$," Los Alamos National Laboratory document LA-UR-893221 (submitted to the Proceedings of the International Conference on Physics of Highly Correlated Electrons, Santa Fe, New Mexico, September 11-15, 1989).

D. W. Cooke, E. R. Gray, R. J. Houlton, G. P. Lawrence, J. A. McGill, E. Meyer, C. Morris, B. Rusnak, and T. P. Starke, "RF Superconductivity Research and Development at Los Alamos National Laboratory," Los Alamos National Laboratory document LA-UR-89-3331 (submitted to the Proceedings of The 4th Workshop on RF Superconductivity, Tsukuba, Japan, August 14-18, 1989). 
D. W. Cooke, R. S. Kwok, M. S. Jahan, R. L. Lichti, T. D. Adams, C. Boekema, W. D. Dawson, A. Kebede, J. Schwegler, J. E. Crow, and T. Mihalisin, "Magnetic Ordering in $\left(\mathrm{Y}_{1-x} \mathrm{Pr}_{x}\right) \mathrm{Ba}_{2} \mathrm{Cu}_{3} \mathrm{O}_{7}$ as Evidenced by Muon Spin Relaxation," Los Alamos National Laboratory document LA-UR-89-3380 (submitted to the Proceedings of the 34th Annual Conference on Magnetism and Magnetic Materials, Boston, Massachusetts, November 28-December 1, 1989).

M. N. Cornforth, M. E. Schillaci, D. T. Goodhead, S. G. Carpenter, M. E. Wilder, R. J. Sebring, and M. R. Raju, "Radiobiology of Ultrasoft X Rays: IIl. Normal Human Fibrob' asts and the Significance of Terminal Tract Structure in Cell Inactivation," Radiat. Res. 119, 511 (1989).

B. L. Dingus, C. Y. Chang, J. A. Goodman, S. K. Gupta, T. J. Haines, D. A. Krakauer, R. L. Talaga, R. L. Burman, K. B. Butterfield, R. Cady, J. Lloyd-Evans, D. E. Nagle, M. E. Potter, V. D. Sandberg, C. A. Wilkinson, D. E. Alexandreas, R. C. Allen, and G. B. Yodh, "Observation of HERCULES X-1 at Energies Above $50 \mathrm{TeV}, "$ in Proceedings of the American Physical Soriety Division of Particles and Fields Meeting, Storrs, Connecticut, August 15-18, 1988, K. Haller, D. G. Galdi, M. M. Islam, R. L. Mallett, P. D. Mannheim, and M. S. Swanson, Eds. (World Scientific, Singapore, 1989), pp. 252-254.

J. D. Doss, D. W. Cooke, P. N. Arendt, M. Nastasi, R. E Muenchausen, and J. R. Tesmer, "Instrument for Characterization of Superconducting Films," Supercond. Sci. Technol. 2, 63 (1989).

L. S. Durkin, J. S. Fitch, R. W. Harper, J. W. Heimaster, T. Y. Ling, J. W. Mitchel, T. A. Romanowski, C. J. Ruish, V. K. Sehgal, E. S. Smith, M. Timko, B. K. Fujikawa, R. McKcown, W. C. Choi, A. Fazely, R. L. Imlay, W. J. Metcalf, W. Marterer, J. B. Donahue, and V. Sandberg, "A Fine-Grain Modular Detector for Low-Energy Particles," Nucl. Instrum. and Methods A 277, 386 (1989).

R. Garfagnini, G. Pauletta, L. Santa, N.. Barlett, D. Ciskowski. G. W. Hoffmann, G. Pauletta, M. M. Gazzaly, K. W. Jcnes, N. Tanaka, M. A. Nasser, C. Smith, and R. Whitney, "Evidence for Narrow Structure in the Analyzing Power of the ${ }^{3} \mathrm{He}(\vec{p}, d) \mathrm{X}$ Reaction at $0.8 \mathrm{GeV}, "$ invited talk presented by $\mathrm{N}$. Tanaka at the Leningrad Nuclear Physics Institute, Leningrad, U'SSR, on September 15, 1989, Los Alamos National Laboratory document LA-UR-89-2580 (1989).

S. Gottesfeld, R. E. Anderson, D. A. Baker, R. D. Bolton, K. B. Butterfield, F. H. Garzon, C. A. Goulding, M. W. Johnson, E. M. Leonard, T. L. Springer, and T. Zawodzinski, "Experiments and Nuclear Measurements in Search of Cold Fusion Processes," Los Alamos National Laboratory document LA-UR-89-3711 (submitted to the Froceedings of the Workshop on Cold rusion, Santa Fe, New Mexico, May 23-25, 1989, and to be published in the journal of Fusion Energy, 1989). 
P. A. M. Gram, S. A. Wood, S. Hфibräten, E. R. Kinney, P. Mansky, J. J. Matthews, T. Soos, G. A. Rebka, Jr., and D. A. Roberts, "Dependenca of the Cross Section for Inclusive Pion Double Charge Exchange on Nuclear Mass and Charge," Phys. Rev. Lett. 62, 1837 (1989).

S. J. Green. "Atom Smashers," presented for Science Beginnings at the Lab ratory program, Los Alamos, New Mexico (March 1989).

S. J. Greene, "Aspects of Nuclear Physics," presented for High School Cooperative program, Los Alamos, New Mexico (December 1989).

D. P. Grosnick, D. A. Hill, M. R. Laghai, D. Lopiano, Y. Ohashi, T. Shima, H. Spinka, R. W. Stanek, D. G. Underwood, A. Yokosawa, F. Lehar, A. de Lesquen, L. van Rossum, D. C. Carey, R. N. Coleman, J. D. Cossairt, A. L. Read, R. Schailey, A. A. Derevschikov, Yu. A. Matulenko, A. P. Meschanin, S. B. Nurushev, R. A. Rzaev, V. L. Solovyanov, A. N. Vasiliev, N. Akchurin, Y. Onel, K. Imai, S. Makino, A. Masaike, K. Miyake, T. Nagamine, N. Tamura, T. Yoshida, R. Takashima, F. Takeuichi, A. Maki, K. Kuroda, A. Michalowicz, G. Shapiro, N. Tanaka, G. Salvato, A. Villari, M. Gazzaly, F. C. Luehring, D. H. Miller, M. D. Corcoran, F. Nessi-Tedaldi, M. Nessi, C. Nguyen, G. C. Phillips, J. B. Roberts, J. L. White, G. Pauletta, R. Birsa, F. Bradamante, S. Dalla Torre-Colautti, M. Gtorgi, A. Martin, A. Penzo, P. Schiavon, and A. Zanetti, "The Design and Performance of the FNAL High-Energy Polarized-Beam Facility," Nucl. Instrum. and Methods A 290, 269 (1990).

G. Guignard, "A Lattice With No Transition and Large Dynamic Aperture," Los Alamos National Laboratory document LA-UR-89-767 (submitted to the Proceedings of the 1989 Particle Accelerator Conference, Chicago, Illinois, March 20-23, 1989).

G. Guignard, "Main Ring Lattice and Beam Dynamics," Los Alamos National Laboratory document LA-UR-89-1225 (submitted to the Proceedings of the Accelerator Design Workshop, Los Alamos National Laboratory, February 20-25, 1989).

T. J. Haines, D. Berley, C. Y. Chang, B. L. Dingus, J. A. Goodman, M. Stark, R. L. Burman, D. R. Cady, C. M. Hoffman, J. Lloyd-Evans, D. E. Nagle, M. E. Potter, V. D. Sandberg, C. A. Wilkinson, D. E. Alexandreas, R. C. Allen, S. Biller, G. M. Dion, P. R. Vishwanath, G. B. Yodh, and R. W. Ellsworth, "The Status of the CYGNUS Experiment: Past, Present, and Future," Los Alamos National Lahoratory document LA-UR-89-2482 (submitted to the Proceedings of the Workshop on Physics and Experimental Techniques of High Energy Neutrinos and VHE and LHE Gamma-Ray Particle Astrophysics, Little Rock, Arkansas, May 11-13, 1989). 
T. J. Haines, D. E. Alexandreas, R. C. Allen, S. Biller, D. Berley, R. L. Burman, D. k. Cady. C. Y. Chang, B. L. Dingus, G. Dion, R. W. Ellsworth, J. A. Goodman, C. M. Hoffman, J. Lloyd-Evans, D. E. Nagle, M. Potter, V. D. Sandberg, C. A. Wilkinson, and G. B. Yodh, "A Limit on Possible Energy-Dependent Velocities for Massless Particles," Phys. Rev. D 41, 69? (1990).

B. M. Han, G. G. Karady, and H. A. Thiessen, "Tuning Magnet Power Supply," Los Alamos National Laboratory document LA-UR-89-1572 (submitted to the Proceedings of the 1989 Particle Accelerator Conference, Chicago, Illinois, March 2023, 1989).

P. G. Harris, H. C. Bryant, A. H. Mohagheghi, C. Y. Tang, J. B. Donahue, C. R. Quick, R. A. Reeder, Stanley Cohen, W. W. Smith, J. E. Stewart, "Further Studies of the Photodetachment Cross Section of the $\mathrm{H}^{-}$Ion in Electric Fields," Los Alamos National Laboratory document LA-UR-89-322 (1989).

P. G. Harris, H. C. Bryant, A. H. Mohagheghi, R. A. Reeder, C. Y. Tang, J. B. Donahue, C. R. Quick, D. Rislov, H. Sarifian, W. W. Smith, H. Toutounchi, and T. C. Altman, "Measurement and Reduction of Momentum Spread in the LAMPF Linac Beam," Los Alamos National Laboratory document LA-UR-89-3692 (to be rublished in Nuclear Instruments and Methods, 1989).

R. J. Hayden, M. J. Jakobson, O. B. van Dyck, and R. L. York, "Beam Dynamics Calculations for the LAMPF Optically Pumped Ion source, " Los Alamos National Laboratory document LA-UR-89-1159 (submitted to the Proceedings of the Particle Accelerator Conference, March 1989).

R. H. Heffner, D. W. Cooke, A. L. Giorgi, R. I. Hutson, M. E. Schillaci, H. D. Rempp, J. L. Smith, J. O. Willis, D. E. MacLaughlin, C. Boekema, R. L. Lichti, J. Oostens, and A. B. Denison, "Muon Spin Rotation in the Magnetic and Superconducting Ground States of (U,'Th)Be $\mathrm{B}_{13}$ and (U,Th)Pt ${ }_{3}$," Phys. Rev. B 39, 11345 (1989).

J. G. Heinrich, C. E. Adolphsen, J. P. Alexander, K. J. Anderson, J. S. Conway, J. E. Pilcher, A. Possz, E. I. Rosenberg, C. Biino, J. F. Greenhalgh, W. C. Louis, K. T. McDonald, S. Palestini, F. C. Shocmaker, and A. J. S. Smith, "Measurement of the Ratio of Sea to Vale ce Quarks in the Nucleon," Phys. Rev. Lett. 63, 356 (1989).

G. W. Hoffmann, M. L. Barlett, D. Ciskowski, G. Pauletta, M. Purcell, L. Ray, J. F. Amunn, J. J. Jarmer, K. W. Jones, S. Penttilä, N. Tanaka, M. M. Gazzaly, J. R. Comfort, B. C. Clark, and S. Hame, "Cross Sections, Analyzing Powers, and Spin-Rotation-Depolarization Obser, thl ss for 500-MeV Proton Elastic Scattering 
from ${ }^{12} \mathrm{C}$ and ${ }^{13} \mathrm{C}$, , Los Alamos National Laboratory document LA-UR-89-3793 (submitted to Physics Letters B, October 1989).

S. Hфibräten, "Coincidence Measurement of the $\left(\pi^{+} \pi^{0} p\right)$ Reaction in the $\Delta$ Resonance Region," Ph.D. thesis, Massachusetts Institute of Technology, 1989, Los Alamos National Laboratory report LA-11582-T (February 1989).

D. J. Horen, J. Lisantti, R. L. Auble, F. E. Bertrand, B. L. Burks, E. E. Gross, R. O. Sayer, D. K. McDaniels, K. W. Jones, J. B. McClelland, S. J. Seestrom-Morris, and L. W. Swenson, " $T=1$, Spin-Dipole Strength in ${ }^{4 n} \mathrm{Ca}$," Z. Phys. A 333, 39 (1989).

J. W. Hurd and H. A. Thiessen, "Spectrometer Chopper for AHF and SSC LowEnergy Transports," Los Alamos National Laboratory document LA-UR-89-840 (submitted to the 1989 Particle Accelerator Conference, Chicago, Illinois, March 2023, 1989).

M. S. Jahan, D. W. Cooke, H. Sheinberg, J. L. Smith, and D. P. Lianos, "Environmental Effects on Luminescence Yield of Superconducting $\mathrm{YBa}_{2} \mathrm{Cu}_{3} \mathrm{O}_{x}$," J. Mat. Res. 4, 759 (1989).

K. W. Jones, in "Spin Physics at LAMPF," in Proceedings of the LAMPF Annual User's Meeting, Los Alamos, New Mexico, October 17-19, 1988, Los Alamos National Laboratory report LA-11556-C (April 1989).

G. G. Karady and H. A. Thiessen, "Optimization of Resonant Power Supply Circuit," Los Alamos National document LA-UR-89-1571 (submitted to the Proceedings of the 1989 Particle Accelerator Conference, Chicago, Illinois, March 20-23, 1989).

G. G. Karady and H. A. Thiessen, "New Energy Replacement Method of Resonant Power Supplies," Los Alamos National document LA-UR-89-1573 (submitted to the Proceedings of the 1989 Particle Accelerator Conference, Chicago, Illinois, March 20 23, 1989).

C. J. Kenncy, M. Eckhause, J. F. Ginkel, P. P. Guss, J. R. Kane, W. F. Vulcan, R. E. Welsh, R. J. Whyley, J. Bilskie, G. W. Hart, W. W. Kinnison, D. M. Lee, R. j. McKee, Jr., E. C. Milner, G. H. Sanders, H.-J. Ziock, and D. Huang, "Use of Proportional Tubes in a Muon Polarimeter," Los Alamos National Laboratory document LA-UR-89-420 (submitted o the Proceedings of the IEEE 1988 Nuclear Sciences Symposium, Orlando, Florida, November 1988).

E. R. Kinney, "Inclusive Pion Double Charge Exchange in ${ }^{4} \mathrm{He}$ at Intermediate Energies," Ph.D. thesis, Massachusetts Institute of Technology, Los Alamos National Laboratory report LA-11417-T (October 1988). 
Y. Kuang, "Observation of the PNegative Muonium Ion in Vacuum," Ph.D. thesis, Yale University, Los Alamos National Laboratory report LA-11575-T (May 1989).

M. J. Leitch, H. W. Baer, R. L. Burman, C. L. Morris, J. N. Knudson, J. R. Comfort, D. H. Wright, R. Gilman, S. H. Rokni, E. Piasetzky, A. Weinfeld, W. R. Gibbs, and W. B. Kaufmann, "The ${ }^{14} \mathrm{C}\left(\pi^{+}, \pi^{-}\right)^{14} \mathrm{O}$ Reaction between 19 and $80 \mathrm{MeV}$," Phys. Rev. C 39, 2356 (1989).

M. Leon, "Three-body Molecular Formation in Muon-Catalyzed Fusion $d-\imath$ Fusion: The Role of Configuration Mixing," Fhys. Rev. A 38, 5554 (1989).

M. Leon, "Interpretation of Recent LAMPF Results on $d t \mu$ Formation," Los Alamos National Laboratory document LA-UR-89-3535 (submitted to Proceedings of the $\mu$ CF Workshop, Oxford, United Kingdom, September 1989).

Z. Li and H. A. Thiessen, "Kicker Magnet Design," Los Alamos National Laboratory document Ls.-UR-89-1257 (submitted to the Proceedings of the Accelerator Design Workshop, Los Alamos, New Mexico, February 20-25, 1989).

R. L. Lichti, K. C. B. Chan, D. W. Cooke, and C. Bockema, "Coupling Strengths and Flux Pinning in Oxide Superconductors," Appl. Phys. Lett. 54, 2361 (1989).

J. Lisantti, F. E. Bertrand, D. J. Horen, R. L. Auble, B. L. Burks, E. E. Gross, R. O. Sayer, D. K. McDaniels, K. W. Jones, J. B. McClelland, S. J. Seestrom-Morris, and L. W. Swenson, "Collective Model DWBA Analysis of 500-MeV Proton Scattering from ${ }^{41} \mathrm{Ca}$, " Phys. Rev. C 39, 568 (1989).

J. Lisantti, D. J. Horen, F. E. Bertrand, R. L. Auble, B. L. Burks, E. E. Gross, R. O. Sayer, D. K. McDaniels, K. W. Jones, J. B. McClelland, S. J. Seestrom-Morris, and L. W. Swenson, "Giant Resonance Strength Distribution in ${ }^{40} \mathrm{Ca}$ from Inciastic Scattering of 500-MeV Protons," Phys. Rtv. C40, 211 (1989).

X.-Q. Lu, G. Yodh, S. Y. Fung, J. H. Kang, B. C. Shen, G. J. VanDalen, R. C. Carlini, J. J. Napolitano, J. Amann, H. Bacr, R. Burman, J. Donahue, IN. Foreman, G. T. Garvey, M. Hoehn, T. Kozlowski, D. M. Lee, W. C. Louis, J. 'McClelland, E. C. Milner, M. Oothoudt, V. Sandberg, M. Schillaci, N. Tanaka, R. Werbeck, D. H. White, D. Whitehouse, A. Fazely, M. Brooks, B. B. Dieterle, C. P. Leavitt, R. Reeder, A. K. Mann, and W. K. McFarlane, "Proposal to Sea ch for Neutrino Oscillations with High Sensitivity in the Appearance Channels $\nu_{\mu} \rightarrow \nu_{e}$ and $\bar{\nu}_{\mu}-\bar{\nu}_{e}, "$ Los Alamos National Laboratory document LA-UR-89-3764, LAMPF Research Proposal (December 1989).

C. Mathiazhagan, W. R. Molzon, R. D. Cousins, J. Konigsberg, J. Kubic, P. Melese, P. Rubin, W. E. Slater, D. Wagner, G. W. Hart, W. W. Kinnison, D. M. Lee, R. 

J. Urheim, S. Axelrod, K. A. Biery, G. M. Irwin, K. Lang, J. Margulies, D. A. Ouimette, J. L. Ritchie, Q. H. Trang, S. B. Wojcicki, L. B. Aucrbach, P. Buchholz, V. L. Highland, W. K. McFarlane, M. Sivertz, M. D. Chapman, M. Eckhause, J. F. Ginkel, A. D. Hancock, D. Joyce, J. R. Kane, C. J. Kenney, iN. F. Vulcan, R. E. Welsh, R. J. Whyley, and R. G. Winter, "New Experimental Limits on $K_{L}^{0} \rightarrow \mu e$ and $K_{L}^{0} \rightarrow e e$ Branching Ratios," Phys. Rev. Lett. 63, 2181 (1989).

C. Mathiazhagan, W. R. Molzon, R. D. Cousins, J. Konigsberg, J. Kubic, P. Melese, P. Rubin, W. E. Slater, D. Wagner, G. W. Hart, W. W. Kinnison, D. M. Lee, R. J. McKee, Jr., E. C. Milner, G. H. Sanders, H. J. Ziock, K. Arisaka, P. Knibbe, J. Urheim, S. Axelrod, K. A. Biery, G. M. Irwin, K. Lang, J. Margulies, D. A. Ouimette, J. L. Ritchie, Q. H. Trang, S. G. Wojcicki, L. B. Aucrbach, P. Buchholz, V. L. Highland, W. K. McFarlane, M. Eckhause, J. F. Ginkel, A. D. Hancock, D. Joyce, J. R. Kane, C. J. Kenney, W. F. Vulcan, R. E. Welsh, R. J. Whyley, and R. G. Winter, "Measurement of the Branching Ratio for the Decay $K_{L}^{0} \rightarrow \mu \mu$," Phys. Rev. Lett. 63, 2185 (1989).

W. K. McFarlane and M. D. Cooper, "Search for $K_{L}^{0} \rightarrow \mu e$ at the $10^{-13}$ Level," $_{\nu}$ in Proceedings of the 1988 Summer Study on High Energy Physics in the 1990's, Snowmass, Colorado, June 27-July 15, 1988, S. Jensen, Ed. (World Scientific, 1989), p. 357.

J. B. McClelland, D. A. Clark, J. L. Davis, R. C. Haight, R. W. Johnson, N. S. P. King, G. L. Morgan, L. \}. Rybarcyk, John Ullmann, Paul Lisowski, W. R. Smythe, D. A. Lind, C. D. Zafiratos, and J. Rapaport, "Longitudinal Linac Beam Focusing for Neutron Time-of-Flight Measurements," Nucl. Instrum. and Methods A276, 35 (1989).

Mike McNaughton, "Newton and Rockets, Einstein and Starships," Los Alamos National Laboratory document LA-UR-89-3187 (subinitted to The Physics Teacher and Fundamental Physics, November 1989).

M. M. Meier, W. B. Amian, D. A. Clark, C. A. Goulding, J. B. McClelland, G. L. Morgan, and C. E. Moss, "Differential Neutron Production Cross Sections and Neutron Yields from Stopping-Length Targets for 113-MeV Protons," Nucl. Sci. Eng. 102, 310 (1989); Los Alamos National Laboratory report LA-11518-MS (March 1989).

R. E. Mischke, "MEGA--An Experiment to Search for $\mu \rightarrow e \gamma$ at the Level of 10-13," Los Alamos National Laboratory document LA-UR-90-134 (submitted to the International Seminar on Intermediate Energy Physics (INES-89), Moscow, USSR, November 27-30, 1989). 
J. W. Mitchell, "Results of a Neutrino Oscillation Experiment Performed at a Meson Factory Beam-Stop," Ph.D. thesis, Ohio State University, Los Alamos National Laboratory report LA-11539-T (April 1989).

S. Mordechai, M. Burlein, H. T. Fortune, S. J. Greene, G. Liu, C. Fred Moore, C. L. Morris, J. M. O'Donnell, A. H. Wuosmaa, and S. H. Yoo, "Observation of Double Giant Dipole Resonances in Pion Double Charge Exchange," invited paper presenied by C. L. Morris at the 1989 International Nuclear Physics Conference, August 2-26, 1989, Sao Paulo, SP Brazil, Los Alamos National Laboratory document LA-UR-89-1288 (1989).

Christopher L. Morris, "Uses of Superconducting RF Structures in Pion Beams at LAMPF," invited talk presented at Applications in Research and Industry '88, Denton, Texas (November 1989).

Christopher L. Morris, "Spin Excitations with Pions at LAMPF," presented at the International Symposium on Heavy Ion Research with Magnetic Spectrographs, East Lansing, Michigan (January 1989).

Christopher L. Morris, "High-Resolution Position Sensitive Detectors," presented at the International Symposium on Heavy Ion Research with Magnetic Spectrographs, East Lansing, Michigan (January 1989).

Christopher L. Morris, "Future of DCX at LAMPF," presented at the Second International Workshop on Pion-Nucleus Double Charge Exchange, Los Alamos, New Mexico (August 1989).

Christopher L. Morris, "Pion Physics at LAMPF," invited talk presented at the Third International Workshop on Pion-Nuclear Physics, Oberjoch, Federal Republic of Germany (August 1989).

M. G. Moshi, C. A. Whitten Jr., B. Aas, C. J. Igo, D. Lopiano, L. Ray, K. W. Jones, and M. M. Gazzaly, "Analy zing Powers for $P+{ }^{208} \mathrm{~Pb}$ at Large Momentum Transfers" (submitted to Physical Review C, October 1988).

D. Neuffer, E. Colton, G. Swain, H. Thiessen, B. Blind, R. Hardekopf, A. Jason, G. Lawrence, R. Shafer, T. Hardek, J. Hurd, R. Macek, and M. Plum, "Transverse Collective Instability in the PSR," Part. Accel. 23, 133 (1988).

D. Neuffer, "Nonlinear Fields, Particle Tracking, and Stability in the PSR," Los Alamos National Laboratory document AHF Technical Note 89-004 (August 1989). 
D. Neuffer, "Correction of the Multipole Content of Synchrotrons," Nucl. Instrum. and Methods A274, 400 (1989).

D. Neuffer and E. Forest, "A General Formalism of Quasi-L.ocal Correction of Multipole Distortions," Phys. Lett. A 135, 197 (1989).

D. Neuffer and T. S. Wang, "Electron-Proton Instability in the PSR and AHF" (submitted to the Proceedings of the Accelerator Design Workshop, Los Alamos, New Mexico, February 20-25, 1989).

D. Neuffer, "A Novel Method for Correcting the SSC Multipole Problem," in Proceedings of the 1988 Summer Study on High-Energy Physics in the 1990's, Snowmass, Colorado, June 27-july 15, 1988, S. Jensen, Ed. (World Scientific, Singapore, 1989), ISBN 9971-50-841-9, pp. 446-549.

D. S. Oakley, M. R. Braunstein, J. J. Kraushaar, R. A. Loveman, R. J. Peterson, D. J. Rilett, and R. L. Boudrie, "Isospin Asymmetries in Pion Scattering to Isoscalar Giant Quadrupole States in Ni lsotopes," Phys. Rev. C 40, 859 (1989).

S. Oneda, K. Terasaki, and M. D. Slaughter, "On Broken Flavor Symmetry in Particle Physics," Los Alamos National Laboratory document LA-UR-89-810 (submitted to Physical Review D, January 1989).

J. C. Peng, M. J. Leitch, J. Kapustinsky, C. S. Mishra, J. E. Simmons, J. D. Bowman, T. K. Li, L. C. Liu, Z. F. Wong, C. Smith, and R. R. Whitney, "Measurement of Coherent Eta Meson Production in the Reaction $\pi^{-}+{ }^{3} \mathrm{He} \rightarrow \eta+t$," Los Alamos National Laboratory document LA-UR-89-2387 (submitted to Physical Review Letters, July 1989).

M. A. Plum, A. A. Browman, D. Brown, D. M. Lee, and C. W. McCabe, "FailSafe Ion Chamber Errant Beam Detector Tailored for Personnel Protection," Los Alamos National Laboratory document LA-UR-89-1183 (submitted to the Proceedings of the 1989 IEEE Particle Accelerator Conference, Chicago, Illinois, March 1989).

M. A. Plum, R. A. Lindgren, J. Dubach, R. S. Hicks, R. L. Huffman, B. Parker, G. A. Peterson, J. Alster, J. Lichtenstadt, M. A. Moinester, and H. Baer, "180" Electron Scattering from ${ }^{14} \mathrm{C}$," Phys. Rev. 40, 1861 (1989).

M. A. Plum, A. A. Rrowman, D. Brown, D. M. Lee, and C. W. McCabe, "FailSafe Ion Chamber Errant Beam Detector Tailored for Personnel Protection," in Proceedings of the IEEE 1989 Particle Accelerator Conference, Chicago, Illinois (March 1989). 
R. L. Poirier and G. Swain, "Hardware Session and RF' Workshop Summary," in Proceedings of the Accelerator Design Workshop, Los Alamos, New Mexico, February 22-27, 1988, Los Alamos National Laboratory report LA-11432-C (January 1989).

A. M. Portis, D. W. Cooke, and H. Piel, "Microwave Surface Impedance of Granular Superconductors," Los Alamos National Laboratory document LA-UR-892697 (submitted to Physica C, August 1989).

S. Raby, G. B. West, and C. M. Hoffman, "Are There Really Any Experimental Limits On A Light Higgs Boson?," Phys. Rev. D 39, 828 (1989) and Nuovo Cimento 102A, 113 (1989).

J. Rapaport, P. W. Lisowski, J. L. Ullmann, R. C. Byrd, T. A. Carey, J. B. McClelland, L. J. Rybarcyk, T. N. Taddeucci, R. C. Haight, N. S. P. King, G. L. Morgan, D. A. Clark, D. E. Ciskowski, D. A. Lind, R. Smythe, C. D. Zafiratos, D. Prout, E. R. Sugarbaker, D. Marchlenski, W. P. Alford, and W. G. Love, "Fermi and Gamow-Teller Strength in $p$-Shell Nuclei from $(p, n)$ Reactions at 492 and $590 \mathrm{MeV}, "$ Phys. Rev. C 39, 1929 (1989).

H. F. W. Sadrozinski, W. A. Rowe, A. Seiden, E. Spencer, C. M. Hoffman, D. F.oltkamp, W. W. Kinnison, W. F. Sommer, Jr., and H. J. Ziock, "Test of Radiation Hardness of DMOS Transistors Under Neutron Irradiation," Los Alamos National Laboratory document LA-UR-89-295 (submitted to Proceedings of the Fifth European Symposium on Semiconductor Detectors, Munich, West Germany, February 21-23, 1989).

V. D. Sandberg, T. N. Thompson, and F. A. Helvy, "Report on the Performance and Operating Characteristics of the Burle C83061E Quantacon ${ }^{\text {TM }}$ Photomultiplier Tube," Los Alamos National Laboratory document LA-UR-89-2404 (submitted to the Proceedings of the Workshop on Physics and Experimental Techniques of High-Energy Neutrinos and VHE and UHE Gamma-Ray Particle Astrophysics, Little Rock, Arkansas, May 11-13, 1989, and to be published in Nuclear Physics B, 1989).

G. H. Sanders, "Precision Electroweak Physics with Neutrinos at Los Alamos," Los Alamos National Laboratory document LA-UR-89-1918 (submitted to the Proceedings of the XXIVth Recontres De Moriond, Electroweak Interactions and Unified Theories, Les Arcs, France, March 5-12, 1989).

G. H. Sanders, " $K_{L}^{0} \rightarrow \mu \mu$ Polarization: Future Prospects," Los Alamos National Laboratory document LA-UR-89-1892 (submitted to the Proceedings of the XXIVth Recontres de. Moriond, Electroweak Interactions and Unified Theories, Les Arcs, France, March 5-12, 1989). 
C.. Sanders, "Searching for Extensions to the Standard Model in Rare Kaon Decays," Los Alamos National Laboratory LA-UR-89-1256 (invited talk, in Proceedings of the Advanced Hadron Facility Design Workshop, Los Alamos, New Mexico, February 20-25, 1989).

L. Santi, M. Barlett, D. Ciskowski, R. Garfagnini, M. M. Gazzaly, G. W. Hoffmann, K. W. Jones, M. A. Nasser, G. Pauletta, C. Smith, N. Tanaka, and R. Whitney, "Evidence for Narrow Structure in the Analyzing Power of the ${ }^{3} \mathrm{He}(\vec{p}, d) \mathrm{X}$ Reaction," Phys. Rev. C 38, 2466 (1988).

M. Sataka, S. Ohtani, D. R. Swenson, and D. C. Gregory, "Experimental Cross Sections for Electron-Impact Ionization of Chromium Ions $\mathrm{Cr}^{6+}, \mathrm{Cr}^{7+}, \mathrm{Cr}^{3+}$, and

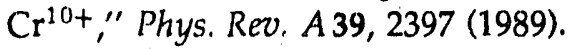

G. Schaffer, "Cost of a Normalconducting Linac Structure," Los Alamos National Laboratory document LA-UR-89-1901 (submitted to Proceedings of the Accelerator Design Workshop, Los Alamos, New Mexico, February 20-25, 1989).

G. Schaffer, "Update of Linac Cost Estimates," Los Alamos National Laboratory document LA-UR-89-1902 (June 1989).

G. Schaffer, "Cost Projection for a Superconducting Linac Structure," Los Alamos National Laboratory document LA-UR-89-1903 (submitted to the Proceedings of the Accelerator Design Workshop, Los Alamos, New Mexico, Febuuary 20-25, 1989).

G. Schaffer, "Cost of LAMIF Linac Options," Los Alamos National Laboratory document LA-UR-89-1904 (June 1989).

G. Schaffer, "Summary of Linac Group Discussions," Los Alamos National Laboratory document LA-UR-89-1956 (submitted to the Proceedings of the Accelerator Design Workshop, Los Alamos New Mexico, February 20-25, 1989).

S. C. Schaller, "Providing Common Data Access for the LAMPF and PSR Control System," Los Alamos National Laboratory document LA-UR-89-3643 (submitted to the Proceedings of the International Conference on Accelerator and Large Experimental Physics Control Systems, Vancouver, British Columbia, Canada, October 30November 3, 1989).

D. E. Schultz, "Creating the Next-Gen?ration Control System Software," Los Alamos National Laboratory document LA-UR-89-3630 (submitted to the Proceedings of the International Conference on Accelerator and Large Experimental Physics Control Systems, Vancouver, British Columbia, Canada, October 30-November 3, 1989). 
D. E. Schultz, "The Development of an Expert System to Tune a Beam Line," Los Alamos National Laboratory document LA-UR-89-3534 (submitted to the Proceedings International Conference on Acceleratur aril Large Experimental Physics Control Systems, Vancouver, British Columbia, Cinnada, October 30-November 3, 1989).

D. E. Schultz and P. A. Brown, "Using Expert System Techniques to Tune the ISIS Beam Line," Los Alamos National Laboratory document LA-UR-89-3533 (1989).

S. J. Seestrom, C. D. Bowman, R. Mortensen, J. D. Bowman, J. J. Szymanski, J. Knudson, S. Penttilä, V. W. Yuan, J. E. Bush, C. M. Frankle, S. R. Gould, D. G. Haase, C. E. Mitchell, N. R. Roberson, X. Zhu, P. P. J. Delheij, H. Postma, and B. Tippens, "Tests of Parity and Time Reversal Invariance in Neutron-Nucleus Scattering," Los Alamos National Laboratory document LA-UR-90-519 (submitted to the Workshop on Polarized Beams and Polarized Targets, McCormick's Creek, Indiana University Cyclotron Facility, Indiana, October 16-19, 1989).

R. E. Shafer, G. Swain, H. A. Thiessen, and R. Macek, "Beam Position Monitor Upgrade for the Los Alamos Proton Storage Ring," Los Alamos National Laboratory document LA-UR-89-1406 (submitted to the Proceedings of the 1989 Particle Accelerator Conference, Chicago, Illinois, March 20-23, 1989).

E. B. Shera, M. V. Hoehn, G. Fricke, and G. Mallot, "Nuclear Charge Radii of the Te Isotopes from Muonic Atoms," Phys. Rev. C 39, 195 (1989).

B. V. Shulgín, A. V. Kruzhalov, I. N. Ogorodnikov, L. V. Viktorov, V. A. Maslov, C. Alexander, and D. W. Cooke, "Scintillation Properties of B,2O Single Crystals," Zh. Prikl. Spektrosk. 49, 286 (1988).

R. L Shypit, D. V. Bugg, A. H. Sanjari, D. M. Lee, M. W. McNaughton, P. Riley, and C. A. Davis, "Spin Dependence of $p p \rightarrow n p \pi^{+}$from 492 to $796 \mathrm{MeV}, "$ Phys. Rev. C 40, 2203 (1989).

W. F. Sommer, F. A. Garner, R. D. Brown, and M. S. Wechsler, "Radiation Effects at a High-Power Accelerator and Applications to Advanced Energy Sources," Los Alamos National Laboratory document LA-UR-89-3615 (submitted to the 4th International Conference on Fusion Reactor Materials, Kyoto, Japan, December 3-7, 1989).

G. Swain, "Main Ring RF Cavity," in Proceedings of the 1988 Summer Study on High-Energy Physics in the 1990's, Snowmass, Colorado, June 27-July 15, 1988, S. Jensen, Ed. (World Scientific, Singapore, 1989), ISBN 9971-50-841-9, p. 458. 
G. Swain, "Design of a Main Ring Cavity," in Proceedings of the Accelerator Design Workshop, Los Alamos, New Mexico, February 22-27, 1988, Los Alamos National Laboratory report LA-11432-C (January 1989).

G. Swain, P. Busch, and M. Burns, "Generating Catalogs of Transverse Matching Solutions," Los Alamos National Laboratory document LA-UR-89-944 (submitted to the Proceedings of the 1989 Particle Accelerator Conference, Chicago, Illinois, March 20-23, 1989).

G. Swain, R. Kandarian, H. A. Thiessen, R. Poirier, and W. R. Smythe, 'Progress on a Prototype Main Ring RF Cavity," Los Alamos National Laboratc:y document LA-UR-89-976 (submitted to the Proceedings of the 1989 Particle Accelerator Conference, Chicago, Illinois, March 20-23, 1989).

G. R. Swain, "Improvements to a Transmission-Line Model of a Main-Ring Cavity," Los Alamos National Laboratory document LA-UR-89-1497 (submitted to the Proceedings of the Accelerator Design Workshop, Los Alamos New Mexico, February $20-25,1989)$.

G. Swain, "Time and Frequency Response of Beam Position Monitors at the Los Alamos Proton Storage Ring," Los Alamo: National Laboratory document LAUR-89-1574 (submitied to the Proceedings of the Accelerator Design Workshop, Los Alamos New Mexico, February 20-25, 1989).

G. Swain, "Usc of the Delta-t Method for Setting RF Phase and Amplitude for the AHF Linac," Los Alamos National Laboratory document LA-UR-89-1599 (submitted to the Proceedings of the Accelerator Design Workshop, Los Alamos, New Mexico, February 20-25, 1989).

G. Swain, "Tentative Design of Beam Focusing for the AHF Linac and Transport Systems," Los Alamos National Laboratory document L.A-UR-89-1600 (submitted to the Proceedings of the Accelerator Design Workshop, Los Alamos, New Mexico, February 20).25, 1989).

T. N. Taddeucci, "Charge Exchange Physics at LAMPF: Recent Results from NTOF," invited talk presented at the Gordon Conference, Tilton, New Hampshire, July 16,-.21, 1989.

Nobuyuki Tanaka, "Some Comments and Cost Estimate on the CEBAF HighResolution Spectrometer Facility," Los Alamos National Laboratory report LA11640-MS (October 1989). 
Nobuyuki Tanaka, "Spectrometers at LAMPF," talk presented at the Institute of High-Energy Physics, Serpukhov, Moscow USSR, September 13, 1989, Los Alamos National _aboratory document LA-UR-89-2688 (1989).

Nobuyuki Tanaka, "Polarized Proton end Antiproton Experiments at Energy $185 \mathrm{GeV}$ at Fermilab E-581/704," invited talk presented at the Institute of High Energy Physics, Moscow, USSR, September 4, 1989, and at the Institute of Nuclear Physics, Leningrad, USSR, September 13, 1989, Los Alamos National Laboratory document LA-UR-89-2884 (1989).

Nobuyuki Tanaka, "The Design and Performance of the FNAL High-Energy Polarized Beam Facility," talk presented at the 1989 IUCF "ropical Cunference, McCormick's Crcek State Park, Spencer, Indiana, October 16-18, 1989, Los Alamos National Laboratory document LA-UR-89-3600 (1989).

C. Y. .ang, P. Harris, H. C. Bryant, A. H. Mohagheghi, C. R. Quick, J. B. Donahue, R. A. Recder, Stanley Cohen, W. W. Smith, and J. E. Stewart, "Observation of Multiphoton Detachment of the $\mathrm{H}^{-}$Ion," Phys. Rev A 39, 6068 (1989).

H. A. Thiessen, "Trip Report on Snowmass APS Workshop: Physics in the 1990's," LOS Alamos National Laboratory document LA-UR-88-2619 (August 1988).

H. A. Thiessen, "Technical Summary of the Accelerator Design Workshop, 2227 February 1988," in Proceedings of the Accelerator Design Workshop, Los Alamos, New Mexico, Los Alamos National Laboratory report LA-11432-C (January 1989).

H. A. Thicssen, "Towards an Advanced Hadron Facility at Los Alamos," in Proceedings of the Accelerator Design Workshop, Los Alamos, New Mexico, Los Alamos National Laboratory report LA-11432-C (January 1989).

H. A. Thiessen, "Summary of Joint SSC/Kaon Factory Linac Injector Working Group," in Proceedings of the 1988 Summer Study on High-Energy Physics in the 1990's, Snowmass, Colorado, June 27-.July 15, 1988, S. Jensen, Ed. (World Scientific, Singapore, 1989)), ISBN-9971-50-841-9, pp. 441-442.

H. A. Thiessen, "Towards an Advanced Hadron Facility at Los Alamos," in Proceedings of the 1988 Summer Study on High-Energy Physics in the 1990's, Snowmass, rolorado, June 27-July 15, 1988, S. Jensen, Ed. (World Scientific, Singapore, 1989), ISBN-9971-5()-841-9, p. 408.

O. B. van Dyck, R. L. Hutson, M. E. Schillaci, and D. H. White, Eds., "Progress at LAMPF, January-December 1988," Los Alamos National Laboratory report LA-1 167)-IPR (September 1989). 
L. S. Walling, D. E. McMurry, D. V. Neuffer, and H. A. Thiessen, "Transmission Line Impedance Measurements for an Advanced Hadron Facility," Nucl. Instrum. and Methods A 281, 433 (1989).

L. S. Walling, D. McMurry, H. A. Thiessen, and D. Neuffer, "CouplingImpedance and Response-Characterization Measurements of a Proton Storage Ring Wall-Current Monitor," Los Alamos National Laboratory document LAUR-89-936 (submitted to the Proceedings of the 1989 Particle Accelerator Conference, Chicago, Illinois, March 20-23, 1989).

L. Walling, D. McMurry, and A. Shapiro, "PSR Current Monitor Measurements," Los Alamos National Laboratory document AHF Technical Note 89-003 (May 1989).

T.-S. Wang, E. Colton, and D. Neuffer, "The Tune Dependence of the Stability Threshold Current in the PSR," in Proceedings of the Accelerator Design Workshop, Los Alamios, New Mexico, February 22-27, 1988, Los Alamos National Laboratory report LA-11432-C (january 1989).

T.-S. Wang, E. Colton, A. Lombardi, D. V. Neuffer, and H. A. Thiessen, "Observation of the Tune Dependence of the Stability Threshold Current in the PSR," Los Alamos National Laboratory document LA-UR-89-981 (submitted to the Proceedings of the 1989 Particle Accelerator Conferenie, Chicago, Illinois, March 20-23, 1989).

Z. Weinfeld, E. Piasetzky, M. J. Leitch, H. W. Baer, C. S. Mishra, J. R. Comfort, J. Tinsley, and D. H. Wright, "Angular Distributions of Pion-Induced Transitions to Double-Isobaric-Analog-States on $1 f_{7 / 2}$ Shell Nuclei at $35 \mathrm{MeV}^{\prime \prime}$ (submitted to Physics Letters, July 1989).

Z. Weinfeld, E. Piasetzky, M. J. Leitch, H. W. Baer, C. S. Mishra, J. R. Comfort, J. Tinsley, and D. H. Wright, "Pion-Induced Transitions to Double-Isobaric-Analog States at $35 \mathrm{MeV}$ on $1 f_{7 / 2}$-Shell Nuclei," Los Alamos National Laboratory document LA-UR-89-3494 (submitted to Physics Letters B, October 1989).

D. H. White and D. Sullivan, "The Weak Interactions from 1950 to 1960: A Quantitative Bibliometric Study of the Formation of a Field," in Pions to Quarks, M. Brown, M. Dresden, and L. Hoddeson, Eds. (Cambridge University Press, New York, 1989), pp. 390-408.

D. H. White, "The Neutrino Program at LAMPF," Los Alamos National Laboratory document LA-UR-90-106 (submitted to the Proceedings of the International Seminar on Intermediate Energy Physics (INES-89), Moscow, USSR, November 2730, 1989). 
A. L. Williams, L. Agnew, L. G. Atencio, H. W. Baer, M. Burlein, G. R. Burleson, K. S. Dhuga, H. T. Fortune, G. S. Kyle, J. A. McGill, C. F. Moore, C. L. Morris, S. Mordechai, J. M. O'Donnell, M. W. Rawool, S. Schilling, J. D. Silk, and J. D. Zumbro, "Pion Double Charge Exchange Above $\Delta(1232)$ Resonance," Phys. Lett. 216, 11 (1989).

J. E. Wilmarth and L. J. Marek, "Fill Control System for Superconducting Solenoids at LAMPF Using a Programmable Controller," Los Alamos National Laboratory document LA-UR-89-3559 (submitted to the Proceedings of the International Conference on Accelerator and Large Experimental Physics Conirol Systems, Vancouver, British Columbia, Canada, October 30-November 3, 1989).

Sung Hoon Yoo, A. Williams, S. Mordechai, C. Fred Moore, C. L. Morris, S. J. Seestrom-Morris, M. Jones, S. Sterbenz, D. Dehnhard, D. S. Oakley, and A.

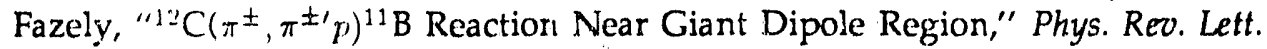
63, 738 (1989).

Sung Hoon Yoo, A. Williams, S. Mordechai, C. Fred Moore, C. L. Morris, S. J. Seestrum, M. Jones, S. Sterbenz, D. Dehnhard, D. S. Oakley, and A. Fazely, "208 $\mathrm{Pb}\left(\pi^{ \pm}, \pi^{ \pm \prime}\right)$ Reaction Near the Giant-Resonance Region," Los Alamos National Laboratory document LA-UR-89-3585 J. Phys. G 16, L89 (1990).

V. Yuan and J. D. Bowman, "Analytical Description of Scattering and Charge Stripping Processes in Thin Neutralizers," Los Alamos National Laboratory document LA-CP-89-210 (submitted to LA-CP Limited Distribution, December 1989).

H.-J. Ziock, C. M. Hoffman, D. Holtkamp, W. W. Kinnison, E. C. Milner, W. F. Sommer, J. DeWitt, A. Kaluniacki, H. Kolanoski, D. Pitzi, W. A. Rowe, H. F. W. Sadrozinski, E. Spencer, P. Tenenbaum, P. Ferguson, P. Giubellino, and S. Sartori, "Mcasurement of Proton-Induced Radiation Damage to CMOS Transistors and Pin Diodes" (submitted to the Proceedings of the IEEE 1989 Nuclear Science Symposium, San Francisco, California, January 15-19, 1990).

J. D. Zumbro, H. A. Thiessen, C. L. Morris, and J. A. McGill, "Uses of Super. conducting RF Structures in Pion Beams at LAMPF," Nucl. Instrum. and Methods B 40/41, 896 (1989). 


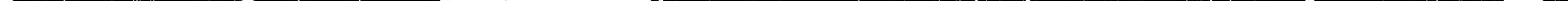




\section{OPPIS: The Optically Pumped Polarized Ion Source}

\section{R. L. York, D. R. Swenson,} D. Tupa, and O. van Dyck (Los Alamos)
OPPIS, the new polarized-beam ion source, was commissioned in 1989 . We will describe the source configuration and performance as it was and as it is planned for 1990. In addition, the P- beam chopper system, also commissioned this year, will be mentioned. This report is abstracted from several recent detailed papers. ${ }^{1-4}$

OPPIS development was started in 1986. By the end of that year, the basic source configuration was determined and is described in that year's Progress Report. ${ }^{5}$ Among the essential features are use of a bending magnet at the source exit, as shown in Fig. 1, so that the laser beam can be inserted on the source ax $s$ without aperture losses on the ECR extraction electrodes, and high vacuum pumping speed to reduce background beam. To obtain a high magnetic ficld with adequate aperture, a superconducting magnet was chosen for the alkalivapor polarizer region.

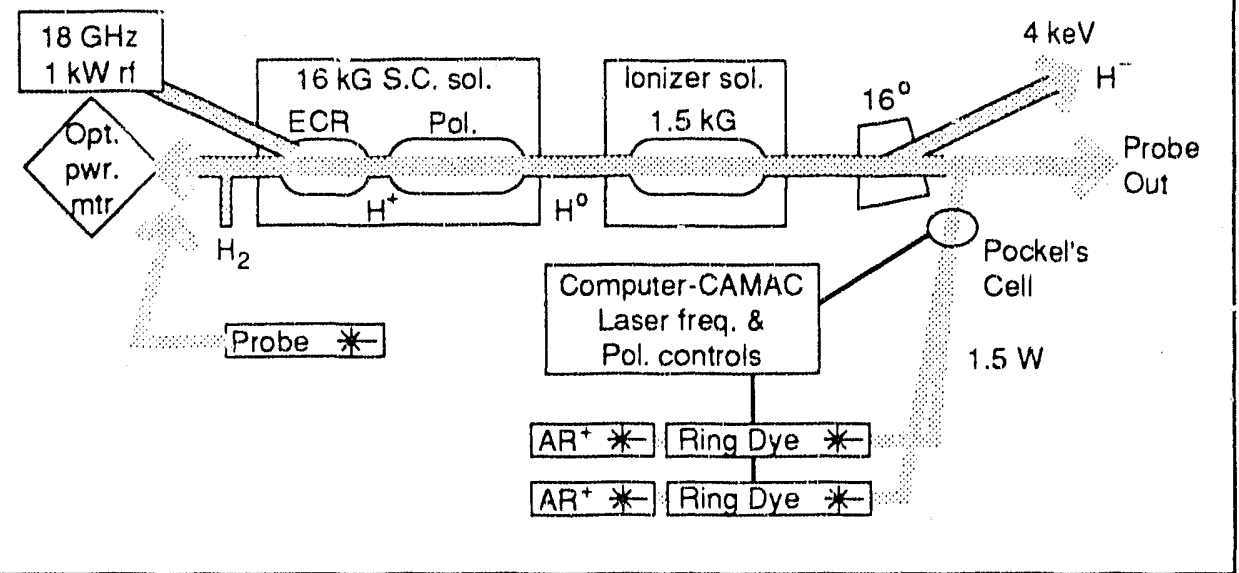

Fig. 1. OPPIS configuration for 1989 operation. The laser beam is absorbed by the sodium vapor in the polarizer cell. The sodium vapor transfers polarized electrons to protons from the ECR source to make a beam of polarized atoms. In the ionizer region, the beam picks up another electron to become $\mathrm{H}^{-}$. Aulomatic spin reversal is provided by computer control of laser frequency and laser beam polarization.

In 1987-88, a laser system was assembled and basic optical pumping measurements were made on sodium vapor. At the end of 1988 LAMPF operation, the Lamb-shift source was removed from the P- dome and OPPIS was installed. OPPIS beam was run intermittently in the linac during summer 1989 operation to assess performance using the Line B polarimeters. By September, we had achieved adequate intensity and polarization with sufficiently stable operation to undertake a research demonstration run. During September 20-29, OPPIS beam ran steadily at $4 \mu \mathrm{A}$ peak (at $750 \mathrm{keV}$ ), $45 \%$ polarization on target at $800 \mathrm{MeV}$, 
and $85 \%$ availability. This beam was used to complete a phase of Exp. 1040, a measurement of ${ }^{14} \mathrm{C}$ analyzing power.

During this time, the new $\mathrm{P}-$ chopper system was commissioned and provided 200-ns pulse spacing for Exp. 1040 on the NTOF line. The cliopper system uses the 10-MHz two-stage buncher available since 1987 to compress the injected beam into 100-ns micropulses. It can select micropulses at any multiple of that period.

The 1989 OPPIS configuration used $\mathrm{Na}$ as the polarization exchange medium. As sketched in Fig. 1, laser light is absorbed by one of the two Zeeman levels of the $\mathrm{Na}$ vapor at $16 \mathrm{kG}$ in the polarizer cell. The circular polarization orientation (photon helicity) of the laser light is set by the Pockel's cell, which is a voltage-controlled variable retarder element. Ion beam spin reversal is accomplished by changing the laser frequency and polarization orientation for absorption on the other Zeeman level. The vapor polarization could be monitored with the optical power meter, which gives the amount of laser light absorbed by the vapur, or alternatively, could be directly measured by optical rotation of the probe beam.

Tunable single-frequency dye lasers were used to optically pump the $\mathrm{Na}$ vapor. These complicated and expensive instruments produce approximately $1 \mathrm{~W}$ per laser at absorption frequency of $\mathrm{Na}$, which has proven insufficient to polarize $\mathrm{Na}$ targets of adequate thickness. For OPPIS, the $\mathrm{P}^{2} \mathrm{I}$ performance (a common figure of merit of polarized ion sources) is directly related to laser power. With $1.5 \mathrm{~W}$ of power typically available from two laser beams combined in the ion source, we believe the performance achieved in 1989 is near the maximum attainable. The results achieved represent a factor of two improvement in $\mathrm{P}^{2} \mathrm{I}$ over the Lamb-shift source but does not meet expectations for 1990.

Thus, work at the beginning of the LAMPF shutdown focused on changeover of the optical pumping system to more powerful solid-state lasers that had just become commercially available. The closest spectral match to a suitable alkali is obtained from titanium-doped sapphire lasers, which cover the near-IR and deliver over $3 \mathrm{~W} \mathrm{cw}$. By the end of the year, the source had been changed over to optically pump potassium and the initial results encouraged us to commit to this configuration for 1990.

Given a vapor thickness in the polarizer cell limited by available laser power, the final beam current can also be increased by more efficient ion beam optics. In OPPIS (as in the Lamb-shift source) a large loss of particle-beam flux occurs between polarizer (neutralizer) and ionizer. The key to minimizing this loss is in optimizing the $\mathrm{H}^{+}$extraction optics so that the neutral particle trajectories are not too divergent. A lengthy process of iterating on electrode design and biasing arrangements has yielded significant gain in beam current. Additional gains are possible through further optimization of the extraction optics, source alignment, and downstream optics. 
These recent developments, in combination, should allow the source to meet or substantially exceed the performance goal for 1990 operation of $60 \%$ polarization and $10-\mu \mathrm{A}$ peak current. Similar developments in future years should yield additional substantial factors of improvement to give LAMPF an outstanding polarized beam capability.

\section{References}

1. D. R. Swenson, D. Tupa, O. van Dyck, T. J. Rosson, and R. L. York, "Recent Developments in Optical Pumping in OPPIS at LAMPF," Los Alamos National Laboratory document LA-UR-90-396 (to be published in Proceedings of the International Workshop on Polarized Ion Sources and Polarized Gas Jets, KEK, Tsukuba, Japan, February 12-17, 1990).

2. R. L. York., "Rncent Developments in Optically Pumped Polarized Ion Sources," Los Alımos National Laboratory document LA-UR-90-397 (to be published in Proceedings of the International Workshop on Polarized Ion Sources and Polarized Gas Jets, KEK, Tsukuba, Japan, February 12-17, 1990).

3. R. L. York, O, van Dyck, D. R. Swenson, and D. Tufa, "The LAMPF Optically Pumped Polarized Ion Source," Los Alamos National Laboratory document LA-UR-90-398 (to be published in Proceedings of the International Workshop on Polarized Ion Sources and Polarized Gas Jets, KEK, Tsukuba, Japan, February 12 17, 1990).

4. O. van Dyck, "LAMPF Polarized Beams and Facilities," Los Alamos National Laboratory document LA-UR-89-3844 (to be published in Proceedings of INES89, Moscow, USSR, 1989).

5. "Progress at LAMPF, January-December 1986," Los Alamos National Laboratory report LA-11048-PR (1987), p. 256. 
LAMPF and the Proton Storage Ring (PSR) Control Systems and Operations

\author{
M. Hoehn (Los Alamos)
}

When MP-6 was formed in January 1988, there was an effort to combine functions that were performed at LAMPF with those same functions performed in MP-5 for the Proton Storage Ring (PSR), with the goal of providing iniproved efficiency in operations and merging the talents and capabilities of the people in the controls software support effort. The results of the reorganization in achieving the goals has had great success. Operators rotate through shifts at each of the three control rooms (Central Control Room, Injector Control Room, and LANSCE Control Room). As a result, they have improved beam availability by increasing their understanding of the interactions between beam delivery systems at LAMPF and PSR.

The LAMPF and the PSR control systems are significantly different in many respects, and this means that operators must know two different control systems. Some of the more significant differences include the following:

- The LAMPF system was designed in the early 1970's and the PSR system was designed in the early 1980's by different sets of people. This resulted in different hardware being employed in the two systems, as ivell as different software philosophies.

- The LAMPF system obtains data from devices "on demand," whereas the PSR system maintains a "live" data base of the most recent data.

- The LAMPF system uses RICE and an ethernet network to distribute computers at various places in the facility, whereas the PSR system uses CAMAC serial highways.

The MP-6 Controls Section has been attempting to make the two systems more compatible. During the past year, since the PSR and LAMPF control systems staff were combined, we have made some progress in integrating the two control systems:

- Most PSR devices can be read and controlled from the LAMPF consoles. This immediately allowed operators in the LAMPF Central Control Koom to monitor effects of charges in the beam in the linac on the beam delivered to PSR. This also provided ability to use the LAMPF system device monitoring program, DATASCAN, to analyze a long-standing problem with magnet power supply drifts and to correlate radiation levels with beam current levels in PSR.

- Two LAMPF system consoles have been installed on the PSR control computer so that the beam in the linac can be observed directly in the PSR control room.

- The PSR wire scanners are now run from one of the LAMPF consoles on the PSR system using a VAXELN CAMAC system and a LAMPF program modified for PSR. This has significantly speeded up acquisition of wire-scanner data at PSR, àn important improvement since the previous method often failed when the beam tripped off during a scan. 
FACILITY DEVELOPMENT

LAMPF Proton Storage Ring (PSR)

A goal for the future is to create a common software interiace to both systems and then to develop a common operator interface, perhaps using workstations. 


\section{PSR and WNR Beam Delivery Systems}

\author{
R. J. Macek (Los Alamos)
}

\section{PSR (LANSCE Beam Delivery System)}

Substantial improvements in the performance of the PSR were achieved in FY 1989. Operating currents were increased from $35 \mu \mathrm{A}$ to $64 \mu \mathrm{A}$ and availability during the entire 1989 running cycle averaged $74 \%$. The international external review committee for the PSR in a recent (July 1989) mini-review noted with satisfaction the improved status of the PSR. The increased Institutionally Supported Rescarch and Development (ISRD) funds for PSR development were a major contribution to the improved rate of progress at the PSR in FY 1989.

\section{Table I. LANSCE Production Statistics for 1989.}

Scheduled Beam Hours $\quad 2,136$

Available Beam Hours

$1,581.2$

Downtime of LANSCE Target System and Interlocks (hr)

21.6

Delivered Beam Hours

Delivered $\mu \mathrm{A}$ Hours

78,121

Average Beam Availability

$74 \%$

Average seam Current on LANSCE Target $(\mu \mathrm{A})$

50.1

Highest priority in 1989 was given to improving beam availability. The increase from an average of 50\% in FY 1988 to $74 \%$ in 1989 was brought about by a systematic and thorough assessment and cornection of numerous problems with a number of systems, including several pulsed power systems, the $\mathrm{H}^{-}$source and injector, DC magnets and power supplies, deionized water systems, air conditioning, and the computer controls system. Improved errant beam diagnostics, including the addition of an ion chamber-based fast protect system, were instrumental in reducing the number of trips of the Personal Safety System (PSS) and thereby increased operational reliability and efficiency. Improved regulation of bending magnet power supplies was crucial to reducing the numerous excursions of the beam stecring experienced in FY 1988 and also contributed to improved beam availability by reducing the amount of retuning needed to keep the beam injected properly into the PSR. Further improvement in magr et regulation is needed and is being implemented through the purchase of suitable power supplies for critical bending magnets.

Significant improvements to $\mathrm{H}^{0}$ injecticn have resulted in reduced beam loss rates and thus have increased beam intensity. One improvement was the development and successful implementation of offset injection (in the vertical dimension) and another was the development of fiber-supported, minimal-area stripper foils, the so-called "postage stamp" foil. Both developments reduced the probability for traversal of the stripper by the stored beam. Preliminary tests 
of a double-injection offset were also encouraging for further reduction of beam losses in the future when the stripper foil mechanisms can be suitably modified.

Beam losses during the first turn after injection now account for about $40 \%$ of the slow losses. The origin of the first-turn losses is still not understood, although numerous possibilities have been investigated and data are still being analyzed. Further progress in raising the beam intensity will be increasingly limited by the first-turn losses. It is now apparent that first-turn losses must be understood in detail and the means found to reduce them.

A successful model has been developed for describing the slow beam losses arising from traversal of the stripper foil by the stored beam. From this knowledge, methods for reducing foil traversals, such as offset injection and fibersupported foils, are being developed and are proving to be effective in reducing beam losses. However, these methods have little influence on the first-turn losses which may prove to be the ultimate limiting factor for $\mathrm{H}^{0}$ injection.

One of the goals of research in FY 1989 was to uncover the limits of the present $\mathrm{H}^{0}$ injection method. Based on present knowledge and practice, the limit for the PSR appears to be around $75 \mu \mathrm{A}$ at $20 \mathrm{~Hz}$. Elimination of the first-turn losses could raise the limit to $100 \mu \mathrm{A}$-hence the importance of understanding these losses. Furthermore, without knowledge of the origins of the first-turn losses, one cannot conclude with certainty that another attractive alternative, direct $\mathrm{H}^{-}$injection, would eliminate them. Further study of first-turn lo'sses is high on the list of priorities for FY 1990.

Our strategy for increasing beam intensity has emphasized reduction of beam losses, since it has been the resulting activation of ring components and the need for hands-on maintenance that set the operating intensity. Another approach, which we are also pursuing, is to develop capabilities for handling higher leveis of activation. The limit set by activation is not a sharp threshold; it is actually set by the amount of dose accumulated by personnel who must work with the hardware. Much can be done, short of full remote handling, to improve the situation. Higher reliability reduces the frequency of repair and thus dose to maintenance personnel. Components can be designed for rapid repair and replacement and can also be designed of materials that result in lower activation for a given level af beam loss. Some control of the location of the losses should be possible. Implementation of such a strategy is not quick and easy; considerable engineering research and prototype development is called for. Improvements are incremental but numerous possible improvements are continually being identified.

Development of a full-aperture extraction system based on ferrite kicker technology held the promise of increasing the horizontal acceptance of the PSR by a factor of two and thereby reduced beam losses s'gnificantly. Development of a prototype pulser for such a system received considerable effort in FY 1989. One result from this work is more rertain knowledge of the costs of this option, which are now known to be considerably higher than originally estimated. A fullaperture ferrite kicker system appears technically feasible but will cost between 
4-5\$M to achieve and is therefore not likely to be chosen as the next step in upgrading the PSR for $100 \mu \mathrm{A}$.

Further studies of beam instabilities at the PSR were carried out in FY 1989. The research was aided by the successful development of wide-band beam position monitors and implementation of a fast digitizing and data-acquisition system for transient phenomena. A large body of evide ce has been accumulated, which supports the hypothesis that the instability is caused by electrons interacting with the proton beam. A definitive test of this hypothesis is being sought, but one that is not as expensive as the cure, which would require the installation of a large number of clearing electrodes to remove the electrons as they are produced.

\section{Future Plans for the PSR}

In the context of the total program at LANSCE/PSR Ifunded by monies from Basic Encrgy Sciences (BES), Weapons, and ISRD sources], our highest priority for FY 1990 is to consolidate the gains in reliability and beam intensity achieved in FY 1989. Enhanced radiation safety is of paramount importance and will be improved by addition of more shielding over ER-1 (funded primarily by Weapons sources) and by further improvements to our state-of-the-art errant beam-detection instrumentation. Development of low-activation, highly reliable components for the PSR injection region will reduce dose to personnel who must work in this area.

Further exploration of the limits of $\mathrm{H}^{(1)}$ injection will be undertaken through development and exploitation of a double-injection of fset and study of the causes of the large "first-turn" losses. Research into the beam instabilities at the PSR will continue with a focus on definitive tests of the $c-p$ hypothesis. Development of advanced beam-diagnostics instrumentation needed for both operation and beam dynamics studies will continue.

Research and Development (R\&D) and project planning needed for an upgrade of the PSR to $100 \mu \mathrm{A}$ will be slowed because of reduced funding in FY 1990; however, we still expect to make some progress in this area. Implementation of the upgrade will be paced by funding. The ultimate goai of $5.2 \times 10^{13} \mathrm{ppp}$ will require further reduction of slow losses, contiol of beam instabilities and, in all likelihood, a higher intensity $\mathrm{H}^{-}$ion source. Additional $\mathrm{R} \& \mathrm{D}$ will be required to reach the ultimate goal which is not expected to be achieved until the mid-1990's.

\section{WNR Beam Delivery System}

Many of the same system improvements that greatly enhanced LANSCE beam availability also contributed to the gratifying improvements in WNR beam availability. An equally significant development for WNR was the upgrade of the switchyard kicker from $30 \mathrm{~Hz}$ to $60 \mathrm{~Hz}$. This development increased the macropulse repetition rate for WNR to $40 \mathrm{~Hz}$, a factor of 2.7 improvement. Production statistics for WNR are shown in Table II below. 
Table II. WNR Production Statistics for 1989.

Scheduled Beam Hours

2236

Available Beam Hours

1751

Average Beam Availability

$78 \%$

Additional Beam Hours Available During PSR Development

445

The Personal Safety System (PSS) for WNR was upgraded in two ways:

1. Improved errant beam instrumentation was added in the beam tunnel; and

2. An experimental area PSS was implemented that incorporated PSS interlocks on the access gates to experimental caves and detector sheds.

Area definition was also refined by additional fencing. These improvements greatly reduced the dependence on administrative controls for personnel protection. 


\section{1-MHz RF System Problems}

D. Doss and G. Tubb (Los Alamos)
LAMPF's 201-MHz Alvarez drift-tube linac (0.75-100 MeV) is powered by four gridded-tube of amplifier systems (See Fig. 1). The first module provides $375 \mathrm{~kW}$ peak power cluring tank fill time and about $275 \mathrm{~kW}$ during acceleration, while modules 2-4 must deliver $3 \mathrm{MW}$ peak power during tank fill time and about 2.6 MW during acceleration.

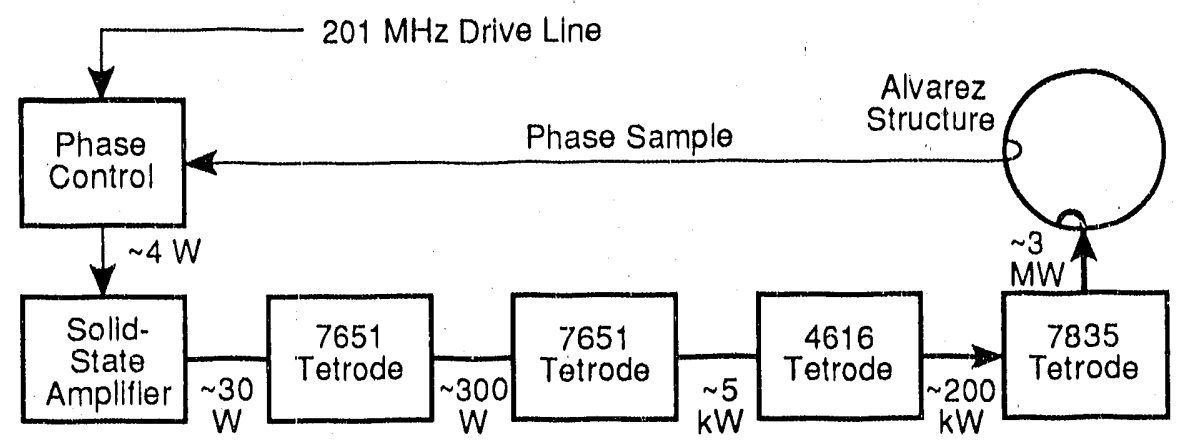

Fig. 1. Peak power levels are shown in this block-diagram of the existing 201-MHz amplifier system.

Maximum duty factor capability for these systems must be $12.8 \%$ for an accelerator beam duty factor of $10.4 \%$, the maximum beam duty factor ever used at LAMPF for any appreciable period of time. For a typical 7835-anode efficiency of $\sim 50 \%$ and the required peak output power of $3 \mathrm{MW}$ (into a matched load), peak anode dissipation is also $3 \mathrm{MW}$. An rf duty factor of $12.8 \%$ would result in an average dissipation of $384 \mathrm{~kW}$. Accelerator service is somewhat less stringent, since $3 \mathrm{MW}$ is provided only during tank fill time $(200 \mu \mathrm{s})$ and $2.6 \mathrm{MW}$ peak power is required during the interval when the beam is present $(870 \mu \mathrm{s})$. This would result in an average dissipation of $290 \mathrm{~kW}$, which is within the 300$\mathrm{kW}$ anode dissipation specifications for the 7835. Direct measurement of anode dissipation has also been made at various duty factors and these measurements verify that the tube is being operated under the $300-\mathrm{kW}$ dissipation level.

The four 201-MHz gridded-tube amplifier modules have been responsible for considerably more accelerator downtime than the $44805-\mathrm{MHz}, 1.25-\mathrm{MW}$ klystron moduies. The 201 systems also are responsible for a large proportion of the total downtime of the accelerator: $\sim 27 \%$ in 1988 and $\sim 13 \%$ in 1989 when duty factor was decreased to preserve the $7835 \mathrm{~s}$.

7835 tube problems have increased considerably since about 1984 (see Fig. 2). Because duty factor was increased yearly from 1977 until 1984 and then was leveled off between 1984 and 1989, it was first thought that the longer duty factor was the major contributing factor to tube loss. However, a closer look indicates that additional factors (such as 7835 quality) are also important. 


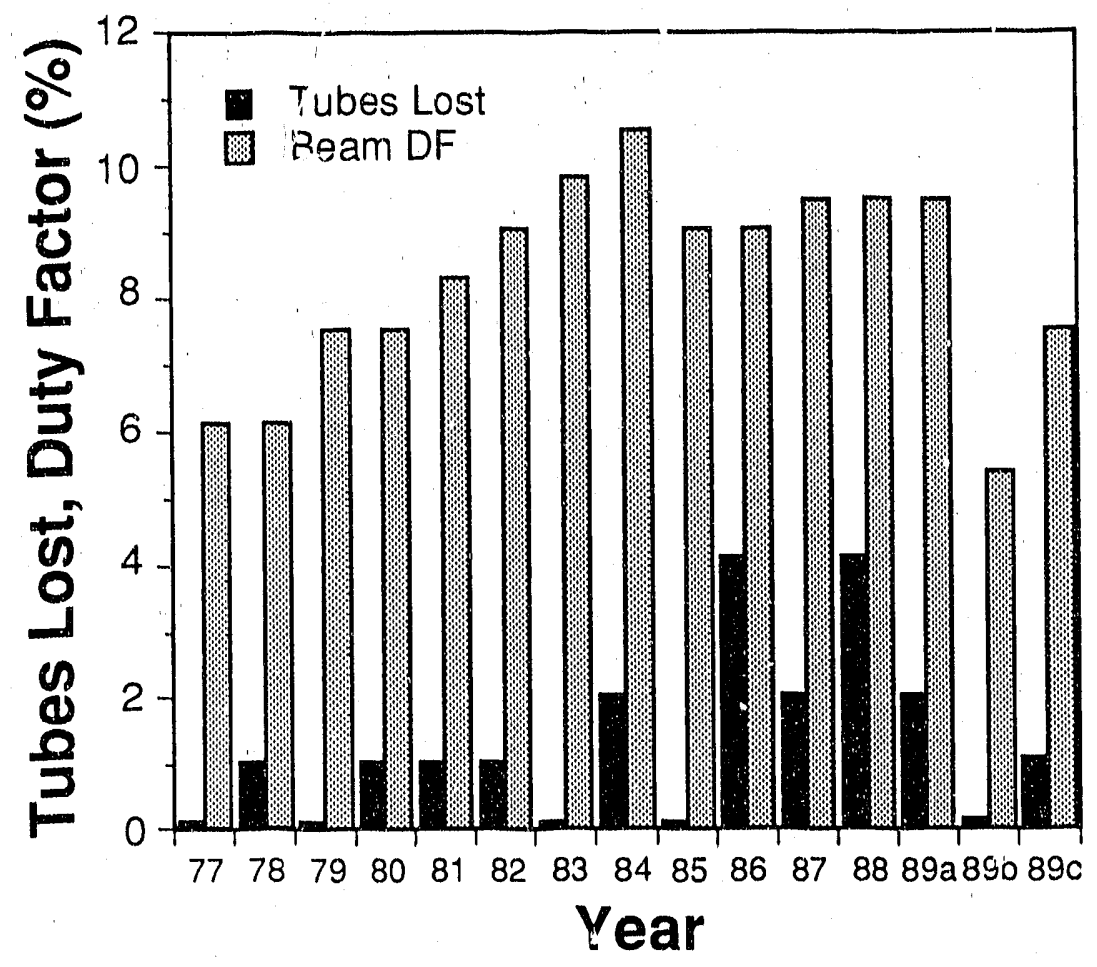

Fig. 2. 7835 failu s and beam duty factor by "'aar. (1989 is presented in three segments, refle: ting modifications in duty factor.)

Inspection of the date of manufacture of tubes that failed prior to 1984 and those that failed after 1984 rev als interesting results. Most of the tubes that failed after 1984 werc manufactur d after 1980. The Mean-Time-Between Failure (MTBF) for tubes manufactured prior to 1980 was $15,700 \mathrm{~h}$ while the MTBF for tubes manufactured after 1980 was $1460 \mathrm{~h}$. These data do not completely eliminate the question of duty factor since, for the period after 1980, both Fermi and Brookhaven have MTBFs of $\sim 10,000 \mathrm{~h}$. Both of those laboratories are operating their 7835 triodes at much lower duty factors than LAMPF. The Laboratory is working closely with the tube manufacturer to try to understand the decrease in 7835 life and to correct the problems.

In the interest of a long-term solution, we are evaluating the possibility of replacing the 7835 triodes with klystrons. This would require an industrial contract to develop 201-MHz klystrons and would be an expensive project for LAMPF. However, this approach also could help the other laboratories that depend upon these tubes. 
Computing Directions and Options for Data Acquisition and Analysis at LAMPF

\author{
M. Oothoudt and M. Hoenn \\ (Los Alamos)
}

In January 1989, a workshop was help for three days to discuss computing issues relevant to data acquisition and analysis at LAMPF. The committee, designed to represent a spectrum of computing interests and experiences, consisted of 14 members from the LAMPF community, plus representatives from Oak Ridge, Fermilab, Argonne, and Lawrence Berkeley Laboratory.

The Committee heard 27 reports on computing issues and discussed their relevance to the LAMPF experimental program. A report on the workshop has been issued ${ }^{1}$ summarizing the discussions and recommending computing directions appropriate for LAMPF.

Major recommendations include:

Data Acquisition:

- Methods to extend the capabilities of front-end electronics should be investigated and, where appropriate, implemented.

- CAMAC and the $Q$ data acquisition system should be the supported configuration for the vast majority of experiments expected during the next five years.

- Large experiments which cannot be supported by CAMAC/Q should, where appropriate, make use of facilities developed and supported for other large experiments at LAMPF.

Data Analysis:

- The goal of the DAC should be to provide sufficient computation time, disk space, and other resources to allow timcly processing of physics data, as well as support for administrative and daily computing activities.

- As an aid for planning, the official proposal form for experiments at LAMPF should be modified to require estimates of LAMPF computer resources needed for analysis of data.

- Current levels of consulting and trouble-shooting were found to be adequate.

- Supercomputer resources from C Division or the Office of Energy Research should be used, where appropriate and affordable, for problems too large to be efficiently analyzed at the DAC.

Networks:

- The current level of support and capabilities for the LAMPF local area network should be maintained.

- LAMPF should maintain good connections to external wide-area networks. 
Graphics, Workstations, and Human Interfaces:

- Better user interfaces and graphics capabilities should be investigated for implementation on workstations and/or color terminals.

- The DAC should devise a written plan for workstation support to avoid the need for a large increase in support staff.

- MP Division should not provide support for personal computers, since such support is available elsewhere in the Laboratory.

\section{Reference}

1. M. A. Oothoudt, M. V. Hoehn, J. F. Amann, C. B. Bemis, T. A. Carey, P. S. Cooper et al., 'Report from the Workshop on Computing Directions and Options for Data Analysis and Acquisition at LAMPF, Los Alamos National Laboratory report LA-11662-MS (October 1989). 


\section{Cost of the LAMPF Linac Extension}

\author{
G. Schaffer (Los Alamos)
}

During the design work on various layout options for a $60-\mathrm{GeV}$ Advanced Hadron Facility (AHF), several possibilities to increase the energy of the present $800-\mathrm{MeV}$ linac to $1.6 \mathrm{GeV}$ were investigated. In particular, these were:

1. An $800-\mathrm{MeV}$ linac extension (add-on linac), with normalconducting or superconducting accelerating structure; and, alternatively,

2. The replacement of part of the (normalconducting) side-coupled structure in the existing LAMPF tunnel by a superconducting structure wit? the highest possible gradient, to reach $1600-\mathrm{MeV}$ final energy.

The relative cost minima and absolute cost figures for these different options were developed and presented to the LAMPF Long Range Plan Accelerator and Facilities Committee in April 1989.

A normalconducting extension (using either $805 \mathrm{MHz}$ or $1207.5 \mathrm{MHz}$ operating frequency) would require $\$ 109 \mathrm{M}$ construction funds; a superconducting version (operating at $402.5 \mathrm{MHz}$ ) would cost $\$ 103 \mathrm{M}$; and a transformation in the existing LAMPF building $\$ 138 \mathrm{M}$ (in 1989 prices).

Following that presentation, we reviewed those preliminary cost estimates with experts in normalconducting structures from AT-1, FNAL, SAIC, and with personnel from CERN responsible for their tender for 20 superconducting cavities for the Low-Energy Pion (LEI) Channel. Our goal was a more precise cost estimate for an upgrade based on superconducting structures.

Details of our cost estimates were published in a number of reports (LA-UR$89-1901,-1902,-1903,-1904$, and -3713 ). The total construction cost for a superconducting $800-\mathrm{MeV}$ extension amounts to $\$ 99.5 \mathrm{M}$-buildings, cryoplant, and linac included. The corresponding figure for a technically comparable normalconducting structure (1207.5 MHz, with an on-axis-coupled structure) is $\$ 104.6 \mathrm{M}$.

While the superconducting version is based on a gradient of $5 \mathrm{MeV} / \mathrm{m}$ inside the active structure, the normalconducting version would operate at $3 \mathrm{MeV} / \mathrm{m}$ (cost minimum for construction). In both cases, the length of the linac building would be $300 \mathrm{~m}$.

The savings in electrical power consumption would be $\$ 2 \mathrm{M}$ per year for the superconducting version. It was found advisable to study in more detail the behavior of superconducting structures when they accelerate pulsed beams; the suppression of transients should be proven : practical tests by one or two sections in the LAMPF linac before a final decision is made.

It should be mentioned that there are also other promising possibilities to arrive at $1.6-\mathrm{GeV}$ beam energy with different scenarios in which the existing experimental areas would be used and no interruption of the operation of the present linac would be required. These options merit further investigation. 


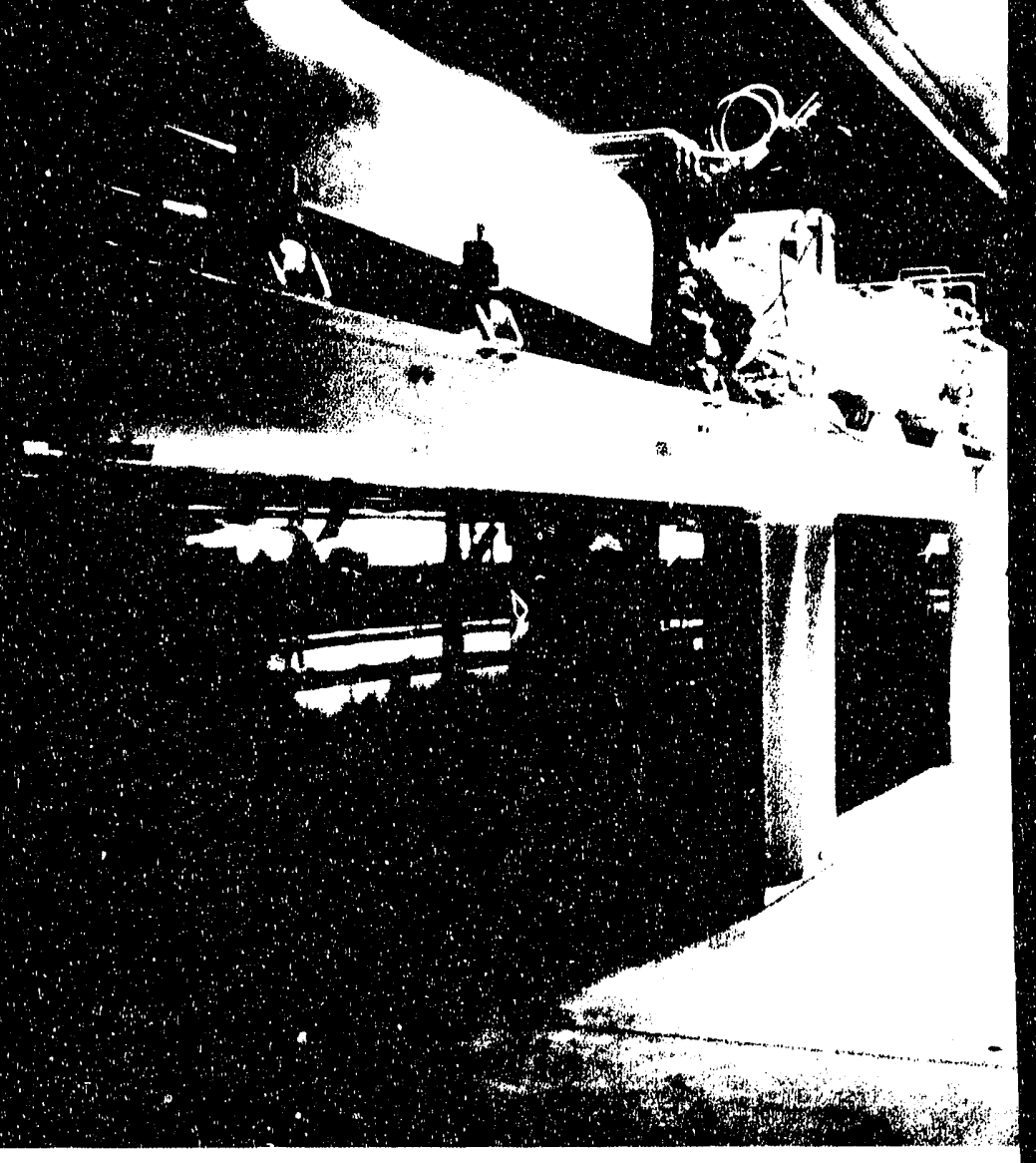




\section{Accelerator Operations}

David Helfer (Los Alamos)
This report covers operating cycles 53,54, and 55. The accelerator was in operation from May 4 through October 1, 1989. Beams were provided for research use for 156 days, and for facility development for four day:. A summary of information on beams provided for research is given in Table $\mathrm{I}$.

Table I. Beam Statistics for Cycles 53, 54, and 55.

\begin{tabular}{|c|c|c|c|}
\hline & Cycle 53 & Cycle 54 & Cycle 55 \\
\hline No. of experiments served ${ }^{a}$ & 16 & 16 & 21 \\
\hline $\begin{array}{l}\mathrm{H}^{+} \text {scheduled beam hours } \\
\mathrm{H}^{-} \text {PSR scheduled beam hours } \\
\mathrm{H}^{-} \text {Line } \mathrm{X} \text { scheduled beam hours } \\
\mathrm{P}^{-} \text {Line } \mathrm{X} \text { scheduled beam hours }\end{array}$ & $\begin{array}{r}1058 \\
624 \\
696 \\
-\end{array}$ & $\begin{array}{r}1152 \\
896 \\
656 \\
-\end{array}$ & $\begin{array}{r}1030 \\
814 \\
694 \\
120\end{array}$ \\
\hline $\begin{array}{l}\mathrm{H}^{+} \text {beam availability }(\%) \\
\mathrm{H}^{-} \text {PSR beam availability }(\%) \\
\mathrm{H}^{-} \text {Line } \mathrm{X} \text { beam availability }(\%) \\
\mathrm{P}^{-} \text {Line } \mathrm{X} \text { beam availability }(\%)\end{array}$ & $\begin{array}{l}87 \\
87 \\
81 \\
-\end{array}$ & $\begin{array}{l}77 \\
84 \\
84 \\
-\end{array}$ & $\begin{array}{l}91 \\
83 \\
77 \\
51\end{array}$ \\
\hline $\begin{array}{l}\mathrm{H}^{+} \text {average current }(\mu \mathrm{A}) \\
\mathrm{H}^{-} \text {PSR average current }(\mu \mathrm{A}) \\
\mathrm{H}^{-} \text {Line } \mathrm{X} \text { average current }(\mu \mathrm{A}) \\
\mathrm{P}-\text { Line } \mathrm{X} \text { average current }(\mathrm{nA})\end{array}$ & $\begin{array}{r}550 \\
45 \\
<1 \\
-\end{array}$ & $\begin{array}{r}900 \\
55 \\
<1 \\
-\end{array}$ & $\begin{array}{r}900 \\
60 \\
<1 \\
25\end{array}$ \\
\hline $\begin{array}{l}\mathrm{H}^{+} \text {beam duty factor }(\%) \\
\mathrm{H}^{-} \text {PSR beam duty factor }(\%) \\
\mathrm{H}^{-} \text {Line } \mathrm{X} \text { beam duty factor }(\%) \\
\mathrm{P}-\text { Line } \mathrm{X} \text { beam duty factor }(\mathrm{)}\end{array}$ & $\begin{array}{l}3.4 \\
0.8 \\
2.0 \\
-\end{array}$ & $\begin{array}{l}5.1 \\
0.8 \\
2.0 \\
-\end{array}$ & $\begin{array}{l}5.1 \\
0.8 \\
2.0 \\
2.0\end{array}$ \\
\hline
\end{tabular}

a Does not include experiments performed at the PSR-LANSCE/WNR areas.

A significant (50\%) reduction in downtime from the 201- $\mathrm{MHz}$ amplifier system was accomplished this year by reducing the $\mathrm{rf}$ duty and $\mathrm{H}^{+}$peak current. This duty and current reduction has resulted in a reduction of the average current figures as compared to those of previous years.

The Optically Pumped Polarized Ion Source (OPPIS) was commissioned during the run period. Twenty-five $n A$ of polarized beam wilh $45 \%$ polarization was delivered to the NPL areas at the end of cycle 55.

During CY 1989, protons and neutrons were delivered to the new Medium Resolution Spectrometer (MRS) facility for the first time. No major problems were encountered.

A surnmary of unscheduled facility downtime during research shifts is given in Table II. Because some of the outages were concurrent, and because some affected only one of the three beams, the total is much greater than the beam downtime. 
Table II. Unscheduled Machine Downtime.

\begin{tabular}{lcc}
\hline Category & $\begin{array}{c}\text { Downtime } \\
(\boldsymbol{h})\end{array}$ & $\begin{array}{c}\text { Percent } \\
\text { of Total }\end{array}$ \\
\hline 201-MHz amplifiers and transmission lines & 100 & 13 \\
805-MHz amplifier systems & 35 & 4 \\
Vacuum systems & 205 & 26 \\
Magnets & 5 & - \\
Magnet power supplies & 126 & 16 \\
Interlocks & 33 & 4 \\
Ion sources and Cockcroft-Walton & 171 & 21 \\
$\quad$ high-voltage supplies & 14 & 2 \\
Cooling water systems & 6 & - \\
Computer control and ciata acquisition & 66 & 8 \\
Production targets & 10 & 1 \\
Pulse-timing systems & 30 & 4 \\
Miscellaneous (utilities, power & & \\
$\quad$ interruptions from lightning, etc.) & - & \\
Total & 796 & \\
\hline \hline
\end{tabular}

The $\mathrm{H}^{-}$source was recycled on an as-needed basis rather than in a (nondowntime-impacting) prescheduled mode. This contributed 76 hours to the downtime figures.

A major vacuum leak at the A-2 target box during cycle 54 was the major beam-off contributor this year.

Beam availability for the WNR/PSR-LANSCE areas was very much impioved compared to that for CY 1988. Significant hardware upgrades were the major contributors to this improvement. 


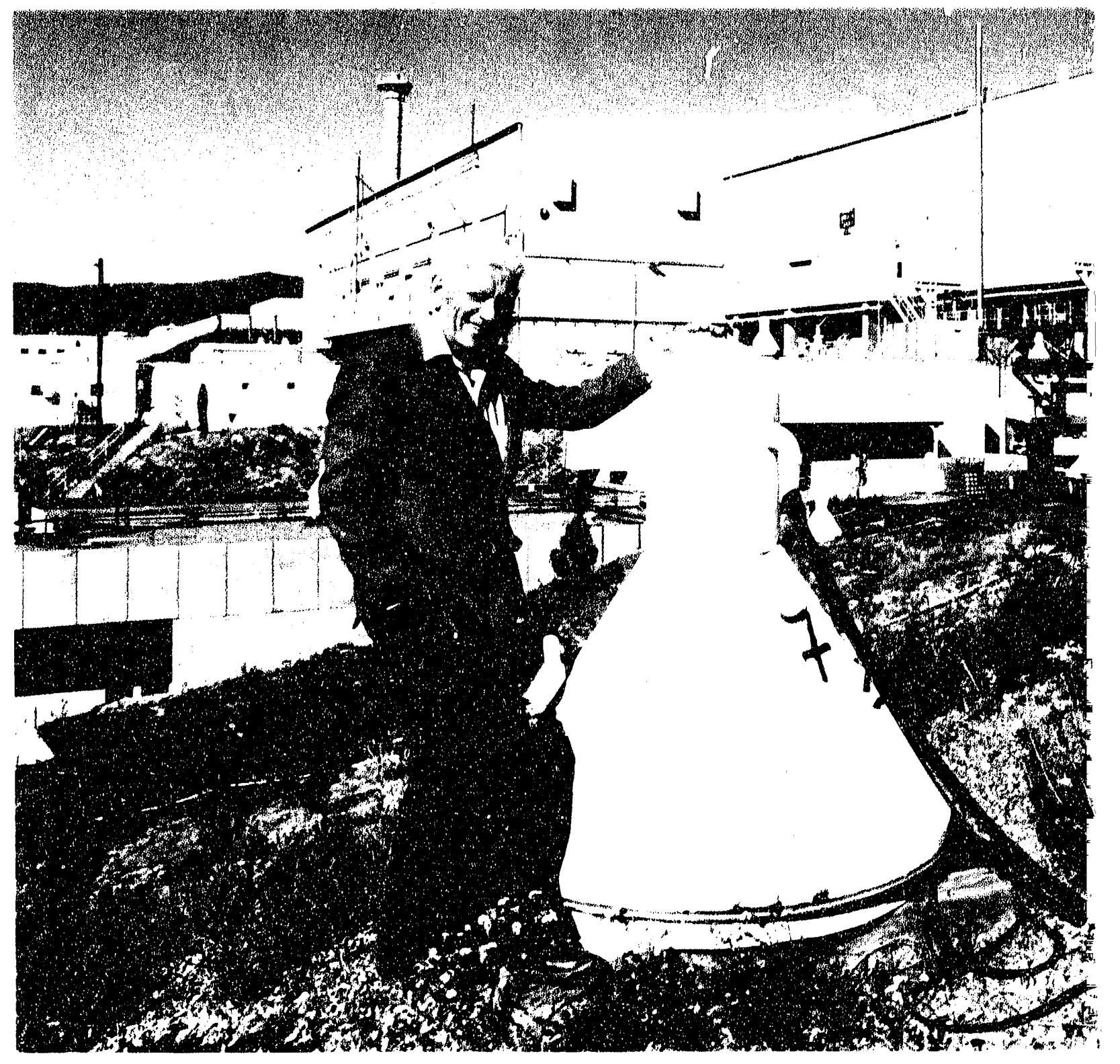




\section{MILESTONES \\ CLINTON P. ANDERSON MESON PHYSICS FACILITY}

1968

Official Ground Breaking

February 15, 1968

Spinoff: Adoption of LAMPF Accelerating Structure

for X-Ray Therapy and Radiography Machines

ca 1968

1970

5-MeV Bcam Achicved

Adoption of a LAMPF Standard Data-Acquisition

System

June 10,1970

August 1970

1971

100-MeV Beam Achicved

June 21, 1971

211-MeV Beam Achicved

August 27, 1971

\section{2}

800-McV Beam Achicved

June 9,1972

Spinoff: First Use of Electrosurgical Forceps in

Open-Heart Surgery (University of New Mexico) September 13, 1972

Discovery of ${ }^{236} \mathrm{Th}$ (Experiment Zero)

September 25, 1972

Dedication to Senator Clinton P. Anderson

Spinoff: First Hyperthermic Treatment of

Animal Tumors

September 29, 1972

October 1972

1973

First $\mathrm{H}^{-}$Injector Bcam

First Sirrultaneous $\mathrm{H}^{+}$and $\mathrm{H}^{-}$Beams

Beam to Area B

First Experiment (\#56) Received Beam

First Meson Production, Beam to Area A
March 28, 1973

May 4, 1973

July 15, 1973

August 24, 1973

August 26, 1973

\section{4}

Beam to Arca A-East

First Medical Radioisotope Shipment

Usable 100- A B Beam to Switchyard

Pi-Mesic Atoms with "Ticklish" Nuclei

First Experimental Pion Radiotherapy

First Tritium Experiment $(80000 \mathrm{Ci})$

Start of Great Shutdown
February 6, 1974

July 30,1974

September 5, 1974

October 13, 1974

October 21, 1974

November 1974

December 24, 1974 
New Precise Measurements of Muonium Hyperfine Structure Interval and $\mu^{+}$Magnetic Moment

1975-77-80

$Q$ Data-Acquisition Software Operational

June 1975

Spinoff: First Use of ${ }^{{ }^{2}} \mathrm{Rb}$ for Myocardial Imaging

in Humans (Donner Lab, Lawrence Berkeley

National Laboratory)

Spinoff: First Hyperthermic Treatment of

Human Cancer (University of New Mexico)

Accelcrator Turnon

Acceptable Simultancous $100-\mu \mathrm{A} \mathrm{H}^{+}$and $3-\mu \mathrm{A} \mathrm{H}^{-}$

Bcams to Switchyard

Production Beam to Arca B

June 1975

July 11,1975

August 1, 1975

September 14, 1975

October 7, 1975

1976

First Pions Through EPICS

Production Beam in Arcas A and A-East:

End of Creat Shutdown

Muon-Spin-Relaxation Program

Spinoff: First Hyperthermic Treatment of

Cancer Eye in Cattle (Jicarilla Reservation)

100- $\mu$ A Production Beam in Area A

Experiment in Atomic Physics $\left(\mathrm{H}^{-}+\right.$laser beam):

Observation of Feshbach and Shape Resonances

in $\mathrm{H}^{-}$

Double Charge Exchange in ${ }^{16} \mathrm{O}$ : LEP Channel

Startup of Isotope Production Facility

HRS Operation Begins

Maintenance by "Monitor" System of

Remote Handling

March 18, 1976

April 5, 1976

June 1976

June 3, 1976

August 1976

October 1976

October 5, 1976

October 15, 1976

November 1976

Fall 1976

1977

Proton Beam to WNR

Polarized-Proton Beam Available

Spinoff: First Practical-Applications Patent

Licensed to Private Industry

Pion Radiotherapy with Curative Intent

Proton-Computed Tomography Program

Experimental Results at Neutrino Facility

Cloud and Surface Muon Beams: SMC

EPICS Operation Begins

300- $\mu$ A Production Beam in Area A
March 12, 1977

April 1977

April 12, 1977

May 1977

June 1977

July 1977

July 1977

August 1977

Fall 1977 
AT Division Established

$\pi^{0}$ Spectrometer Begins Operation

Operation of Polarized-Proton Target

Successful Water-Cooled Graphite Production

Target
January 1, 1978

February 1978

Spring 1978

November 1978

1979

Spinoff: First Thermal Modification of

Human Cornea (University of Oklahoma)

July 11, 1979

600- $\mu$ A Production Bcam in Arca A

November 1979

New Limit on $/ 1-r \gamma$

December 1979

1980

Experimental Measurement of the Strong-Interaction Shift in the 2 $p$ - 1 s Transition for Pionic Hydrogen

Commercial Production of Radioisotopes

1980-81-82

Spin Precessor Begins Operation

Data-Analysis Center Operational

Variable-Energy Opcration

Single-Isobaric-Analog States in Heavy Nuclei

January 1980

February 1980

April 1980

June 1980

June 1980

Spinoff: First Use of ${ }^{2} \mathrm{Rb}$ for Brain Tumor Imaging

in Humans (Donner Lab, Lawrence Berkeley Laboratory)

Production of Fast Muonium in Vacuum

September 1980

Fall 1980

Double-Isobaric-Analog States in Heavy Nuclei

October 1980

Focal-Plane Polarimeter Operational at HRS

Safety Award to LAMPF Users Group, Inc., for

October 1980

Working One Million Man-Hours Since 1975

Without a Disabling Injury

New Measurement of Pion Beta Decay -..-Improved

Test of Conserved-Vector Current

October 27, 1980

November 1980

1981

First Excitation of Ciant Dipole Resonance by

Pion Single Charge Exchange

March 1981

First Observation of Isovector Monopole Resonance

in ${ }^{120} \mathrm{Sn}$ and ${ }^{110} \mathrm{Zr}$ by Pion Single Charge Exchange March 1981

Negative Evidence for Critical Opalescence in ${ }^{41} \mathrm{Ca}$ September 1981 
Average Beam Current of LAMPF Accelerator

Established at $750 \mu \mathrm{A}$

1982

Staging Area Constructed

1982

"Dial-a-Spin" Capability on Line B Permits Different

Spin Orientations for HRS, Line B, and EPB

Simultaneously

1982

Improved Test of Time-Reversal Invariance in

Strong Interactions by Comparison of the

Polarization in the Reaction $p l \rightarrow \vec{n} p p$.

with the Analyzing Power in the

Interaction $n \vec{p}-\mu n$

1982

dt Fusion Catalyzed by Muons

November 1982

1983

LAMPF Accelerator Produces Proton Beam of $1.2 \mathrm{~mA}$

First Observation of $\nu_{e^{-}} e^{-}$Scattering

February 7, 1983

Result for Asymmetry in $\vec{p}$, Scattering Caused by

Parity Violation: $A_{L}=(2.4 \pm 1.1) \times 10^{-7}$

at $800 \mathrm{MeV}$

October 1983

November 1983

1984

Duty Factor $\geq 9 \%$ Achieved

Total Cross Section for $\nu_{e}-e^{-}$Scattering $\sigma_{T}=10^{-44} E_{\nu}(\mathrm{GoV}) \mathrm{cm}^{2}$

February 1984

Clamshell Spectrometer On Line

May 1984

June 1984

High-Intensity $\mathrm{H}^{-}$Source Operational

September 1984

1985

New Beam Stop Installed by Remote-Handling System

High-Intensity $\mathrm{H}^{-}$Injector Operational

Spring 1985

April 1985

New Switchyard Permits Three-Beam Operation

April 1985

Proton Beam to Proton Storage Ring (PSR)

May 1985

Routine Production at Beam Current of $1 \mathrm{~mA}$

Summer 1985

Precise Near-Threshold Measurements of $r^{-} p \rightarrow \pi^{0} n$ Reaction

$\eta$-Meson Production on Nuclei Observed Near

Threshold

July 1985

August 1985 
17-mA Peak Current Achieved

Precise Test of the Relativistic Doppler Effect at

$0.84 c$ by Collision of an Ator \& Beam with

Laser Light
September 1985

1985

1986

Verification of Destructive Interference Between the Charged-Weak and Neutral-Weak Amplitudes in $\nu_{e}$ Scattering (Exp. 225)

Group MP-5 Formed to Maintain and Develop Line D and the Proton Storage Ring 1986

Masses of $12 \mathrm{New}$ Isotopes Measured by TOFI System

1986

Polarized Beam Delivered with $10-\mathrm{MHz}$

Micropulse Structure for Neutron Timing

1986

1987

Neutron Time-of-Flight Facility Short-Flight-Path

Commissioned (New Beam Line in NPL) 1987

Linac Rebuncher Scheme Implemented to Produce

Time-Focused Micropulses at WNR and NTOF

1987

113-MeV Beam Delivered to WNR/Lowest Energy

Delivered by LAMPF to an Experimental Area

Operation with Three Beam Energies to Line A, Line $D$, and Linc $X$

Development of Medium-Resolution (0.2\%) Tune for $P^{3}$

1987

1988

Polarized Nuclear Target Used in Research

$\left({ }^{13} \mathrm{C}\right.$ on HRS for Exp. 955)

1988

CYGNUS Experiment (Uses LAMPF Neutrino

Detectors) Publishes Resulis on Muon

Excess from Ultra-High-Energy Cosmic

Ray Showers

1988

1989

Pion Scattering from Polarized ${ }^{13} \mathrm{C}$

Commissioned MRS Achieving 1.3-MeV Resolution at $800 \mathrm{MeV}$

May 1989

July 1989 
Quantitative Verification of Standard Model

Through Neutrino Electron Scattering

(Exp. 225)

1989

Large Role of Two-Nucleon Correlations

Demonstrated for DCX Reactions on $\mathrm{Ca}$

Isotopes

1989

Limits Established from $\gamma_{\mu} \rightarrow \gamma_{\epsilon}$ Oscillations (Exp. 645)

1989

First Polarized Beam to Experimental Area from OPPIS

1989

Completion of Mcasurement of All $I=1$

Amplitudes in $(1-p)$ Scattering up to $800 \mathrm{MeV}$

1989 


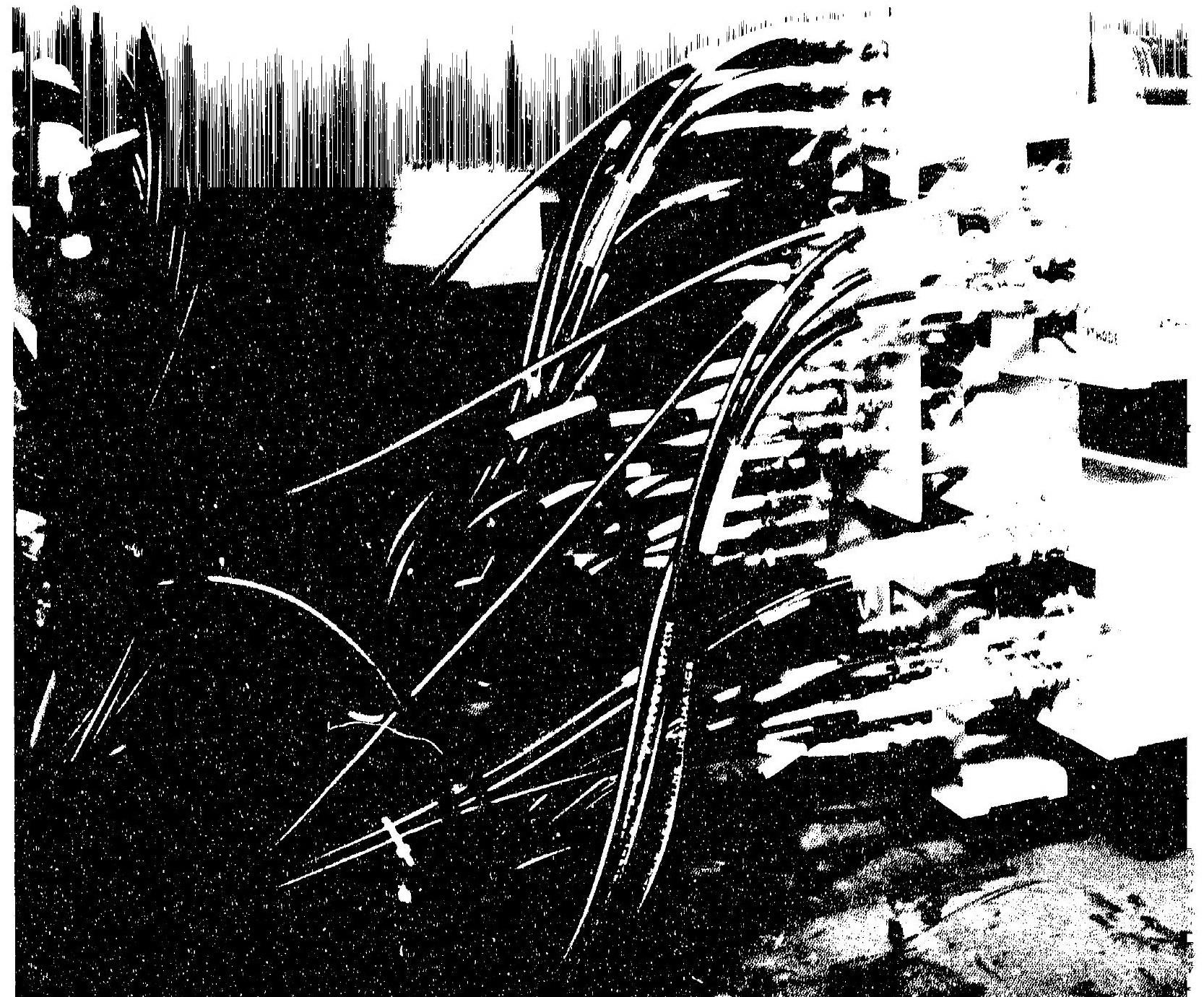




\section{APPENDIX A: Experiments Run in 1989}

\begin{tabular}{llrl}
$\begin{array}{l}\text { Exp. } \\
\text { No. }\end{array}$ & Channel & $\begin{array}{c}\text { Beam } \\
\text { Hours }\end{array}$ & Title \\
\hline 267 & ISORAD & 1858 & $\begin{array}{l}\text { Preparation of Radicisotopes for Medicine and } \\
\text { the Physical Sciences Using the LAMPF Isotope } \\
\text { Production Facility }\end{array}$
\end{tabular}

645 Neutrino-A 1858 A Search for Neutrino Oscillations at LAMPF

$957 \quad P^{3}$

310 Inclusive Pion Double Charge Exchange in Light $p$-Shell Nuclei

969 SMC 679 MEGA - Search for the Rare Decay $\mu^{+} \rightarrow e^{+} \gamma$

$978 P^{3} \quad 90$ A Coincidence Measurement of Pion Double Charge Exchange: ${ }^{4} \mathrm{He}\left(\pi^{+}, \pi^{-} p\right)^{3} p$.

1023 LEP

1025 LEP

$1026 P^{3}$

1040 NTOF

1054 TTA

1064 EPICS

1073 SMC

1081 EPICS

1098 LEP

1100 TTA
925 Analyzing Power Measurements for the $\left(\pi^{+}, \pi^{0}\right)$ Reaction on a Polarized ${ }^{13} \mathrm{C}$ Target

613 Pion Elastic and Inelastic Scattering from Polarized ${ }^{13} \mathrm{C}$

190 A Study of the ${ }^{3} \mathrm{H}\left(\pi^{+}, \pi^{0}\right)^{3} \mathrm{He}$ Reaction

14 Spin Variable Measurements for the $\left(\vec{p}, n^{\prime}\right)$ Inclusive Reaction on Several Nuclei

634 Ultra High-Precision Measurements on Muonium Ground State: Hyperfine Structure and Muon Magnetic Moment

813 Pion Scattering from ${ }^{3} \mathrm{H}$ and ${ }^{3} \mathrm{He}$ Near $180^{\circ}$ in the Region of the $\Delta_{1232}$ Resonance

363 Measurement of Muonium to Antimuonium Conversion with Improved Sensitivity

173 Double Charge Exchange Within the $\left(f_{7 / 2}\right)^{n}$ Shell-Model Space

271 Energy Dependence of Low-Energy Double Charge Exchange

393 Mass Measurements of Neutron-Rich Nuclei with $Z=18 \cdots 32$ 


\begin{tabular}{|c|c|c|c|}
\hline $\begin{array}{l}\text { Exp. } \\
\text { No. }\end{array}$ & Channel & $\begin{array}{l}\text { Beam } \\
\text { Hours }\end{array}$ & Title \\
\hline 1103 & $\mathrm{p}^{3}$ & 276 & $\begin{array}{l}\text { Measurement of the Ratio of }\left(\pi^{+}, \pi^{+^{\prime}}\right) \text { vs } \\
\left(\pi^{-}, \pi^{-}\right) \text {at } T_{\pi}=450 \mathrm{MeV} \text { on }{ }^{13} \mathrm{C},{ }^{14} \mathrm{C}, \\
\text { and }{ }^{15} \mathrm{~N}\end{array}$ \\
\hline 1104 & $P^{3}$ & 266 & $\begin{array}{l}\text { Study of the Giant Quadrupole Resonance by } \\
\text { High-Energy Pion Scattering }\end{array}$ \\
\hline 1107 & $p^{3}$ & 138 & $\begin{array}{l}\text { Studies of Pion Double Charge Exchange } \\
\text { Scattering at Energies Above the } \Delta \\
\text { Resonance }\end{array}$ \\
\hline 1115 & SMC & 160 & $\begin{array}{l}\text { Characterization of High-Temperature Super- } \\
\text { conductors by Muon Spin Relaxation }\end{array}$ \\
\hline 1118 & EPICS & 799 & $\begin{array}{l}\text { Isospin Splitting of Isovector Resonances in Pion } \\
\text { Double Charge Exchange }\end{array}$ \\
\hline 1121 & HIRAB & 211 & $\begin{array}{l}\text { High Excitations and Double Escape in the } \\
\text { Negative Hydrogen Ion }\end{array}$ \\
\hline 1123 & NTOF & 254 & $\begin{array}{l}\text { Measurement of Gamow-Teller Strength in the } \\
{ }^{16} \mathrm{O}(p, n){ }^{16} \mathrm{~F} \text { Reaction }\end{array}$ \\
\hline 1127 & HIRAB & 297 & $\begin{array}{l}\text { Multiphoton Detachment of Electrons from the } \\
\mathrm{H}^{-} \text {Ion }\end{array}$ \\
\hline 1135 & HRS & 52 & $\begin{array}{l}\text { Feasibility Study of Tagged Eta Meson Produc- } \\
\text { tion in } p+{ }^{3} \mathrm{H} \rightarrow{ }^{4} \mathrm{He}+\eta\end{array}$ \\
\hline 1139 & HRS & 283 & $\begin{array}{l}\text { Testing of Radiation Resistance of Read-Out } \\
\text { Chips for Use in High Rate Nuclear and Particle } \\
\text { Physics Applications }\end{array}$ \\
\hline 1151 & SMC & 380 & $\begin{array}{l}\text { Experimental Investigation of Muon Catalyzed } \\
\text { Fusion }\end{array}$ \\
\hline 1153 & $P^{3}$ & 673 & $\begin{array}{l}\text { Quasifree Pion Charge Exchange Above the } \\
\text { Delta }\end{array}$ \\
\hline 1154 & EPICS & 139 & $\begin{array}{l}\text { Properties of the Giant Dipole Resonance Built } \\
\text { on the Isobaric Analog State }\end{array}$ \\
\hline 1160 & EPICS & 234 & $\begin{array}{l}\text { Excitation Function of DIAS Transitions in the } \\
\text { Nickel Isotopes }\end{array}$ \\
\hline
\end{tabular}




\section{APPENDIX A}

Experiments Run in 1989

\begin{tabular}{|c|c|c|c|}
\hline $\begin{array}{l}\text { Exp. } \\
\text { No. }\end{array}$ & Channel & $\begin{array}{l}\text { Beam } \\
\text { Hours }\end{array}$ & Title \\
\hline 1161 & EPICS & 279 & Double Charge Exchange on Se Isotopes \\
\hline 1162 & $P^{3}$ & 211 & Excitation Functions of Double Resonances \\
\hline 1164 & SMC & 38 & $\begin{array}{l}\text { Measurement of the Polarization of Negative } \\
\text { Muons Stopped in Polarized Noble Gases }\end{array}$ \\
\hline 1165 & REF & 925 & SINQ-Target Entry-Window Test in Los Alamos \\
\hline 1183 & $\begin{array}{l}\text { Nuclear } \\
\text { Chemistry } \\
\text { Lab }\end{array}$ & 4 & Proton Irradiations of ${ }^{92} \mathrm{Mo}$ and nat Mo Foils \\
\hline
\end{tabular}




\section{APPENDIX B: New Proposals During 1989}

Exp.

No.

Spokesperson

1136 R. J. Peterson Univ. of Colorado

1137 T. N. Taddeucci Los Alamos

1138 B. L. Clausen Univ. of Virginia

R. A. Lindgren Univ. of Virginia

1139 C. M. Hoffman Los Alamos

H. F. W. Sadrozinski UC Santa Cruz

W. F. Sommer Los Alamos

1140 C. F. Moore Univ. of Texas, Austin

C. L. Morris Los Alamos

1141 E. Gülmez UCLA

1142 F. T. Baker Unuv. of Georgia

K. W. Jones Los Alamos

1143 C. L. Morris Los Alamos

C. S. Mishra Los Alamos

R. D. Ransome Rutgers Univ.

1144 B. J. Lieb George Mason Univ.

L.-C. Liu Los Alamos
Title

Pion Double Charge Exchange to High Excitations in Complex Nuclei

Measurement of $D_{L L}\left(0^{\circ}\right)$ for $1^{+}(p, n)$

Transitions in $p$-Shell Nuclei

Pion Scattering to $6^{-}$Stretched States in ${ }^{32} \mathrm{~S}$

Testing of Radiation Resistance of Read-Out Chips for Use in High-Rate Nuclear and Particle Physics Applications

Search for the $\eta^{0}$ Component in $\pi$-DCX

A Systematic Study of $(p, n)$ Cross Sections on Moderate Mass Nuclei at LAMPF Energies

Isovector/Isoscalar Decomposition for the $\Delta S=1$ Continuum in ${ }^{40} \mathrm{Ca}$

Study of Pion Absorption in ${ }^{6} \mathrm{Li},{ }^{7} \mathrm{Li},{ }^{\prime} \mathrm{Be}$, ${ }^{10} \mathrm{~B}$, and ${ }^{11} \mathrm{~B}$

Studies of Eta-Nucleus Bound Systems 
Exp.

No. Spokesperson

1145 X.-Y. Chen Univ. of Colorado

1146 J. Rapaport Ohio Univ.

J. L. Ullmann

Los Alamos

R. L. Boudrie

Los Alamos

1147 J. C. Hicbert

Texas A\&M Univ.

L. C. Northcliffe

Texas A\&M Univ.

S. Nath

Texas A\&M Univ.

1148 M. K. Jones

Univ. of New Mexico

S. H. Yoo

Univ. of Texas, Austin

1149 A. Fazely Louisiana State Univ.

H. T. Fortune

Univ. of Pennsylvania

L.-C. Liu

Los Alamos

1150 C. F. Moore Univ. of Texas, Austin

S. Mordechai Ben-Gurion Univ.

1151 M. Leon

Los Alamos

M. A. Paciotti

Los Alamos

S. E. Jones

Brigham Young Univ.

1152 J. N. Knudson Los Alar.ios
Title

Measurements of Complete Spin

Observables in the Quasifree Region

Quasielastic and Quasifrec Pion Production via the $(n, p)$ Reaction at $800 \mathrm{McV}$

Test of Charge-Symmetry-Breaking in the Reaction $n p \rightarrow d \pi^{0}$

$\left(\pi, \pi^{\prime} p\right)$ Coincidence Measurement Above the $\Delta_{3,3}$ Resonance

Study of the $0^{+} \rightarrow 0^{+}$(g.s.) Transition with the Low-Energy Pion Double Charge Exchange Reaction on ${ }^{128} \mathrm{Te}$ and ${ }^{130} \mathrm{Te}$

Search for Double Giant Dipole Excitations Using $\left(\pi^{-}, \pi^{+}\right)$Pion Double Charge

Exchange

Experimental Investigation of MuonCatalyzed Fusion

Angular Distributions of Nonanalog States in ${ }^{14} \mathrm{C}$ 
Exp.

No. Spokesperson

1153 R. J. Peterson Univ. of Colorado

S. Hфibråten Univ. of Colorado

1154 H. T. Fortune Univ. of Pennsylvania

K. W. Johnson Univ. of Texas, Austin

S. Mordechai Ben-Gurion Univ.

1155 C. Pillai UCLA

K. S. Dhuga Grorge Washington Univ.

W. J. Briscoe George Washington Univ.

1156 B. H. King Stanford Univ.

D. Počanić Univ. of Virginia

1157 J. M. O'Donnell Univ. of Pennsylvania

1158 K. K. Seth Northwestern Univ.

1159 K. K. Seth Northwestern Univ.

1160 J. D. Silk Univ. of Pennsylvania

1161 A. Fazcly Louisiana State Univ. H. T. Fortune Univ. of Pennsylvania S. Mordechai Ben-Gurion Univ.
Title

Quasifree Pion Charge Exchange Above the Delta

Properties of the Giant Dipole Resonance Built on the Isobaric Analog State

Elastic Pion Scattering from ${ }^{3} \mathrm{H}$ and ${ }^{3} \mathrm{He}$ in the Region of the $\Delta$ (1232) Resonance

Study of the Isospin Structure and Charged Particle Decay of the Giant Dipole Resonance Excited by Pion Single Charge Exchange in Mass 9 Nuclei

DIAS Q Value and Cross Section Systematics for $A>58$

A Measurement of the Width of the Double Analog State in ${ }^{8} 8 \mathrm{Zr}$

A Study of the ${ }^{42} \mathrm{Ca}\left(\pi^{+}, p\right)^{41} \mathrm{Ca}$ Reaction

Excitation Function of DIAS Transitions in the Nickel Isotopes

Double Charge Exchange on Se Isotopes 
Exp.

No. Spokesperson

1162 J. D. Silk

Univ. of Pennsylvania

1163 K. K. Seth

Northwestern Univ.

1164 G. D. Cates

Princeton Univ.

P. A. Souder Syracuse Univ.

1165 G. Bauer PSI

1166 D. S. Oaklcy Univ. of Colorado

R. J. Peterson Univ, of Colorado

1167 C. F. Moore Univ. of Texas, Austin

R. J. Peterson Univ. of Colorado

1168 B. S. Flanders American Univ.

P. G. Roos Univ. of Maryland

1169 T. Kobayashi KEK

1170 C. F. Moore Univ of Texas, Austin

S. Mordechai Ben-Gurion Univ.

1171 C. F. Moore Univ. of Texas, Austin

S. Mordechai Ben-Gurion Univ.
Title

Excitation Functions of Double Resonances

A New Measurement of the Chiral Symmetry-Breaking Paraineter

Measurement of the Polarization of Negative Muons Stopped in Polarized Noble Gases

SINQ-Target Entry-Window Test in Los Alamos

Pion Scattering to $6^{-}$States of ${ }^{26} \mathrm{Mg}$

Pion Single Charge Exchange as Pathways for Double Charge Exchange

Study of Pion Production on Light Nuclei With Intermediate Energy Protons

Search for a Soft Giant Dipole Resonance in ${ }^{11}$ Li Nuclei via the Pion-Induced Double Charge Exchange Reaction

Scarch for Double Gamow-Teller Excitations Using Pion Double Charge Exchange

Isospin Splitting of Isovector Resonances in Pion Single Charge Exchange 
Exp.

No. Spokesperson

1172 A. Klein

New Mexico State Univ.

N. S. Chant

Univ. of Maryland

1173 X.-Q. Lu UC Irvine

1174 C. L. Morris Los Alamos

J. D. Zumbro

MIT

R. J. Peterson

Univ, of Colorado

1175 D. K. Dehnhard Univ. of New Mexico

G. R. Burleson

New Mexico State Univ.

1176 J. M. O'Donnell Univ. of Pennsylvania

1177 E. R. Kinney Argonne

J. L. Matthews

MIT

1178 G. R. Burleson New Mexico State Univ. J. R. Comfort Arizona State Univ.

1179 D. Počanić Univ. of Virginia

1180 A. B. Kurepin Inst. of Nuclear Research

C. L. Morris Los Alamos
Title

Cross Sections and Analyzing-Power

Measurements for ${ }^{7} \mathrm{Li}(\pi, \pi p)^{6} \mathrm{He}$ with

Good Kinematic Resolution

A Proposal to Search for $\bar{\nu}_{\mu}-\bar{\nu}_{e}$ Oscillations with High Sensitivity at LAMPF

Quasi-Elastic and Continuum Pion Scattering Above $\Delta_{3 / 2,3 / 2}(1232 \mathrm{MeV})$ Energies

Analyzing Power Measurements for the $\left(\pi^{+}, \pi^{0}\right)$ Reaction on a Polarized ${ }^{13} \mathrm{C}$ Target

DIAS Q Value Measurements for $A>58$

Inclusive Pion Single Charge Exchange in ${ }^{3} \mathrm{He}$ and ${ }^{4} \mathrm{He}$

Polarization Asymmetry Measurements for the ${ }^{1} \mathrm{H}\left(\pi^{-}, \pi^{0}\right) n$ Reaction Between 45 and $190 \mathrm{MeV}$

Reaction $\pi^{+} p \rightarrow \pi^{+} \pi^{0} p$ Near Threshold

Investigation of Narrow $\Delta \Delta$ and $\Delta$-Isobaric States in Nuclei 
Exp.

No. Spokesperson

1181 D. Počanić Univ. of Virginia

S. S. Hanna Stanford Univ.

1182 A. H. Husscin King Fahd Univ. of Petrolcum and Minerals

1183 D. C. Moody Los Alamos

1184 E. R. Sugarbaker Ohio State Univ.

T. N. Taddeucci Los Alamos

\section{Title}

Isospin Structure of the Isovector Giant Dipole Resonance in Mass 9 Nuclei Excited by Pion Single Charge Exchange

Measuring Neutron-Neutron Scattering Length and Effective Range Using the

${ }^{2} \mathrm{H}\left(\pi^{-}, 2 n\right) \gamma$ Reaction

Proton Irradiations of ${ }^{92} \mathrm{Mo}$ and nat Mo Foils Measurement of Analyzing Powers for the ${ }^{14} \mathrm{C}(p, n)$ (IAS) Transition 


\section{APPENDIX C: LAMPF Visitors During 1989}

\author{
Alexander Abashian, Virglnia Polytechnic Instllute and State Univ., USA \\ David Adams, Rice Univ., USA \\ Terry Adams, Texas Tech Unlv., USA \\ Hyo Ahn, Yale Univi, USA \\ Richard Alarcon, Arizona State Univ., USA \\ Dimitris Alexandreas, Univ. of Callfornla, Irvine, USA \\ Thomas Altman, Oswego High School, Oswego, New York; USA \\ Pierte Amaudruz, TRIUMP, Canada \\ Alfonso Anaya, National Nuclear Research Institute, Mexico \\ Hans Anders, CERN, Switzerland \\ Alan Anderson, Nonafrillated, USA \\ Matthew Anderson, Ohlo State Univ., USA \\ Konrad Aniol, California State Univ., Los Angeles, USA \\ Masaki Arima, Univ. of Tokyo, Japan \\ Jyunichi Asaba, Mitsubishl Heavy Industries, Iric., Japan \\ John Ashley, Obcrilln College, USA \\ Kenneth Ashley, liast Texas State Univ., USA \\ Naftali Auerbach, 'Tel-Aviv Univ., Israel \\ Jorthrn Aukdal, Riso National Laboratory, Denmark \\ Richard Baartman, TRIUMF, Canada \\ Mark Bachman, Unlv. of Texas, USA \\ Andreas Badertscher, ISI, Switzerland \\ F. Baker, Univ, of Cerorgia, USA \\ Martin Barlett, Unlv. of Texas, USA \\ David Barlow, Los Alamos National Laboratory, USA \\ Michael Barnes, Los Alamos National Laboratory, USA \\ Bruce Barnett, Univ, of Tokyo, Japan \\ Laurence Barrin, Cl:RN, Switwerland \\ Robin Barrone, Univ, of California, Irvine, USA \\ Johann Bartel, CRN/PNTL, France \\ Bernd Bassalleck, Univ, of New Mexico, USA \\ Gunter Bauer, ISII Switzerland \\ David Beatty, Rulyers Univ., USA \\ Michael Beddo, New Mexico State Univ., USA \\ Alexander Belov, Instltute for Nuclear Research, USSR \\ Donald Benton, I'rinceton Univ., USA \\ David Berley, Univ. of Maryland, USA \\ Barry Berman, George Washington Univ., USA \\ Brian Berman, New Mexico State Univ., USA \\ Jonathan Berman, Ceorge Washington Univ, USA \\ Hans Bethe, Corncll Univ., USA \\ Steven Biller, Univ, of California, Irvine, USA \\ Louis Bimbot, IIN, Orsay, France \\ Ewart Blackmore, TRIUMF, Canada \\ Marek Bleszynski, UCIA, USA \\ Werner Bloeglin, MIr, USA \\ Carolus Boekema, San Jose State Undv., USA \\ Edmund Boschitz, Institul fur Kernphysik, W. Germany \\ Franco Bradamantc, INFN, Trieste, Italy \\ Michael Braunstein, Univ, of Colorado, USA \\ Herbert Breuer, Univ. of Maryland, USA \\ Bernhard Brinkmoller, Institut fur Kernphyslk, W. Cermany
}




\section{APPENDIX C}

LAMPF Visitors During 1989

William Briscoe, George Washington Univ, USA

Melynda Brooks, Univ, of New Mexico, USA

Geoffrey Brown, Abllene Christian Univ., USA

Johannes Bruckner, Max-Planck-Institut fur Chemie, W. Cermany

Michael Bryan, Univ. of Texas, USA

Howard Bryant, Univ, of New Mexico, USA

Ray Burge, TRIUMF, Canada

Michael Burlein, Univ, of Pennsylvania, USA

George Burleson, New Mexico Slate Univ., USA

Alan Burns, CERN, Switzerland

Mary Burns, Los Alamos National Laboratory, USA

Peter Busch, Michigan State Univ., USA

Pavel Bystricky, Ecole Nationale Superieure d'Electrochlule, France

Robert Cady, Univ. of Notre Dame, USA

Augustine Caffrey, BC\&G, Idaho, Inc., USA

John Cameron, Indiana Univ., USA

David Cardon, Brigham Young Univ., USA

Rebecca Caress, Ceorge Washington Univ., USA

Daniel Carman, Nonaffiliated, USA

Hamilton Carter, Ohio State Univ., USA

Gordon Cates, Princeton Univ., USA

Chung-Yun Chang, Univ. of Maryland, USA

Walter Chapman, Texas A\&M Univ., USA

Xiao-Yan Chen, Univ, of Colorado, USA

Francis Chmely, Nonafilliated, USA

Douglas Ciskowski, Yale Univ., USA

Nelson Claytor, Univ. of Pennsylvania, USA

Francis Close, Univ, of Tennessee, USA

Steven Coberly, Brown Univ., USA

Stanley Cohen, Los Alamos National Laboratory, USA

Joseph Comfort, Arizona State Univ., USA

Hope Concannon, Valparaiso Univ., USA

Peter Cooper, Fermilab, USA

Dylan Cors, MIT, USA

Michael Craddock, 'l'RIUMF, Canada

Kevin Cranston, New Mexico State Univ., USA

Robert Crittenden, Univ, of Pennsylivania, USA

John Crocker, Univ, of Chicago, USA

Jack Crow, T'emple Univ., USA

Guy Danner, Princeton Univ., USA

Norman Davison, Univ. of Manitoba, Canada

W. Dawson, TRIUMi:, Canada

Wayne Dawson, San Jose State Univ., USA

James DeKorse, Arizona State Univ., USA

R. DeLay, Univ, of Callfornia, Irvine, USA

Dietrich Dehnhard, Univ. of Minnesota, USA

Steven Delucia, Ohio State Univ., USA

Michael Devereux, Vartham College, USA

Kalvir Dhuga, Ceorge Washington Univ., USA

Eduardo Diaz, California State Univ., L.os Angeles, USA

Brenda Dingus, NASSA-Goddard, USA

Gerard Dion, Univ. of Callfornia, Irvine, USA 
Donald Dohan, TRIUMF, Canada

Alan Donley, Abilene Christian Univ., USA

Ed Doran, Univ. of Texas, USA

Marla Dowell, MIT, USA

Sinisa Dragic, George Washington Univ., USA

Martin Dubs, ISI, Switzerland

Timothy Duty, Virginin Polytechnic Institute and State Univ., USA

Mario Dzemidzic, Univ. of Houston, USA

Morton Eckhause, College of William \& Mary, USA

Juan Elizando, Univ, of New Mexico, USA

Robert Ellsworth, George Mason Univ., USA

Roy Emery, PSI, Switzorland

Terry Enegren, T'RIUMF, Canada

Daniel Enger, Los Alamos National Laboratory, USA

Peter Englert, San Jose State Univ., USA

Torleif Ericson, CERN, Switzerland

David Ernst, Texas A\&M Univ, USA

Ali Fazely, Lousiana State Univ., USA

Gwennaele Fichant, Univ. Claude Bernard-Lyon I, France

David Fink, Univ. of Pennsyli ania, USA

Bruce Flanders, American Univ., USA

Jeffrey Flint, Sar: Jose State Univ., USA

Wilson Fong, MIT, USA

H. Fortune, Univ. of Denusylvania, USA

Christen Frankle, North Carolina State Univ., USA

Barbara Franklin, Arizona State Univ., USA

Stuart Freedman, Argorne, USA

Eernard Frois, CTEN-Saclay, France

Takamitsu Fugiu, Nikon Corp., Japan

Brian Fujikawa, A.rgonne, USA

Kenji Fukushima, 'lokyo Metropolitan Univ., Japan

Carl Gagliardi, Texas A\&M Univ., USA

Frank Gallagher, Univ. of Texas, USA

Robert Garnett, Lo Namos National Laboratory, USA

Thomas Gaussiran II, Rice Univ., USA

Didier Gavillet, PSI, Switzerland

M. Gazzaly, Nonaffiliated, USA

Lisa Gill, Texas A\&M Univ., USA

Ronald Gilman, Rutgers Univ., USA

Paolo Giubellino, Univ. of Torino, Italy

Charles Glashausser, Rutgers Univ., USA

George Glass, Texas A\&M Univ., USA

Robert Glasser, Los Nlamos National Labe . y, USA

Bruce Gluckman, Univ. of Pennsylvania, USA

Johann Georgen, Arizona State Univ., USA

Charles Goodman, Indiana Univ., USA

Jordan Goodman, Univ. of Maryland, USA

Christopher Gould, North Carolina State Univ., USA

Andrew Green, Rutigers Univ., USA

Keith Griffioen, Univ. of Pennsyivania, USA

Fedor Guber, Instituic for Nuclear Research, USSR

Gilbert Guignard, Cl:RN, Switzerland 
Erhan Gulmez, UCLA, USA

David Haase, North Carolina State Univ., USA

Ernst-Ulrich Hacbel, CERN, Switzerland

Quamrul Haider, Fordham Univ., USA

Todd Haines, Univ, of Maryland, USA

Jurgen Halbritter, Karnforschungszentrum, W. Coermany

Aksel Hallin, Princeton Univ., USA

Francis Halzen, Univ. of Wisconsin, USA

Jeffrey Harris, Brigham Young Univ., USA

Philip Harris, Univ. of New Mexico, USA

Ferenc Hegedus, IXsl, Switzerland

Walter Hensley, Battelle Pacific Northwest laboratory, USA

Dierk Herlach, PSI, Switzerland

Rogelio Hernan dez, National Nuclear Research Institute, Mexico

John Hiebert, Texas A\&M Univ., USA

Norton Hintz, Univ. of Minnesota, USA

Gerald Hoffmann, Univ. of Texas, USA

Steinar Hoibraten, Univ. of Colorado, USA

Karl Holinde, Univ, of Bonn, W. Germany

Richard Holmes, Syracuse Univ., USA

Elliott Horch, Stanfurci Univ., USA

Weidong Huang Indiana Univ., USA

E. Hughes, Stanford Univ., USA

Vernon Hughes, Yale Univ., USA

Ed Hungerford, Univ. of Houston, USA

Nadeem Hussain, Stanford Univ., USA

George Igo, UCLA, USA

Richard Imlay, Louisiana State Univ., USA

C. H. Ingram, I'SI, switzerland

T. Inoue, Japan Atomic Energy Research Institute, Japan

Erik Insko, Univ. of Pennsylvania, USA

Larry Isenhower, Abilene Christian Univ., USA

Ryozo Ito, Nikon Corp., Japan

Rex Ivie, Univ. of P'ennsylvania, USA

Muhammad Jahan, Memphis State Univ., USA

Mark Jakobson, Univ. of Montana, USA

Randolph Jeppesen, Univ. of Montana, USA

Keven Johnson, Univ. of Texas, USA

Richard Johnson, 'TRIUMF, Canada

Mark Jones, Rutgers Univ., USA

Steven Jones, Brigham Young Univ., USA

David Jordan, MIT', USA

Charles Jui, Stanford Univ., USA

Klaus Jungmann, Physikalischer Institut de Universitat, $W$. Cermany

Zenzaburo Kabcya, Mitsubishi Heavy Industries, Inc., Japan

Marios Kagarlis, Univ. of Pennsylvania, USA

George Kahrimanis Univ. of Texas, USA

Andrew Kaluzniacki, Univ. of California, Santa Cruz, LSA

John Kane, College of William \& Mary, USA

Ju Hwan Kang Univ. of California, Riverside, USA

Theo Kas apiperis, Uiniv, of Eriangen-ivurniverg, $\dddot{v}$. Germany

Kelly Kawan, UClA, USA 


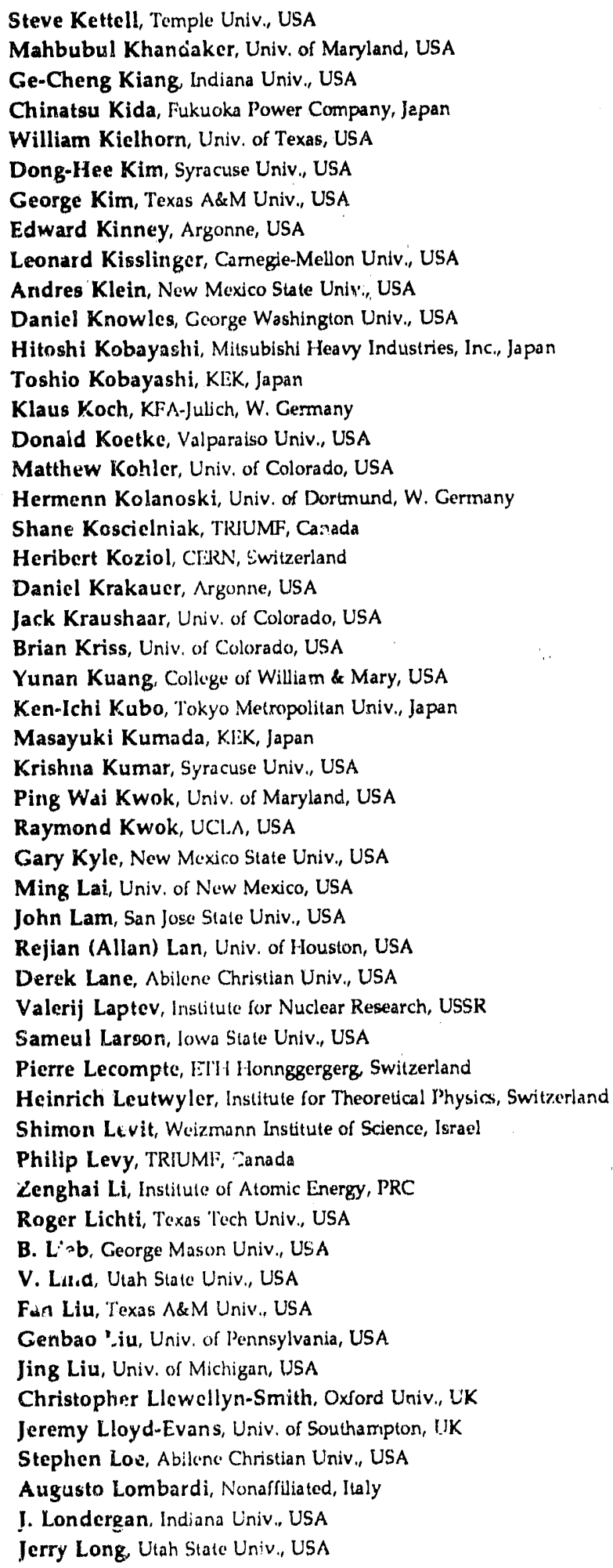


David Lopiano, Argonne, USA

Haolin Lu, Instilute of Atomic Energy, PRC

Xing "Qi Lu, Univ. of California, Irvine, USA

Daniel Macek, Univ. of California, Berkeley, USA

Anthony Mack, Univ. of Minnesota, USA

George MacKenzic,TRIUMF, Canada

Edward MacKerrow, Univ. of New Mexico, USA

John Maddox, MacMillan Journals Ltd, UK

Alfred Mann, Univ. of Pennsylvania, USA

Robert Manweiler, Valparaiso Univ., USA

Donald Marchlenski, Ohio State Univ., USA

Jeremy Margulies, Stanford Linear Accelerator Center (SLAC), USA

John Markey, Yale Univ., USA

William Marterer, Louisiana State Univ., USA

Akira Masaike, Kyoto Univ., Japan

Teiichiro Matsuzaki, Univ, of Tokyo, Japan

Edward Matthews, George Washington Univ., USA

John Matthews, Johns Hopkins Univ., USA

June Matthews, MII', USA

Bjorn Matthias, Yale Univ., USA

James Maxwell, Brigham Young Univ., USA

Bill Mayes, Univ. of Ilouston, USA

James McDonald, Univ. of Texas, USA

Joseph McDonald, Univ. of Texas, USA

Marilyn McDonald, Univ. of Texas, USA

Kenneth McFarlane, Temple Univ., USA

Patrick McGraw, Los Alamos National Laboratory, USA

Thomas Mcllvain, MI'I, USA

Matthew McKinzic, Univ. of Pennsylvania, USA

Kok-Heong McNaughton, Univ. of Texas, USA

David Measday, TRIUMF, Canada

David Mercer, Univ. of Colorado, USA

William Metcalf, Louisiana State Univ., USA

Zein-Eddine Meziani, Stanford Univ., USA

Dimitris Mihailidis, Univ. of Minnesota, USA

William Miles, TRIUMF, Canada

Harry Miley, Ballelle Pacific Northwest Laboratory, USA

William Miller, Univ. of New Mexico, USA

Wyatt Miller, Univ, of Maryland, USA

David Mince, Brigham Young Univ., USA

Vinod Mishra, Ohio State Univ., USA

Gary Mitchell, North Carolina State Univ., USA

Joseph Mitchell, McCill Univ., Canada

Takashi Miyachi, Univ. of Tokyo, Japan

Motoharu Mizumoto, Japan Atomic Energy Research Institute, Japan

Amir Mohagheghi, Univ. of New Mexico, USA

Mehrdad Mohebi, Univ. of New Mexico, USA

Edward Moler, Texas A\&M Univ., USA

C. F. Moore, Univ, of Texas, USA

Shaul Mordechai, Ben-Gurion Univ., Israel

Dane Morgan, Now Mexico State Univ., USA

Sheila Morris, Univ. of T'exas, USA 
Hans Mundinger, lhysikalisches Institut Univ. Heidelberg, W. Germany

Lyle Murphy, Univ, of Colorado, USA

Tomofumi Nagae, Univ. of Tokyo, Japan

Kanetada Nagamine, Univ. of Tokyo, Japan

Donna Naples, Univ, of Maryland, USA

James Napolitano, CLBBAF, USA

Bernard Nefkens, UCLA, USA

James Nelson, Argonne, USA

Nathan Newbury, Princeton Univ., USA

Nancy Nicholas, George Washington Univ., USA

Michacl Nitschke, Lawrence Berkeley Laboratory, USA

John O'Donnell, Univ. of Pennsylvania, USA

David Oakley, Univ. of Colorado, USA

Kazushi Ochi, Mitsubishi Intemational Corp., Japan

Yuji Ohashi, Argonne, USA

Fumio Ohkubo, Mitsubishi Heavy Industries, Inc., Japan

Yoshikazu Okumura, Japan Atomic Energy Research Institute, Japan

Fernando Olguin, National Nuclear Research Institute, Mexico

Chris Oram, TRIUMF, Canada

Herbert Orth, Gesellschafi fur Schwerioueuforschung, W. Germany

Eulogio Oset, Universidad de Valencia, Spain

John Otis, Stanford Univ., USA

Jinsong Ouyang, Univ. of Colorado, USA

Juan Pamplona, Universidad de Valencia, Spain

Valentin Pararnonov, Institute for Nuclear Research, USSR

Hojoon Park, MI'T, USA

Gianni Pauletta, Formilab, USA

Roy Peterson, Univ. of Colorado, USA

Yuri Petrov, Leningrad Nuclear Physics Institute, USSR

Richard Phelps, Univ. of Houston, USA

Eliazer Piasetzky, 'Tel-Aviv Univ., Israel

Leo Piilonen, Virginia Polytechnic Institute and State Univ., USA

Chandrasekhara Pillai, Los Alamos National Laboratory, USA

Lawrence Pinsky, Univ. of Houston, USA

Anatoli Plavko, Polylechnic Institute of Lenirgrad, USSR

Dinko Pocanic, Univ. of Virginia, USA

Roger Poirier, TRIUMl; Canada

Robert Porter, Stanford Univ., USA

Alan Portis, Univ. of California, Berkeley, USA

Bogdan Povh, Max-Planck-Institut fur Kemphysik, W. Germany

Karlton Powell, $A$ bilene Christian Univ., USA

John Price, UCL $\Lambda$, USA

David Prout, Univ. of Colorado, USA

Michael Purcell, Univ. of Texas, USA

Cheng-Rui Qing, Institute of Theoretical Physics, PRC

Ramaswamy Raghavan, AT\&T Bell Laboratories, Murray I Hill, New Jersey, USA

Jack Rapaport, Ohio Univ., USA

Lee Ratliff, Texas 'Tuch Univ., USA

Bill Rawnsley, TRIUMF, Canada

Glen Rebka, Jr., Univ. of Wyoming, USA

Sven Redsun, Princeton Univ., USA

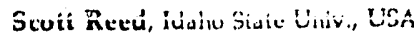


P. Reeder, Reed College, USA

Paul Reeder, Battelle Pacific Northwest Laboratory, USA

Randolph Reeder, Univ, of New Mexico, USA

Stephen Remillard, College of William \& Mary, USA

Peter Riley, Univ. of 'Texas, USA

Daniel Rislov, St. Mary's College, USA

Robert Ristinen, Univ. of Colorado, USA

Barry Ritchie, Arizona State Univ., USA

N. Roberson, Duke Univ., USA

Donald Roberts, Univ. of Michigan, USA

Philip Roos, Univ. of Maryland, USA

Graham Ross, Univ. of Oxford, UK

Thomas Rosson, Univ. of Oklahoma, USA

William Rowe, Univ. of Califomia, Santa Cruz, USA

Michael Sadler, Abilune Christian Univ., USA

Hartmut Sadrozinski, Univ. of California, Santa Cruz, USA

Akiyo Saisho, Fukuoka Power Company, Japan

Kazuko Saisho, Tosu High School, Japan

Glen Sandoval, Lus Namos National Laboratory, USA

Haiduke Sarafian, Pennsylvania State Univ., USA

Kiyokazu Satoh, l'oshiba Company, Keihin Produce, Japan

Kenji Sawada, Sumitomo Heavy Industries, Inc., Japan

H. Schaefer, Yale Univ., USA

Georg Schaffer, KI:K, W. Germany

Erwin Scheidegger, PSI, Switzerland

Friedrick Schlepuetz, $\mid X_{\text {Sl, Switzerland }}$

Helmut Schoenbacher, CliRN, Switzerland

Friedhelm Schreiber, Utah State Univ., USA

George Sechen, MIT, USA

Hardy Seifert, Justus-Liebig-Universitat Giessen, W. Germany

Dennis Sendall, CI:RN, Switzerland

Yurij Senichev, Institute for Nuclear Research, USSR

Seergey Sereshnikov, Institute for Nuclear Research, USSR

Valerij Scrov, Institute for Nuciear Research, USSR

Anil Sethi, Univ. of Minnesota, USA

Gad Shani, Ben-Gurion Univ., Israel

Elena Shaposhnikova, Institute for Nuclear Research, USSR

M. Sharifian, Univ. of New Mexico, USA

Stephen Sharpe, Univ. of Washington, USA

Thomasin Shechan, Los Alamos National Laboratory, USA

Eugene Sherly, Brigham Young Univ., USA

Grant Sheffer, ITIIUMF, Cariada

Tomikazu Shima, Argonne, USA

Ingo Sick, Univ, of Bascl, Switzerland

James Silk, Univ. of Pennsylvania, USA

Anthony Simon, T'exas A\&M Univ., USA

Ivo Slaus, Rudger Buskovic Institute, Yugoslavia

Douglas Smith, Univ, or Pennsylvania, USA

Elton Smith, CEBAl: USA

Gregory Smith, TRIUMF, Canada

Winthrop Smith, Univ. of Connecticul, USA

W. Smythe, Univ. of Colorado, USA 
Michael Snell, USAFA/DFP, USA

Troy Soos, MIT, USA

Vesna Sossi, TRIUMF, Canada

Paul Souder, Syracuse Univ., USA

Harold Spinka, Argonne, USA

Shirvel Stanislaus, Los Alamos National Laboratory, USA

Michael Stark, Univ. of Maryland, USA

William Stephens, Univ. of Virginia, USA

Stephen Sterbenz, Los Alamos National Laboratory, USA

James Stewart, Western Washington Univ., USA

Bruce Storm, Jr., Univ, of Georgia, USA

Evan Sugarbaker, Ohio State Univ., USA

Ivan Supek, Los Alamos National Laboratory, USA

Jeremy Suttom, Oxford Instruments Company, USA

Chen-Yau Tang, Univ. of New Mexico, USA

Liguang Tang Univ. of Houston, USA

Morton Taragin, George Washington Univ., USA

Shuji Tatsumi, Sumitomo Heavy Industries, Inc., Japan

Stuart Taylor, Brigham Young Univ., USA

Vladimir Tiflov, Institute for Nuclear Research, USSR

James Tinsley, Arizona State Univ., USA

W. Tippens, UCLA, USA

Gholam-Husscin Toutounchi-Sarraf, Univ. of New Mexico, USA

Robert Tribble, T'exas A\&M Univ., USA

Gerald Tripard, Washington State Univ, USA

Xiao-Lin Tu, Utah State Univ., USA

Rick Tully, Univ. of Houston, USA

Geoffrey Tyre, Nonafiliated, USA

David Underwood, Argonne, USA

Arkady Vainshtcin, Institute of Nuclear Mhysics, USSR

Leo Van Ausdeln, Texas A\&M Univ., USA

Willen Van Oers, Univ. of Manitoba, Canada

Ludwig Van Rossum, CEN-Saclay, France

James Vasek, Los Nlamos National Laboratory, USA

Manuel Vicente-Vacas, Universidad de Valencia, Spain

Palahalli Vishwanath, Univ. of California, Irvine, USA

Hansdeter Vogel, Interatom, GMBH, W. Germany

Wolfram von Witsch, Rice Univ., USA

Alexei Vorobicv, L.eningrad Nuclear Physics Institute, USSR

Maurizio Vretenar, CFERN, Switzerland

Steven Vrooman, L.cs Namos National Laboratory, USA

Robert Waligurski, Univ. of Illinois, USA

T. Walker, Rutherford-Appleton Laboratory, UK

Mark Wang, MIT, USA

Ming-Jer Wang, Case Western Reserve Univ., USA

Minghong Wang New Mexico State Univ., USA

Yun Wang Indiana Univ., USA

Aaldert Wapstra, National Institute-Nuclear and High Energy Physics, The Nethorlands

Herbert Ward, Univ, of Texas, Austin, USA

Ray Warner, Battelle Pacific Northwest Laboratory, USA

Douglas Watson, Univ. of York, UK

Monroe Wechsler, lowa State Univ., USA 
Zeev Weinfeld, Tel-Aviv Univ., Israel

Lawrence Weinstein, MIT, USA

Ruth Weiss, Tel-Aviv Univ., Israel

Jules Wess, Univ. of Karlsruhe, W. Germany

Charles Whitten, UCLA, USA

Hans-ULrich Wienands, TRIUMF, Canada

Michael Wiescher, Univ. of Notre Dame, USA

Bryan Wildenthal, Univ. of New Mexico, USA

Allen Williams, Univ. of Texas, USA

Donna Williams, Univ. of Texas, USA

Michael Witkowski, College of William \& Mary, USA

David Wolf, Univ. of 'lexas, USA

Kim Woodle, Brookhaven, USA

Dennis Wright, TRIUMF, Canada

S. Wright, Univ, of Chicago, USA

Anna Wyczalkowski, Univ. of Maryland, USA

Chibing Xu, Univ. of New Mexico, USA

Isao Yamane, Kl:K, Japan

Xin-Jua Yang, Peking Univ., PRC

Raymond Yarema, Fermilab, USA

Yi-Fen Yen, Univ, of Minnesota, USA

Sung Yoo, Univ. of 'lexas, USA

Yi-Ju Yu, Univ, of Minnesola, USA

Dahai Zhang, Univ. of Maryland, USA

Weiping Zhang, Los Alamos National Laboratory, USA

Xiao-Gang Zhou, Utah State Univ., USA

Zongyuan Zhou, Nanjing Univ., PRC

Xianzhou Zhu, Duke Univ., USA

Klaus Ziock, Univ. of Virginia, USA

Gisbert zu Putlitz, Physikalisches Institut de Universital, W. Germany

John Zumbro, MIT, USA

Allen Zych, Univ. of California, Riverside, USA 
Information for Contributors
Progress at LAMPF is the progress report of MP Division of Los Alamos National Laboratory. In addition, it includes brief reports on research done at LAMPF by researchers from other institutions and Los Alamos National Laboratory divisions.

Progress at LAMPF is published annually on April 1. This schedule requires that manuscripts be received by December 1 .

Published material is edited to the standards of the Style Manual of the American Institute of Physics. Papers are not refereed, hence presentation in this report does not constitute professional publication of the material nor does it preempt publication in other journals. Readers should recognize that results reported in Progress at LAMPF are sometimes preliminary or tentative and that authors should therefore be consulted in the event that these results are cited.

Contributors can expedite the publication process by giving special care to the following specifics:

1. When possible, furnish computer files for text and MAPPER files for illustrations, together with a hardcopy of your paper. Progress at LAMPF can accept files from computers, stand-alone word processors, and personal computers. TEX files are especially welcome.

2. Drawings and figures submitted should be of quality suitable for direct reproduction after reduction to single-column width, $55 \mathrm{~mm}(2-1 / 4 \mathrm{in}$.). MAPPER files should be printed on laser printers.

3. Figure captions and table headings must be furnished. The A?P Style Manual requires that every figure have a caption that is "complete and intelligible in itself without reference to the text."

4. References must be complete and accurate. If a reference cites a paper submitted for publication, the title of the paper and the journal where it has been submitted or where it is to be published must be given. Laboratory standards prefer that six authors be listed before et al. is used in reference lists.

5. Abbreviations and acronyms should be avoided if possible (in figures and tables as well as text), and when used must be defined.

6. All numerical data should be given in Système International (SI) units.

7. Authors are reminded that it helps the reader to have an introduction that states the purpose(s) of the experiment before presentation of the data.

Research reports should be brief but complete. A list of recent publications relating to the experiment, for separate tabulation in this report, is much appreciated.

Contributors are encouraged to include as authors all participants in experiments so that they may receive credit for authorship and participation.

Questions and suggestions should be directed to Karen Poclakker, Los Alamos National Laboratory, MS H846, Los Alamos, NM 87545. 


\section{Los Alamos National Laboratory is operated by the University of California for the} United States Department of Energy under contract W-7405-ENG.36.

\section{An Affirmatlve Action/Equal Opportunity Employer}

The four previous reports in this series, unclassified, are LA-10738-PR, LA-11()48-PR, LA-11339-PR, and LA-1167()-PR.

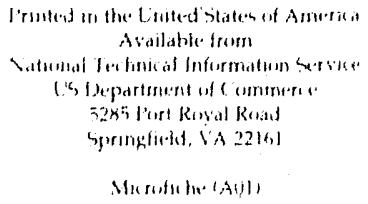

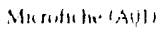

NISS

lage Range Price Code
Nis Page Range: I'rice Conde
Nits

Page Range I'rice Code

\section{Alt}

A15

Al6

A17

A1s

A14 vitls

Irage Range I'rice Code

\begin{tabular}{|c|c|}
\hline $451-475$ & \\
\hline $476=3100$ & \\
\hline $5(1) 1.525$ & \\
\hline $52(-55)$ & \\
\hline 551.575 & \\
\hline $57(6,-6)(1)$ & \\
\hline for13-up & \\
\hline
\end{tabular}

- Contact NTIS for a price quarte. 

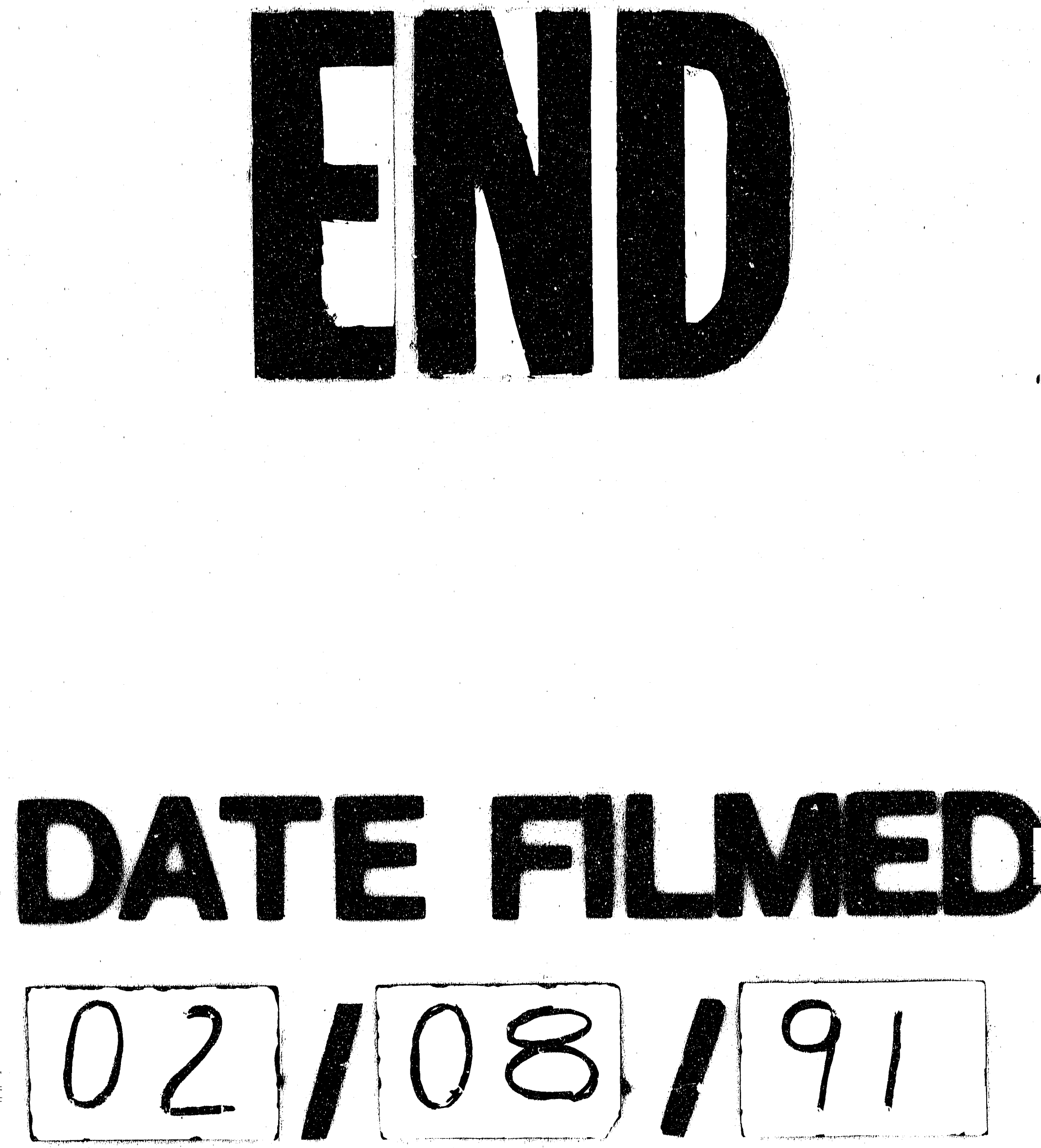


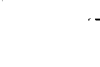

$-$ 